University of Louisville ThinkIR: The University of Louisville's Institutional Repository

Electronic Theses and Dissertations

$4-2015$

\title{
Development of a neuromusculoskeletal computer model in a chondrodystrophic dog.
}

Gregory Russo States

University of Louisville

Follow this and additional works at: https://ir.library.louisville.edu/etd

Part of the Other Biomedical Engineering and Bioengineering Commons

\section{Recommended Citation}

States, Gregory Russo, "Development of a neuromusculoskeletal computer model in a chondrodystrophic dog." (2015). Electronic Theses and Dissertations. Paper 2367.

https://doi.org/10.18297/etd/2367

This Master's Thesis is brought to you for free and open access by ThinkIR: The University of Louisville's Institutional Repository. It has been accepted for inclusion in Electronic Theses and Dissertations by an authorized administrator of ThinkIR: The University of Louisville's Institutional Repository. This title appears here courtesy of the author, who has retained all other copyrights. For more information, please contact thinkir@louisville.edu. 


\title{
DEVELOPMENT OF A NEUROMUSCULOSKELETAL COMPUTER MODEL IN A CHONDRODYSTROPHIC DOG
}

\author{
By \\ Gregory James Russo States \\ B.S, University of Louisville, 2013 \\ A Thesis \\ Submitted to the Faculty of the \\ J.B Speed School of Engineering \\ in Partial Fulfillment of the Requirements \\ for the Degree of \\ Master of Engineering \\ In Bioengineering \\ Department of Bioengineering \\ University of Louisville \\ Louisville, Kentucky
}

April 2016 
April 12, 2016 


\section{DEVELOPMENT OF A NEUROMUSCULOSKELETAL COMPUTER MODEL IN A CHONDRODYSTROPHIC DOG}

\section{By}

Gregory James Russo States

B.S, University of Louisville, 2013

A Thesis Approved on

(Date)

By the Following Thesis Committee:

Dr. Gina E. Bertocci, Thesis Director

Dr. Dena R. Howland

Dr. David S.K. Magnuson

Dr. Angela K. Thompson

Dr. Nathan P. Brown, Dr. Gwendolyn J. Levine, and Dr. Jonathan Levine 


\section{ABSTRACT}

Intervertebral disc disease (IVDD) is a naturally occurring disease in dogs that produces a spontaneous injury to the spinal cord. IVDD is characterized by mineralization of the intervertebral disc nucleus pulposus, which reduces its load bearing capacity and results in high rates of intervertebral disc herniation (IVDH). IVDH is disproportionately present in Dachshunds compared to other breeds, affecting an estimated 1 in 5 Dachshunds during their lifetime (Levine, J. M. et al., 2011).

Assessment of injury severity and recovery in animal models is generally performed using a point scale, where subjects are graded according to metrics such as pain perception, joint movement, and limb coordination (Basso et al., 1995; Levine, G. J. et al., 2009; Olby, N. J. et al., 2001). Although these methods provide a general view of recovery, they are unable to quantify metrics such as joint motion/torque and muscle activation/force produced during specific phases of gait. OpenSim is an open source software package that allows users to estimate joint kinematics/torques and muscle forces/activations in a musculoskeletal model, which can be scaled to a subject's dimensions (Delp et al., 2007). Generic musculoskeletal models have been developed in the OpenSim platform for humans (Delp et al., 1990), cats (Keshner et al., 1997), and rats (Johnson et al., 2008), however to the author's knowledge no model has been developed for dogs. 
The purpose of the proposed study was to develop a subject-specific neuromusculoskeletal computer model of a healthy dog using OpenSim software (Delp, Anderson et al. 2007) to deduce patterns of muscle activity during locomotion. The longterm goal of this study is to utilize the model to inform rehabilitation strategies to enhance recovery and function in dogs with SCI based upon an improved understanding of muscle activation patterns. Additionally, the ability to characterize muscle activation patterns will provide a tool for quantifying the efficacy of therapeutic interventions in a canine model that could allow for potential therapeutic advancement in both dogs and humans.

The specific aims of this study were:

1. To characterize joint kinematics of healthy Dachshunds during walking gait.

2. To compare model-predicted joint kinematics to measured joint kinematics in healthy Dachshunds during walking gait.

H1: Pelvic limb joint range of motion of the model-predicted kinematics will not be different from kinematics calculated from marker trajectory data.

H2: Measured motion tracking marker trajectories will not be different from virtual model-predicted marker trajectories.

3. To quantify model sensitivity to changes in maximum muscle isometric force.

H3: Varying maximum muscle isometric force will affect peak muscle activation. 
To address these aims, a bilateral 3D model of the bony structures of the pelvis and pelvic limb (femur, tibia/fibula, phalanges, and metatarsals) and muscles was created using computed tomography (CT) imaging data. Parameters for the OpenSim model such as muscle origins and insertions, muscle cross-sectional area, and tendon slack length were obtained using computed tomography data or values from literature studies. Kinematic and kinetic data were incorporated in OpenSim to estimate joint kinematics and muscle activation patterns during locomotion.

In this study a subject-specific canine pelvic limb neuromusculoskeletal OpenSim model was developed based upon anatomically accurate data, as well as parameters of dogs described in literature. This model included representation of bilateral pelvic limb boney segments and muscles. This model was used to predict kinematics, muscle activation patterns and muscle forces during simulated gait. Findings illustrated that the model provided a reasonable approximation of joint kinematics as compared to measured joint kinematics, based on correlation coefficients calculated between modeled and measured joint kinematics and motion tracking marker trajectory data.

The extensor digitorum longus, tibialis cranialis, adductor, vastus lateralis/medialis, rectus femoris, and tensor fascia lata were primarily active during stance. The vastus lateralis/medialis, rectus femoris, tensor fascia lata, sartorius and gluteus medius were active during the first half of swing, while the adductor, semimembranosus, semitendinosus, and biceps femoris were active during the second half of swing. These activation patterns compare similarly with those found in the scientific literature, despite 
inherent differences in the comparison. This study illustrates the utility of an OpenSim model by demonstrating the ability to accurately model kinematic data, and predict muscle activation patterns during gait. Future work should involve further verification of modeled joint torques and muscle parameters, as well as describe small muscles not included in the current model. 


\section{TABLE OF CONTENTS}

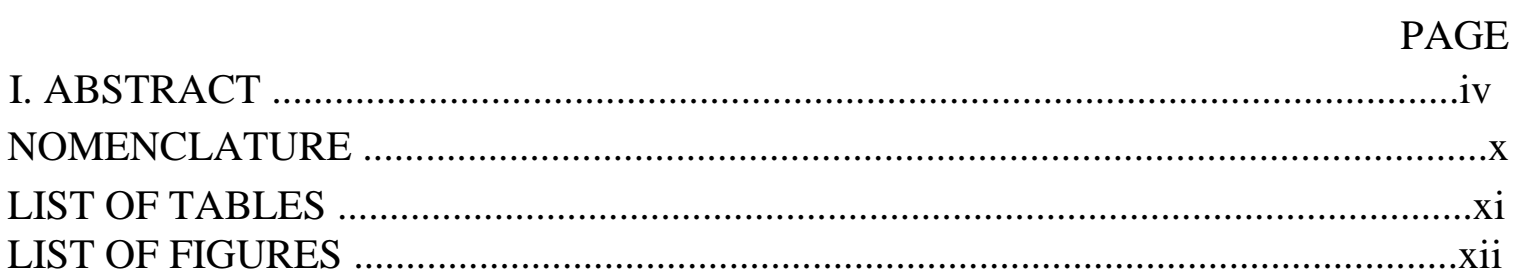

II. BACKGROUND AND SIGNIFICANCE …..................................................

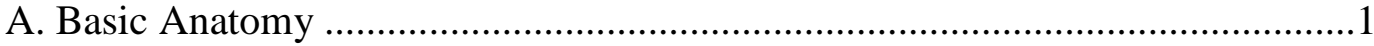

1. Vertebral Anatomy .........................................................................

2. Musculoskeletal Anatomy ................................................................ 3

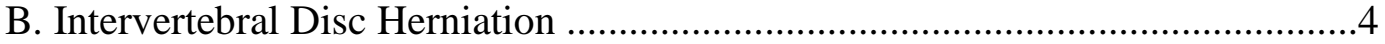

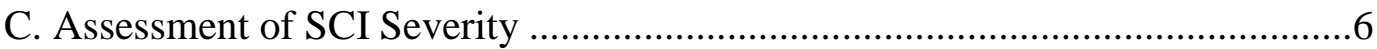

1. Assessment of SCI Severity Using a Grading Scale ............................ 7

2. Quantitative Assessment of SCI Severity .........................................10

a. Gait Analysis Using Discrete Variables .................................11

b. Functional Analysis .........................................................12

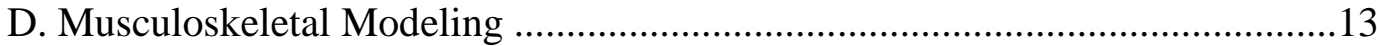

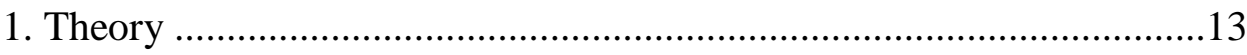

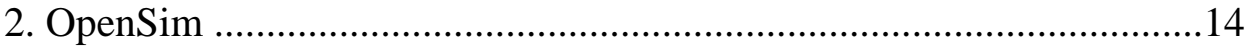

a. OpenSim Pipeline …........................................................15

b. Muscle Parameters ..............................................................19

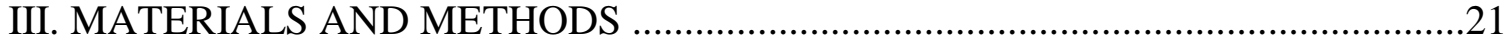

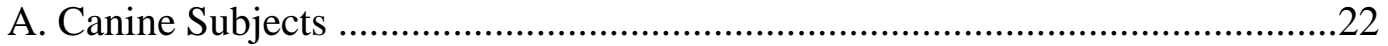

B. 3D Model of the Healthy Subject ..........................................................23

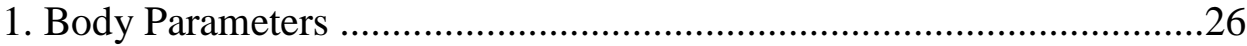

2. Muscle Parameters ............................................................................29

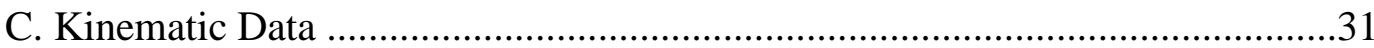


1. Motion Capture System ………………………………….................... 31

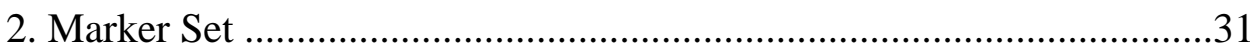

3. Kinematic and Kinetic Data Collection ...................................................32

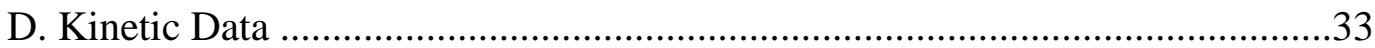

1. Ground Reaction Force Kinetic Data …………………………….......33

E. Incorporation of Kinematic and Kinetic Data into OpenSim .............................35

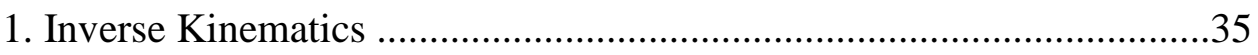

2. Residual Reduction Algorithm …………………….............................36

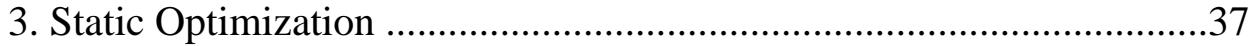

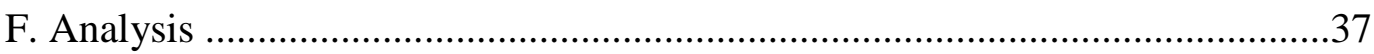

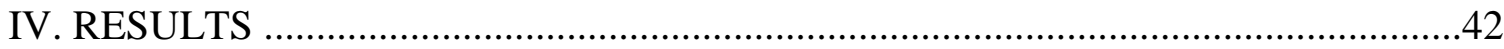

A. Computed Tomography …………………………….................................42

B. Kinematic Data of Healthy Subjects ...........................................................43

C. Model Kinematic Reconstruction Comparison to Motion Capture …………...47

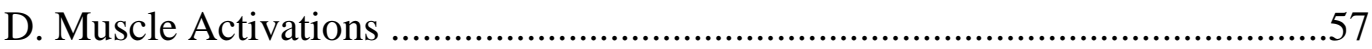

E. Parametric Sensitivity Analysis ...................................................................74

F. Residual and Ground Reaction Forces ………………………………….......78

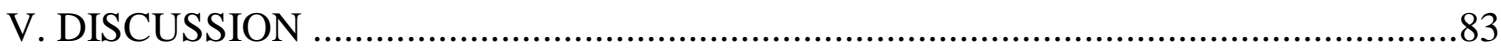

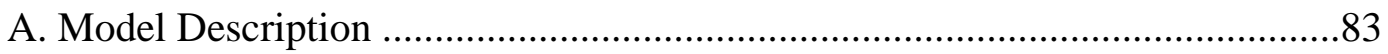

1. Joint Kinematics ................................................................................. 83

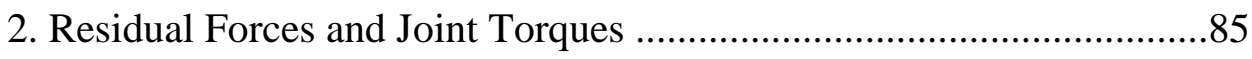

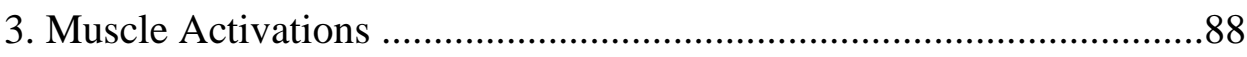

B. Parametric Sensitivity Analysis …………………………............................92

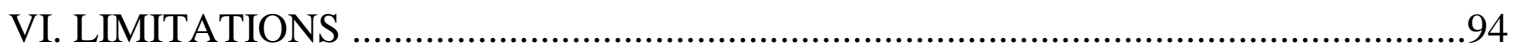

VII. RECOMMENDATIONS ………………………........................................98

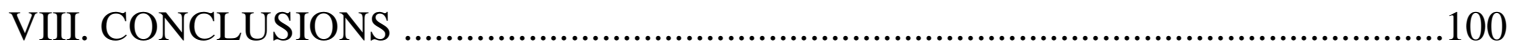

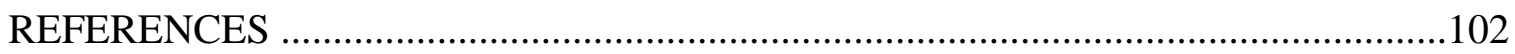


April 12, 2016

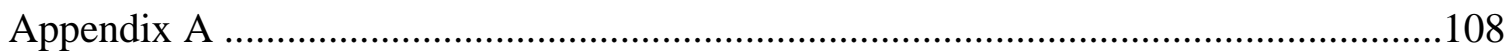

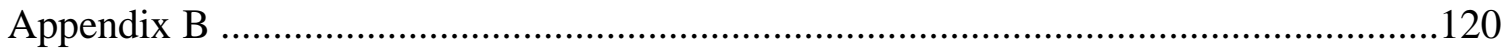

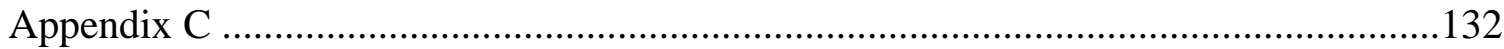

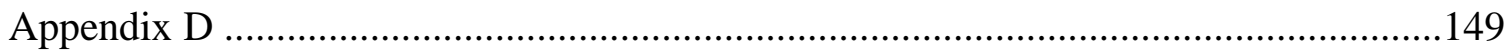




\section{NOMENCLATURE}

ACSA $=$ Anatomical cross-sectional area

$\mathrm{AF}=$ Annulus fibrosis

$\mathrm{CE}=$ Contractile element

$\mathrm{CSA}=$ Cross-sectional area

$\mathrm{CT}=$ Computed tomography

$\mathrm{EMG}=$ Electromyography

ISB = International Society of Biomechanics

IVD $=$ Intervertebral disc

IVDD $=$ Intervertebral disc disease

$\mathrm{IVDH}=$ Intervertebral disc herniation

$\mathrm{NP}=$ Nucleus pulposus

PCSA $=$ Physiological cross-sectional area

$\mathrm{PE}=$ Parallel element

$\mathrm{SCI}=$ Spinal cord injury

$\mathrm{SEE}=$ Series elastic element

$\mathrm{STL}=$ Stereolithography 


\section{LIST OF TABLES}

TABLE

PAGE

I. Muscles of the canine pelvis limb, their actions and innervation 3

II. 15 point grading scale to evaluate canine pelvic limb function ............................... 8

III. Pelvic limb function scores divided by injury severity at $0,2,4$, and 12 weeks postinjury

IV. Texas spinal cord injury score for dogs .10

V. Naming conventions, weight, gender, and age of subjects

VI. Muscle volumes and cross sectional areas from the healthy dog calculated using

Mimics 42

VII. Number of trials used to represent joints during each phase of gait

VIII. Average walking speed of healthy subjects

IX. Sensitivity index values for muscles during stance .75

X. Sensitivity index values for muscles during swing .76

XI. Peak joint torque and standard deviation in Healthy Subject 1 86

XII. Peak joint moment from various canine studies .87 


\section{LIST OF FIGURES}

FIGURE

PAGE

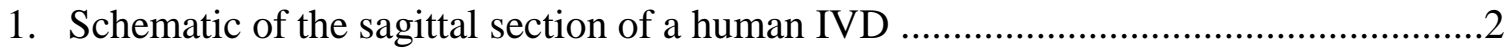

2. Hansen type I versus type II disc herniation ..........................................................

3. 3-element Hill-type muscle model ..........................................................................14

4. Diagram illustrating the basic composition of a muscle fiber ....................................20

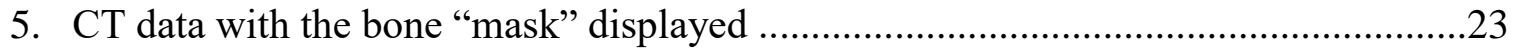

6. Three-dimensional representation of bones and muscles of the pelvic limb (ventral)

7. Three dimensional representation of bones and muscles of the pelvic limb (dorsal)

8. Three dimensional representation of bones and muscles of the pelvic limb (sagittal, left side) .25

9. Representative marker set used to acquire kinematic data .32

10. Vertical ground reaction force time history for a successful trial .34

11. Vertical ground reaction force time history for an unsuccessful trial ..........................35

12. An overview of the steps in creating a dynamic simulation in OpenSim ....................36

13. Inputs and outputs of the inverse kinematics step .......................................................36

14. Visualization of the model in the OpenSim platform ................................................43

15. Mean flexion/extension curves for the hip joint of individual subjects ......................45

16. Mean flexion/extension curves for the stifle joint of individual subjects ...................46

17. Mean flexion/extension curves for the tarsus joint of individual subjects ...................47

18. Hip flexion of a representative trial using JCS and OS methods ...............................48

19. Stifle flexion of a representative trial using JCS and OS methods .............................48

20. Tarsus flexion of a representative trial using JCS and OS methods ...........................49

21. Marker displacement of the iliac crest ...................................................................50

22. Marker displacement of the ischiatic tuberosity ....................................................

23. Marker displacement of the greater trochanter ……..................................................52

24. Marker displacement of the lateral femoral condyle .................................................53

25. Marker displacement of the tibial crest ..................................................................54 
26. Marker displacement of the lateral malleolus ......................................................55

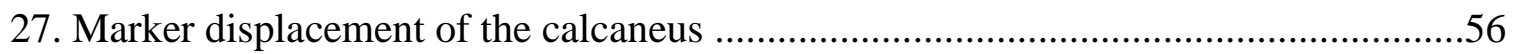

28. Marker displacement of the distal $5^{\text {th }}$ metatarsal .................................................57

29. Simulated muscle activations of the adductor magnus in the first healthy subject ....58

30. Simulated muscle activations of the biceps femoris in the first healthy subject ........59

31. Simulated muscle activations of the cranial tibial in the first healthy subject .60

32. Simulated muscle activations of the long digital extensor in the first healthy subject

33. Simulated muscle activations of the gastrocnemius in the first healthy subject .........62

34. Simulated muscle activations of the gemellus muscle in the first healthy subject .....63

35. Simulated muscle activations of the iliacus in the first healthy subject ......................64

36. Simulated muscle activations of the middle gluteal in the first healthy subject .........65

37. Simulated muscle activations of the quadratus femoris in the first healthy subject ...66

38. Simulated muscle activations of the rectus femoris in the first healthy subject .........67

39. Simulated muscle activations of the caudal Sartorius in the first healthy subject ......68

40. Simulated muscle activations of the cranial Sartorius in the first healthy subject ......69

41. Simulated muscle activations of the semimembranosus in the first healthy subject ..70

42. Simulated muscle activations of the semitendinosus in the first healthy subject .......71

43. Simulated muscle activations of the tensor fascia lata in the first healthy subject .....72

44. Simulated muscle activations of the vastus intermedius/lateralis in the first healthy

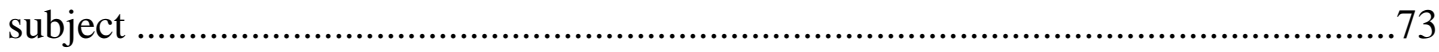

45. Simulated muscle activations of the vastus medialis in the first healthy subject .......74

46. Sensitivity index values for muscles during stance ...................................................76

47. Sensitivity index values for muscles during swing ....................................................77

48. Vertical ground reaction force for the right and left limb ........................................78

49. Vertical residual force during stance and swing ...................................................

50. Hip adduction moment of the right and left limb during stance and swing ................80

51. Hip flexion moment of the right and left limb during stance and swing .....................80

52. Hip rotation moment of the right and left limb during stance and swing ...................81

53. Stifle flexion moment of the right and left limb during stance and swing .................81

54. Tarsus flexion moment of the right and left limb during stance and swing ...............82 
55. Residual vertical force of the pelvis and vertical ground reaction force .86

56. Muscle activation of the left adductor magnus

.89

57. Values of muscle activation reported from (Wentink, 1976) and average values of estimated muscle activation 


\section{BACKGROUND AND SIGNIFICANCE:}

$\underline{\text { A. Basic Anatomy }}$

\section{Vertebral Anatomy}

The vertebral column in dogs is organized into five groups from rostral to caudal:cervical, thoracic, lumbar, sacral and caudal; the majority of IVDH ( 75\%) occurs between the eleventh thoracic $\left(T_{11}\right)$ and second lumbar $\left(L_{2}\right)$ vertebrae (Braund, 1994). The spinal cord is divided into segments: eight cervical, thirteen thoracic, seven lumbar, three sacral and approximately five caudal. All spinal cord segments are cranial to the vertebra of the same number except for the last two thoracic and first two lumbar segments, which are found entirely within the corresponding vertebrae. It should be noted that the functional spinal cord of the dog terminates near the $\mathrm{L}_{6}-\mathrm{L}_{7}$ range.

The intervertebral discs (IVD) are structures between the vertebrae that provide mechanical support and cushioning and allow slight movement of the spine (Shankar et al., 2009). IVD anatomy of humans and dogs is similar. The distinguishing features are the annulus fibrosus (AF) which encompasses the nucleus pulposus (NP); this entire structure has two cartilaginous endplates on each side (Lawson, 1958; Shankar et al., 2009). 


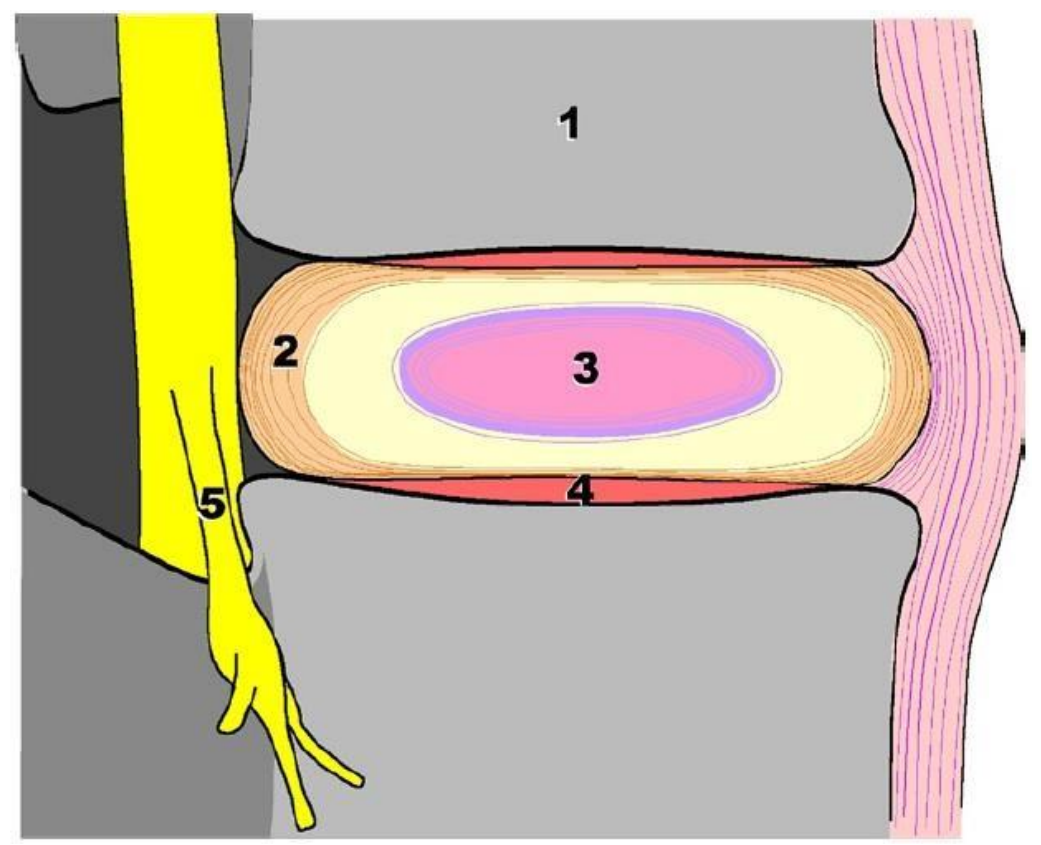

FIGURE 1 - Schematic of the sagittal section of a human IVD. 1: vertebral body, 2: annulus fibrosus, 3: nucleus pulposus, 4: endplate, 5: spinal nerve roots (Shankar et al., 2009).

The AF consists of 15-25 lamellae, which are concentric rings of collagen that vary in density (outer layers are thicker and more resistant to tensile forces) (Shankar et al., 2009). Elastin resides between each ring, which allows the intervertebral disc to recover its initial shape after deformation. Cells similar to fibroblasts also reside in the outer layer of the AF but become more oval towards the inner layers. Between the AF and NP resides a fibrous band of tissue called the transitional zone, seen in light yellow in Figure 4. Unlike the AF, the NP is composed of type II collagen and elastin organized around hydrophilic proteoglycans (versican, biglycan, decorin and others), which gives the overall structure a gel-like consistency. This consistency allows the NP to absorb compressive loading forces (Shankar et al., 2009). The NP also contains notochordal cells and chondrocyte-like cells, whose populations decrease and increase with age, 
respectively. These cells have few mitochondria, a large endoplasmic reticulum and vacuoles filled with osmotically active material (Risbud \& Shapiro, 2011).

\section{Musculoskeletal Anatomy}

Knowledge of the musculoskeletal system is necessary for interpreting the results of a musculoskeletal model. Knowledge of muscle action can be useful for identifying groups of muscles with similar actions, which allows the user to make inferences on the effects individual muscles have on large scale movement. Additionally, knowledge of muscle innervation can explain why certain muscles may be less active following a spinal cord injury. The muscles of the canine pelvic limb, as well as their role in locomotion and innervation are shown in Table I.

\section{TABLE I}

\section{MUSCLE OF THE CANINE PELVIC LIMB; THEIR ACTIONS AND INNERVATION}

(Evans \& deLahunta, 1988)

\begin{tabular}{|l|l|l|}
\hline Muscle & Action & Innervation \\
\hline Biceps femoris & Stifle extensor, hip extensor & Sciatic nerve (L6-S2) \\
\hline Semitendinosus & Hip extensor, stifle flexor & Sciatic nerve (L6-S2) \\
\hline Semimembranosus & Hip extensor & Sciatic nerve (L6-S2) \\
\hline Sartorius & Hip flexor & Femoral nerve (L4-L6) \\
\hline Gracilis & Hip extensor, stifle flexor & Obturator nerve (L4-L6) \\
\hline Pectineus & Limb adductor & Obturator nerve (L4-L6) \\
\hline $\begin{array}{l}\text { Adductor } \\
\text { (magnus/brevis) }\end{array}$ & Hip extensor, limb adductor & Obturator nerve (L4-L6) \\
\hline Tensor fascia lata & Hip flexion, stifle extension & $\begin{array}{l}\text { Cranial gluteal nerve (L6- } \\
\text { S1) }\end{array}$ \\
\hline Superficial gluteal & Hip extensor, limb adductor & $\begin{array}{l}\text { Caudal gluteal nerve (L7- } \\
\text { S2) }\end{array}$ \\
\hline Middle gluteal & $\begin{array}{l}\text { Hip extensor/abductor, pelvic limb } \\
\text { rotation (medial) }\end{array}$ & $\begin{array}{l}\text { Cranial gluteal nerve (L6- } \\
\text { S1) }\end{array}$ \\
\hline Deep gluteal & $\begin{array}{l}\text { Hip extensor/abductor, pelvic limb } \\
\text { rotation (medial) }\end{array}$ & $\begin{array}{l}\text { Cranial gluteal nerve (L6- } \\
\text { S1) }\end{array}$ \\
\hline
\end{tabular}


April 12, 2016

\begin{tabular}{|l|l|l|}
\hline Internal Obturator & Pelvic limb rotation (lateral) & Sciatic nerve (L6-S2) \\
\hline Gemellus & Pelvic limb rotation (lateral) & Sciatic nerve (L6-S2) \\
\hline Quadratus femoris & $\begin{array}{l}\text { Hip extension, pelvic limb rotation } \\
\text { (lateral) }\end{array}$ & Sciatic nerve (L6-S2) \\
\hline External Obturator & Pelvic limb rotation (lateral) & Obturator nerve (L4-L6) \\
\hline Quadriceps femoris ${ }^{1}$ & Stifle extension, hip flexion & Femoral nerve (L4-L6) \\
\hline Iliopsoas & Hip flexion & Femoral nerve (L4-L6) \\
\hline Cranial tibial & Tarsus flexion, paw rotation (lateral) & Peroneal nerve (L6,L7) \\
\hline Long digital extensor & Tarsus flexion, digit extension & Peroneal nerve (L6,L7) \\
\hline Peroneus longus & Tarsus flexion, paw rotation (medial) & Peroneal nerve (L6,L7) \\
\hline Gastrocnemius & Tarsus extension, stifle flexion & Tibial nerve (L7,S1) \\
\hline Superficial digital flexor & $\begin{array}{l}\text { Digit flexion, stifle flexion, tarsus } \\
\text { extension }\end{array}$ & Tibial nerve (L7,S1) \\
\hline Deep digital flexors & \\
\hline Popliteus & Digit flexion, tarsus extension & Tibial nerve (L7,S1) \\
\hline
\end{tabular}

${ }^{1}$ The quadriceps femoris is a muscle group that consists of the rectus femoris, vastus lateralis, vastus intermedius, and vastus medialis.

${ }^{2}$ The deep digital flexor group includes the flexor hallucis longus and flexor digitorum longus.

\section{B. Intervertebral Disc Herniation}

Intervertebral disc disease (IVDD) - synonymous with degenerative disc disease - affects the vertebra and can lead to intervertebral disc herniation (IVDH), which if left untreated can cause paraplegia and other long-term disabilities (Levine, G. J. et al., 2009). IVDD primarily mineralizes and stiffens the nucleus pulposus, which can lead to structural damage. Chondrodystrophic (“dwarf”) breeds are more susceptible to IVDH compared to non-chondrodystrophic dogs. One contributing factor is general disc morphology; chondrodystrophic dogs have a smaller NP in relation to the AF, which decreases NP functionality as a load bearing structure. In addition, notochordal and chondrocyte-like cells experience premature apoptosis in chondrodystrophic dogs through either intrinsic or extrinsic pathways, which leads to mineralization of the NP (Erwin et al., 2011). This is followed by mechanical failure of the disc, rupture of the 
annulus fibrosis and extrusion of the NP into the vertebral canal (Erwin et al., 2011). Discs bulge or rupture dorsally or posterolaterally, and compress nerve roots in the spinal cord (Erwin et al., 2011). Disc herniation may result from degenerate pieces of the NP migrating through tears in the AF (Erwin et al., 2011). Extrusion of the NP can occur either explosively or gradually, but both methods cause pressure on the spinal cord leading to partial or complete pelvic limb paralysis (Smolders et al., 2013). Explosive injuries are classified as Hansen Type I, and are more common in chondrodystrophic dogs. Gradual degeneration followed by partial AF ruptures is classified as Hansen Type II, and is more common in non-chondrodystrophic dogs (Hansen, 1951; Smolders et al., 2013). A visual representation of both types of degeneration can be seen in Figure 2.

\section{Hansen Type 1}
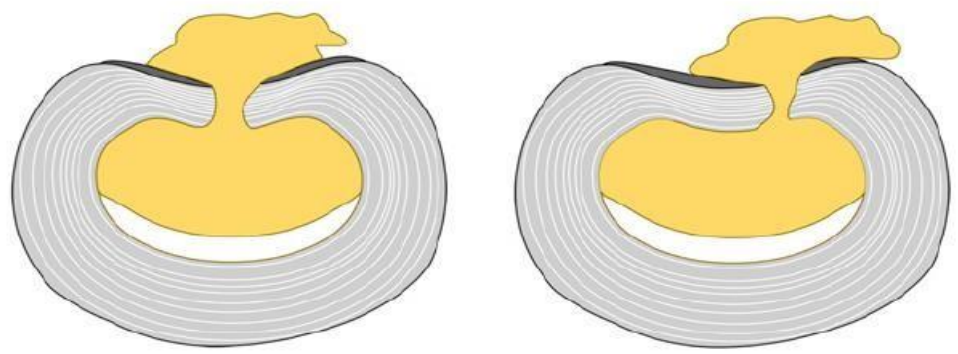

\section{Hansen Type 2}
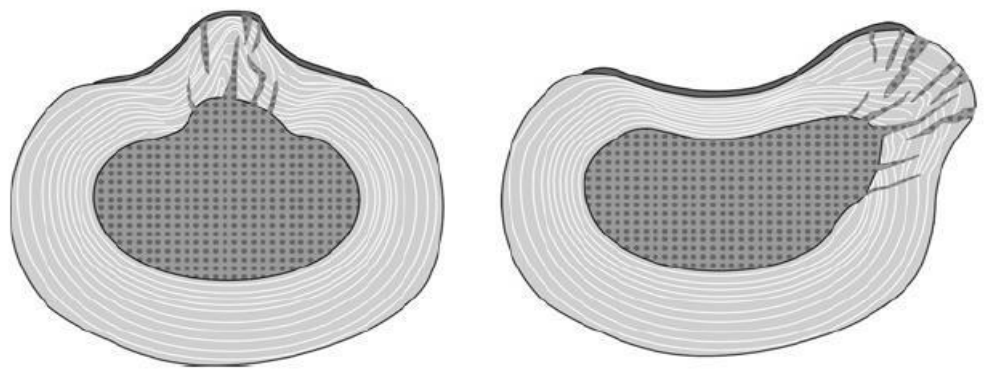

FIGURE 2 - Hansen type I versus type II disc herniation. Extrusion of the NP (yellow) through the AF (light grey) can be seen in type I, while type II herniation involves only partial ruptures and expansion of the AF (Smolders et al., 2013). 
An estimated 2\% of dogs develop IVDD during their lifetimes (Bergknut et al., 2012), but the risk is substantially higher - between 19 and $24 \%$ - for chondrodystrophic breeds (notably Dachshunds) (Levine, J. M. et al., 2011).

\section{Assessment of SCI Severity}

Assessment of recovery following SCI is often accomplished using grading scales to qualify relevant parameters, such as pain perception, weight support, and limb movement. Despite the wide use of these assessment tools, muscle recruitment and activation necessary to perform functional activities such as gait have not been described in dogs. Coordinated muscle activation patterns are responsible for generating limb movement and coordination of movement between limbs. Understanding the disruption of these coordinated muscle activation patterns in the pelvic limbs of dogs with SCI, along with compensatory strategies developed during recovery and functional reorganization can provide fundamental knowledge key to comprehending the process of voluntary motor control recovery. The ability to describe multi-muscle recruitment and activation patterns will allow for quantitative characterization of the SCI recovery process and identification of differences as compared to normal activation patterns, provide an evidenced based approach to designing rehabilitation and interventional strategies, and establish a comprehensive approach for evaluating putative therapies in future clinical trials. The following sections will describe some of the current methods to evaluate recovery following SCI.

\section{Assessment of SCI Severity Using a Grading Scale}


Functional gait scales that qualitatively characterize locomotion in animals have been developed; examples include the Basso, Beattie and Bresnahan (BBB) scale and the Texas Spinal Cord Injury Scale (TSCIS) (Basso et al., 1995; Levine, G. J. et al., 2009). Sensory scales have also been adapted for animal use, such as the modified Frankel scale (Frankel et al., 1969).

The grading criteria for the Frankel scale are similar to the ASIA scale; patients are classified by level of injury, type of injury, and completeness of injury. There are five levels (A: No motor or sensory function below the lesion, B: Sensation present below the lesion but no motor function, C: Some motor function present, but lacks characteristics such as weight support and coordination, D: Motor function intact enough to walk, with or without aids, and $\mathbf{E}$ : free of neurological symptoms) that determine level of injury completeness (Frankel et al., 1969). There is no test (such as the light touch and pinprick) to determine injury completeness; it is determined by the evaluator's judgment. Modified Frankel scales have been used for veterinary purposes, but like the original scale they lack the objectivity to evaluate qualifying spinal cord dysfunction (Levine, G. J. et al., 2009).

A study by Olby et al. in 2003 followed the recovery of 88 dogs who suffered thoracolumbar disc herniations over 12 weeks. Dogs were grouped into four groups: group 1 had the most severe injuries; groups 2 and 3 had moderate injuries while group 4 had only mild injuries. Gait was videotaped at 2, 4, and 12 weeks post-injury and qualitatively analyzed using the pelvic limb function grading system seen in Table II (Olby, N. et al., 2004). 


\section{TABLE II}

\section{5-POINT GRADING SCALE TO EVALUATE CANINE PELVIC LIMB FUNCTION}

(Olby, N. et al., 2004; Olby, N. J. et al., 2001).

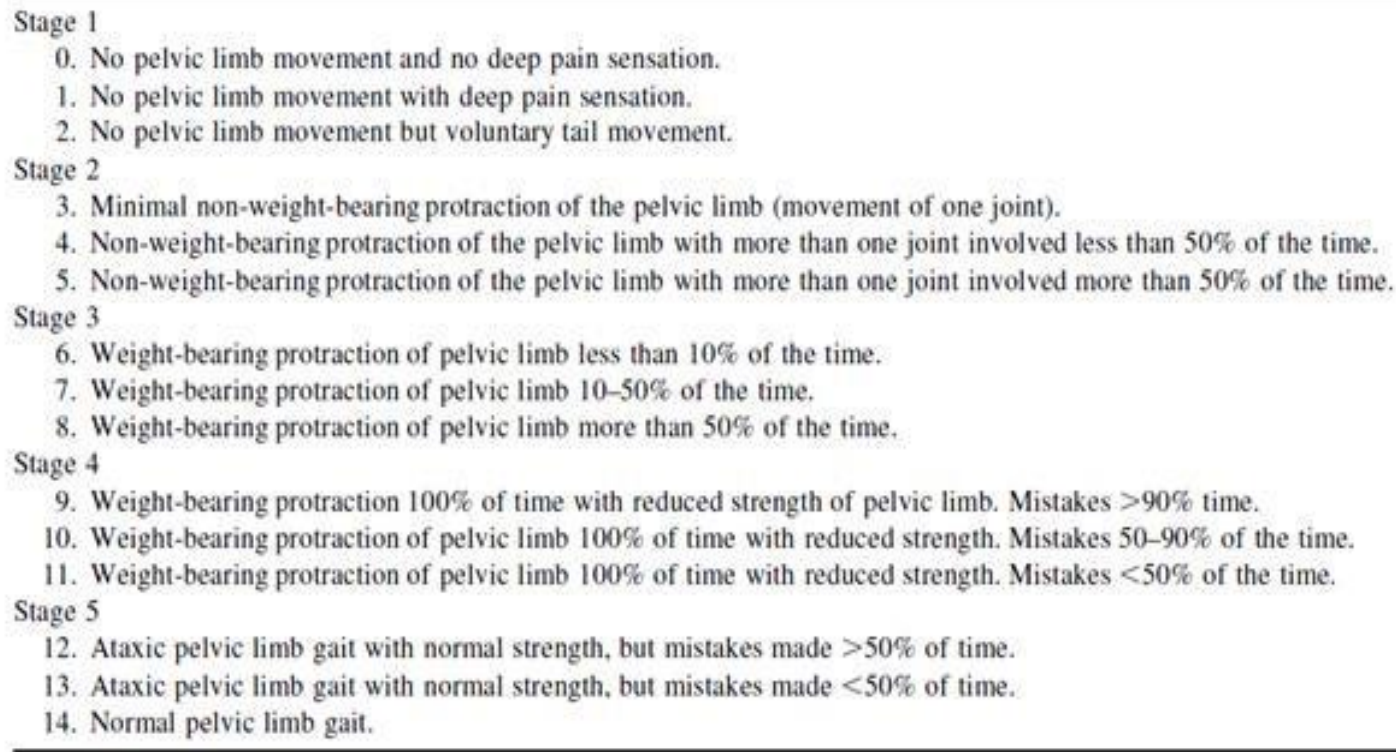

6. Weight-bearing protraction of pelvic limb less than $10 \%$ of the time.

7. Weight-bearing protraction of pelvic limb $10-50 \%$ of the time.

8. Weight-bearing protraction of pelvic limb more than $50 \%$ of the time. Stage 4

9. Weight-bearing protraction $100 \%$ of time with reduced strength of pelvic limb. Mistakes $>90 \%$ time.

10. Weight-bearing protraction of pelvic limb $100 \%$ of time with reduced strength. Mistakes $50-90 \%$ of the time.

11. Weight-bearing protraction of pelvic limb $100 \%$ of time with reduced strength. Mistakes $<50 \%$ of the time. Stage 5

12. Ataxic pelvic limb gait with normal strength, but mistakes made $>50 \%$ of time.

13. Ataxic pelvic limb gait with normal strength, but mistakes made $<50 \%$ of time.

14. Normal pelvic limb gait.

From Olby et al.. 2001.

Dogs in group 1 did not recover completely and showed a large variation in recovery (standard deviation of 4.75 points using the above grading scale at 12 weeks post-injury). Dogs in the moderate initial injury groups (2 and 3) showed similar levels of recovery, but few completely recovered at the 12 week time point. Dogs in the mild injury group all made a full recovery after 12 weeks, with some recovering fully after 4 weeks. A summary of the recovery patterns can be seen in Table III (Olby, N. et al., 2003). 
TABLE III

PELVIC LIMB FUNCTION SCORES DIVIDED BY INJURY SEVERITY AT 0, 2, 4, AND 12 WEEKS POST-INJURY, WHERE SD IS STANDARD DEVIATION, SEM IS STANDARD ERROR OF THE MEAN, AND N IS THE NUMBER OF OBSERVATIONS FOR EACH DATA POINT (Olby, N. et al., 2003)

\begin{tabular}{lcllll}
\hline $\begin{array}{l}\text { Group: time } \\
\text { point (weeks) }\end{array}$ & Median score (S) & SD & SEM & Range & $N$ \\
\hline Group 1:0 & $0(0)$ & 0 & 0 & 0 & 25 \\
$1: 2$ & $5(35.71)$ & 3.65 & 0.89 & $0-9.25$ & 17 \\
$1: 4$ & $8(57.14)$ & 4.31 & 0.94 & $0-12.5$ & 21 \\
$1: 12$ & $9.5(67.86)$ & 4.75 & 1.09 & $0-13$ & 19 \\
Group 2:0 & $1(7.14)$ & 0.21 & 0.05 & $0.5-1$ & 19 \\
$2: 2$ & $8.5(60.71)$ & 3.25 & 0.84 & $3.25-12.75$ & 15 \\
$2: 4$ & $9.5(67.86)$ & 2.28 & 0.59 & $5.25-13$ & 15 \\
$2: 12$ & $11.25(80.36)$ & 1.92 & 0.55 & $8.75-14$ & 12 \\
Group 3:0 & $4(28.57)$ & 0.83 & 0.14 & $2-5$ & 33 \\
$3: 2$ & $9(64.29)$ & 2.62 & 0.5 & $3.5-13.25$ & 27 \\
$3: 4$ & $10.5(75)$ & 2.24 & 0.45 & $5.25-14$ & 25 \\
$3: 12$ & $12(85.71)$ & 1.57 & 0.33 & $9.25-14$ & 23 \\
Group 4:0 & $8.25(58.93)$ & 1.99 & 0.60 & $6-13$ & 11 \\
$4: 2$ & $11.75(83.93)$ & 1.13 & 0.38 & $10-13.25$ & 9 \\
$4: 4$ & $12.875(91.96)$ & 1.4 & 0.44 & $11-14$ & 10 \\
$4: 12$ & $14(100)$ & 0.78 & 0.25 & $12-14$ & 10 \\
\hline
\end{tabular}

The Texas Spinal Cord Injury Score (TSCIS) proposed by Levine et al. in 2009 attempts to address this limitation (Levine, G. J. et al., 2009). It scores each limb based on three criteria: gait, proprioceptive positioning and nociception (Frankel et al., 1969) (Table IV). This method increases the number of evaluation criteria and arguably describes dysfunction/function more accurately, however it is still subject to the limitation of qualitative evaluation (user interpretation, intra-observer variability, etc.). Additionally, although it describes a dog's ability to create movement it does not describe the underlying muscle activation necessary to create these movements. An OpenSim 
model has the ability to provide detailed descriptions of recovery metrics, which allows researchers a greater understanding of the processes involved in rehabilitation.

\section{TABLE IV}

TEXAS SPINAL CORD INJURY SCORE FOR DOGS (Levine, G. J. et al., 2009).

Texas Spinal Cord Injury Score (TSCIS) for dogs.

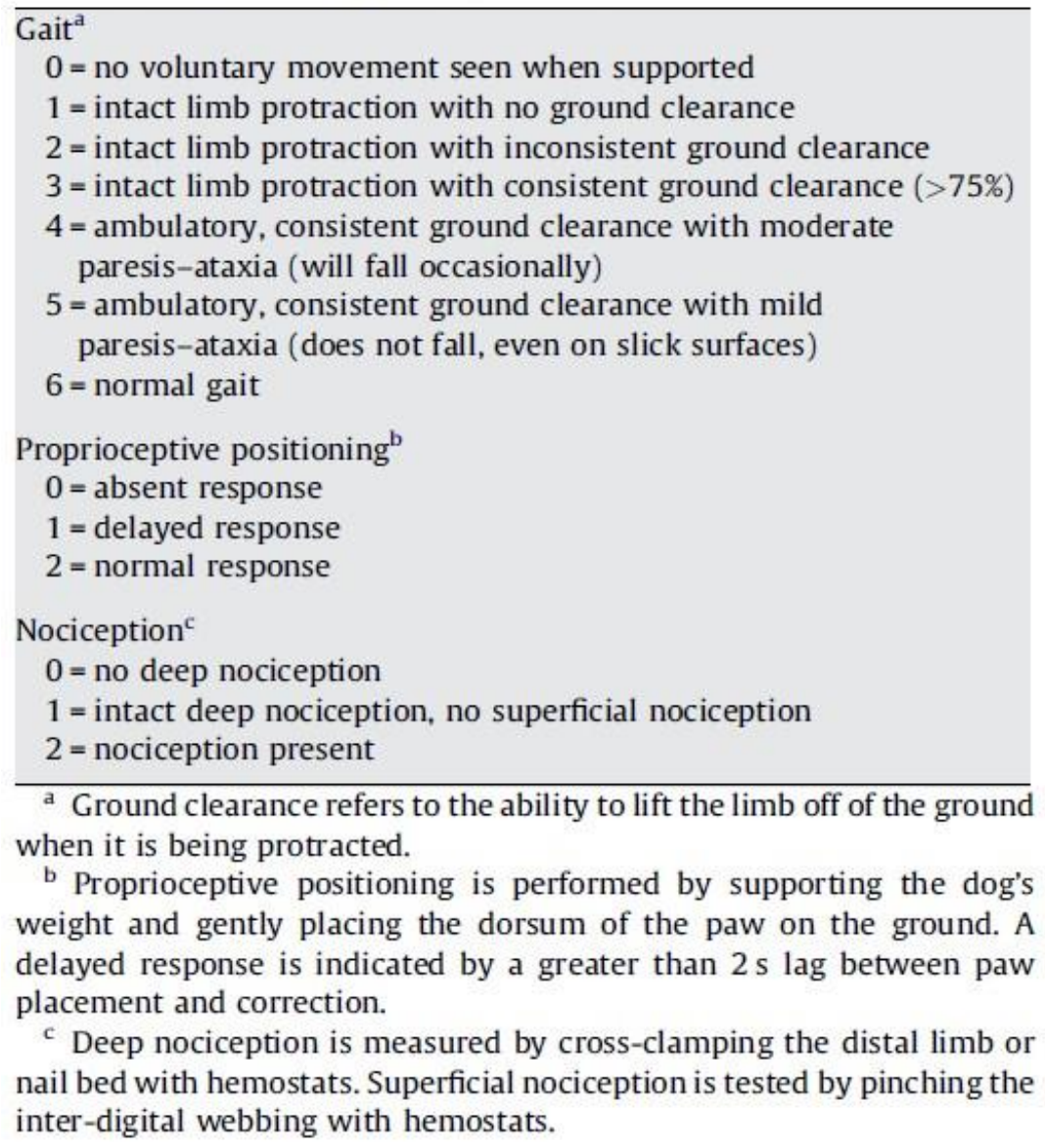

\section{Quantitative Assessment of SCI Severity}

The techniques described in section 2.3.1 rely on scores determined by evaluators, and are therefore subject to inter-rater reliability issues. Conversely, quantitative methods 
of gait assessment utilize measurable quantities such as kinematic variables (i.e. joint angles or paw trajectories) and/or kinetic variables (such as ground reaction force) to determine the extent of recovery. These methods eliminate user subjectivity, but tend to focus on one aspect of movement instead of presenting an overall picture of recovery.

\section{a. Gait Analysis Using Discrete Variables}

Previous quantitative analysis on dogs with spinal cord injury has used various metrics to define recovery. For example, a study by Jeffery et al. defines the mean diagonal coupling interval as a criteria to define coordination of thoracic and pelvic limb movement (Hamilton, Lindsay et al., 2007). Motion capture data was initially obtained from dogs of various breeds and sizes, then from dogs with spinal cord injury. Paw contact was determined using a custom MATLAB script which detected the maximum and minimum sagittal plane paw position during each gait cycle. The time difference between each successive thoracic and pelvic paw strike was calculated and summed, then divided by the total number of steps, giving the mean diagonal coupling interval. Results showed that mean diagonal coupling interval is independent of animal size, gait speed, or orthopedic disease. However, it is sensitive to changes of gait seen in dogs with spinal cord injury (Hamilton, Lindsay et al., 2007).

Another quantitative metric to assess spinal cord injury is the coefficient of variation in paw placement. This metric was also used by Jeffery et al. to define the degree of variability of paw placement in both the thoracic and pelvic limbs (Hamilton, L. et al., 2008). To calculate coefficient of variation, the distance between consecutive paw placements in the sagittal plane was calculated during each gait cycle (for both thoracic and pelvic limbs). These values were organized by pelvic and thoracic limb, then 
the mean and standard deviation was calculated for each trial. Coefficient of variation was defined as the standard deviation divided by the mean. The rationale for this metric is that spinal cord injured dogs will exhibit greater variability in paw placement, so the standard deviation will be a more descriptive and sensitive measure (Hamilton, Lindsay et al., 2007).

\section{b. Functional Analysis}

In gait analysis, discrete outcomes such as range of motion (ROM) or maximum joint angles are determined. Despite their relevance, discrete outcomes describe only one aspect of a curve rather than the overall variation of curve shape (Donoghue et al., 2008). To address the limitation of using discrete outcomes to describe gait, various methods have been used to characterize the time history of these outcomes (i.e. joint angle throughout the gait cycle). Functional data analysis (FDA) is a statistical means for quantifying differences in data that are in the form of a curve, waveform or function, such as joint angle time history during gait (Ramsay, J. O. S., B.W., 2005). FDA is particularly useful for isolating and analyzing temporal characteristics in a data set, notably derivatives or rates of change. The ability to define differences in these features without sacrificing statistical power or temporal resolution is particularly relevant to kinematic and kinetic analysis of motion, given that ground reaction force and joint angle data are functions of time. Using FDA it is possible to quantify differences in limb movement that would otherwise not be identified when using only discrete outcomes such as ROM or maximum joint angles (Harrison, 2014). This method allows users to apply a statistical method to describe more general patterns in movement. 
Functional analysis is performed on waveforms by transforming time-series data into a smaller number of components outside of the time domain. Methods such as Fourier transformation, spline approximation, and wavelet decomposition have been used for this purpose (Duhamel et al., 2006; Marghitu \& Nalluri, 1997; Ramsay, J. O. S., B.W., 2002). Each transformation has unique advantages and disadvantages; therefore the researcher must carefully choose which transformation to use depending on the type of waveform in question. For example, a Fourier series transformation is a powerful tool for signal analysis, although the transformation has some inherent limitations. Notably, any change in a signal is represented as an average change in frequency, which implies that the location and timing of fluctuations in a waveform cannot be determined (Marghitu \& Nalluri, 1997). Functional data analysis using principal components and basis function expansion (Ramsay, J. O. H., G; Graves, S, 2009; Ramsay, J. O. S., B.W., 2005) overcomes this limitation and has been applied in multiple studies to analyze joint angle kinematics (Coffey et al., 2011; Daffertshofer et al., 2004; Dona et al., 2009; Donoghue et al., 2008; Ryan et al., 2006) by comparing the covariance between data sets that are functions of time. The principal components are iteratively-calculated, linear combinations of the original data where each successive component is constrained to be orthogonal to the previous component. Approximately $95 \%$ of variance can be captured within the first four principal components, which reduces the number of components necessary to draw significant conclusions compared to analyzing each point from the original data (Dona et al., 2009; Donoghue et al., 2008; Ryan et al., 2006).

\section{Musculoskeletal Modeling}

\section{Theory}


Modeled muscle action must be realistically representative of muscle behavior while reducing the system to a solvable number of components. One of the earliest models still employed today is the three-element musculo-tendon model, a mechanical analog that approximates muscle length-force-velocity relationships (Hill, 1938). This model contains a contractile element (CE), a passive element (PE), and a series elastic element (SEE). The CE and PE both contribute to muscle force $\left(\mathrm{F}^{\mathrm{m}}\right)$ depending on the muscles instantaneous length, velocity, and activation (Zajac, 1989). The SEE represents energy stored in muscle cross bridges. An example of this model can be seen in Figure 3.

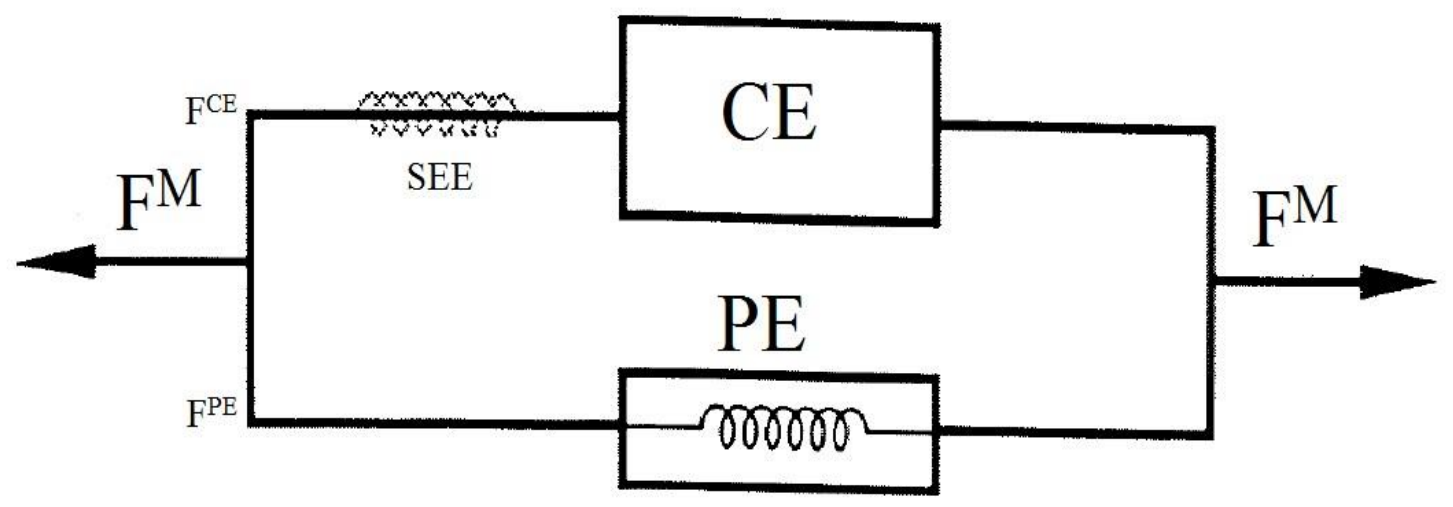

FIGURE 3 - 3-element Hill-type muscle model, where CE is the contractile element, PE is the passive element, and SEE is the series elastic element. $\mathrm{F}^{\mathrm{M}}$ represents the overall muscle force, while $\mathrm{F}^{\mathrm{CE}}$ and $\mathrm{F}^{\mathrm{PE}}$ represent the forces from the contractile and passive element respectively (Zajac, 1989).

\section{OpenSim}

OpenSim is an open source software program developed by the Departments of Bioengineering and Mechanical Engineering at Stanford University that models and 
simulates motion of the musculoskeletal system (Delp et al., 2007). This software was developed to overcome two major limitations in experimental dynamic movement studies: 1) variables such as forces generated by muscles are not measurable, and 2) establishment of relationships between muscle activation and limb movement is difficult to determine from experimental data alone. Differential equations adapted from a study by Thelen are used to describe muscle contraction dynamics and body segmental dynamics (Delp et al., 2007; Thelen, 2003). OpenSim uses these equations to dynamically simulate a musculoskeletal model and determine patterns of muscle activity associated with a coordinated movement (Delp et al., 2007). In other words, model simulation dynamically solves for muscle forces and activation patterns required to complete a musculoskeletal task by using biomechanical relationships between muscles, bones and connective tissues. The calculated forces and movements are optimized using a weighted least squares algorithm that minimizes deviations from kinematic data and reduces residual forces.

\section{a. OpenSim Pipeline}

A series of steps must be taken before solving for individual muscle contributions. First, a model of the biological system must be created. Bones are modeled as rigid bodies, while muscles and tendons are modeled as linear actuators. The model parameters are often determined using anatomically correct data (generally from medical imaging); this includes length and mass of bones, their attachment points to other rigid bodies in the model via joints, and muscle origins and insertions. Furthermore, muscles are constrained by parameters such as maximum isometric force, optimal fiber length, tendon slack 
length, and pennation angle; these parameters affect maximum and minimum joint angles as well as general muscle contraction dynamics (Xiao \& Higginson, 2010). Individual subjects can be represented using the generic human musculoskeletal model by using the OpenSim scaling function. To accomplish scaling, a static trial of the subject fitted with a marker set corresponding to the model marker set is recorded. The model is scaled so that virtual model markers best match measured subject markers. Features such as bone length, mass, and moments of inertia, as well as muscle origins and insertions are also scaled accordingly (Delp et al., 2007).

The second step solves an inverse kinematics problem using a least-squares equation that reduces the overall difference between the measured kinematic data coordinates and the model virtual coordinates for each marker. Each frame is analyzed and solved to minimize the mean squared error using

$$
\text { Squared Error }=\sum_{i=1}^{\text {markers }} w_{i}\left(x_{i}^{\text {subject }}-x_{i}^{\text {model }}\right)^{2}+\sum_{j=1}^{\text {joint angles }} w_{j}\left(\theta_{j}^{\text {subject }}-\theta_{j}^{\text {model }}\right)^{2}
$$

where $x_{i}^{\text {subject }}$ and $x_{i}^{\text {model }}$ are positions of the $i$ th marker for the subject and model, respectively, $\theta_{\mathrm{j}}^{\text {subject }}$ and $\theta_{\mathrm{j}}^{\text {model }}$ represent the values of the $j$ th joint angle, and $\omega_{i}$ and $\omega_{j}$ are weighting factors (Delp et al., 2007). The outcomes of this step are joint angles and displacements for all model bodies.

The third step incorporates measured ground reaction forces (kinetic data) into the model with a residual reduction algorithm that drives the kinetics calculated using inverse kinematics to be consistent with measured external forces, using 


$$
\vec{F}_{\text {external }}=\sum_{i=1}^{\text {segments }} m_{i} \vec{a}_{i}-\vec{F}_{\text {residual }}
$$

where $\mathrm{F}_{\text {external }}$ is the net combination of all external forces, $\mathrm{F}_{\text {residual }}$ is the residual force, $\mathrm{a}_{\mathrm{i}}$ is the acceleration of the center of mass of the $i$ th body segment, and $\mathrm{m}_{\mathrm{i}}$ is the mass of the $i$ th body segment (Delp et al., 2007). Error in input kinematics/kinetics (e.g. marker motion artifact and electrical noise) can cause inconsistencies in the dynamics calculated using kinematics alone to model movement. The residual reduction algorithm performs slight iterative adjustments to mass distribution properties and joint angle kinematics of the model to reduce $F_{\text {residual. }}$.

In theory, the residual force should be zero (i.e. in the case of no measurement or model error). Because measurement error is an inherent limitation in mechanical systems, Step 3 attempts to reduce the residual error over the course of the motion simulation by iteratively making small adjustments to motion trajectory and mass parameters (Delp et al., 2007).

The fourth step computes the muscle excitation patterns necessary to create the desired movement. The generic musculo-tendon model is used as a basis for creating any in silico muscle model. Muscles in OpenSim are based off of the equations proposed by Thelen, which uses two nonlinear differential equations to describe activation and contraction dynamics (Thelen, 2003). Activation dynamics are modeled using a muscle excitation signal coefficient $(u)$, a dimensionless measure between 0 and 1 to represent minimum and maximum excitation. This parameter is related to muscle activation using

$$
\frac{d a}{d t}=\frac{u-a}{\tau_{a}(a, u)}
$$


where $a$ is muscular activation, $\tau_{\mathrm{a}}(a, u)$ is a time constant that varies with activation magnitude and direction by

$$
\tau_{a}(a, u)=\left\{\begin{array}{cc}
\tau_{a c t}(0.5+1.5 a) ; & u>a \\
\tau_{\text {deact }} /(0.5+1.5 a) ; & u \leqslant a
\end{array}\right.
$$

where $\tau_{\text {act }}$ and $\tau_{\text {deact }}$ are the activation and deactivation time constants, respectively (Thelen, 2003).

A differential equation to model contraction dynamics (Zajac, 1989) was simplified by Thelen to an ordinary linear equation with two states (Thelen, 2003). First, it should be noted that the active force-length relationship of a muscle was represented by

$$
f_{l}=e^{-\left(\overline{L^{M}}-1\right)^{2} / \gamma}
$$

where $f_{l}$ is a force-length scale factor, $\bar{L}^{M}$ is the normalized fiber length, and $\gamma$ is a constant which was determined to be 0.45 (Thelen, 2003). Muscle fiber velocity $\left(V^{M}\right)$ was then defined as

$$
V^{M}=(0.25+0.75 a) V_{\max }^{M} \frac{\overline{F^{M}}-a f_{l}}{b}
$$

where $V_{\max }^{M}$ is the maximum contraction velocity and $\bar{F}^{M}$ is the active muscle force (Thelen, 2003). The parameter $b$ is dependent on the state of the muscle fiber (whether it is shortening or lengthening), and defined by

$$
b=\left\{\begin{array}{cc}
a f_{l}+\overline{F^{M}} / A_{f} ; & \overline{F^{M}} \leqslant a f_{l} \\
\frac{\left(2+2 / A_{f}\right)\left(a f_{l} \bar{F}_{\text {len }}^{M}-\overline{F^{M}}\right)}{\left(\bar{F}_{\text {len }}^{M}-1\right)} ; & \overline{F^{M}}>a f_{l}
\end{array}\right.
$$


where $\bar{F}_{l e n}^{M}$ is the maximum possible force when the muscle is lengthening, and $A_{F}$ is a constant determined to be 0.25 (Thelen, 2003).

\section{b. Muscle Parameters}

Inherent parameters in the OpenSim muscle model are optimal fiber length, maximum isometric force, pennation angle, tendon slack length, and maximum contraction velocity.

The amount of force a muscle can produce varies depending on the amount of overlap between actin and myosin filaments in each sarcomere within the muscle fiber. Overlap of actin and myosin filaments is determined by the length of the individual muscle fiber during activation. Consider the muscle fiber shown in Figure 4 where $l^{\mathrm{s}}$ is the length of a sarcomere, $\mathrm{l}^{\mathrm{M}}$ is fiber length, and $\mathrm{F}$ is an applied force to each end of the muscle fiber. Fiber length and sarcomere length increase with increasing force; sarcomere length determines the amount of actin and myosin overlap. At shorter and longer lengths actin and myosin overlap is minimal and maximal; both of these scenarios produce minimal force output. However, there is a length at which actin and myosin overlap is ideal, called the optimal muscle fiber length (Zajac, 1989). 


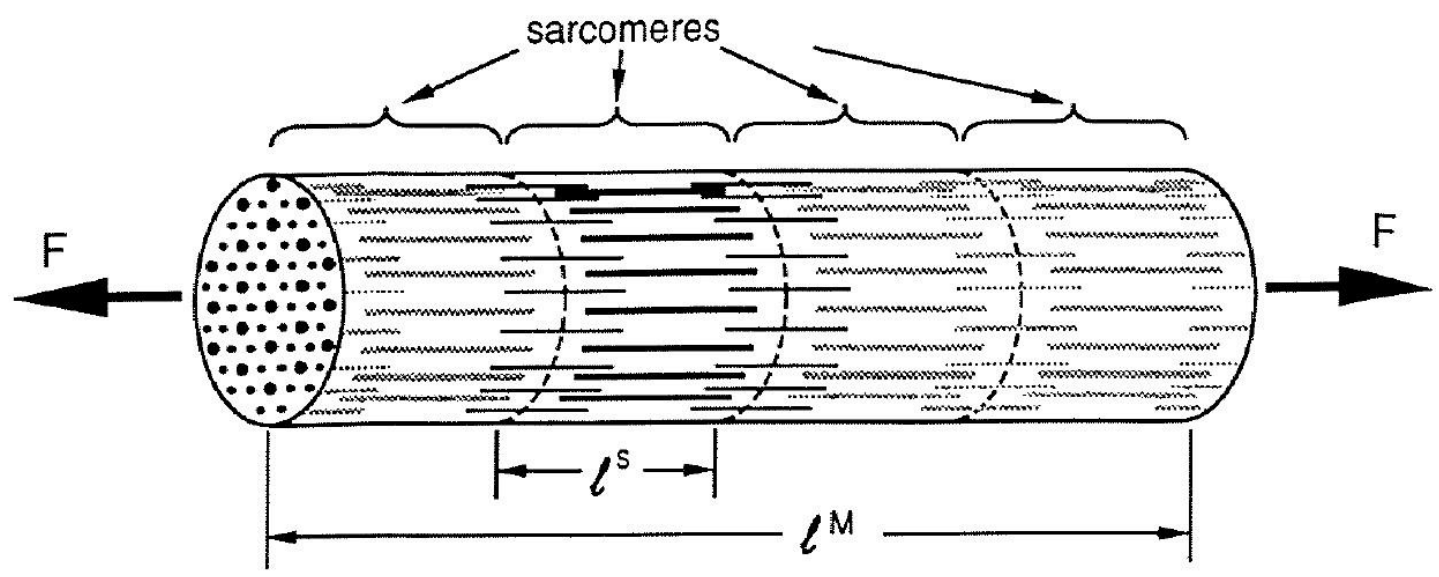

FIGURE 4 - Diagram illustrating the basic composition of a muscle fiber where $\mathrm{F}$ is an applied force to each end of the muscle fiber, $\mathrm{l}^{\mathrm{M}}$ is fiber length, and $\mathrm{l}^{\mathrm{s}}$ is sarcomere length (Zajac, 1989).

Maximum isometric force is the largest amount of force an individual muscle can produce during isometric contraction. This is determined by the physiological muscle cross sectional area (PCSA) opposed to the anatomical cross sectional area (ACSA), which accounts for the muscle cross sectional area as well as the arrangement of fibers within a muscle. This arrangement of fibers - known as pennation angle - determines how much force a muscle can exert along its line of action. Muscles can be either pennated - when muscle fibers are at an acute angle relative to the tendon - or parallel fibered (Zajac, 1989). A detailed description of these terms can be found in section 3.1.1.2.

Fully activated muscle fibers will quickly shorten and then immediately stop; the length at which it stops corresponds to a velocity based on force-velocity relations (Zajac, 1989). Maximum contraction velocity refers to this velocity in which in which a muscle can no longer maintain tension. Maximum velocity $\left(\mathrm{V}_{\max }\right)$ is approximated to be 


$$
v_{\max }=10 l^{M} m s^{-1}
$$

where $\mathrm{l}^{\mathrm{m}}$ is the muscle fiber length (Anderson \& Pandy, 1999). Finally, it should be noted that tendon slack length determines the length at which a tendon starts producing force. A tendon can be stretched to a certain length before applying force, which is the tendon slack length. This parameter is not well characterized in the literature due to difficulties in obtaining accurate in vitro and in vivo measurements.

\section{MATERIALS AND METHODS}

Subject specific anatomical data acquired from computed tomography (CT) imaging of a two-year-old female Dachshund $(5.35 \mathrm{~kg})$ with no evidence of neurologic disease was used to develop a representative three dimensional computer model of the pelvis and pelvic limbs. Model parameters determined from the CT scan (bone geometry, segment mass, muscle PCSA, joint center locations, and muscle attachment points) were combined with parameters estimated from literature values (muscle fiber length and tendon slack length) to create a musculoskeletal model that was implemented in the OpenSim platform (Stanford University, Stanford, CA).

Modeling joint angles and muscle activations was accomplished by: (1) collecting kinematic and kinetic data from three healthy dogs, including the dog used to build the model, (2) performing an inverse kinematics analysis to simulate motion from collected kinematic data, (3) performing a residual reduction analysis to reduce discrepancies between modeled kinematics and measured ground reaction forces, and (4) combining the 
simulated motion from the inverse kinematics analysis with the kinetic data to perform a static optimization analysis.

Joint angles and muscle activation were evaluated for all three healthy dogs. Joint angles determined from computer model simulation were compared to joint angles determined from motion capture kinematic data to verify simulation outcomes. Additionally, a parametric sensitivity analysis was performed using kinematic and kinetic data obtained from the first healthy subject.

\section{$\underline{\text { A. Canine Subjects }}$}

Parameters for the model were obtained from a two-year-old female Dachshund $(5.35 \mathrm{~kg})$ with no evidence of neurologic disease. Kinematic data was obtained from the aforementioned dog, a three-year-old male Dachshund (5.44 kg) and a four-year-old female Dachshund $(6.44 \mathrm{~kg})$. Owner consent was obtained prior to data collection, and all procedures involving the canine subject were performed in agreement with an approved Institutional Animal Care and Use Committee protocol (AUP \#2013-150).

\section{TABLE V}

NAMING CONVENTIONS, WEIGHT, GENDER, AND AGE OF SUBJECTS

\begin{tabular}{|c|c|c|c|}
\hline Subject Name & Weight (kg) & Gender & Age (years) \\
\hline Healthy Subject 1 & 5.35 & Female & 2 \\
\hline Healthy Subject 2 & 5.44 & Male & 3 \\
\hline Healthy Subject 3 & 6.44 & Female & 4 \\
\hline
\end{tabular}




\section{B. 3D Model of the Healthy Subject}

The initial model was developed using computed tomography data (slice thickness 1 $\mathrm{mm}$, resolution $0.375 \mathrm{~mm} \times 0.375 \mathrm{~mm} / \mathrm{pixel})$. Imaging was conducted by the College of Veterinary Medicine and Biomedical Sciences at Texas A\&M University .

Images obtained from the CT scan were imported into medical imaging and processing software ${ }^{1}$. Tissues were differentiated based on Hounsfield intensity values, which were initially set according to human preset thresholds and adjusted until a clear distinction between bone, muscle, and fat was observed.
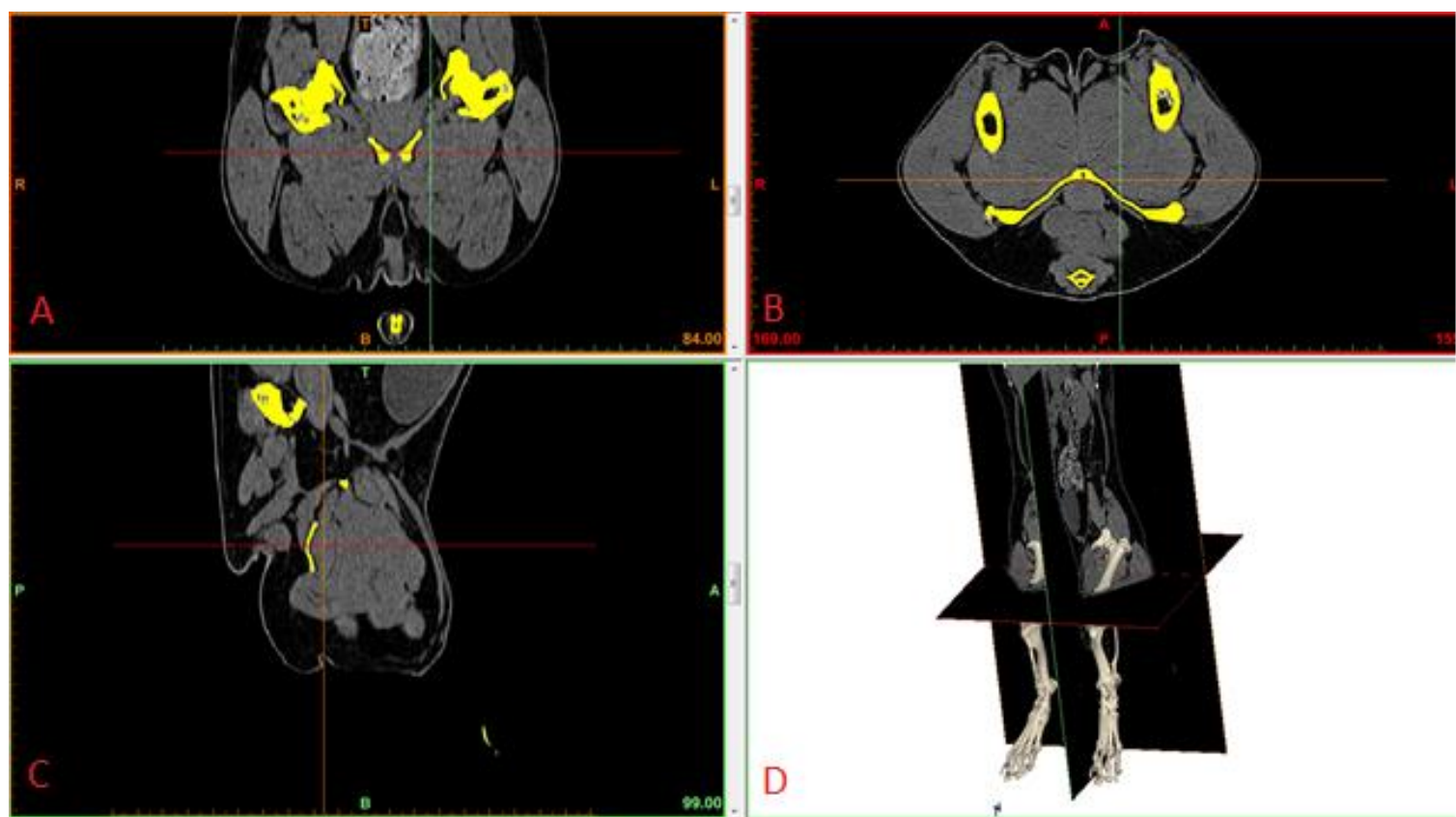

FIGURE 5 - CT data with the bone "mask" displayed (yellow), viewed from the frontal plane (A), transverse plane (B), and sagittal plane (C). The three orthogonal views are overlaid on a three dimensional bone reconstruction (D).

\footnotetext{
${ }^{1}$ Mimics, Materialise NV, Leuven, Blegium
} 
Final Housenfield threshold ranges were cortical bone (>662), trabecular bone (226 to 661) muscle (-69 to 225), and fat (-205 to -70). A “mask" was created for each tissue which stored the locations of pixels within each threshold range (Figure 5). Individual bones and muscles were defined by creating borders in the appropriate mask by hand tracing observable boundaries in each individual slice based on a comprehensive guide of canine skeletal and muscle anatomy (Evans \& deLahunta, 1988). A threedimensional representation of the bones and muscles can be seen in Figures 6-8.

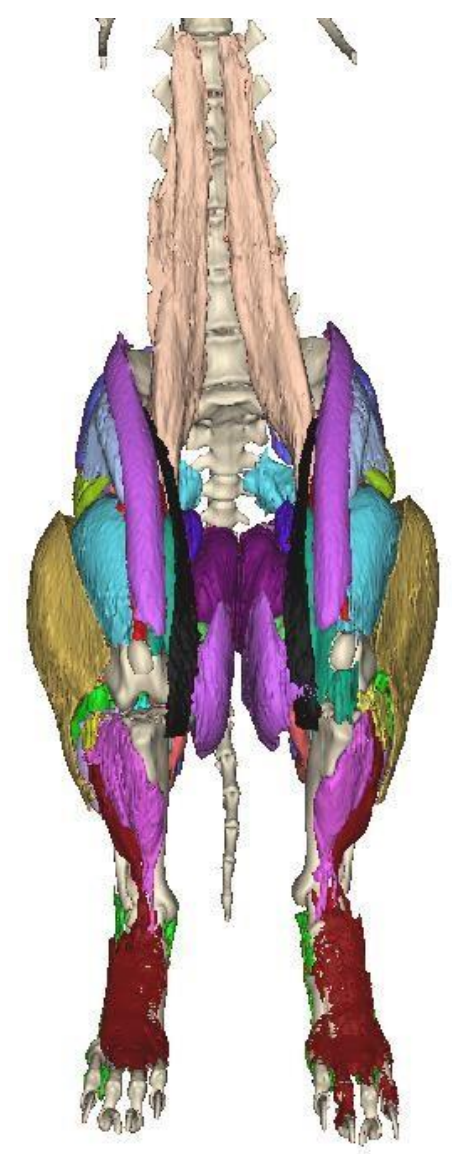

FIGURE 6 - Three-dimensional representation of bones and muscles of the pelvic limb (ventral). The lumbar and part of the thoracic spine were also included to visualize the attachment of the iliopsoas. 
April 12, 2016

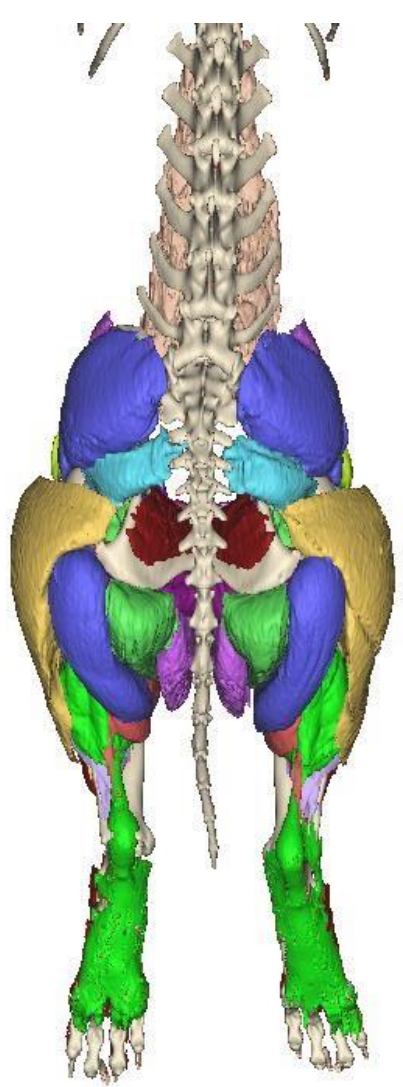

FIGURE 7 - Three dimensional representation of bones and muscles of the pelvic limb (dorsal).

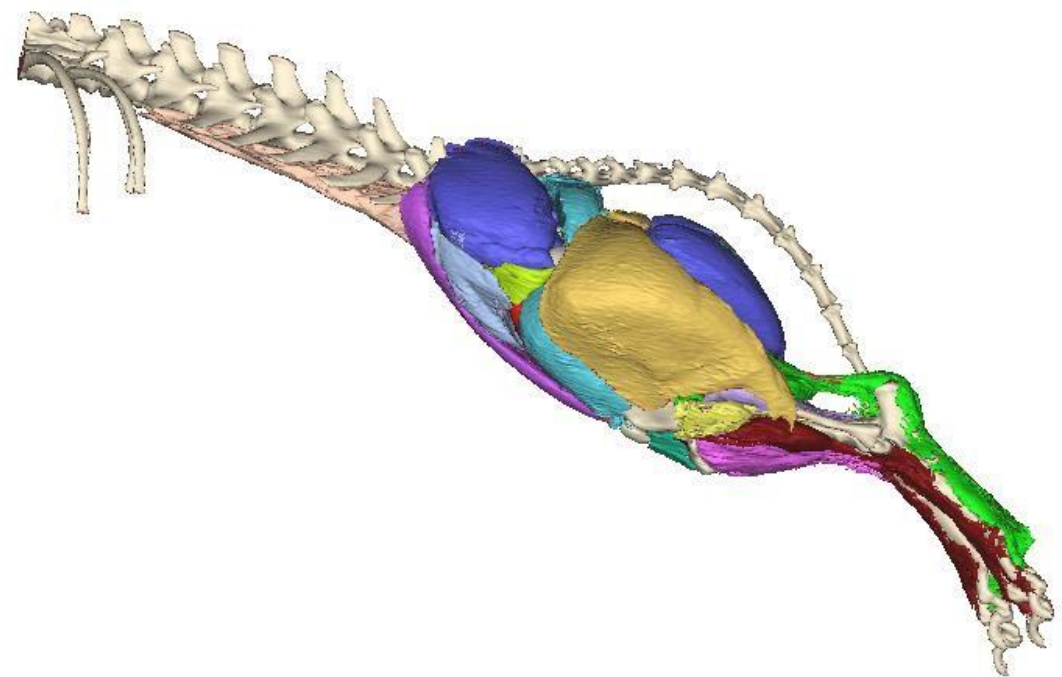

FIGURE 8 - Three dimensional representation of bones and muscles of the pelvic limb (sagittal, left side). 


\section{$\underline{\text { 1. Body Parameters }}$}

Each rigid body in OpenSim is defined by mass, center of gravity, moments of inertia, and joints and translations relative to a parent body.

Volume for trabecular/cortical bone, muscle, and fat was determined using medical imaging and processing software. ${ }^{a}$ Average densities for cortical bone $\left(2.003 \mathrm{~g} / \mathrm{cm}^{3}\right)$, trabecular bone $\left(1.911 \mathrm{~g} / \mathrm{cm}^{3}\right)$, muscle $\left(1.06 \mathrm{~g} / \mathrm{cm}^{3}\right)$, and fat $\left(0.95 \mathrm{~g} / \mathrm{cm}^{3}\right)$ were assumed based on available data on similar breeds of dogs (Gong et al., 1964; Ragetly et al., 2008). Mass for individual segments was calculated using

$$
m=\sum_{i=1}^{4} \rho_{i} P^{2} S N,
$$

where $i=1,2,3,4$ refers to cortical bone, trabecular bone, muscle, and fat; $\rho$ is the respective tissue density, $\mathrm{P}$ is the pixel width/height $(0.325 \mathrm{~mm}), \mathrm{S}$ is the slice thickness $(1 \mathrm{~mm})$, and $\mathrm{N}$ is the total number of pixels.

Center of gravity was calculated assuming a homogenous material. Bone axes of the pelvis, femur, tibia, tarsus, and phalanges were defined based on a canine study that adapts human definitions of bone axes set by the International Society of Biomechanics (Fu et al., 2010; Grood \& Suntay, 1983; Wu et al., 2002). Individual segment axes were defined in order to calculate joint angles between segments. The origin of the pelvis was set at the center of mass. The unit vector of the $\mathrm{x}$-axis $\left(\overrightarrow{x_{p}}\right)$ was defined using the midpoint of the left and right iliac crest (MPIC) and the midpoint of the left and right ischiatic tuberosity (MPIT) according to the following equation:

$$
\overrightarrow{x_{p}}=\frac{\vec{V}_{M P I C}-\vec{V}_{M P I T}}{\left|\vec{V}_{M P I C}-\vec{V}_{M P I T}\right|}
$$


The unit vector y-axis $\left(\overrightarrow{y_{p}}\right)$ was defined as the cross product of the vector from left to right iliac crest (LIC and RIC, respectively) and the x-axis according to the following equation:

$$
\overrightarrow{y_{p}}=\frac{\left(\vec{V}_{R I C}-\vec{V}_{L I C}\right) \times \overrightarrow{x_{p}}}{\left|\left(\vec{V}_{R I C}-\vec{V}_{L I C}\right) \times \overrightarrow{x_{p}}\right|}
$$

The remaining axis $\left(\overrightarrow{z_{p}}\right)$ was defined as the cross product of the $\mathrm{x}$ - and $\mathrm{y}$ - axes as follows:

$$
\overrightarrow{z_{p}}=\overrightarrow{x_{p}} \times \overrightarrow{y_{p}}
$$

The origin of the femur was set at the center of the femoral head. The unit vector $\mathrm{y}$-axis of the femur $\left(\overrightarrow{y_{f}}\right)$ was defined using the midpoint of the medial and lateral femoral condyles (MPFC) and the center of mass of the femur (FCOM), according to the following equation:

$$
\overrightarrow{y_{f}}=\frac{\vec{V}_{F C O M}-\vec{V}_{M P F C}}{\left|\vec{V}_{F C O M}-\vec{V}_{M P F C}\right|}
$$

The unit vector $\mathrm{x}$-axis of the femur $\left(\overrightarrow{x_{f}}\right)$ was defined as the cross product of the vector from medial and lateral femoral condyles (MFC and LFC, respectively) according to the following equation:

$$
\overrightarrow{x_{f}}=\frac{\overrightarrow{y_{f}} \times\left(\vec{V}_{L F C}-\vec{V}_{M F C}\right)}{\left|\overrightarrow{y_{f}} \times\left(\vec{V}_{L F C}-\vec{V}_{M F C}\right)\right|}
$$

The remaining axis $\left(\overrightarrow{z_{f}}\right)$ was defined as the cross product of the $\mathrm{x}$ - and $\mathrm{y}$ - axes as follows:

$$
\overrightarrow{z_{f}}=\overrightarrow{x_{f}} \times \overrightarrow{y_{f}}
$$


The origin of the tibia was set at the midpoint between the medial and lateral tibial condyle. The unit vector $y$-axis of the tibia $\left(\overrightarrow{y_{t}}\right)$ was defined using the midpoint of the medial and lateral malleolus (MPMal) and the center of mass of the tibia (TCOM), according to the following equation:

$$
\overrightarrow{y_{t}}=\frac{\vec{V}_{T C O M}-\vec{V}_{M P M a l}}{\left|\vec{V}_{T C O M}-\vec{V}_{M P M a l}\right|}
$$

The unit vector $\mathrm{x}$-axis of the tibia $\left(\overrightarrow{x_{t}}\right)$ was defined as the cross product of the vector from medial and lateral maleolus (MMal and LMal, respectively) according to the following equation:

$$
\overrightarrow{x_{t}}=\frac{\overrightarrow{y_{t}} \times\left(\vec{V}_{L M a l}-\vec{V}_{M M a l}\right)}{\left|\overrightarrow{y_{t}} \times\left(\vec{V}_{L M a l}-\vec{V}_{M M a l}\right)\right|}
$$

The remaining axis $\left(\overrightarrow{z_{t}}\right)$ was defined as the cross product of the $\mathrm{x}$ - and $\mathrm{y}$ - axes as follows:

$$
\overrightarrow{z_{t}}=\overrightarrow{x_{t}} \times \overrightarrow{y_{t}}
$$

The origin of the metatarsals was set at the midpoint of the lateral and medial malleolus. The unit vector y-axis of the metatarsals $\left(\overrightarrow{y_{m t}}\right)$ was defined using the midpoint of the medial and lateral malleolus (MPMal) and the center of mass of the metatarsals (MTCOM), according to the following equation:

$$
\overrightarrow{y_{m t}}=\frac{\vec{V}_{M T C O M}-\vec{V}_{M P M a l}}{\left|\vec{V}_{M T C O M}-\vec{V}_{M P M a l}\right|}
$$

The unit vector $\mathrm{x}$-axis of the metatarsals $\left(\overrightarrow{x_{m t}}\right)$ was defined as the cross product of the vector from medial and lateral malleolus (MMal and LMal, respectively) according to the following equation: 


$$
\overrightarrow{x_{m t}}=\frac{\overrightarrow{y_{m t}} \times\left(\vec{V}_{L M a l}-\vec{V}_{M M a l}\right)}{\left|\overrightarrow{y_{m t}} \times\left(\vec{V}_{L M a l}-\vec{V}_{M M a l}\right)\right|}
$$

The remaining axis $\left(\overrightarrow{z_{t}}\right)$ was defined as the cross product of the $\mathrm{x}$ - and $\mathrm{y}$ - axes as follows:

$$
\overrightarrow{z_{t}}=\overrightarrow{x_{t}} \times \overrightarrow{y_{t}}
$$

Joint centers were estimated based on previously mentioned bone geometries created from CT data. The center of rotation for the hip joint was defined by fitting a sphere to the surface of the femoral head. ${ }^{c}$ Hip center of rotation was then defined as the center of this sphere (Wu et al., 2002). The stifle joint was represented as a hinge with one degree of freedom. The center of rotation was assumed to be the midpoint between the contacts of the lateral femoral condyle/lateral tibial condyle and the medial femoral condyle/medial tibial condyle. Similarly, the tarsal joint was represented as a hinge with one degree of freedom. The center of rotation was assumed to be the midpoint between the contacts of the distal tibia and trochlea.

Each bony structure segmented within Mimics was individually exported as a stereolithography (STL) formatted file to create a 3D body. These files were used in OpenSim as representative geometry for visualization of the bony structures.

\section{Muscle Parameters}

Muscles included in the model are: biceps femoris, semitendinosus, semimembranosus, cranial/caudal sartorius, gracilis, pectineus, adductor magnus/brevis, tensor fascia lata, superficial gluteal, middle gluteal, deep gluteal, internal/external obturator, gemelli, quadratus femoris, rectus femoris, vastus medialis, vastus intermedius, vastus lateralis, iliopsoas, cranial tibial, long digital extensor, peroneus longus, 
gastrocnemius, superficial/deep digital flexors, and the popliteus. Individual muscles were segmented from the overall muscle mask based on a comprehensive guide of canine skeletal and muscle anatomy (Evans \& deLahunta, 1988).

Muscle volume and muscle centerline were determined for each individual muscle using medical imaging and processing software ${ }^{\mathrm{b}}$. Muscle volume was computed using the three dimensional reconstructions of each muscle. Muscle origin and insertion points were defined as the most proximal and distal points on the centerline and were visually confirmed using detailed anatomical guide of the dog (Evans \& deLahunta, 1988). Additionally, via points were added to describe wrapping around bone if necessary (based on visual inspection).

Additional muscle parameters needed are muscle fiber length and tendon slack length. Muscle fiber length was obtained from anatomic/morphometric studies of canine pelvic limbs (Shahar \& Milgram, 2001; Williams et al., 2008). Fiber lengths were directly modified using the following scaling factor

$$
\frac{f}{f_{m}}=\frac{l}{l_{m}}
$$

where $f$ is the desired fiber length, $f_{m}$ is the fiber length obtained from text, $l$ is the length of the bony segments that the individual muscle crosses, and $l_{m}$ is the length of the bony segments measured in the text. Tendon slack lengths were adjusted so that fiber lengths for each muscle operated within their optimal range during walking gait.

Muscle volume, fiber length, pennation angle, and specific tension were used to define the maximum isometric force for each muscle, using the following equation (Knarr et al., 2013): 


$$
F_{i s o}=\frac{\mathrm{V} * \sin (\alpha)}{l} * T
$$

Where $F_{\text {iso }}$ is maximum isometric force, $V$ is muscle volume, $\alpha$ is the pennation angle using values obtained from anatomic studies of dogs (Shahar \& Milgram, 2001; Williams et al., 2008), $l$ is scaled fiber length, and $T$ is muscle specific tension. Specific tension was estimated to be a constant $22.5 \mathrm{~N} / \mathrm{cm}^{2}$; this value is based on values of specific tension normalized to a sarcomere length of $2.2 \mu \mathrm{m}$ in various mammals (Brown et al., 1982; Powell et al., 1984; Roy et al., 1982; Spector et al., 1980).

\section{Kinematic Data}

\section{$\underline{1 . \text { Motion Capture System }}$}

All kinematic data was recorded at Texas A\&M University in the Veterinary Medicine and Biomedical Sciences Department using 10 Vicon T-Series infrared cameras recording at $100 \mathrm{~Hz}$ (Vicon Motion Systems, Denver, CO). Marker trajectory data was filtered using a fourth order Butterworth filter with a cutoff of $6 \mathrm{~Hz}$ before analysis. Camera position was optimized to capture the highest percentage of markers.

Additionally, sagittal plane video was captured at $100 \mathrm{~Hz}$ using digital Bonita cameras (Vicon Motion Systems, Denver, CO).

\section{Marker Set}

Motion was tracked using $9 \mathrm{~mm}$ diameter spherical reflective markers attached to the subject using double sided tape. Markers were placed over bony prominences to maintain 
consistency across trials and subjects. The locations of these markers are shown in Figure 9.

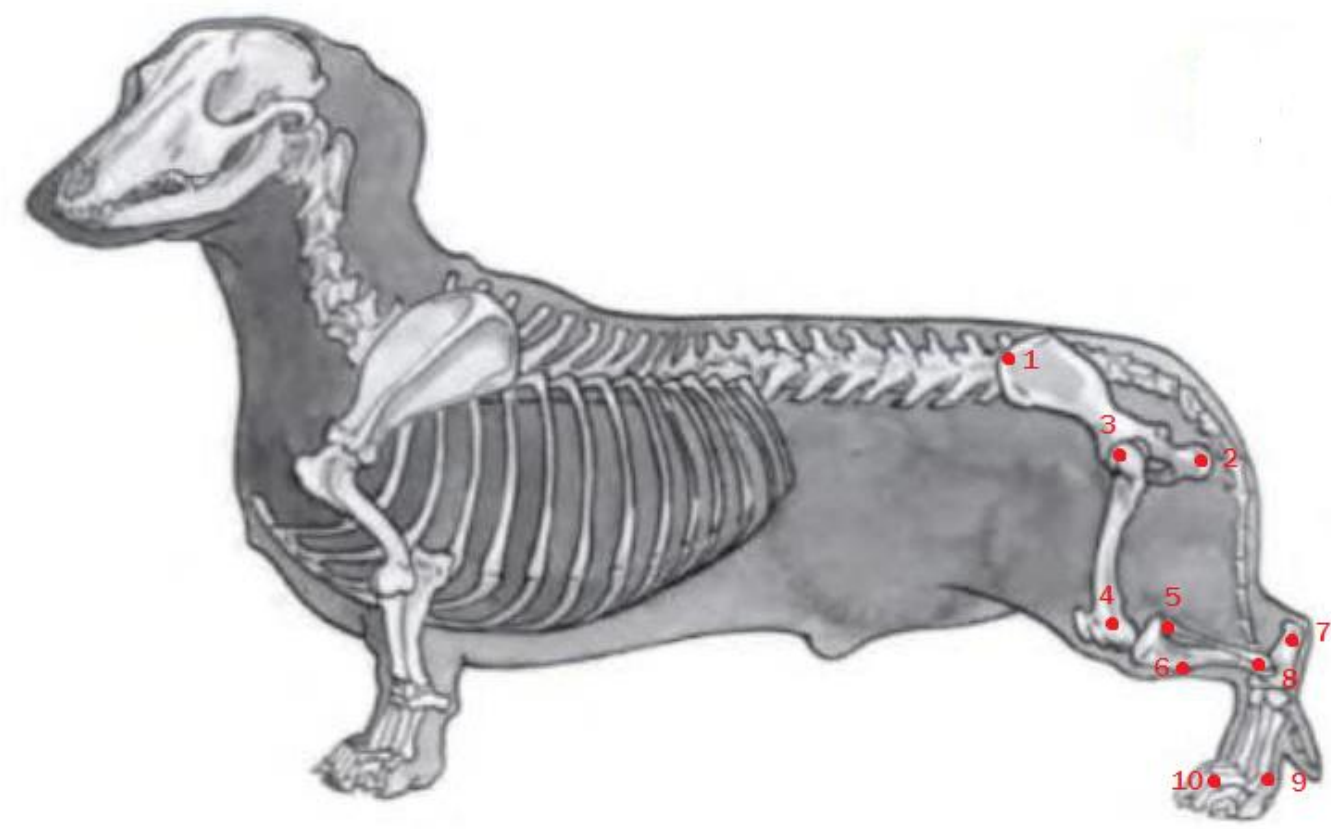

FIGURE 9 - Representative marker set used to acquire kinematic data; (1) cranial dorsal iliac spine (2) ischiatic tuberosity (3) greater trochanter (4) lateral femoral condyle (5) fibular head (6) tibial crest (7) calcaneus (8) lateral malleolus (9) distal aspect of the $5^{\text {th }}$ metatarsal (10) and the dorsal surface of the third pelvic limb digit. Image obtained from (Earle-Bridges, 2004).

\section{$\underline{\text { 3. Kinematic and Kinetic Data Collection }}$}

One kinematic data collection recording session consisted of 10 complete gait cycles, defined as both stance and swing phases for each limb. A successful trial was defined as a trial with only one paw striking a force plate at a given time and no marker gaps $\geq 5$ frames. Gaps in marker location data $<5$ frames due to marker obstruction were filled with spline functions. 
During each motion capture session, the dog stood in a static pose with all markers positioned on their body and visible to the motion capture cameras for 5-10 seconds. Following the static trial, medial markers (medial femoral condyle, medial malleolus, distal aspect of the $2^{\text {nd }}$ metatarsal, radial styloid process, and distal aspect of the $2^{\text {nd }}$ metacarpal) were removed. The static trial was used to adjust virtual marker locations in the model and scale the model in OpenSim.

Dynamic trials consisted of a dog walked by a handler across a force plate at a selfselected walking speed, with both kinematic and kinetic variables recorded. Dynamic trials were repeated as many times as possible without causing the subject discomfort or anxiety.

\section{Kinetic Data}

\section{Ground Reaction Force Kinetic Data}

Ground reaction forces (GRF) were measured and recorded at $1000 \mathrm{~Hz}$ using two AMTI OR-6 series force plates (AMTI Technologies, Newton, MA). Kinetic data was filtered using a fourth order Butterworth filter with a cutoff frequency of $50 \mathrm{~Hz}$. Force platform data collection was synchronized with the Vicon motion capture system to ensure that ground reaction forces could be timed properly in the OpenSim model.

During each trial the canine subject traversed the force platforms so that each pelvic limb paw contacted a unique force platform. Trials where multiple simultaneous paw contacts occurred on one force platform were discarded. It was necessary to accurately determine the magnitude, direction and application location of all external forces because 
unreliable measurements could lead to erroneous modeling results. Examples of data from a successful trial and a discarded trial are shown in Figures 10 and 11, respectively.

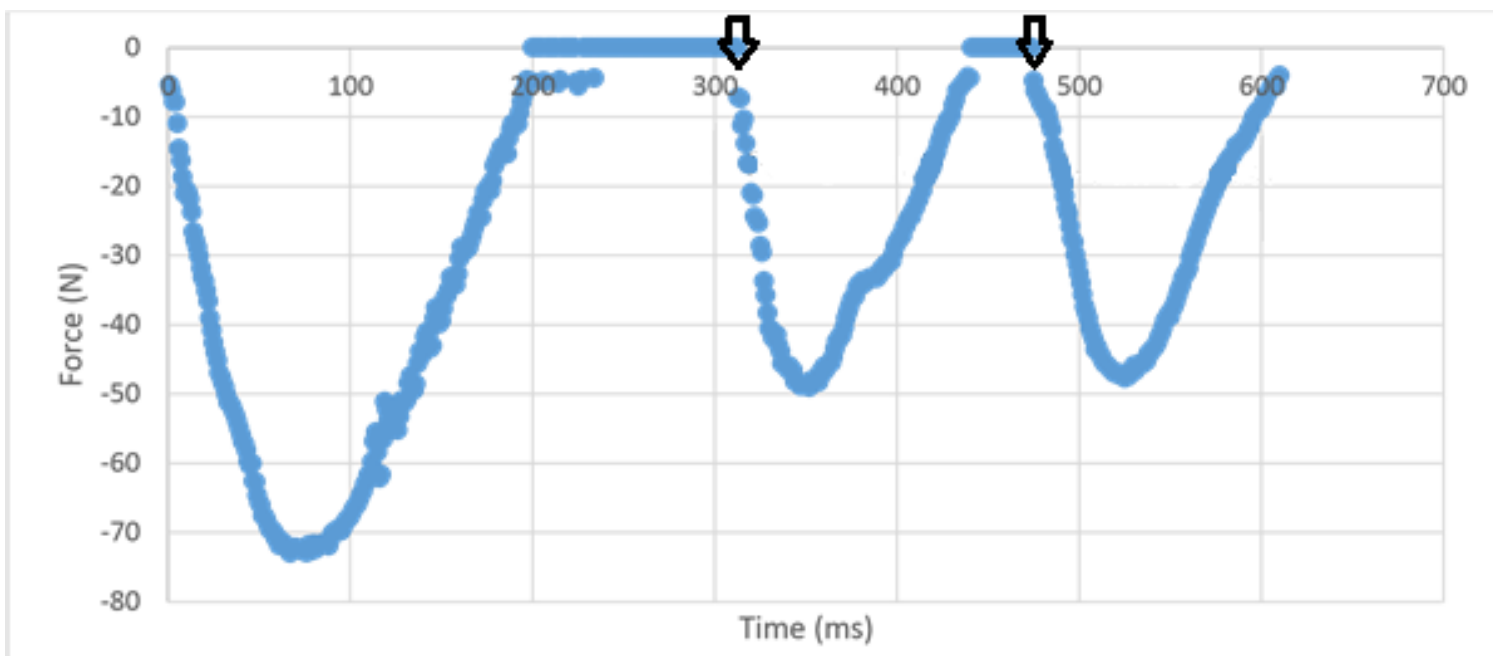

FIGURE 10 - Vertical ground reaction force time history for successful trial with only one paw in contact with force platform at any given time. Arrows indicate paw contact.

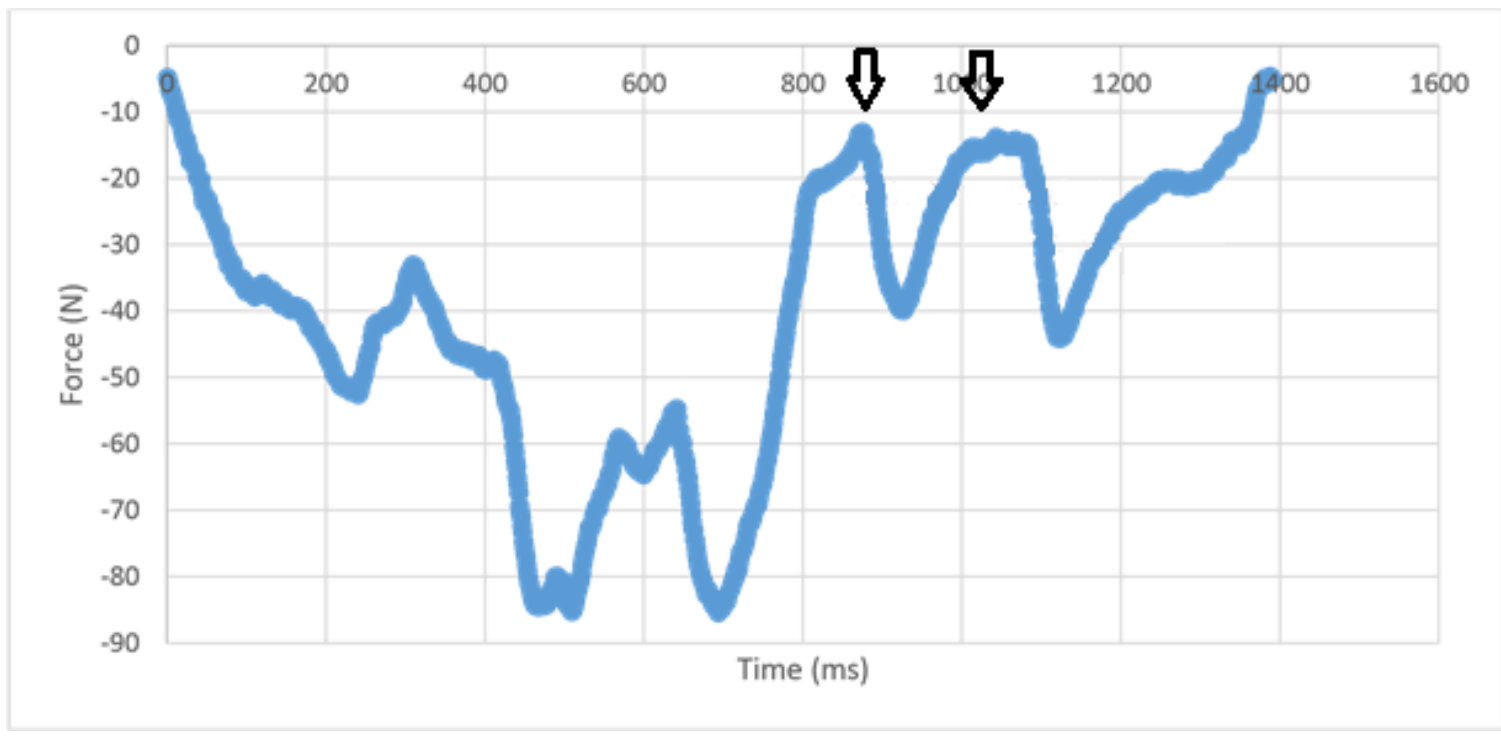

FIGURE 11 - Vertical ground reaction force time history for trial with multiple paws simultaneously in contact with the force platform. This figure illustrates the overlap between the right paw contact $(\sim 850 \mathrm{~ms})$ and left paw contact $(1000 \mathrm{~ms})$. 


\section{E. Incorporation of Kinematic and Kinetic Data into OpenSim}

Motion tracking data was applied to the model to replicate the desired motion. Ground reaction force data was then incorporated to adjust the mass distributions and joint kinematics of the model. Finally, joint torque was calculated within OpenSim followed by a prediction of muscle forces necessary to generate the joint moments. An overview of these steps is shown in Figure 12. Each step is described in detail in sections $1-3$

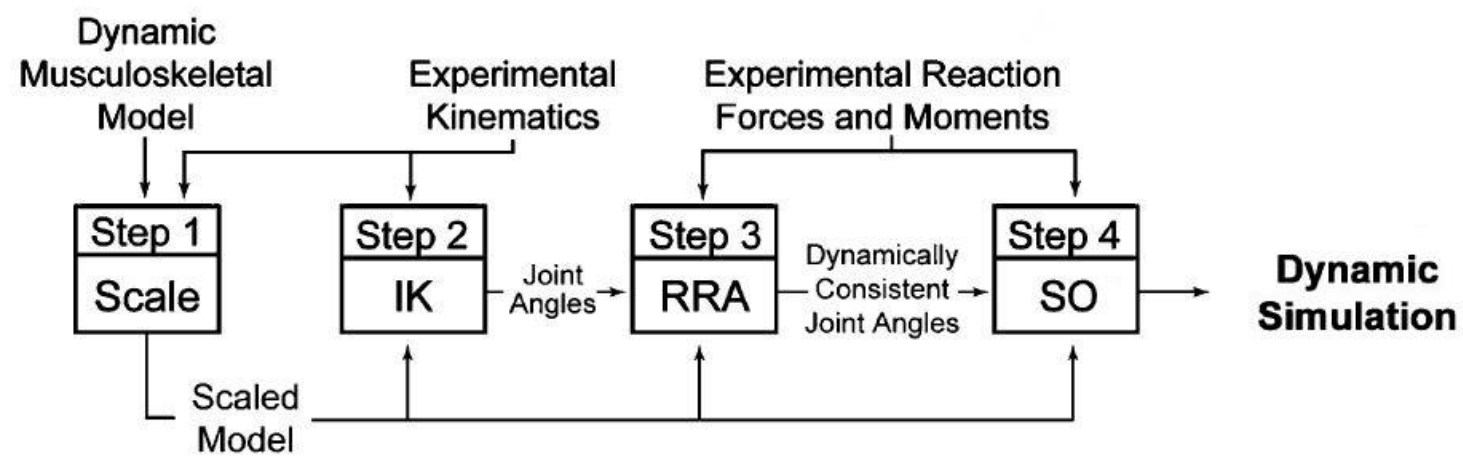

FIGURE 12 - An overview of the steps in creating a dynamic simulation in OpenSim, where IK is Inverse Kinematics, RRA is Residual Reduction Algorithm, and SO is Static Optimization. From (Delp et al., 2007).

\section{$\underline{1 . \text { Inverse Kinematics }}$}

Inverse kinematics was performed using the musculoskeletal model of the first healthy subject. Inputs and outputs of the inverse kinematics step are shown in Figure 13. 


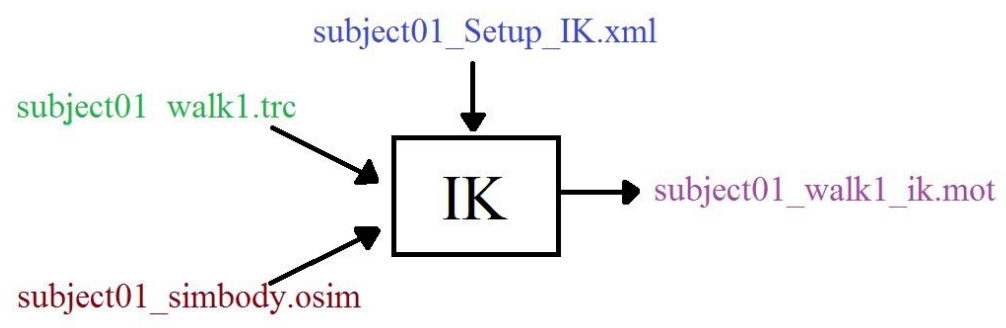

FIGURE 13 - Inputs and outputs of the inverse kinematics step, where 'subject01_simbody.osim' represents the musculoskeletal model, 'subject01_walk1.trc' represents the motion tracking data, and 'subject01_walk1_ik.mot' is the motion file created by inverse kinematics. 'subject01_Setup_IK.xml' is a setup file that can be created to bypass the need for manually entering setup values in the graphic user interface (Hicks 2012).

\section{Residual Reduction Algorithm}

The Residual Reduction Algorithm (RRA) process was conducted by combining joint angles from the Inverse Kinematics step defined in Section 3.4.1 with kinetic data to drive mass distribution and joint kinematics to be more consistent with external forces. This is accomplished by slightly altering body segment mass parameters to reduce the residual force.

Additionally, the residual forces and moments were applied to the pelvis-ground joint to represent the six degrees of freedom between the model and the ground (a body with fixed location and no connections to other bodies in the model). The output of this step is adjusted inverse kinematic data that includes joint angle time histories and segment motion time histories with respect to the ground segment. 


\section{Static Optimization}

Static Optimization was conducted using the adjusted kinematic data determined from the RRA process to calculate muscle forces necessary to create segment movement and joint torques. Static Optimization uses the adjusted kinematics and ground reaction forces determined from the Residual Reduction Algorithm to generate muscle activation and muscle force time histories. To accomplish this, the muscle forces required to maintain model equilibrium are calculated iteratively for each time step of the gait cycle. The calculated muscle forces are constrained by solving a least squares problem at each step (minimizing the sum of squared muscle activations required to maintain equilibrium).

\section{$\underline{\text { F. Analysis }}$}

To describe joint angles, coordinate systems of the pelvis, femur, tibia, and metatarsus were defined using marker locations. A three dimensional axis was defined for the pelvis. Axes for the femur, tibia, and metatarsus were defined using a single axis along the shaft of the bone due to the inability to capture medial markers during gait. Therefore, joint angles were only compared during flexion for this study.

The unit vector of the $\mathrm{x}$-axis of the pelvis $\left(\mathrm{x}_{\mathrm{p}}\right)$ was defined using the left ishiatic tuberosity (LIT) and left iliac crest (LIC) according to the following equation:

$$
\overrightarrow{x_{p}}=\frac{\vec{V}_{L I C}-\vec{V}_{L I T}}{\left|\vec{V}_{L I C}-\vec{V}_{L I T}\right|}
$$


The unit vector $\mathrm{y}$-axis $\left(y_{p}\right)$ was defined as the cross product of the vector from left to right ishiatic tuberosity (LIT and RIT, respectively) and the x-axis according to the following equation:

$$
\overrightarrow{y_{p}}=\frac{\left(\vec{V}_{R I T}-\vec{V}_{L I T}\right) \times \overrightarrow{x_{p}}}{\left|\left(\vec{V}_{R I T}-\vec{V}_{L I T}\right) \times \overrightarrow{x_{p}}\right|}
$$

The remaining axis $\left(z_{p}\right)$ was defined as the cross product of the $\mathrm{x}-$ and $\mathrm{y}$ - axes as follows:

$$
\overrightarrow{z_{p}}=\overrightarrow{x_{p}} \times \overrightarrow{y_{p}}
$$

The unit vector $y$-axis of the femur $\left(y_{f}\right)$ was defined using the greater trochanter (GT) and the lateral femoral condyle (LFC), according to the following equation:

$$
\overrightarrow{y_{f}}=\frac{\vec{V}_{G T}-\vec{V}_{L F C}}{\left|\vec{V}_{G T}-\vec{V}_{L F C}\right|}
$$

The unit vector y-axis of the tibia $\left(y_{t}\right)$ was defined using the tibial crest (TC) and lateral malleolus (LM), according to the following equation:

$$
\overrightarrow{y_{t}}=\frac{\vec{V}_{T C}-\vec{V}_{L M}}{\left|\vec{V}_{T C}-\vec{V}_{L M}\right|}
$$

The unit vector y-axis of the metatarsals $\left(y_{m t}\right)$ was defined using the calcaneus $(\mathrm{CAL})$ and distal $5^{\text {th }}$ metatarsal $(5 \mathrm{MT})$, according to the following equation: 


$$
\overrightarrow{y_{m t}}=\frac{\vec{V}_{C A L}-\vec{V}_{5 M T}}{\left|\vec{V}_{C A L}-\vec{V}_{5 M T}\right|}
$$

Hip flexion was defined as the angle formed between $y_{p}$ and $y_{f}$ around $z_{p}$. To accomplish this, first the projection of $y_{f}$ onto the plane formed by $x_{p}$ and $y_{p}$ was calculated as follows:

$$
\overrightarrow{y_{f(\text { pro })}}=\overrightarrow{y_{f}}-\overrightarrow{z_{p}}\left(\overrightarrow{y_{f}} \cdot \overrightarrow{z_{p}}\right)
$$

where $y_{f(p r o j)}$ is the projection of the femur axis. Hip flexion $(\alpha)$ was then calculated using the following equation:

$$
\alpha=\cos ^{-1}\left(\frac{\overrightarrow{y_{p}} \cdot \overrightarrow{y_{f(\text { pro })}}}{|| \overrightarrow{y_{p}} \| \cdot|| \overrightarrow{y_{f(\text { pro })}}||}\right)
$$

Stifle flexion $(\beta)$ was defined as the angle formed between $y_{f}$ and $y_{t}$, which was calculated using the following equation:

$$
\beta=\cos ^{-1}\left(\frac{\overrightarrow{y_{f}} \cdot \overrightarrow{y_{t}}}{|| \overrightarrow{y_{f}}|| \cdot\left|\overrightarrow{y_{t}}\right| \mid}\right)
$$

Tarsus flexion $(\gamma)$ was defined as the angle formed between $y_{t}$ and $y_{m t}$, which was calculated using the following equation:

$$
\gamma=\cos ^{-1}\left(\frac{\overrightarrow{y_{t}} \cdot \overrightarrow{y_{m t}}}{\left\|\overrightarrow{y_{t}}\right\| \cdot|| \overrightarrow{y_{m t}} \|}\right)
$$


A representative trial which included both stance and swing was used to compare marker trajectories, joint kinematics, and muscle activations. Healthy subject 1 was used to describe this representative trial, as CT data from this subject was used to build the model. It should be noted that the representative trial only includes data of the left limb; the right limb was discarded because of error in physical marker placement on the subject.

Joint angles were calculated from motion capture data using the definitions described above. Joint angles from the OpenSim model were originally exported directly from the inverse kinematics step. However, the definitions of bone axes in OpenSim differ from the definitions described using marker locations. Therefore, joint angles of the OpenSim model were calculated by exporting virtual marker trajectories, and calculating joint angles using the techniques described earlier in this section. Joint angles calculated using measured marker trajectories will be designated as "JCS", while joint angles calculated using virtual marker trajectories will be designated as "OS".

Five models were created for describing muscle activation (baseline, and 10\%, 20\%, $30 \%$, and $40 \%$ reduction in maximum isometric force). Muscle activations were exported directly from the static optimization step. Muscle activation and joint angle data were used to address the proposed hypotheses as follows:

Hypothesis 1: Modeled joint kinematics of the pelvic limb will not be different from kinematics calculated from marker trajectory data. 
Peak joint range of motion was calculated for the OS and JCS groups of a representative trial. Additionally, shape of curves was compared by calculating a correlation coefficient $(r)$ defined as:

$$
r=\frac{\sum_{i=1}^{n}\left(f_{i}-\bar{f}\right)\left(g_{i}-\bar{g}\right)}{\sqrt{\sum_{i=1}^{n}\left(f_{i}-\bar{f}\right)^{2} \sum_{i=1}^{n}\left(g_{i}-\bar{g}\right)^{2}}}
$$

Where $f_{i}$ and $g_{i}$ are the JCS and OS groups, respectively and $\bar{f}$ and $\bar{g}$ are the average angles. Validity range was assumed to be $r>0.80$ (Johansson et al., 2009).

Hypothesis 2: Measured motion tracking marker trajectories will not be different from virtual marker trajectories of the model.

Correlation coefficient given in Equation 34 was used to compare measured and virtual marker trajectories of relevant markers for a representative trial. Validity range was assumed to be $r>0.80$.

Hypothesis 3: Varying maximum isometric muscle force will affect peak muscle activation.

Model-predicted muscle activation patterns for Healthy Subject 1 were separated by gait phase (stance or swing) to evaluate the influence of maximum isometric muscle force on peak mulit-muscle activation. Peak muscle activation was calculated for each scenario across baseline, $-10 \%,-20 \%,-30 \%$ and $-40 \%$ maximum muscle isometric force. Sensitivity index $(S I)$ was used to describe the influence of the maximum isometric force parameter on peak muscle activation, defined as percentage change in output parameter (maximum muscle activation) divided by the percentage of change in input parameter 
(maximum isometric force). The $-10 \%,-20 \%,-30 \%$ and $-40 \%$ scenarios were all individually compared to baseline.

\section{RESULTS}

\section{A. Computed Tomography}

Muscle volumes, pennation angle (PA), scaled fiber length (FL), and maximum isometric force (max iso force) from the healthy dog are shown in Table VI.

\section{TABLE VI}

MUSCLE VOLUMES, PENNATION ANGLES, FIBER LENGTHS, AND MAXIMUM ISOMETRIC FORCES OF HEALTHY SUBJECT 1.

\begin{tabular}{|l|c|c|c|c|}
\hline \multicolumn{1}{|c|}{ Muscle } & $\begin{array}{c}\text { Volume } \\
\left(\mathbf{c m}^{\mathbf{3}} \mathbf{)}\right.\end{array}$ & $\begin{array}{c}\text { PA } \\
\left.\mathbf{(}^{\circ}\right)\end{array}$ & $\begin{array}{c}\text { Fiber Length } \\
(\mathbf{m})\end{array}$ & $\begin{array}{c}\text { Max iso force } \\
(\mathbf{N})\end{array}$ \\
\hline Adductor magnus & 28.84 & 9 & 0.0312 & 205.67 \\
\hline Biceps femoris & 27.36 & 0 & 0.0342 & 179.97 \\
\hline Cranial tibial & 3.66 & 20 & 0.0275 & 28.11 \\
\hline Deep digital extensor & 3.96 & 6 & 0.0277 & 31.99 \\
\hline Gastrocnemius & 5.81 & 30 & 0.0083 & 135.72 \\
\hline Gemellus & 0.68 & 0 & 0.0057 & 26.7 \\
\hline Iliopsoas & 14.11 & 0 & 0.0183 & 173.71 \\
\hline Middle gluteal & 17.54 & 30 & 0.0183 & 186.56 \\
\hline Quadratus femoris & 1.32 & 0 & 0.0137 & 21.61 \\
\hline Rectus femoris & 5.82 & 20 & 0.0134 & 92.11 \\
\hline Sartorius & 5.85 & 0 & 0.0922 & 14.28 \\
\hline Semimembranosus & 19.29 & 0 & 0.0517 & 84.03 \\
\hline Semitendinosus & 13.51 & 0 & 0.0502 & 60.55 \\
\hline Tensor fascia lata & 5.06 & 17 & 0.0274 & 39.76 \\
\hline Vastus lateralis and intermedius & 11.55 & 13 & 0.0575 & 44.04 \\
\hline Vastus medialis & 6.52 & 25 & 0.0154 & 86.23 \\
\hline
\end{tabular}


Visualization of bony structures within the OpenSim model were obtained from threedimensional reconstructions of bone obtained from CT data. Figure 14 shows the model as displayed in OpenSim.

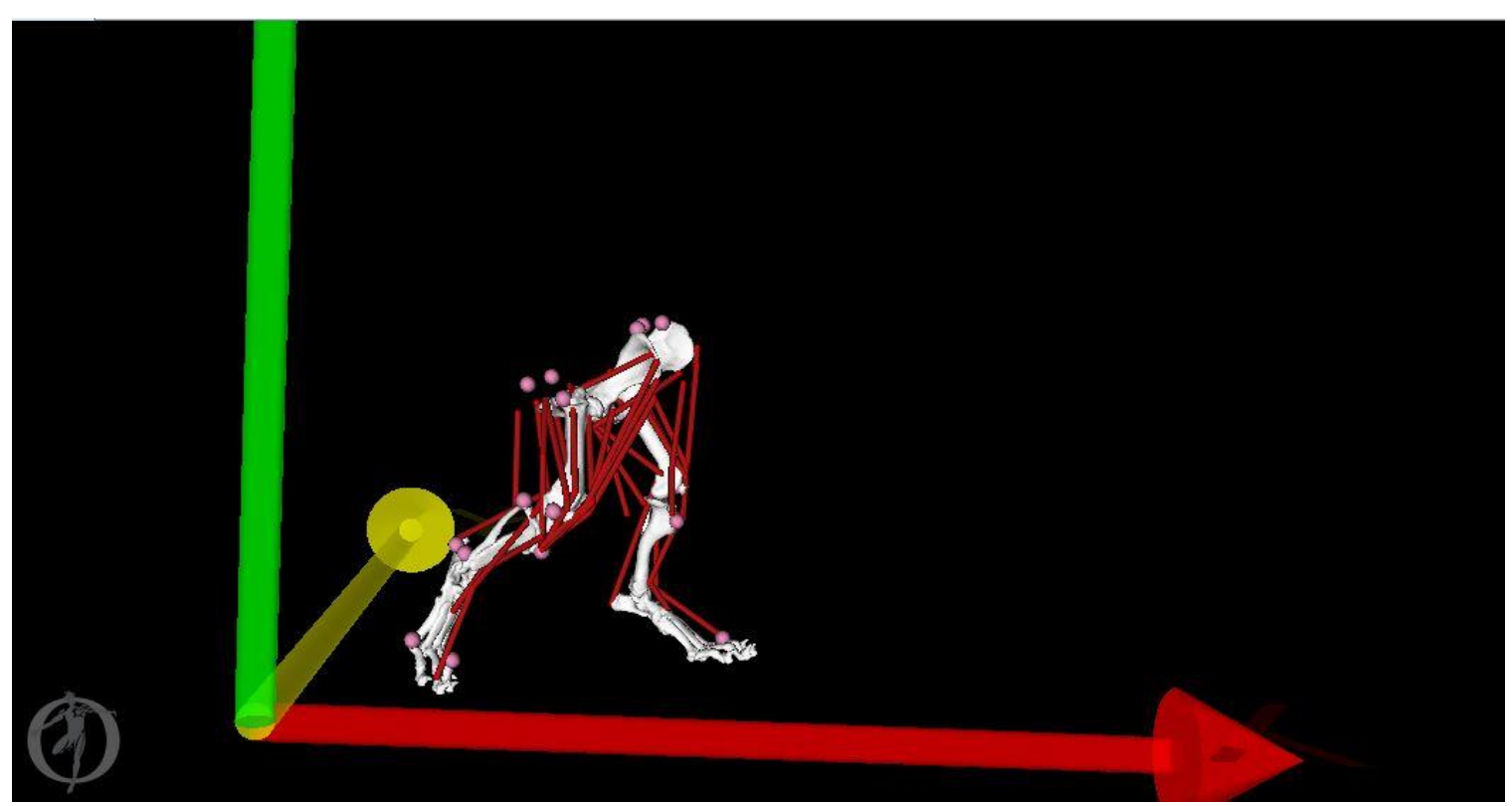

FIGURE 14 - Visualization of the OpenSim pelvic limb model. The model illustrates the boney segments, muscles and motion tracking markers. $\mathrm{X}$-axis is shown in red, $\mathrm{Y}$-axis is shown in yellow, and Z-axis is shown in green. The XY plane is representative of the ground, while gravity acts in the negative $\mathrm{Z}$ direction.

\section{B. Kinematic Data of Healthy Subjects}

The resulting joint angle curves (calculated using the JCS angles) for each healthy subject can be seen in Figures 15-17. Right limb joint angles were not included for healthy subject 1 due to marker placement error during recording. The numbers of trials used to create representative curves for each joint of all subjects are shown in Table VII.

\section{TABLE VII}


NUMBER OF TRIALS USED TO REPRESENT JOINTS DURING EACH PHASE OF GAIT.

\begin{tabular}{|c|c|c|c|}
\hline Healthy Subject 1 & $\begin{array}{c}\text { Hip } \\
\text { Angle }\end{array}$ & $\begin{array}{l}\text { Stifle } \\
\text { Angle }\end{array}$ & $\begin{array}{c}\text { Tarsus } \\
\text { Angle }\end{array}$ \\
\hline Left Stance & 10 & 10 & 10 \\
\hline Left Swing & 8 & 8 & 8 \\
\hline Right Stance & 8 & 8 & 8 \\
\hline Right Swing & 10 & 10 & 9 \\
\hline Healthy Subject 2 & $\begin{array}{c}\text { Hip } \\
\text { Angle }\end{array}$ & $\begin{array}{l}\text { Stifle } \\
\text { Angle }\end{array}$ & $\begin{array}{c}\text { Tarsus } \\
\text { Angle }\end{array}$ \\
\hline Left Stance & 9 & 10 & 10 \\
\hline Left Swing & 10 & 9 & 9 \\
\hline Right Stance & 10 & 8 & 8 \\
\hline Right Swing & 10 & 5 & 5 \\
\hline Healthy Subject 3 & $\begin{array}{c}\text { Hip } \\
\text { Angle }\end{array}$ & $\begin{array}{l}\text { Stifle } \\
\text { Angle }\end{array}$ & $\begin{array}{c}\text { Tarsus } \\
\text { Angle }\end{array}$ \\
\hline Left Stance & 10 & 10 & 9 \\
\hline Left Swing & 6 & 6 & 4 \\
\hline Right Stance & 8 & 9 & 9 \\
\hline Right Swing & 10 & 10 & 10 \\
\hline
\end{tabular}
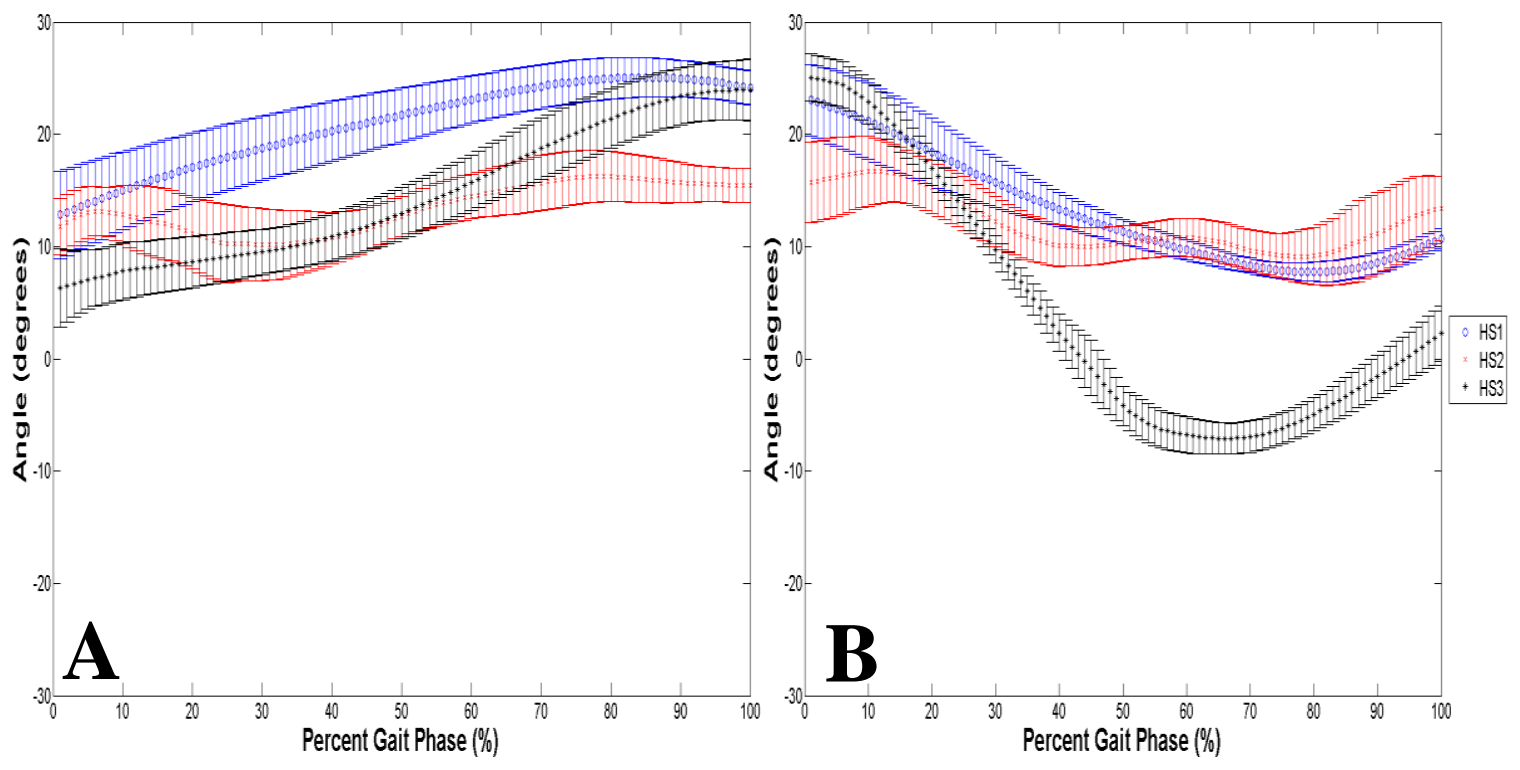

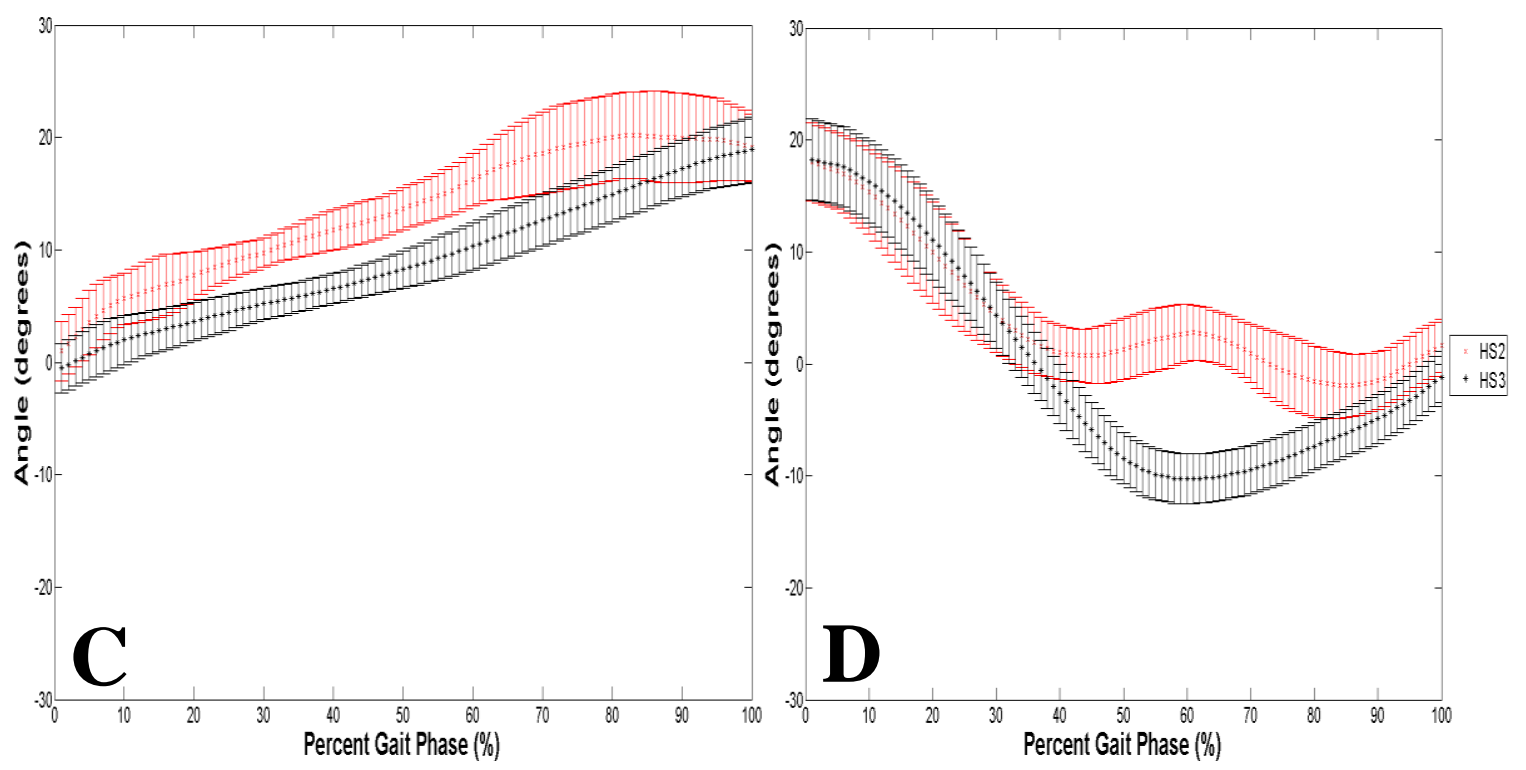

FIGURE 15 - Mean flexion/extension curves for the hip joint of individual subjects,

where HS1-3 are healthy subjects 1-3 respectively. A) is the left hip joint during stance

B) is the left hip joint during swing $\mathbf{C}$ ) is the right hip joint during stance and D) is the right hip joint during swing.
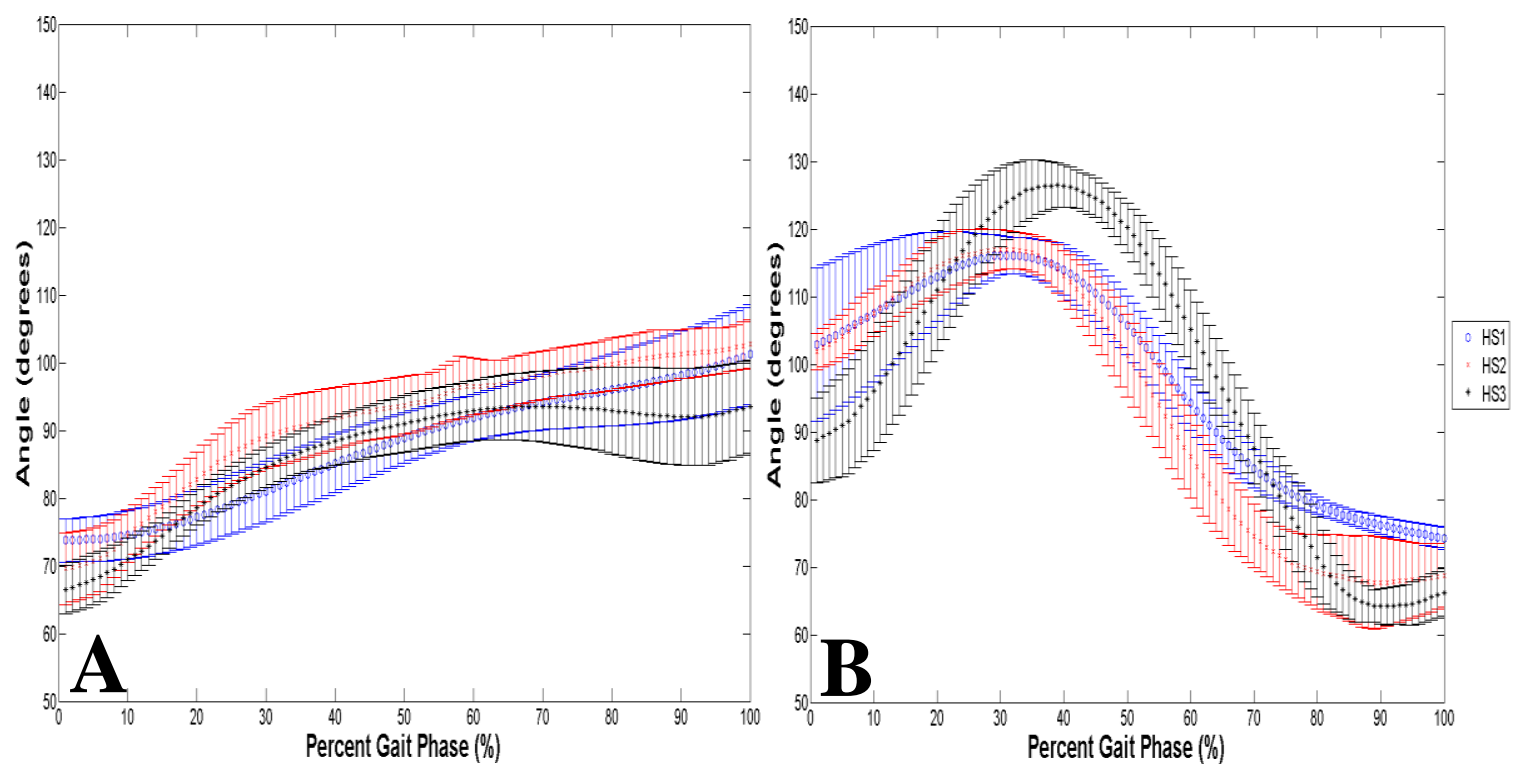

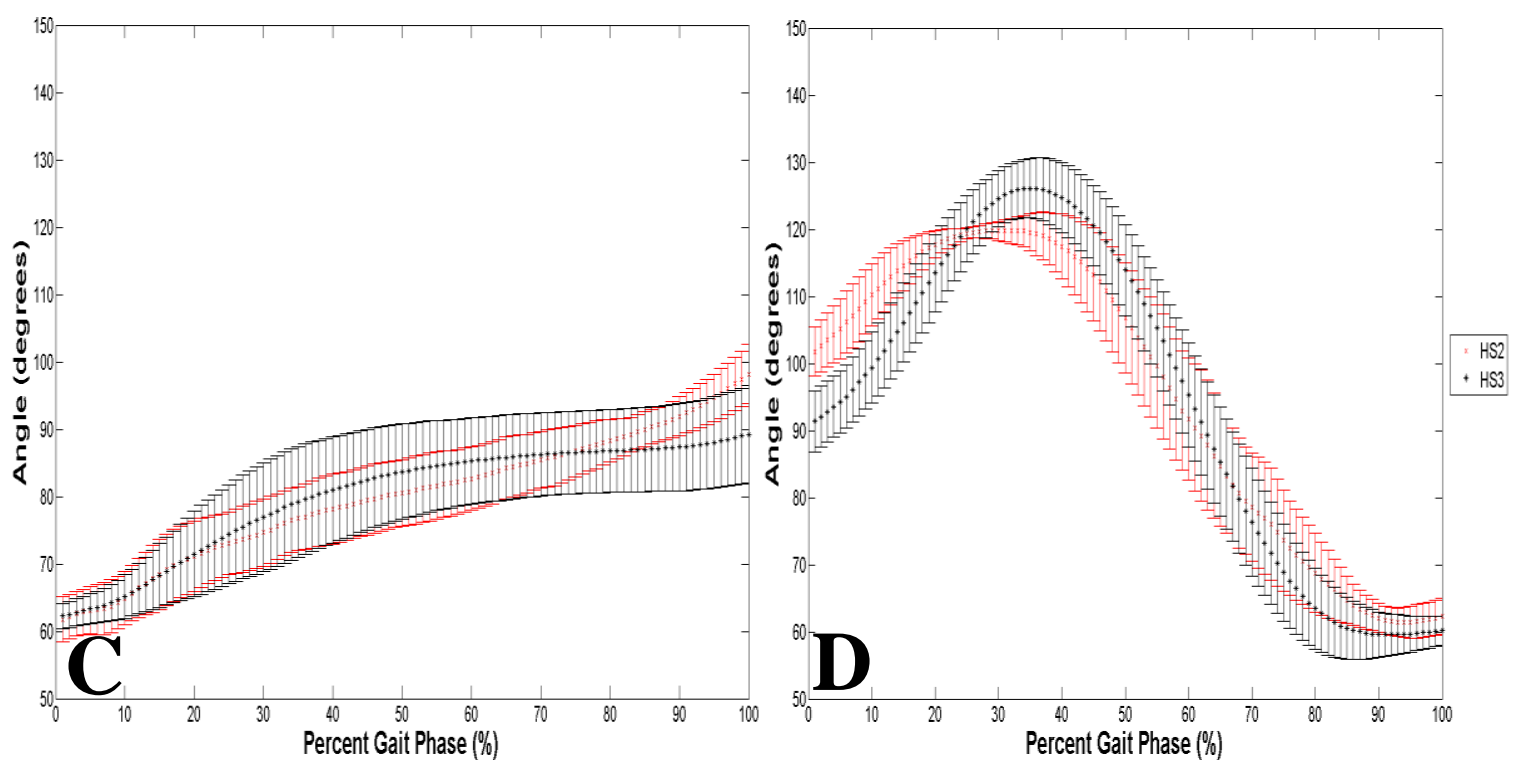

FIGURE 16 - Mean flexion/extension curves for the stifle joint of individual subjects,

where HS1-3 are healthy subjects 1-3 respectively. A) is the left stifle joint during stance

B) is the left stifle joint during swing $\mathbf{C}$ ) is the right stifle joint during stance and $\mathbf{D}$ ) is the right stifle joint during swing.
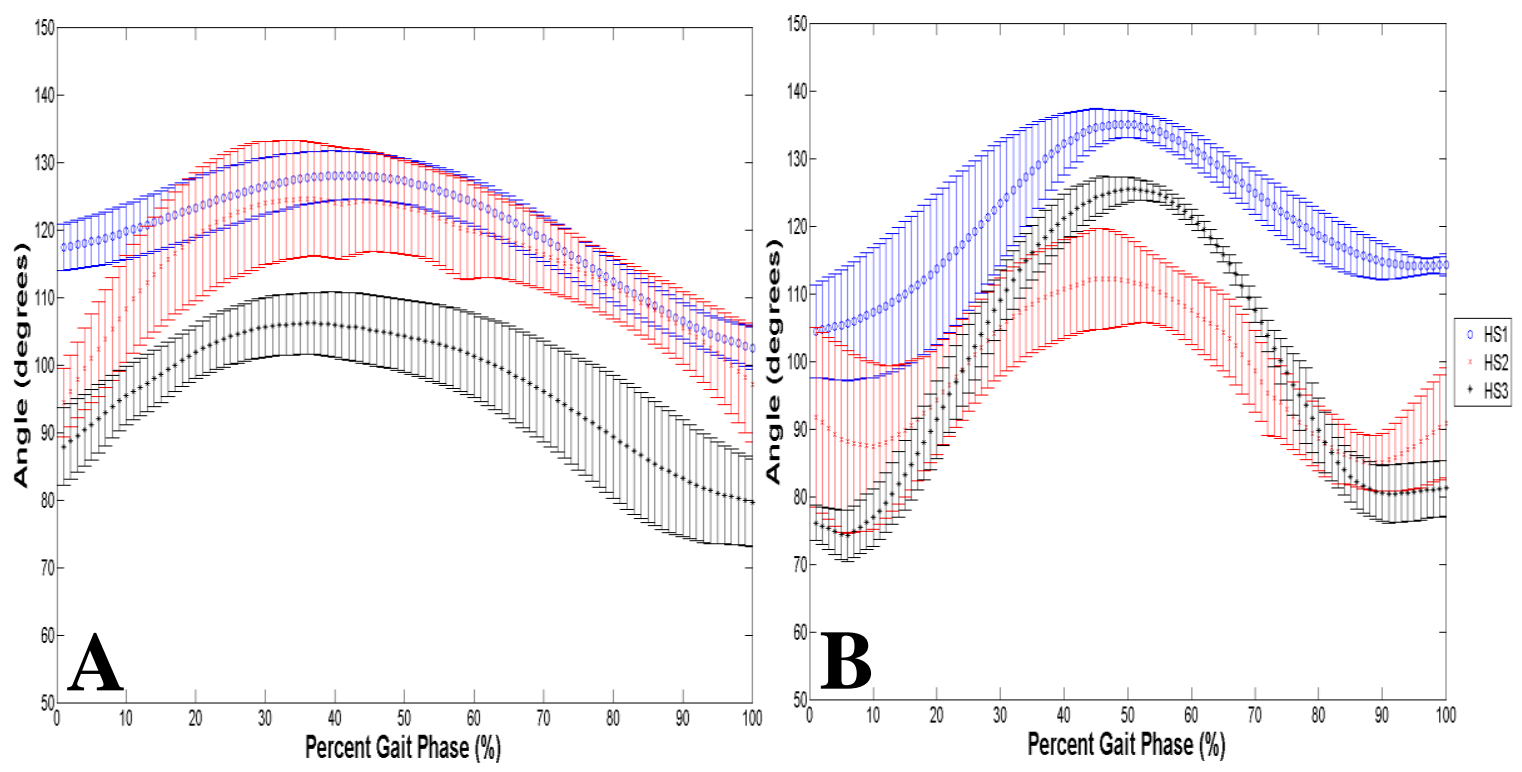

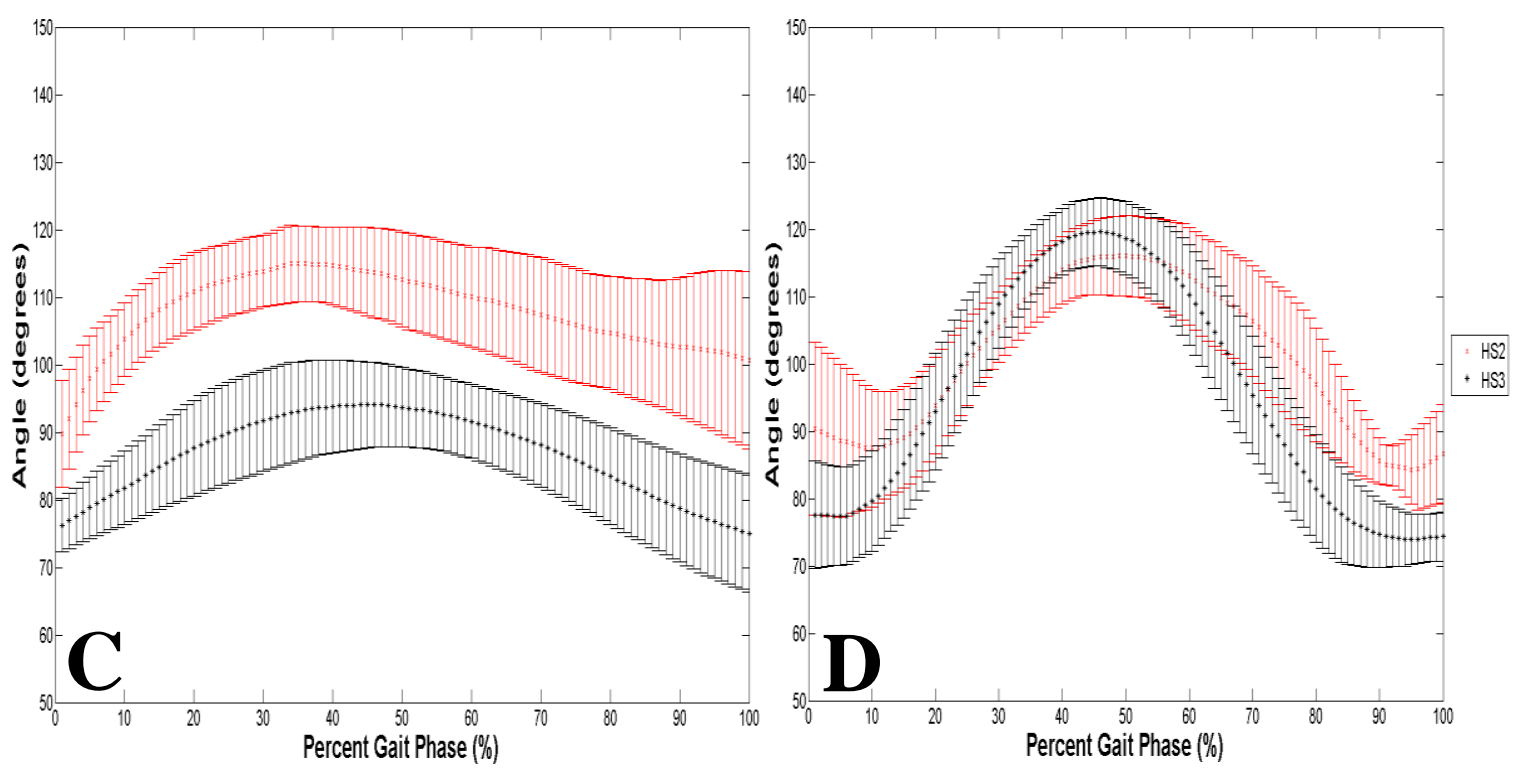

FIGURE 17 - Mean flexion/extension curves for the tarsus joint of individual subjects, where HS1-3 are healthy subjects 1-3 respectively. A) is the left tarsus joint during stance B) is the left tarsus joint during swing $\mathbf{C}$ ) is the right tarsus joint during stance and $\mathbf{D}$ ) is the right tarsus joint during swing.

Average speed of each subject is shown below in Table VIII.

TABLE VIII

AVERAGE WALKING SPEED OF HEALTHY SUBJECTS. VALUES WERE OBTAINED BY CALCULATING THE AVERAGE HORIZONTAL SPEED OF A MARKER LOCATED ON THE LUMBOSACRAL JUNCTION.

\begin{tabular}{|l|c|c|}
\hline Subject Name & Average speed (m/s) & n \\
\hline Healthy Subject 1 & $0.81 \pm 0.20$ & 14 \\
\hline Healthy Subject 2 & $0.57 \pm 0.16$ & 15 \\
\hline Healthy Subject 3 & $1.14 \pm 0.20$ & 19 \\
\hline
\end{tabular}

Walking speeds of healthy subjects were compared using a Wilcoxon rank-sum test. Significant differences were found between walking speeds of all 3 subjects (Healthy 
Subject 1-Healthy Subject $2 \mathrm{p}=0.0018$, Healthy Subject 1-Healthy Subject $2 \mathrm{p}<0.001$, Healthy Subject 2-Healthy Subject 3 p<0.001).

\section{Model Kinematic Reconstruction Comparison to Motion Capture}

Figures 18-20 show comparisons between the JCS and OS methods of the hip, stifle, and tarsus joint angles for healthy subject 1, left pelvic limb.

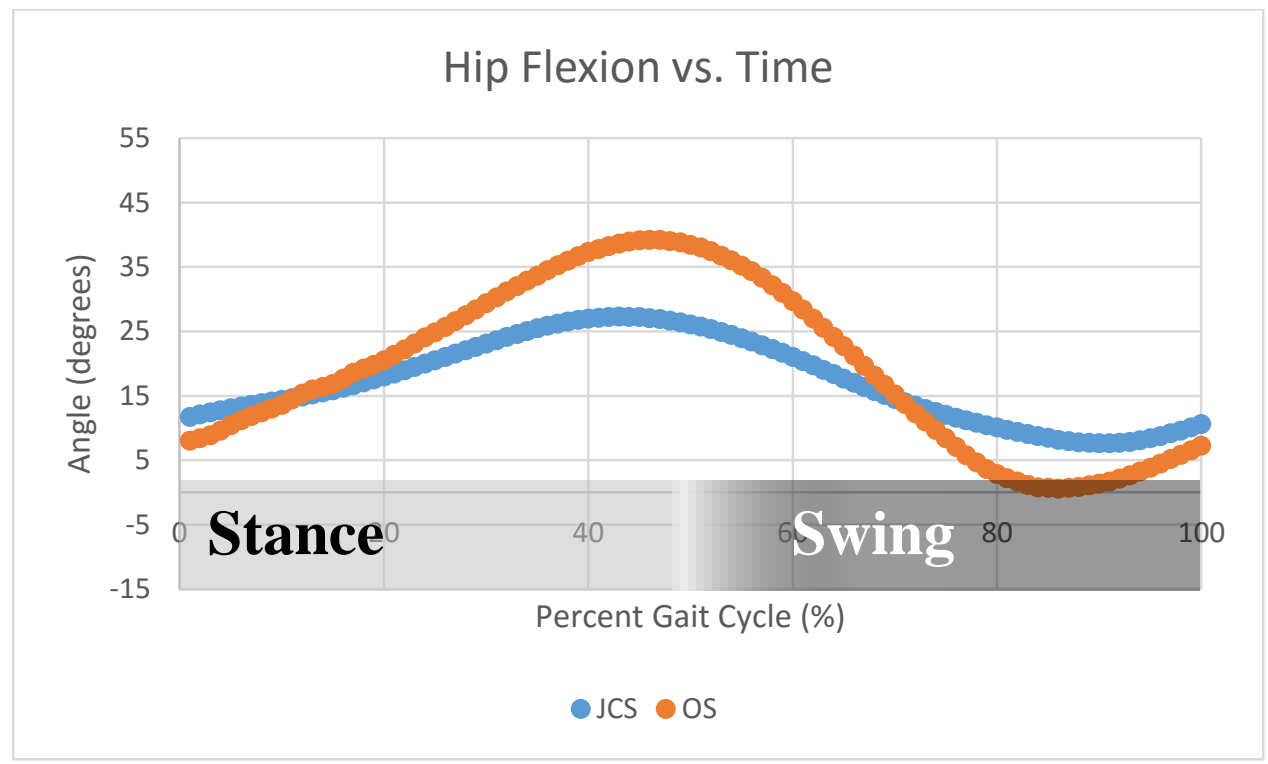

FIGURE 18 - Hip flexion of a representative trial using JCS and OS methods. Peak JCS angle of $27.3^{\circ}$ occurs at $43 \%$ gait, while peak OS angle of $39.3^{\circ}$ occurs at $47 \%$ gait. Correlation coefficient is 0.99 . 


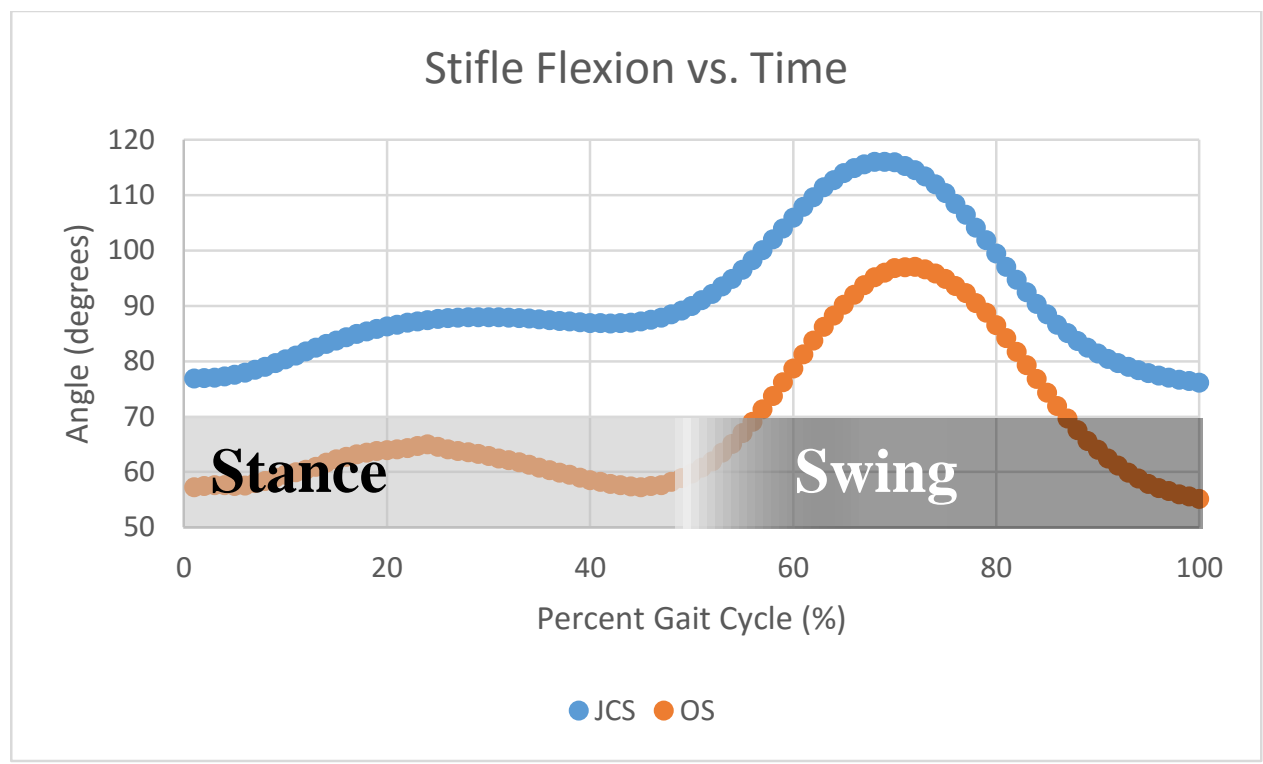

FIGURE 19 - Stifle flexion of a representative trial using JCS and OS methods. Peak JCS angle of $116.0^{\circ}$ occurs at $68 \%$ gait, while peak OS angle of $97.1^{\circ}$ occurs at $72 \%$ gait.

Correlation coefficient is 0.92 .

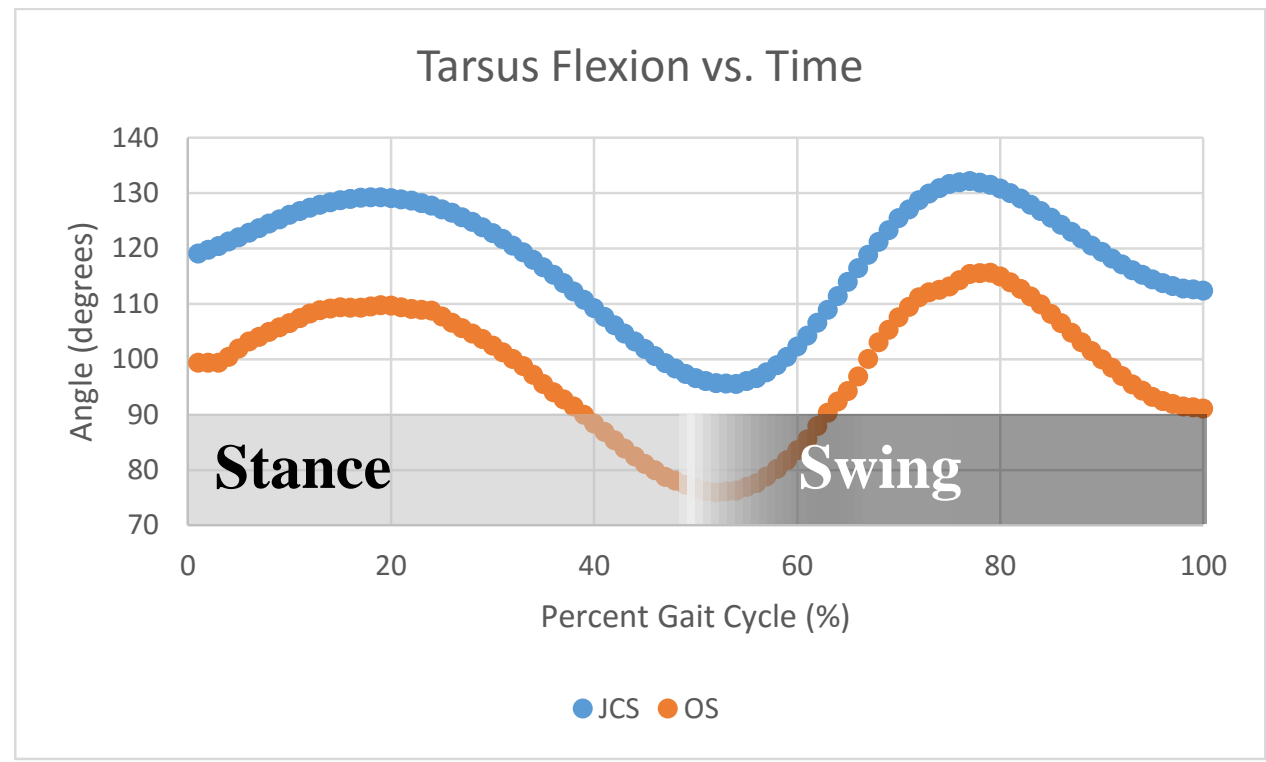

FIGURE 20 -_Tarsus flexion of a representative trial using JCS and OS methods. Peak JCS angle of $132.2^{\circ}$ occurs at $77 \%$ gait, while peak OS angle of $115.7^{\circ}$ occurs at $79 \%$ gait. Correlation coefficient is 0.99 . 


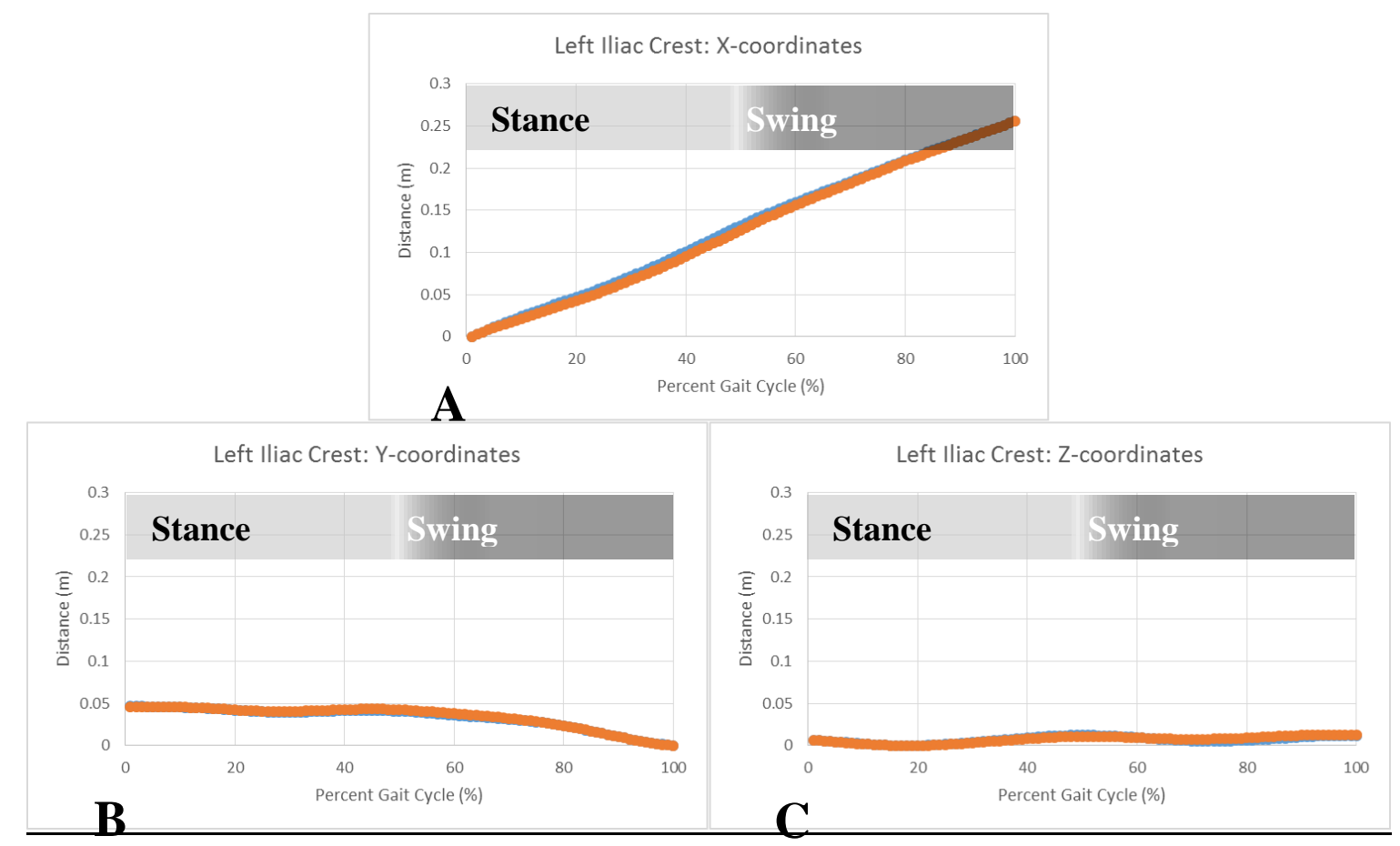

FIGURE 21 - Marker trajectory of the iliac crest in the A) cranial-caudal (x-direction) B) medial-lateral (y-direction) and C) ventral-dorsal (z-direction) directions. JCS marker trajectories are shown in blue, while OS marker trajectories are shown in orange. Correlation coefficients were $0.99,0.99$, and 0.93 in the $\mathrm{X}, \mathrm{Y}$, and Z directions respectively. The maximum total distance between JCS and OS marker locations was $0.64 \mathrm{~cm}$. 

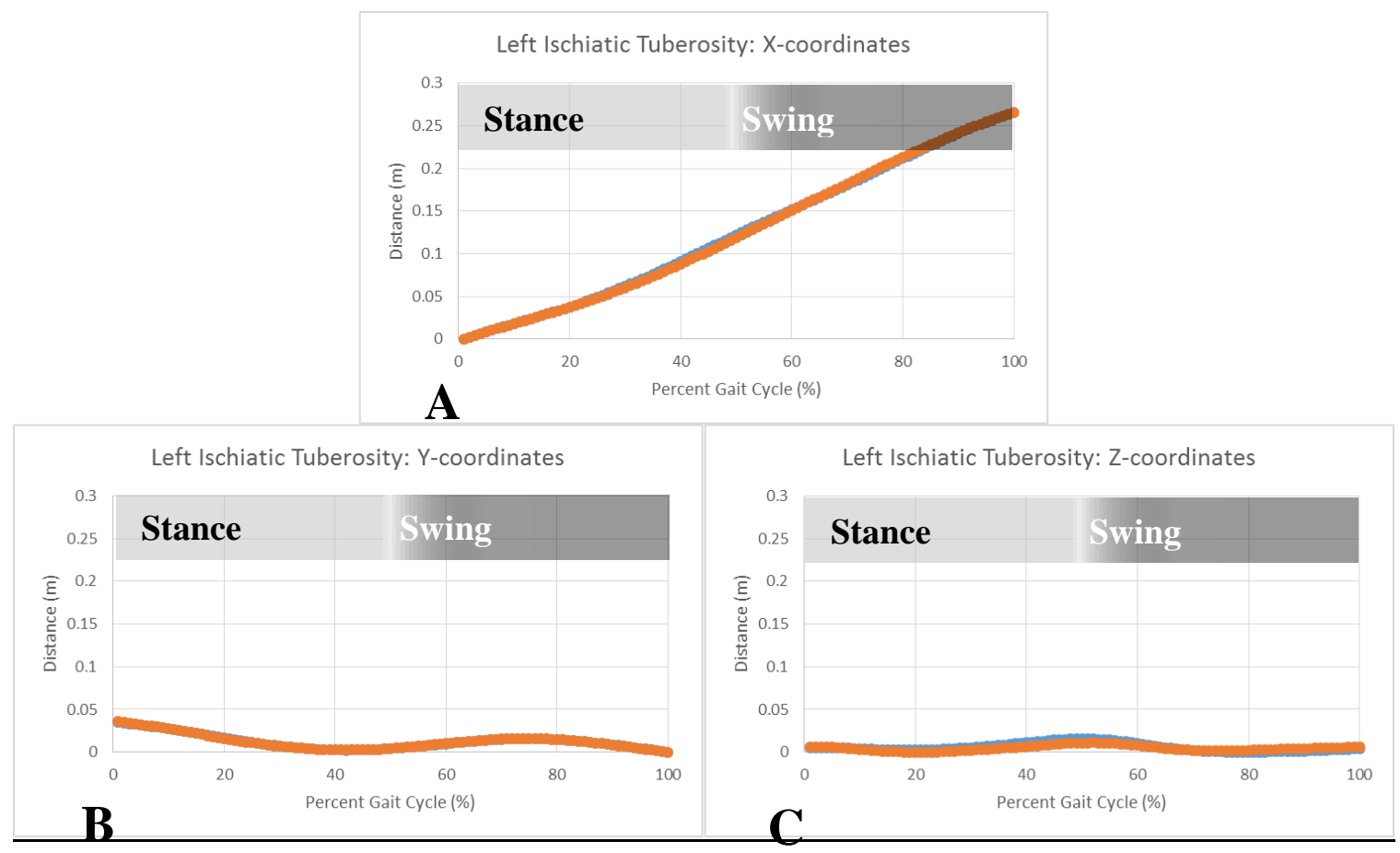

FIGURE 22 - Marker displacement of the ischiatic tuberosity in the A) cranial-caudal B) medial-lateral and C) vertical directions. JCS marker trajectories are shown in blue, while OS marker trajectories are shown in orange. Correlation coefficients were $0.99,0.99$, and 0.85 in the $\mathrm{X}, \mathrm{Y}$, and $\mathrm{Z}$ directions respectively. The maximum total distance between JCS and OS marker locations was $0.62 \mathrm{~cm}$. 

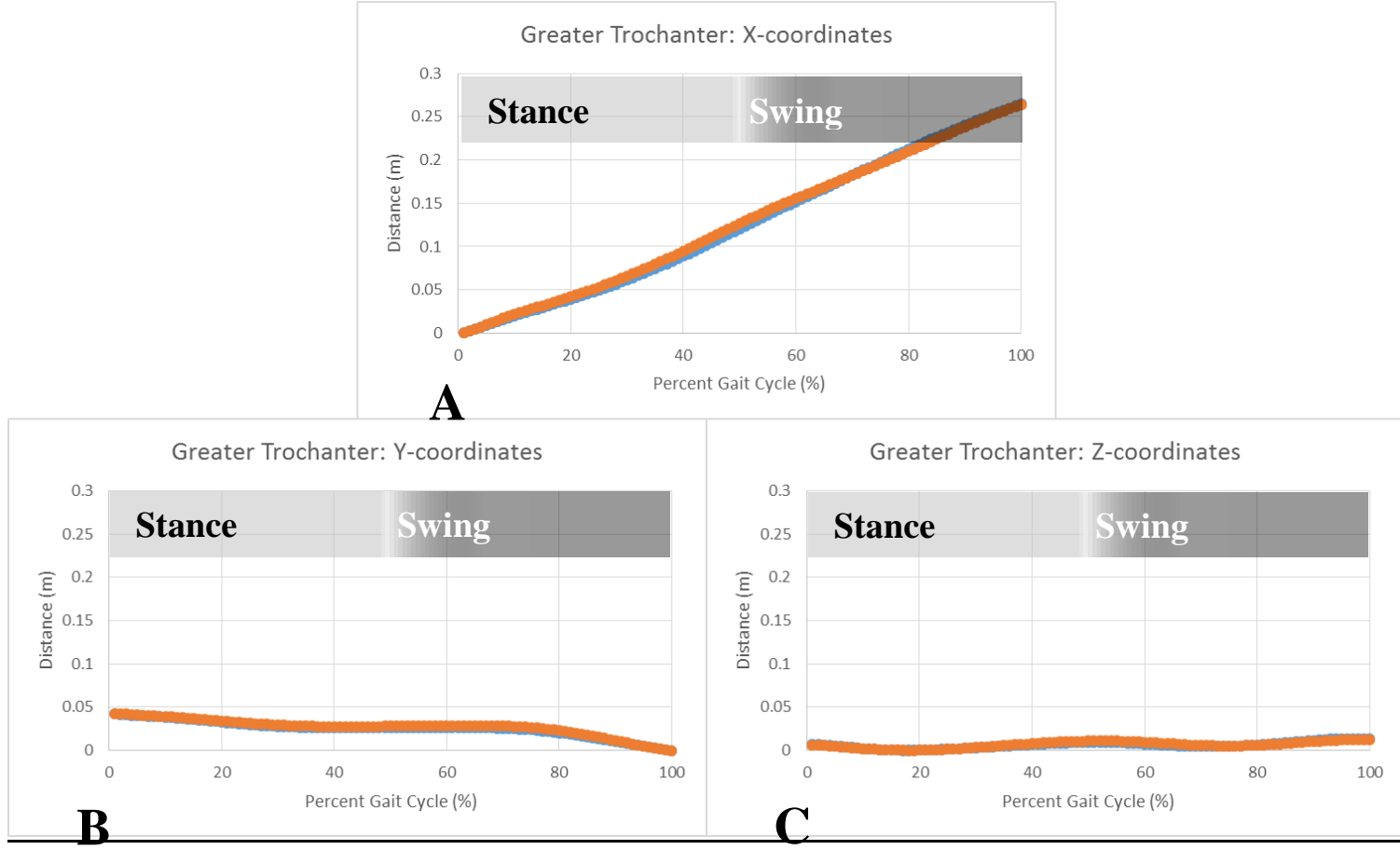

FIGURE 23 - Marker displacement of the greater trochanter in the A) cranial-caudal B) medial-lateral and C) vertical directions. JCS marker trajectories are shown in blue, while OS marker trajectories are shown in orange. Correlation coefficients were 0.99, 0.99, and 0.95 in the $\mathrm{X}, \mathrm{Y}$, and $\mathrm{Z}$ directions respectively. The maximum total distance between JCS and OS marker locations was $0.67 \mathrm{~cm}$. 


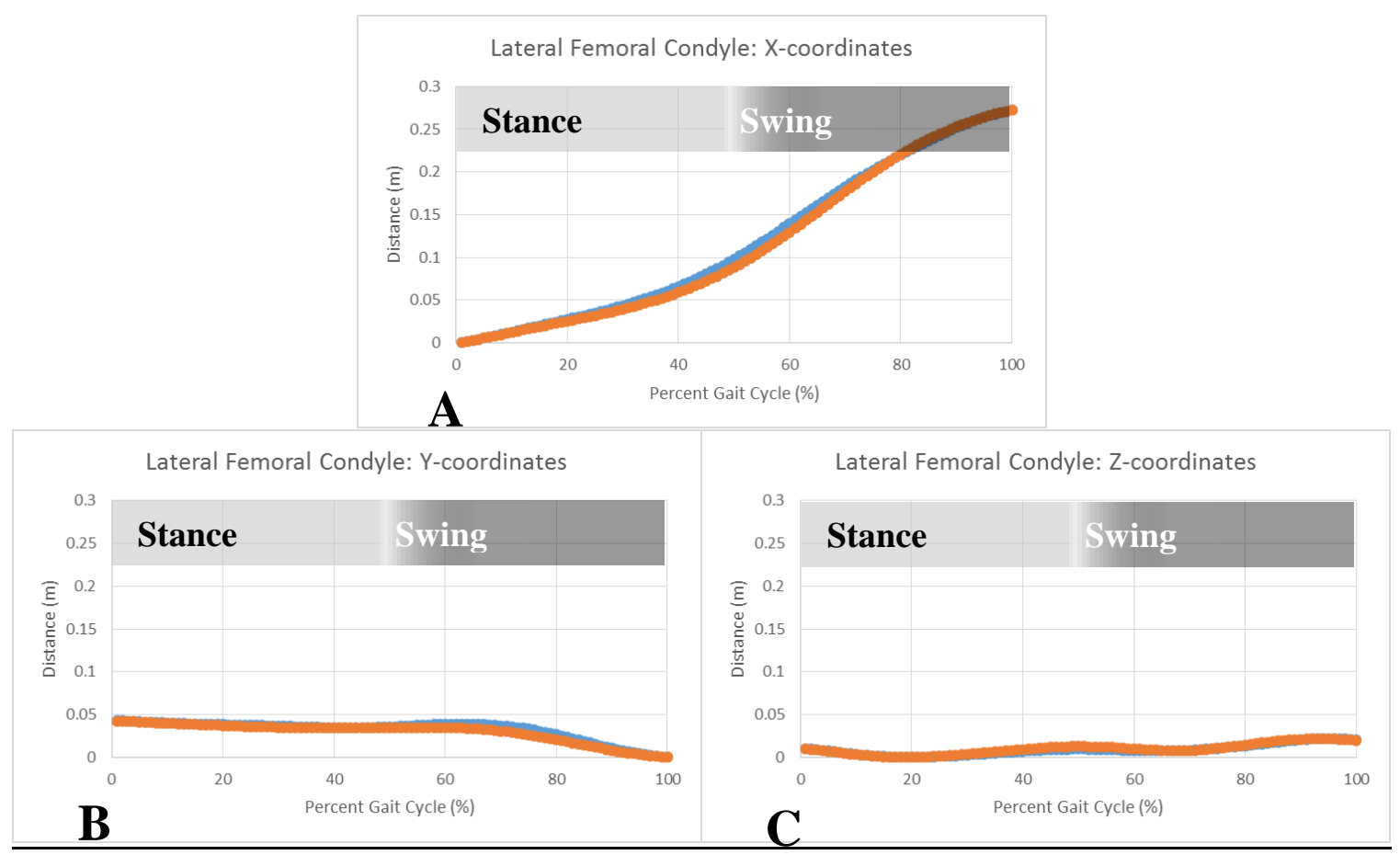

FIGURE 24 - Marker displacement of the lateral femoral condyle in the A) cranial-caudal B) medial-lateral and C) vertical directions. JCS marker trajectories are shown in blue, while OS marker trajectories are shown in orange. Correlation coefficients were 0.99 , 0.98, and 0.98 in the $\mathrm{X}, \mathrm{Y}$, and $\mathrm{Z}$ directions respectively. The maximum total distance between JCS and OS marker locations was $1.1 \mathrm{~cm}$. 


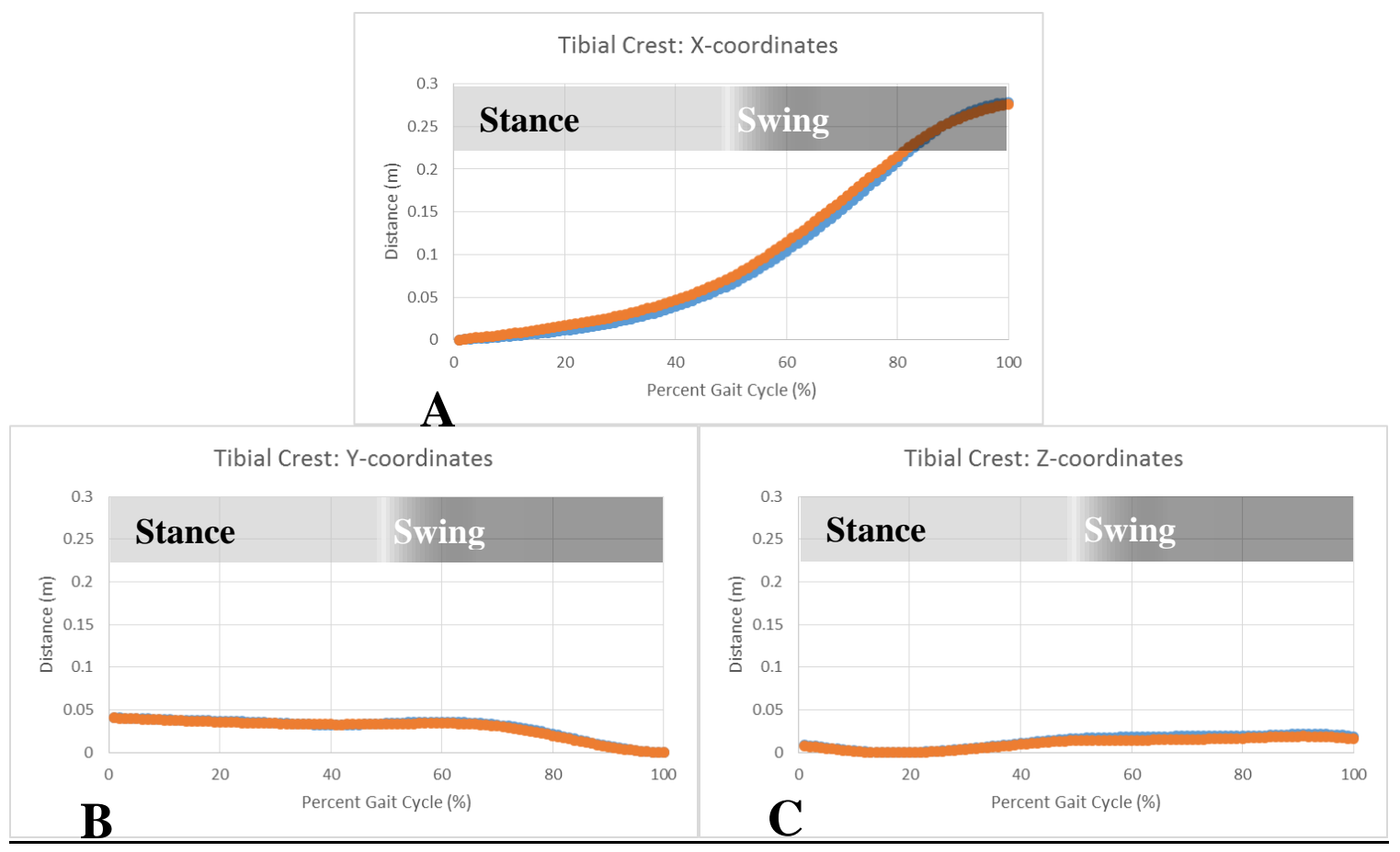

FIGURE 25 - Marker displacement of the tibial crest in the A) cranial-caudal B) mediallateral and C) vertical directions. JCS marker trajectories are shown in blue, while OS marker trajectories are shown in orange. Correlation coefficients were 0.99, 0.99, and 0.99 in the $\mathrm{X}, \mathrm{Y}$, and $\mathrm{Z}$ directions respectively. The maximum total distance between JCS and OS marker locations was $1.2 \mathrm{~cm}$. 


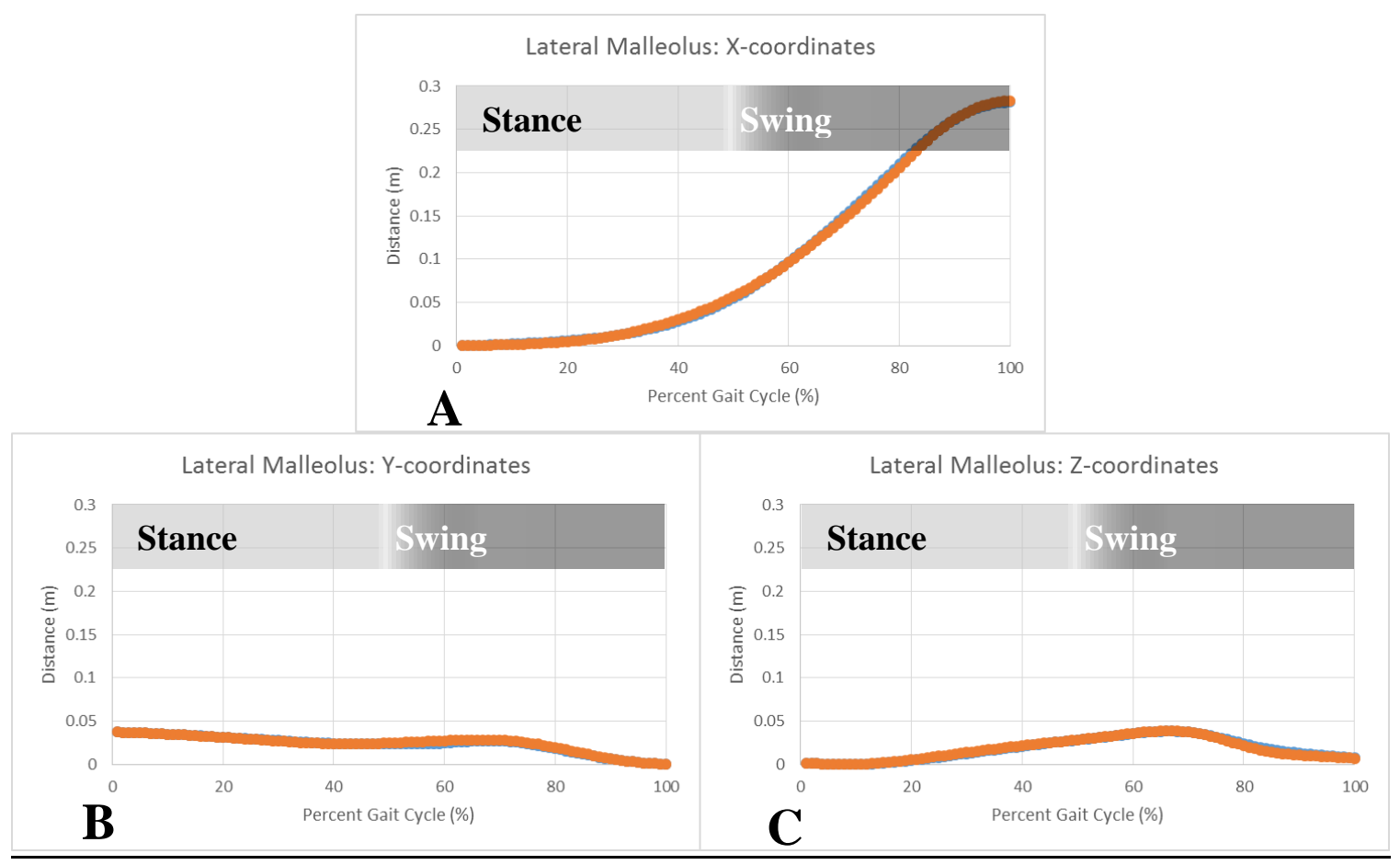

FIGURE 26 - Marker displacement of the lateral malleolus in the A) cranial-caudal B) medial-lateral and C) vertical directions. JCS marker trajectories are shown in blue, while OS marker trajectories are shown in orange. Correlation coefficients were 0.99, 0.99, and 0.99 in the $\mathrm{X}, \mathrm{Y}$, and $\mathrm{Z}$ directions respectively. The maximum total distance between JCS and OS marker locations was $\mathrm{s} 0.47 \mathrm{~cm}$. 


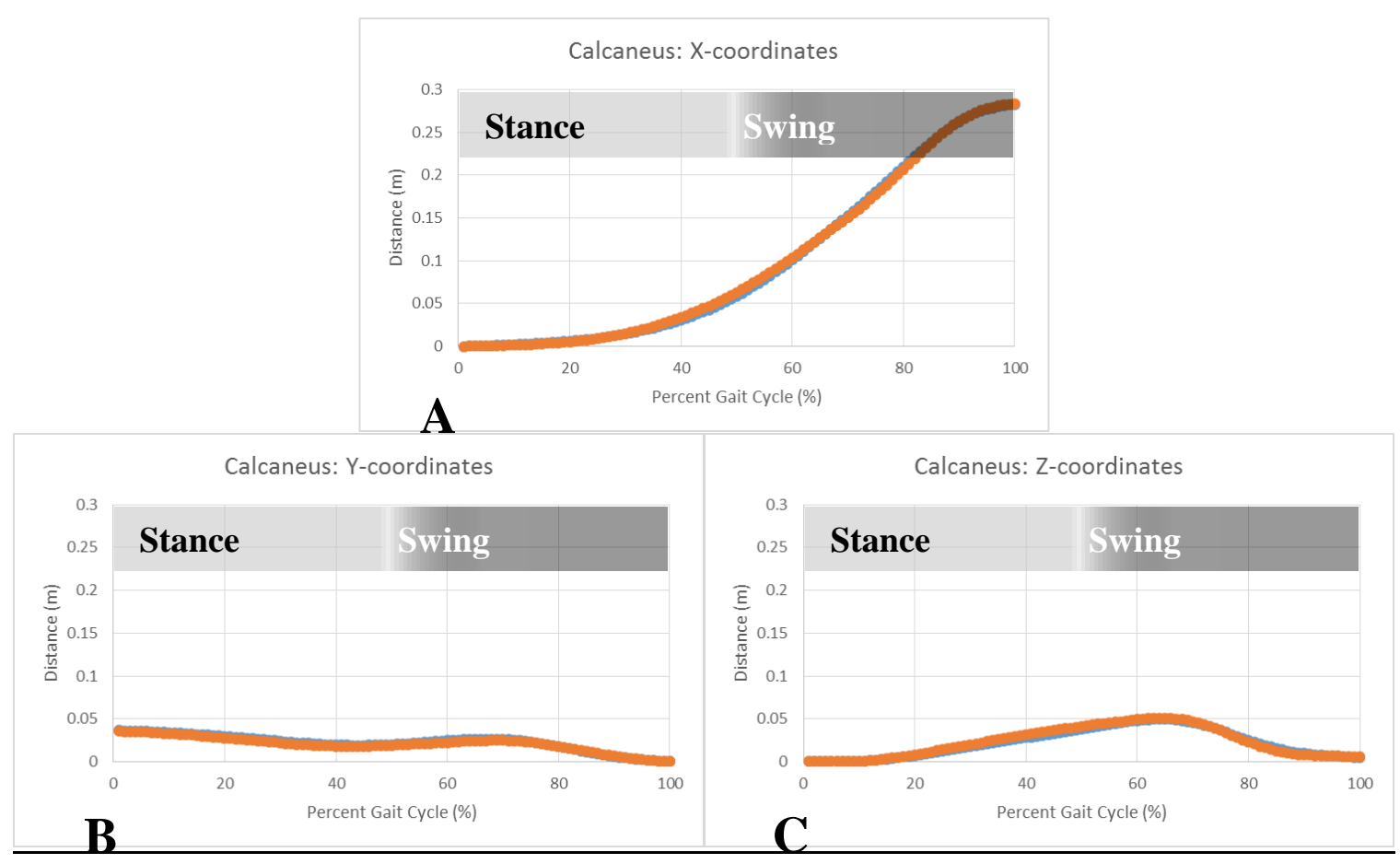

FIGURE 27- Marker displacement of the calcaneus in the A) cranial-caudal B) mediallateral and C) vertical directions. JCS marker trajectories are shown in blue, while OS marker trajectories are shown in orange. Correlation coefficients were $0.99,0.99$, and 0.99 in the $\mathrm{X}, \mathrm{Y}$, and $\mathrm{Z}$ directions respectively. The maximum total distance between JCS and $\mathrm{OS}$ marker locations was $0.53 \mathrm{~cm}$. 

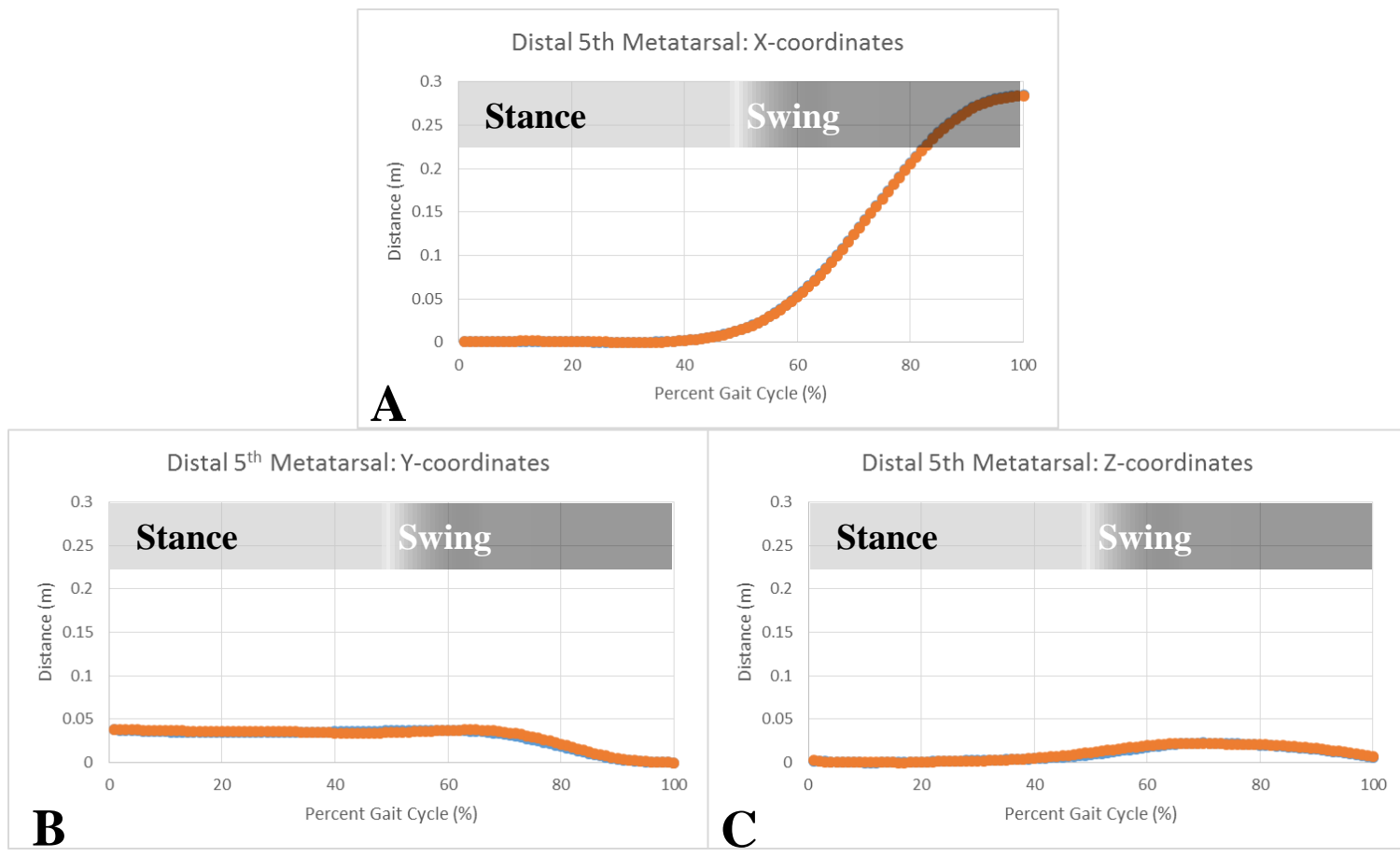

FIGURE 28 - Marker displacement of the distal $5^{\text {th }}$ metatarsal in the A) cranial-caudal B) medial-lateral and C) vertical directions. JCS marker trajectories are shown in blue, while OS marker trajectories are shown in orange. Correlation coefficients were 0.99 , 0.99 , and 0.99 in the $\mathrm{X}, \mathrm{Y}$, and $\mathrm{Z}$ directions respectively. The maximum total distance between JCS and OS marker locations was $0.36 \mathrm{~cm}$.

\section{Muscle Activation Patterns and Parametric Sensitivity Analysis}

Muscle activation patterns of a representative trial from healthy subject 1 of the baseline model are shown along with activations from the model with 10, 20, 30, and 40 percent reduction in maximum isometric force in Figures 29-45. Average activations of all healthy subjects can be seen in Appendix C. 


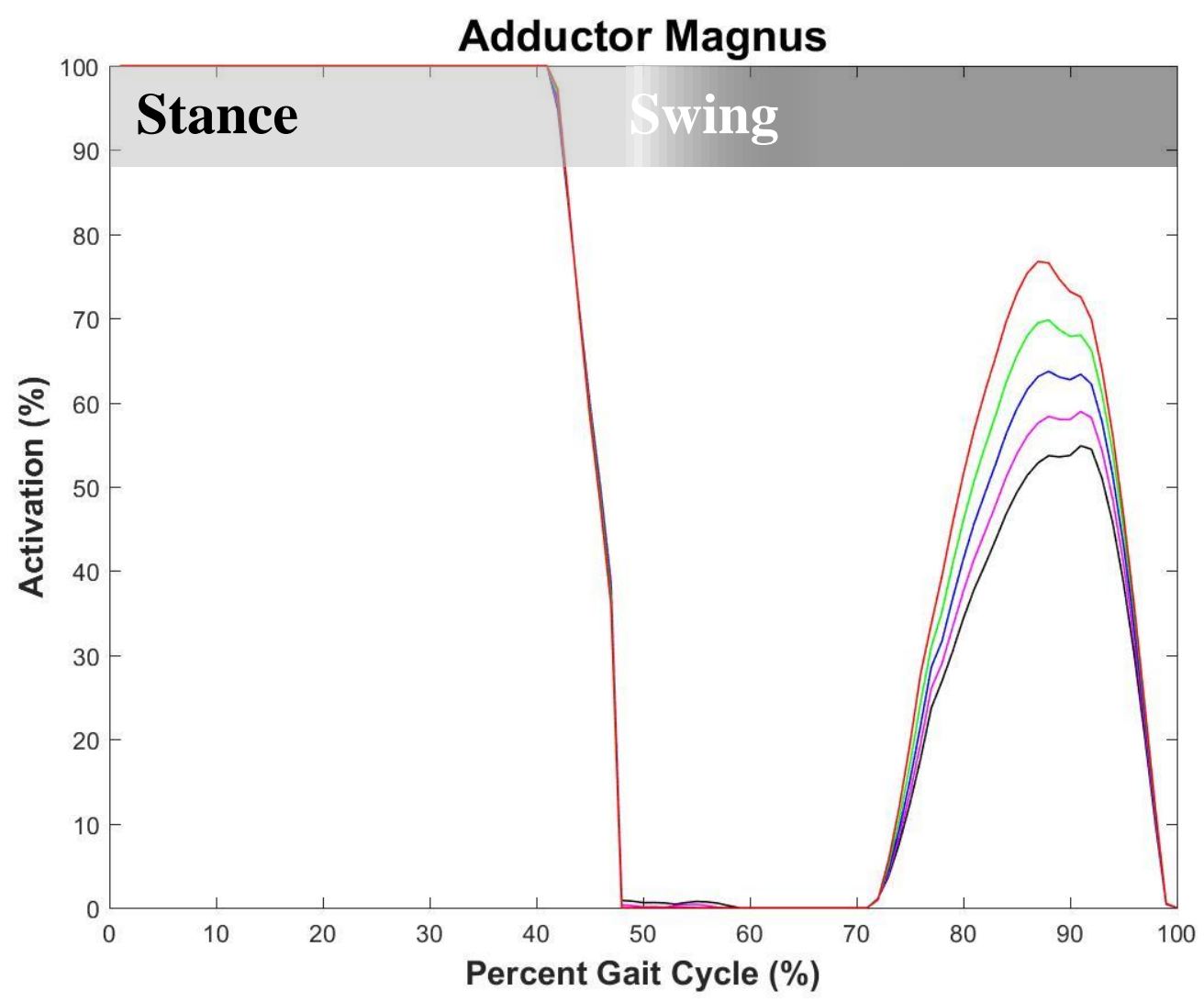

FIGURE 29 - Simulated muscle activations of the adductor magnus in healthy subject 1 .

Activation patterns predicted using the baseline model are shown in black, $10 \%$ reduction is shown in magenta, $20 \%$ reduction is shown in blue, $30 \%$ reduction is shown in green, and $40 \%$ reduction is shown in red. 


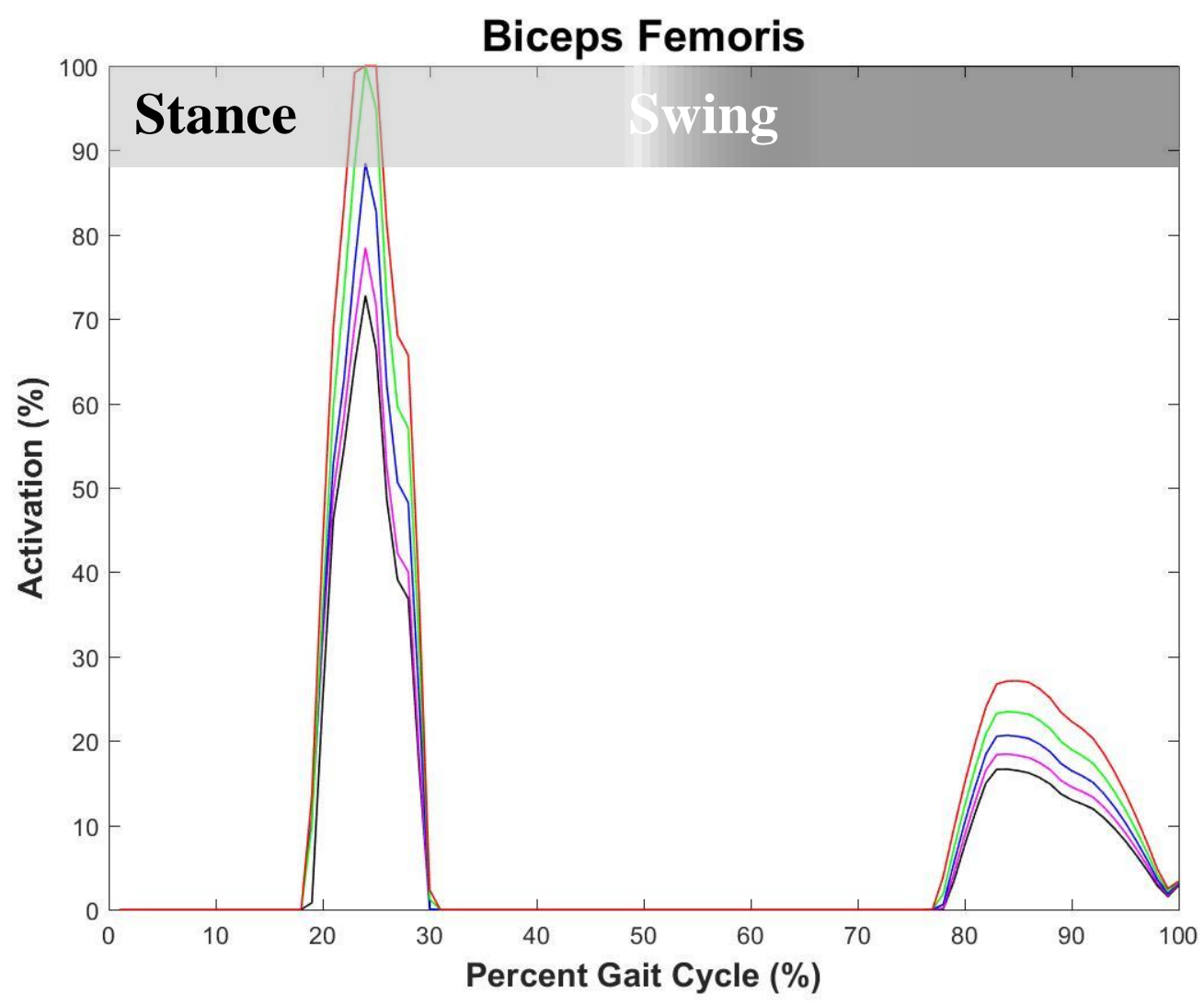

FIGURE 30 - Simulated muscle activations of the biceps femoris in healthy subject 1.

Activation patterns predicted using the baseline model are shown in black, $10 \%$ reduction is shown in magenta, $20 \%$ reduction is shown in blue, $30 \%$ reduction is shown in green, and $40 \%$ reduction is shown in red. 


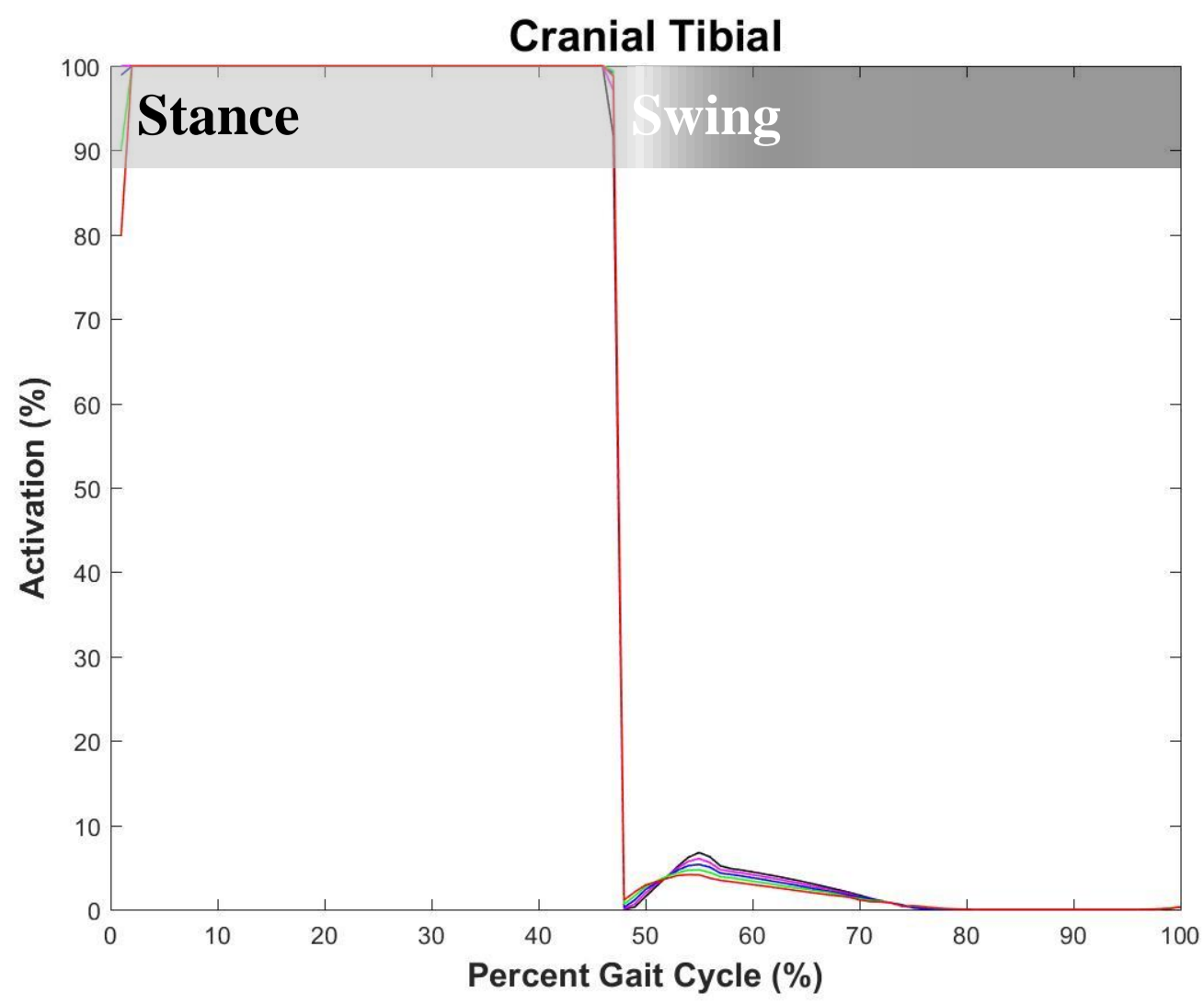

FIGURE 31 - Simulated muscle activations of the cranial tibial in healthy subject 1.

Activation patterns predicted using the baseline model are shown in black, $10 \%$ reduction is shown in magenta, $20 \%$ reduction is shown in blue, $30 \%$ reduction is shown in green, and $40 \%$ reduction is shown in red. 


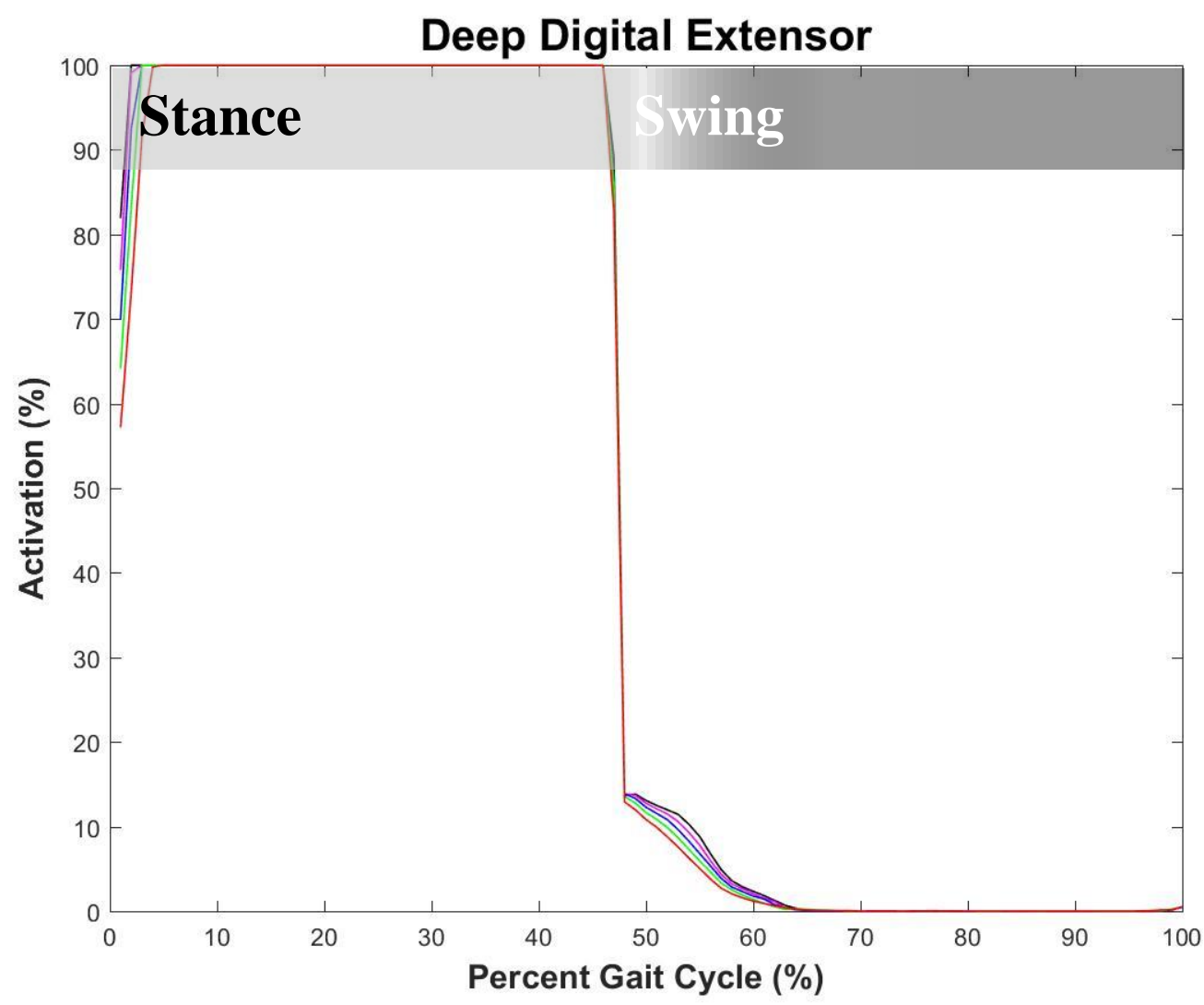

FIGURE 32 - Simulated muscle activations of the long digital extensor in healthy subject 1. Activation patterns predicted using the baseline model are shown in black, $10 \%$ reduction is shown in magenta, $20 \%$ reduction is shown in blue, $30 \%$ reduction is shown in green, and $40 \%$ reduction is shown in red. 


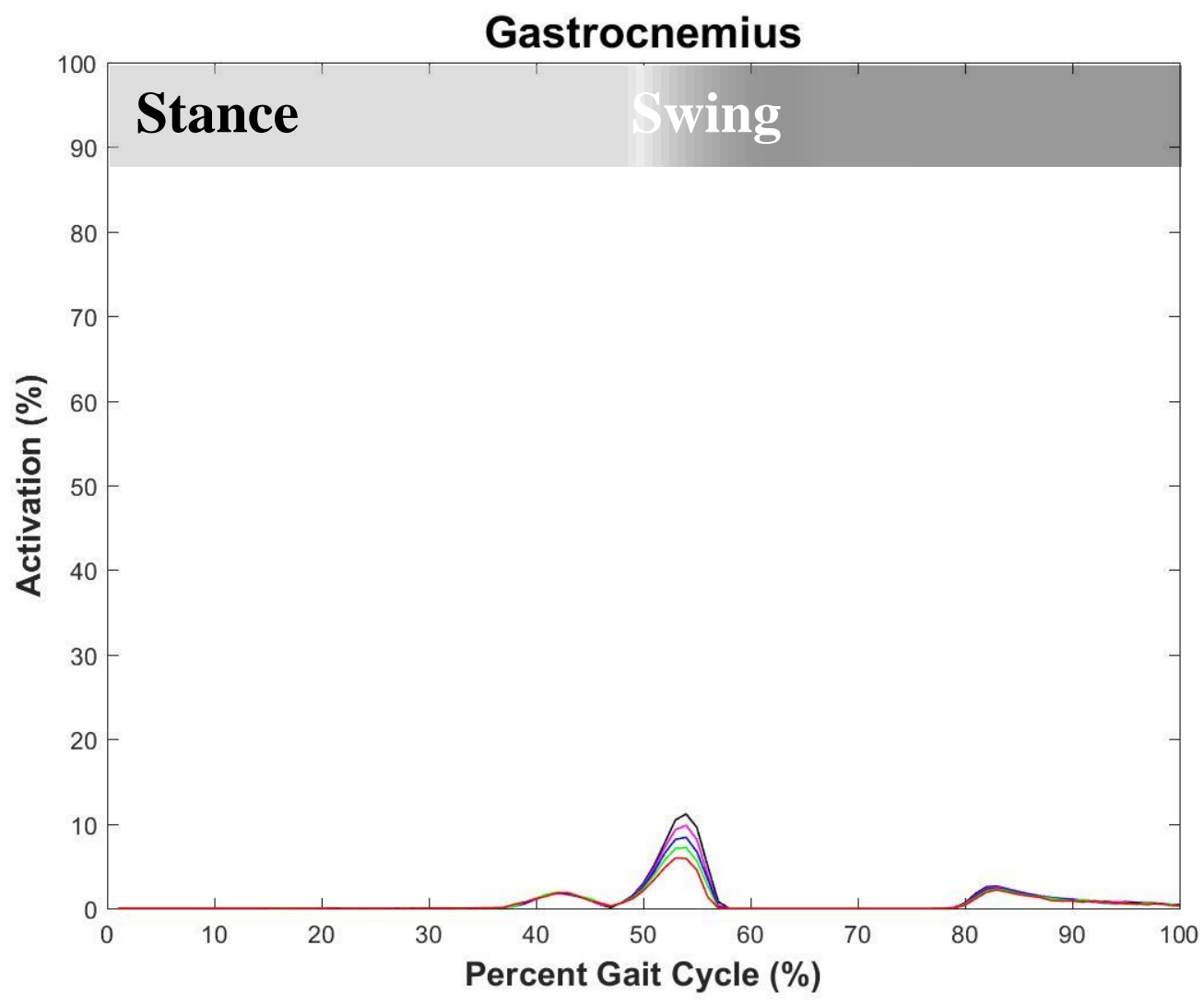

FIGURE 33 - Simulated muscle activations of the gastrocnemius in healthy subject 1.

Activation patterns predicted using the baseline model are shown in black, $10 \%$ reduction is shown in magenta, $20 \%$ reduction is shown in blue, $30 \%$ reduction is shown in green, and $40 \%$ reduction is shown in red. 


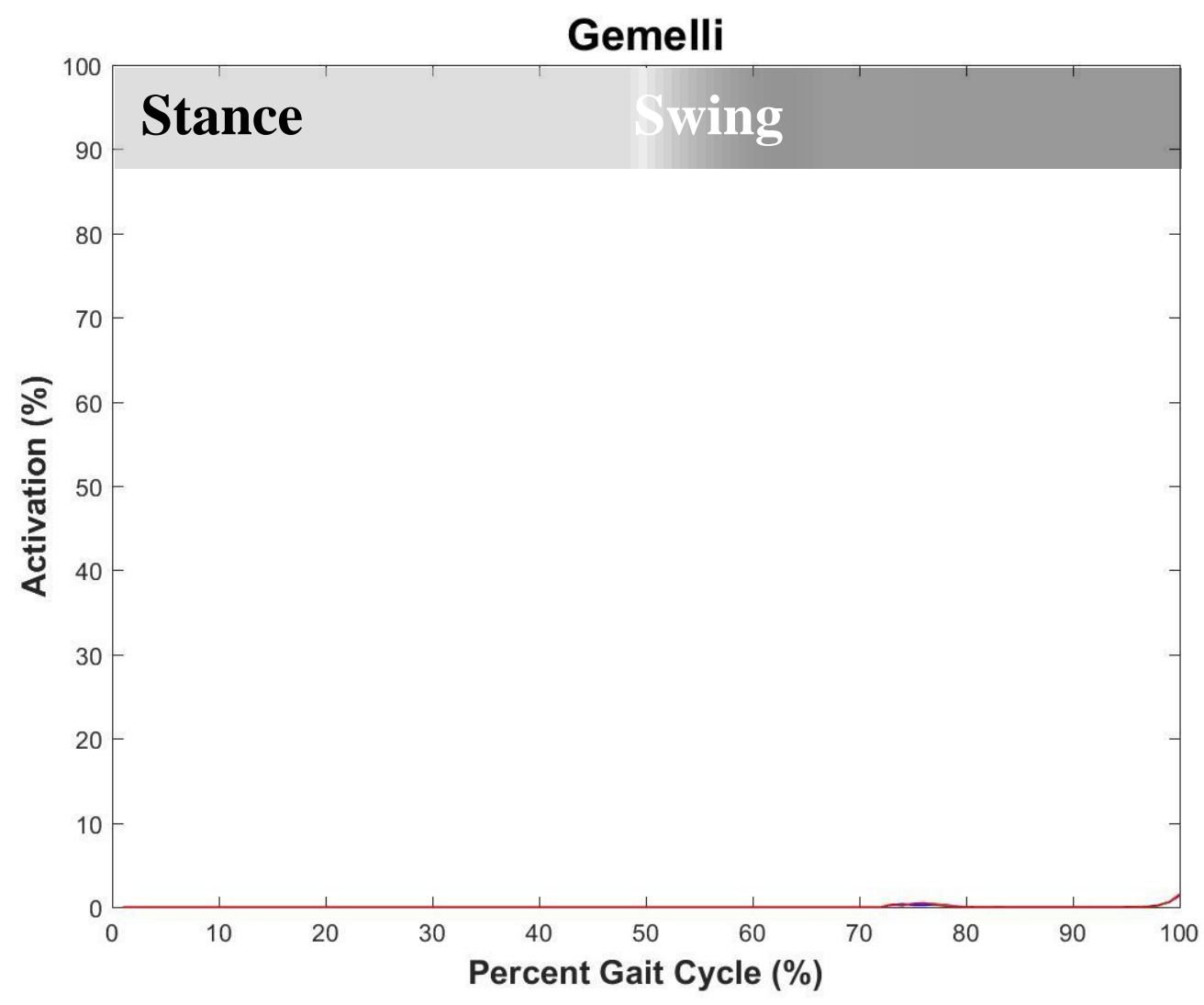

FIGURE 34 - Simulated muscle activations of the gemellus in healthy subject 1 .

Activation patterns predicted using the baseline model are shown in black, $10 \%$ reduction is shown in magenta, $20 \%$ reduction is shown in blue, $30 \%$ reduction is shown in green, and $40 \%$ reduction is shown in red. 


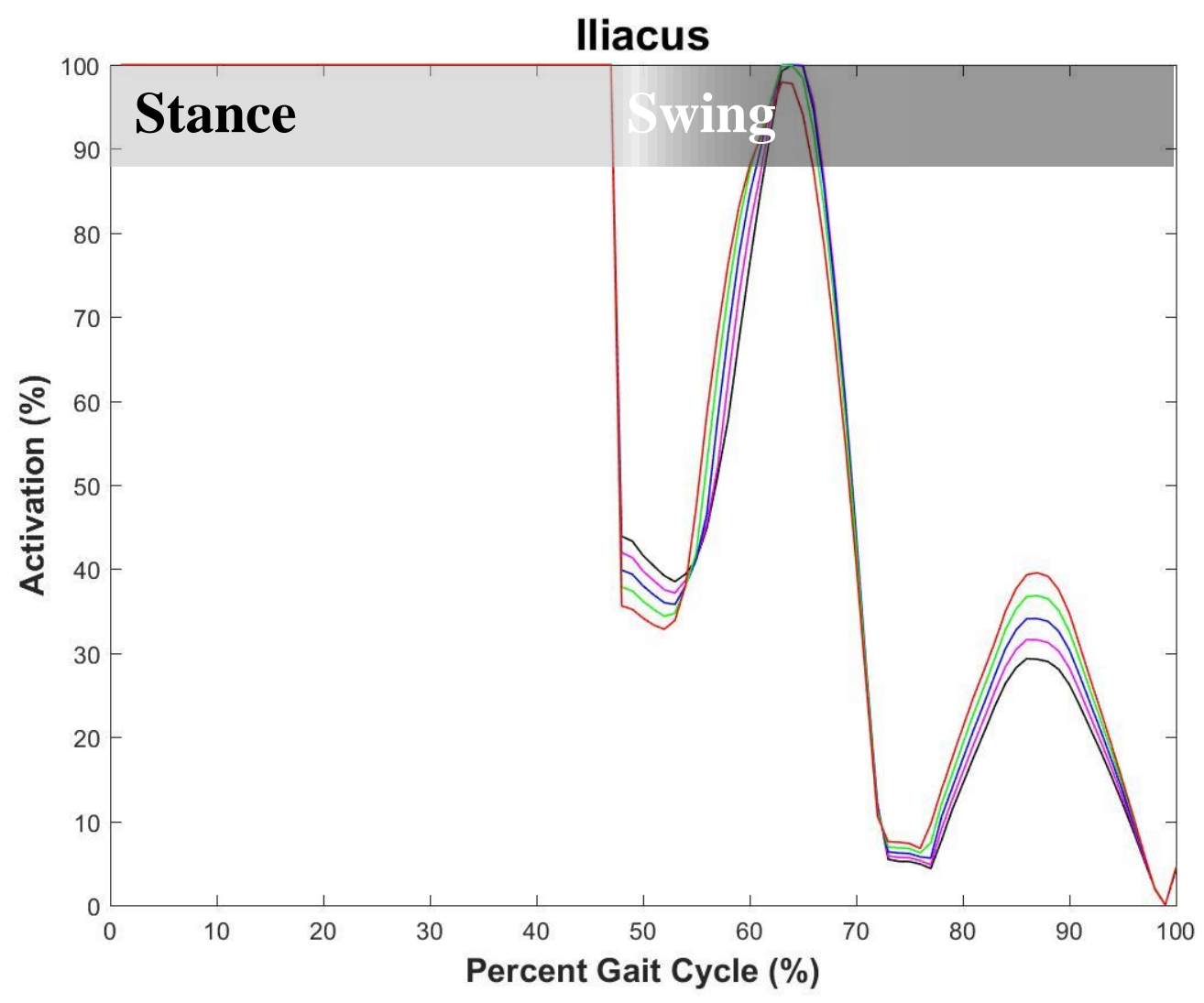

FIGURE 35 - Simulated muscle activations of the iliacus in healthy subject 1 . Activation patterns predicted using the baseline model are shown in black, $10 \%$ reduction is shown in magenta, $20 \%$ reduction is shown in blue, $30 \%$ reduction is shown in green, and $40 \%$ reduction is shown in red. 


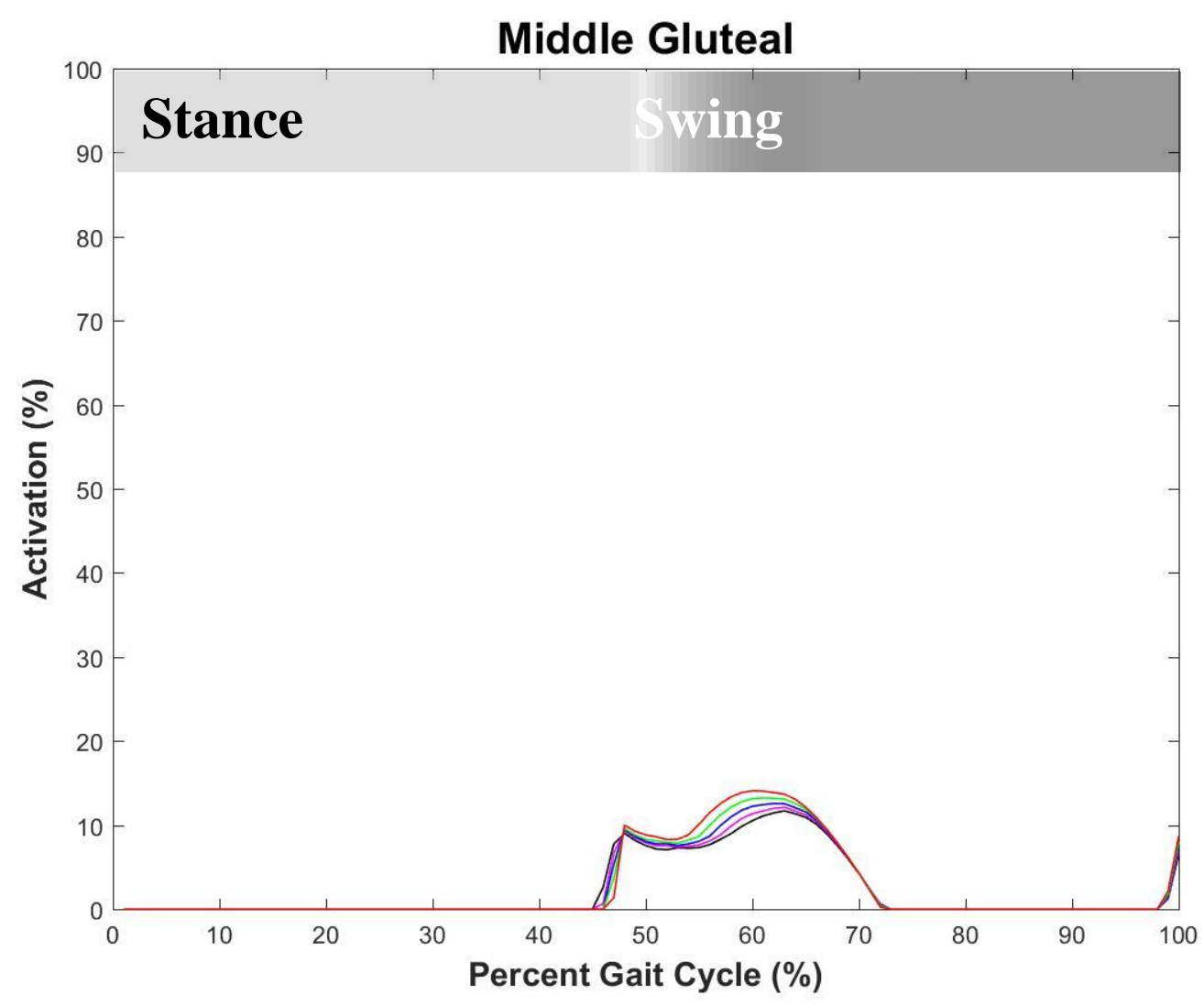

FIGURE 36 - Simulated muscle activations of the middle gluteus in thealthy subject 1.

Activation patterns predicted using the baseline model are shown in black, $10 \%$ reduction is shown in magenta, $20 \%$ reduction is shown in blue, $30 \%$ reduction is shown in green, and $40 \%$ reduction is shown in red. 


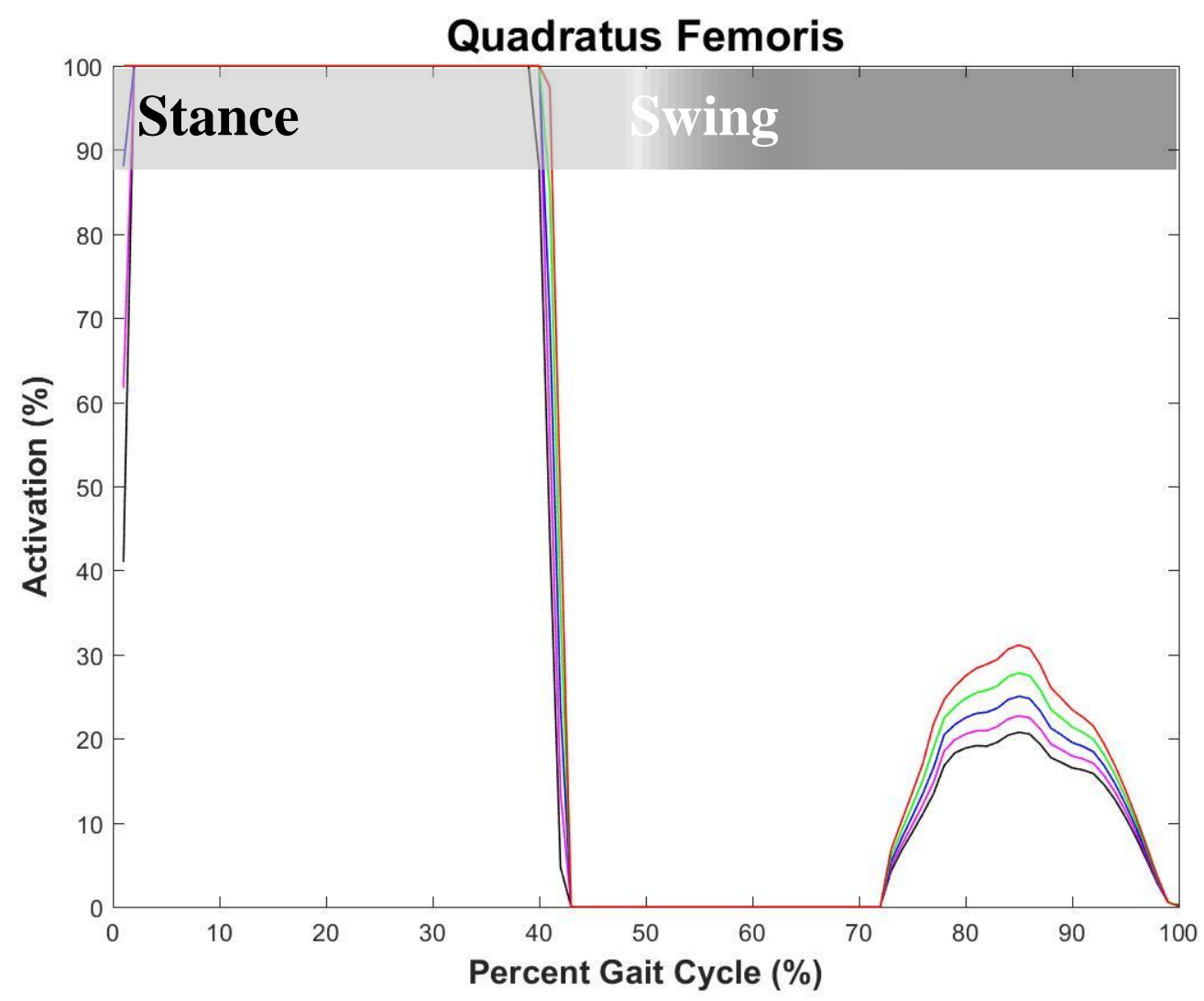

FIGURE 37 - Simulated muscle activations of the quadratus femoris in healthy subject 1. Activation patterns predicted using the baseline model are shown in black, $10 \%$ reduction is shown in magenta, $20 \%$ reduction is shown in blue, $30 \%$ reduction is shown in green, and $40 \%$ reduction is shown in red. 


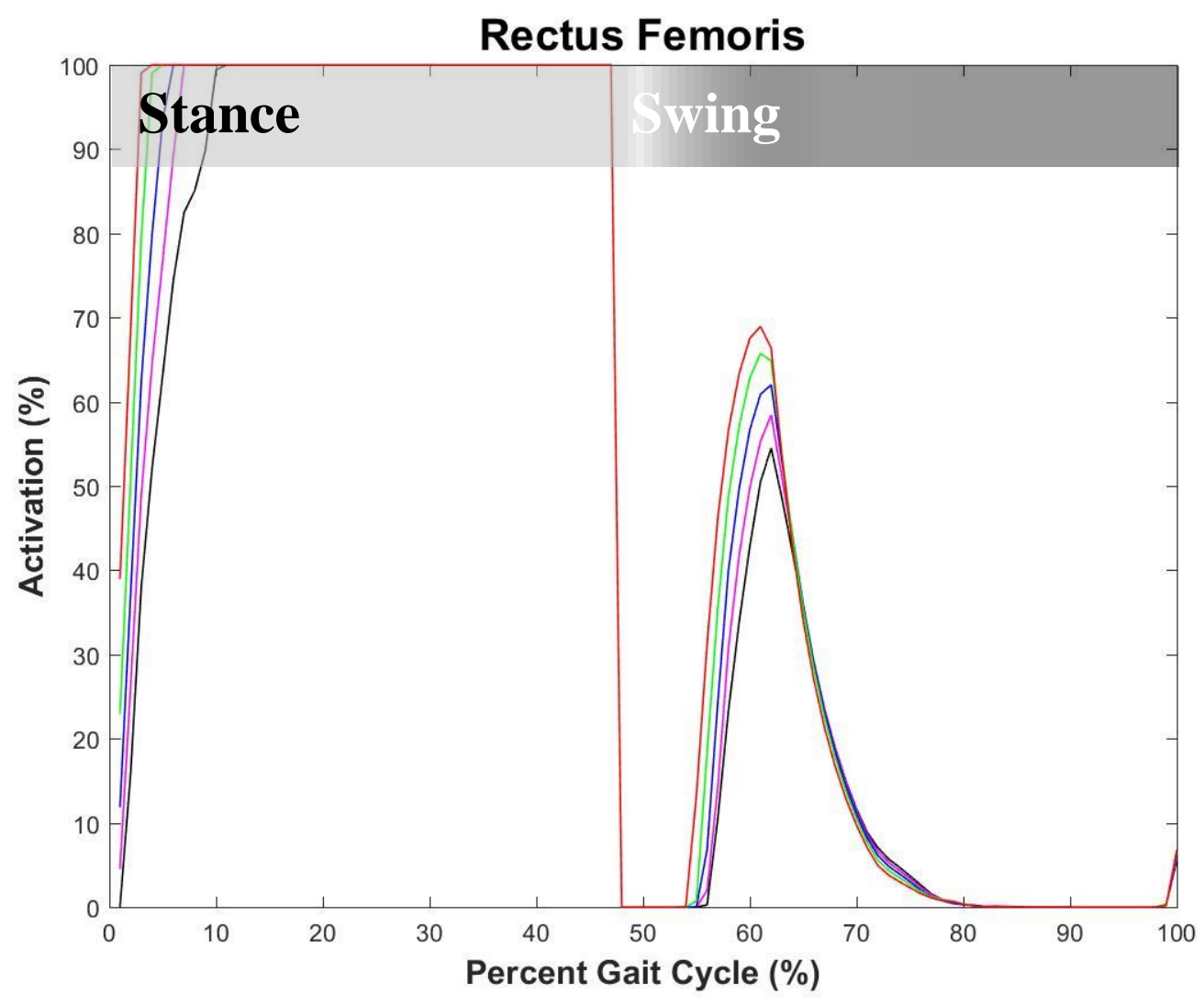

FIGURE 38 - Simulated muscle activations of the rectus femoris in healthy subject 1.

Activation patterns predicted using the baseline model are shown in black, $10 \%$ reduction is shown in magenta, $20 \%$ reduction is shown in blue, $30 \%$ reduction is shown in green, and $40 \%$ reduction is shown in red. 


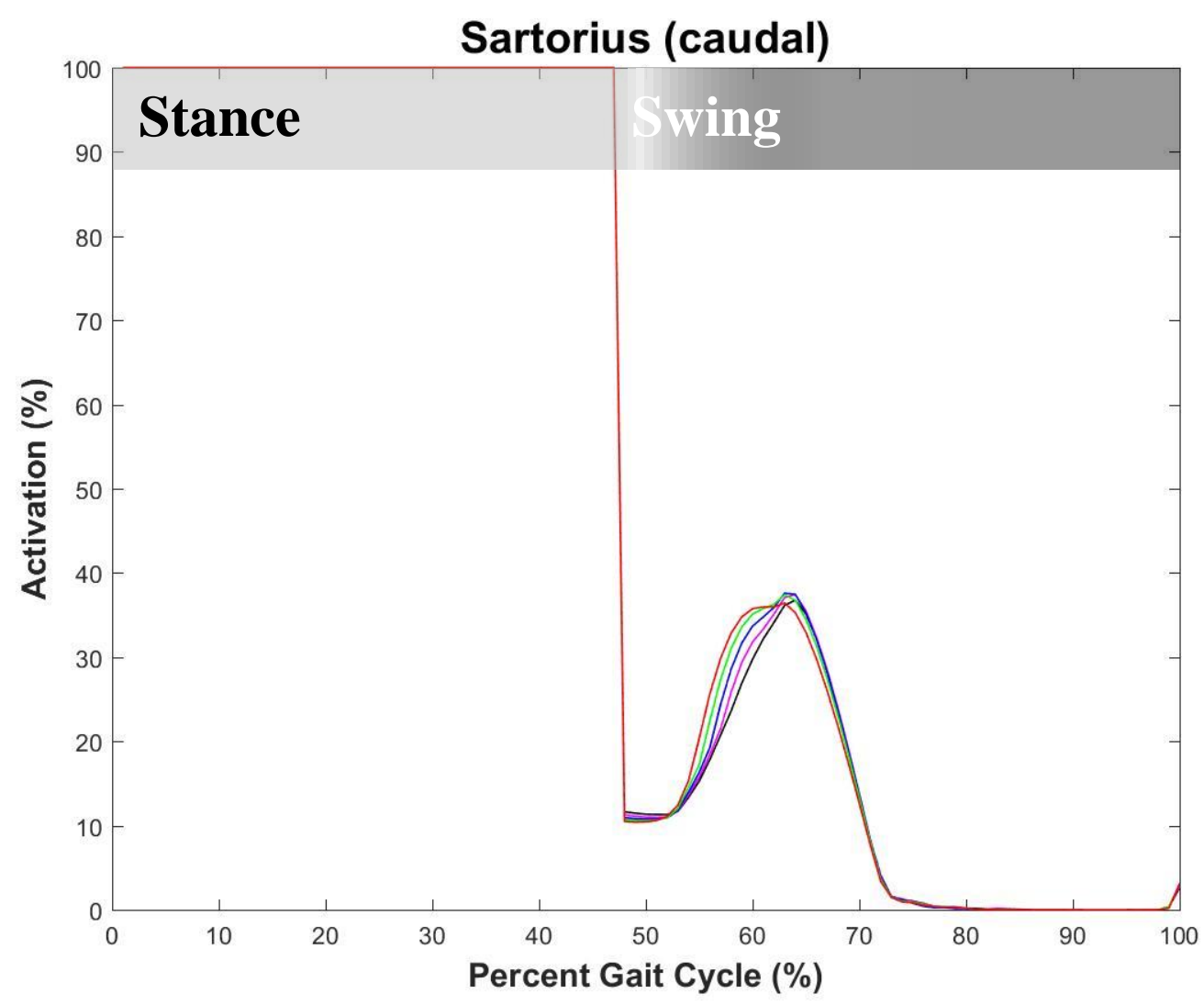

FIGURE 39 - Simulated muscle activations of the caudal Sartorius in healthy subject 1.

Activation patterns predicted using the baseline model are shown in black, $10 \%$ reduction is shown in magenta, $20 \%$ reduction is shown in blue, $30 \%$ reduction is shown in green, and $40 \%$ reduction is shown in red. 


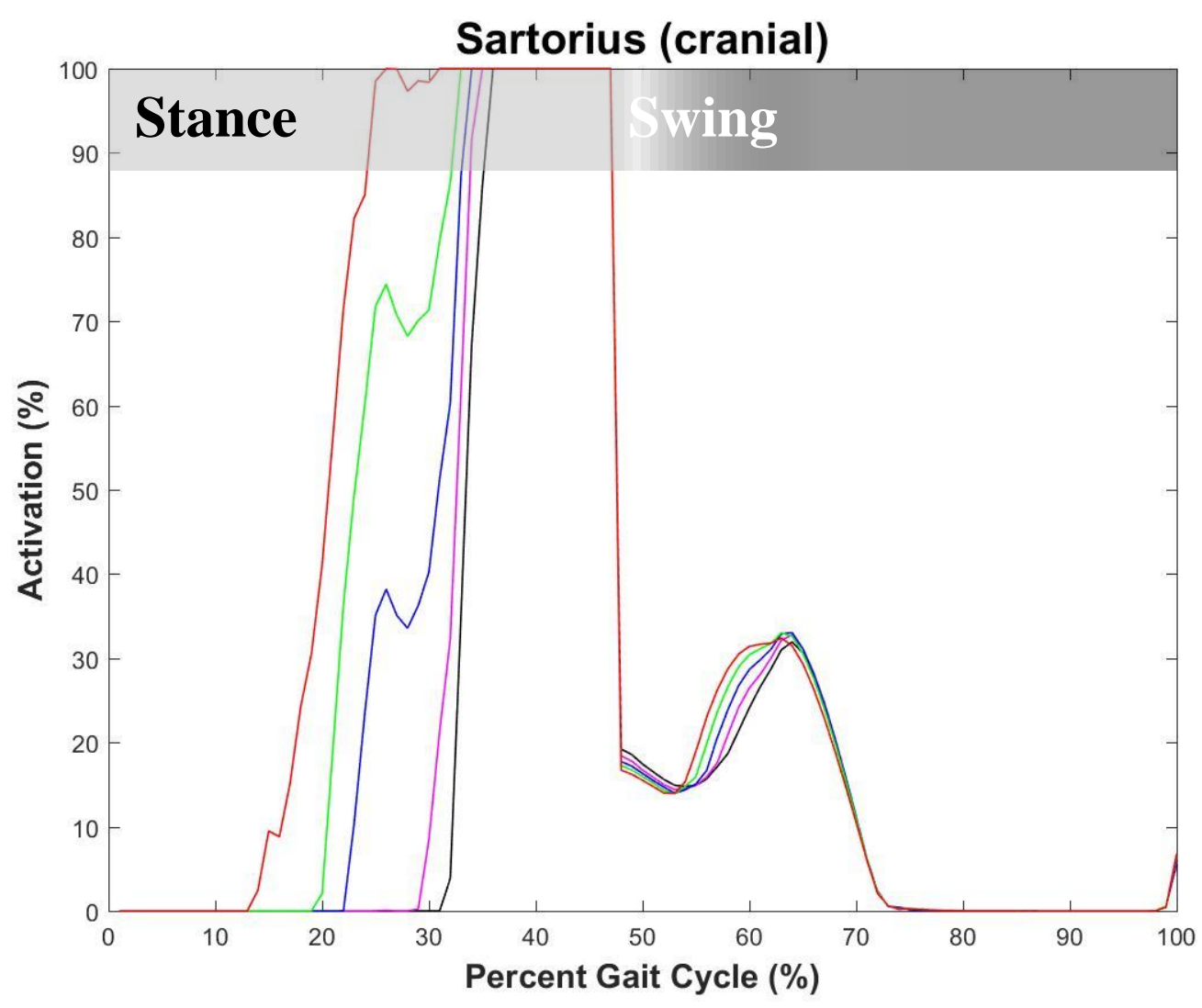

FIGURE 40 - Simulated muscle activations of the cranial Sartorius in healthy subject 1.

Activation patterns predicted using the baseline model are shown in black, $10 \%$ reduction is shown in magenta, $20 \%$ reduction is shown in blue, $30 \%$ reduction is shown in green, and $40 \%$ reduction is shown in red. 


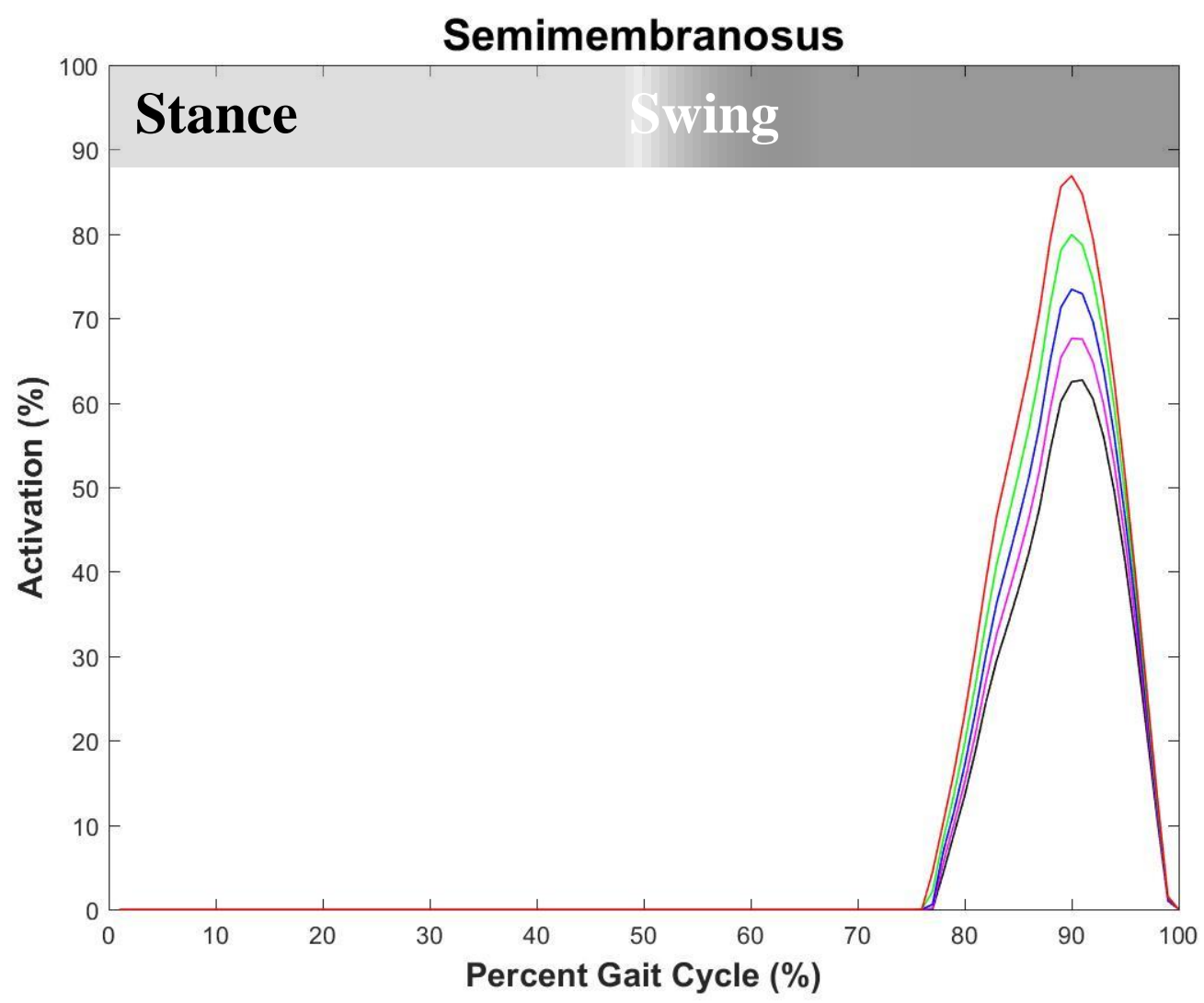

FIGURE 41 - Simulated muscle activations of the semimembranosus in healthy subject 1.

Activation patterns predicted using the baseline model are shown in black, $10 \%$ reduction is shown in magenta, $20 \%$ reduction is shown in blue, $30 \%$ reduction is shown in green, and $40 \%$ reduction is shown in red. 


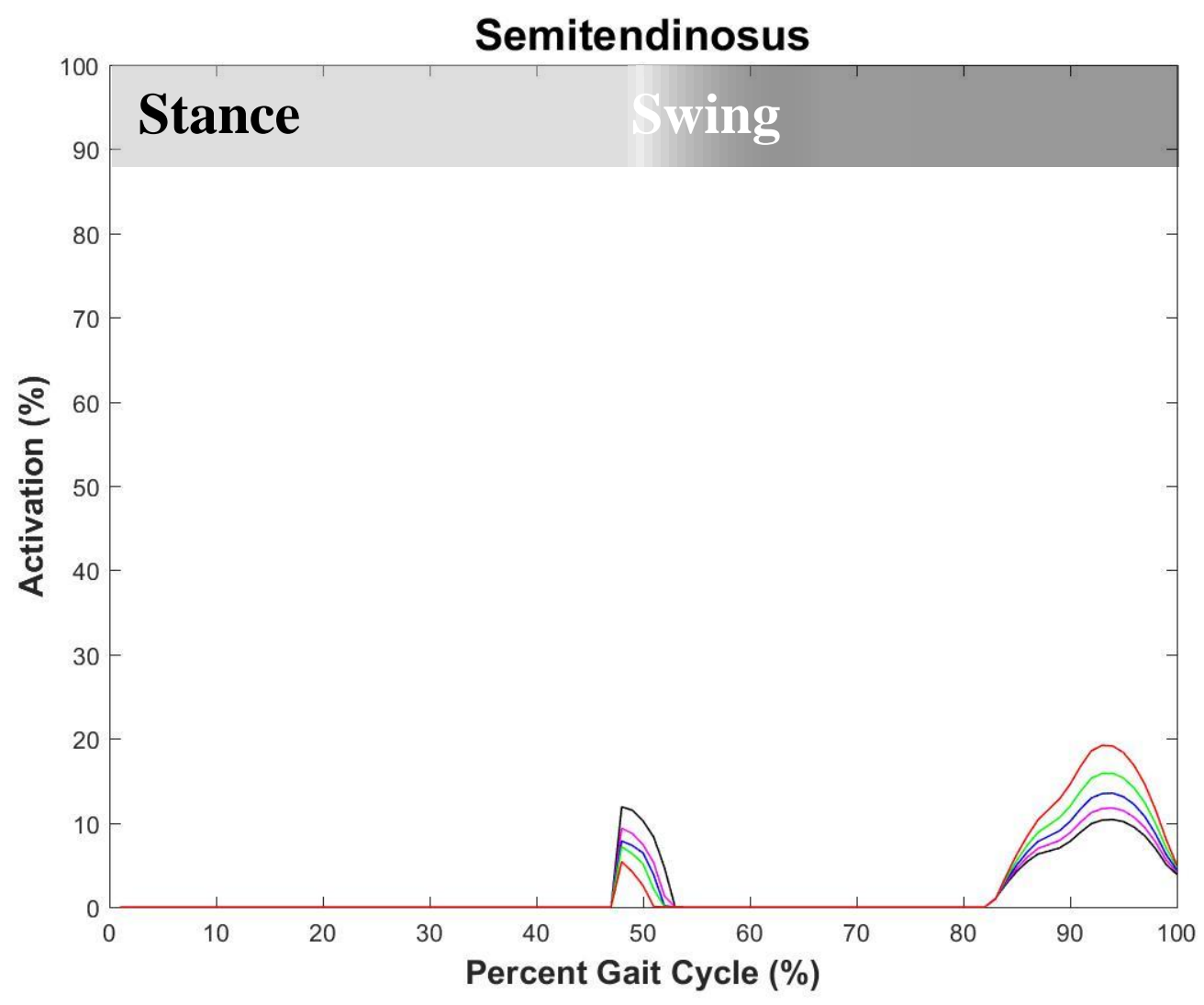

FIGURE 42 - Simulated muscle activations of the semitendinosus in healthy subject 1.

Activation patterns predicted using the baseline model are shown in black, $10 \%$ reduction is shown in magenta, $20 \%$ reduction is shown in blue, $30 \%$ reduction is shown in green, and $40 \%$ reduction is shown in red. 


\section{Tensor Fascia Lata}

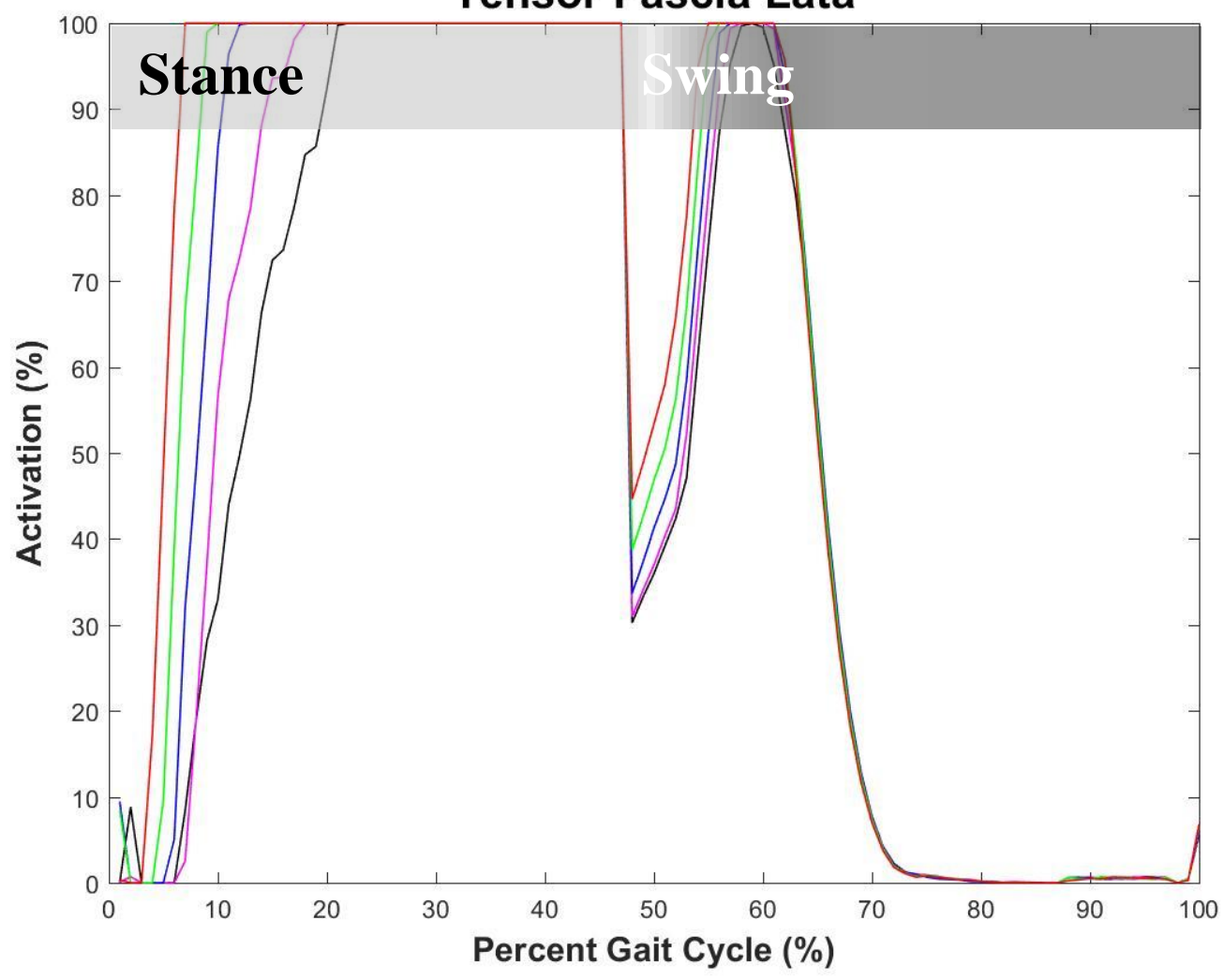

FIGURE 43 - Simulated muscle activations of the tensor fascia lata in healthy subject 1.

Activation patterns predicted using the baseline model are shown in black, $10 \%$ reduction is shown in magenta, $20 \%$ reduction is shown in blue, $30 \%$ reduction is shown in green, and $40 \%$ reduction is shown in red. 


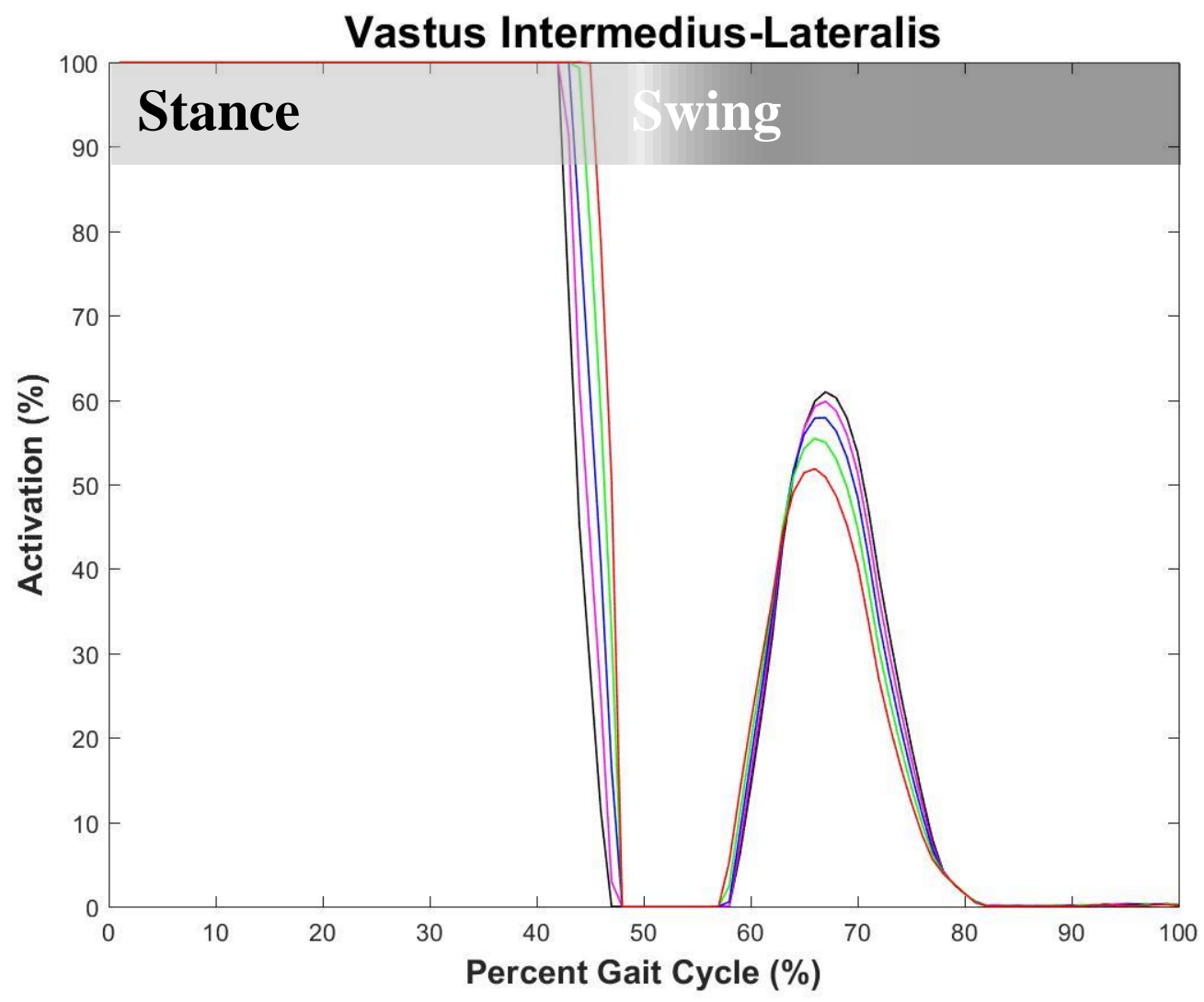

FIGURE 44 - Simulated muscle activations of the vastus intermedius/lateralis in healthy subject 1 . Activation patterns predicted using the baseline model are shown in black, $10 \%$ reduction is shown in magenta, $20 \%$ reduction is shown in blue, $30 \%$ reduction is shown in green, and $40 \%$ reduction is shown in red. 


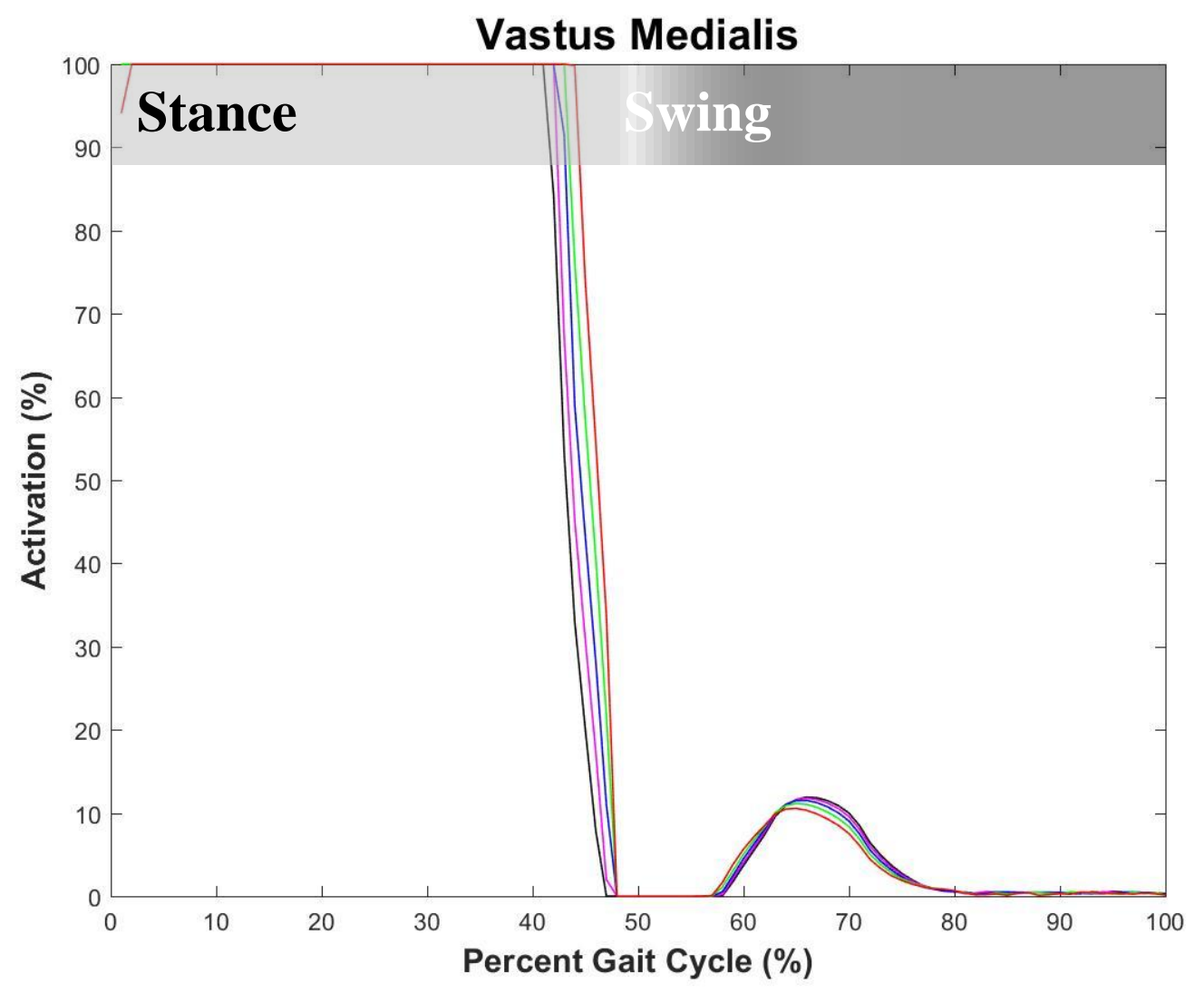

FIGURE 45 - Simulated muscle activations of the vastus medialis in healthy subject 1. Activation patterns predicted using the baseline model are shown in black, $10 \%$ reduction is shown in magenta, $20 \%$ reduction is shown in blue, $30 \%$ reduction is shown in green, and $40 \%$ reduction is shown in red.

\section{E. Parametric Sensitivity Analysis}

Muscle activations were compared to baseline for changes in maximum isometric force. Values of the sensitivity index are shown in Tables IX and X. Higher absolute values of sensitivity indices suggest a greater influence on the outcome. Positive sensitivity index values indicate increasing peak muscle activation, while negative values 
indicate decreasing peak muscle activation. Data from these tables are presented in graphical form in Figures 46 and 47.

TABLE IX

SENSITIVITY INDICES DESCRIBING INFLUENCE OF MAXIMUM ISOMETRIC

FORCE ON PEAK MUSCLE ACTIVATION FOR EACH MUSCLE DURING

STANCE.

\begin{tabular}{|c|c|c|c|c|}
\hline Muscle & $\begin{array}{c}10 \% \\
\text { Reduction }\end{array}$ & $\begin{array}{c}20 \% \\
\text { Reduction } \\
\end{array}$ & $\begin{array}{c}\mathbf{3 0 \%} \\
\text { Reduction }\end{array}$ & $\begin{array}{c}40 \% \\
\text { Reduction }\end{array}$ \\
\hline Adductor Magnus & 0 & 0 & 0 & 0 \\
\hline Biceps Femoris & 0.8 & 1.1 & 1.2 & 0.9 \\
\hline Cranial Tibial & 0 & 0 & 0 & 0 \\
\hline Long Digital Extensor & 0 & 0 & 0 & 0 \\
\hline Gastrocnemius & -0.6 & -0.5 & -0.3 & -0.1 \\
\hline Gemellus & 0.4 & 0.6 & 1 & 1.4 \\
\hline Iliacus & 0 & 0 & 0 & 0 \\
\hline Middle Gluteal & 0.3 & 0.3 & 0.3 & 0.3 \\
\hline Quadratus Femoris & 0 & 0 & 0 & 0 \\
\hline Rectus Femoris & 0 & 0 & 0 & 0 \\
\hline Sarotius (caudal) & 0 & 0 & 0 & 0 \\
\hline Sartorius (cranial) & 0 & 0 & 0 & 0 \\
\hline Semimembranosus & -4 & -1.9 & -1.2 & -0.9 \\
\hline Semitendinosus & -2.1 & -1.3 & -1.2 & -1.3 \\
\hline Tensor Facia Lata & 0 & 0 & 0 & 0 \\
\hline Vastus Intermedius/Lateralis & 0 & 0 & 0 & 0 \\
\hline Vastus Medialis & 0 & 0 & 0 & 0 \\
\hline
\end{tabular}




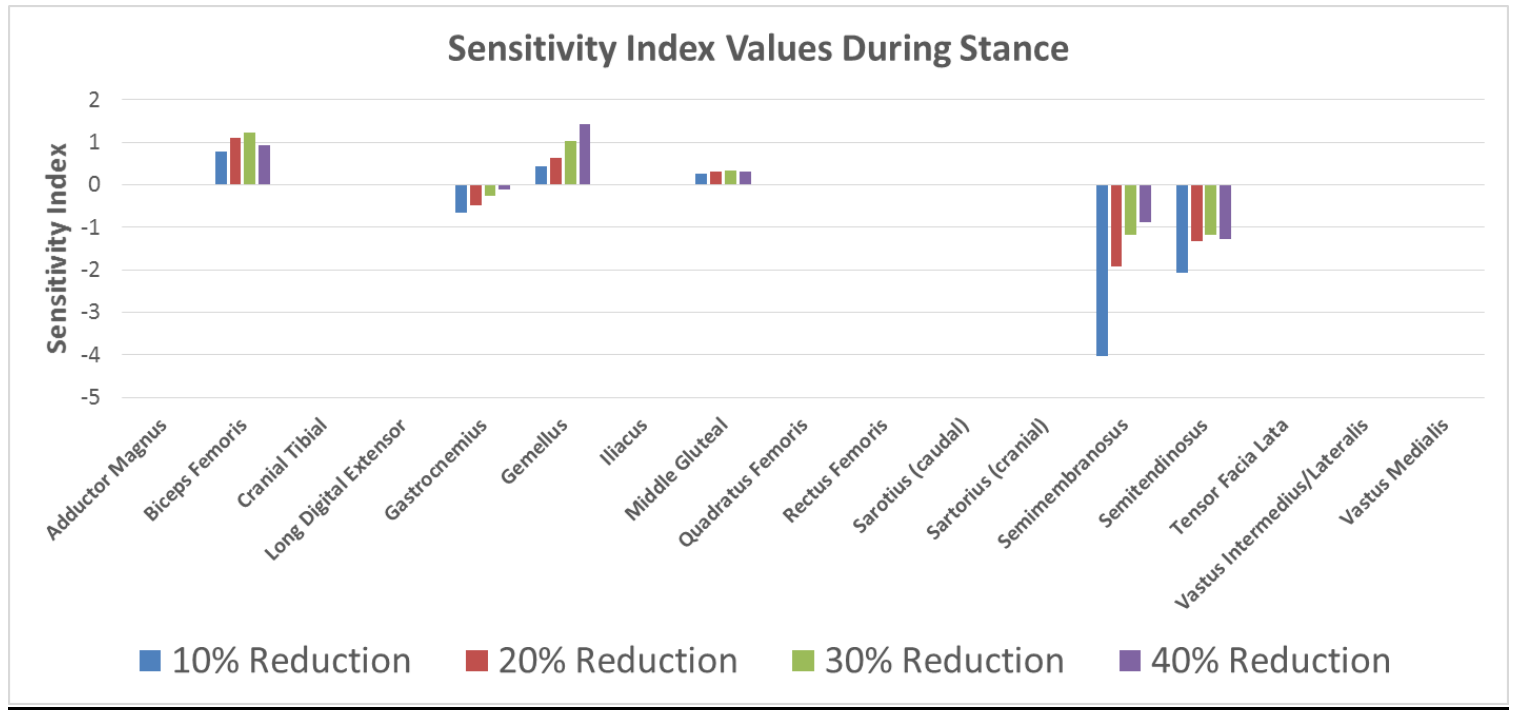

FIGURE 46 - Sensitivity indices describing influence of maximum isometric force on peak muscle activation for each muscle during stance.

TABLE X

SENSITIVITY INDICES DESCRIBING INFLUENCE OF MAXIMUM ISOMETRIC

FORCE ON PEAK MUSCLE ACTIVATION FOR EACH MUSCLE DURING SWING.

\begin{tabular}{|c|c|c|c|c|}
\hline Muscle & $\begin{array}{c}10 \% \\
\text { Reduction }\end{array}$ & $\begin{array}{c}20 \% \\
\text { Reduction }\end{array}$ & $\begin{array}{c}30 \% \\
\text { Reduction }\end{array}$ & $\begin{array}{c}40 \% \\
\text { Reduction }\end{array}$ \\
\hline Adductor Magnus & 0.7 & 0.7 & 0.9 & 1 \\
\hline Biceps Femoris & 1.1 & 1.2 & 1.4 & 1.6 \\
\hline Cranial Tibial & -1.1 & -1 & -1 & -1 \\
\hline Long Digital Extensor & -0.2 & -0.3 & -0.3 & -0.4 \\
\hline Gastrocnemius & -1.2 & -1.2 & -1.2 & -1.1 \\
\hline Gemellus & 0.2 & 0.4 & 0.4 & 0.4 \\
\hline Iliacus & 0 & 0 & 0 & 0 \\
\hline Middle Gluteal & 0.4 & 0.4 & 0.4 & 0.5 \\
\hline Quadratus Femoris & 0.9 & 1 & 1.1 & 1.2 \\
\hline Rectus Femoris & 0.7 & 0.7 & 0.7 & 0.7 \\
\hline Sarotius (caudal) & 0.2 & 0.1 & 0.1 & 0 \\
\hline Sartorius (cranial) & 0.3 & 0.2 & 0.1 & 0 \\
\hline Semimembranosus & 0.8 & 0.9 & 0.9 & 1 \\
\hline Semitendinosus & 0.9 & 1.3 & 1.6 & 2 \\
\hline Tensor Facia Lata & 0 & 0 & 0 & 0 \\
\hline
\end{tabular}


April 12, 2016

\begin{tabular}{|l|c|c|c|c|}
\hline Vastus Intermedius/Lateralis & -0.2 & -0.2 & -0.3 & -0.4 \\
\hline Vastus Medialis & -0.1 & -0.2 & -0.2 & -0.3 \\
\hline
\end{tabular}

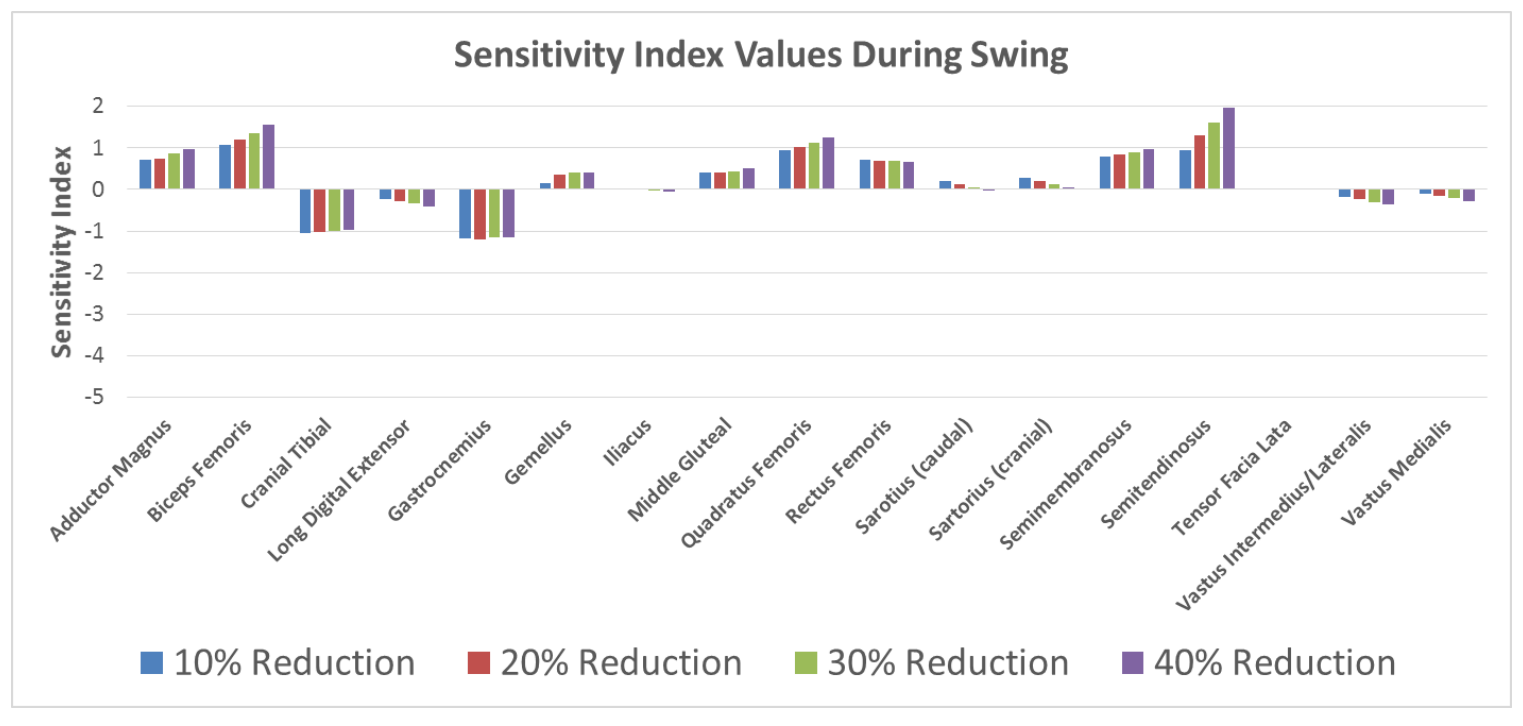

FIGURE 47 - Sensitivity index values for muscles during swing. 


\section{$\underline{\text { F. Residual and Ground Reaction Forces }}$}

Vertical ground reaction force for the representative trial of healthy subject 1 is shown in Figure 48 . The peak ground reaction force $28.9 \mathrm{~N}$, or $55 \%$ body weight.

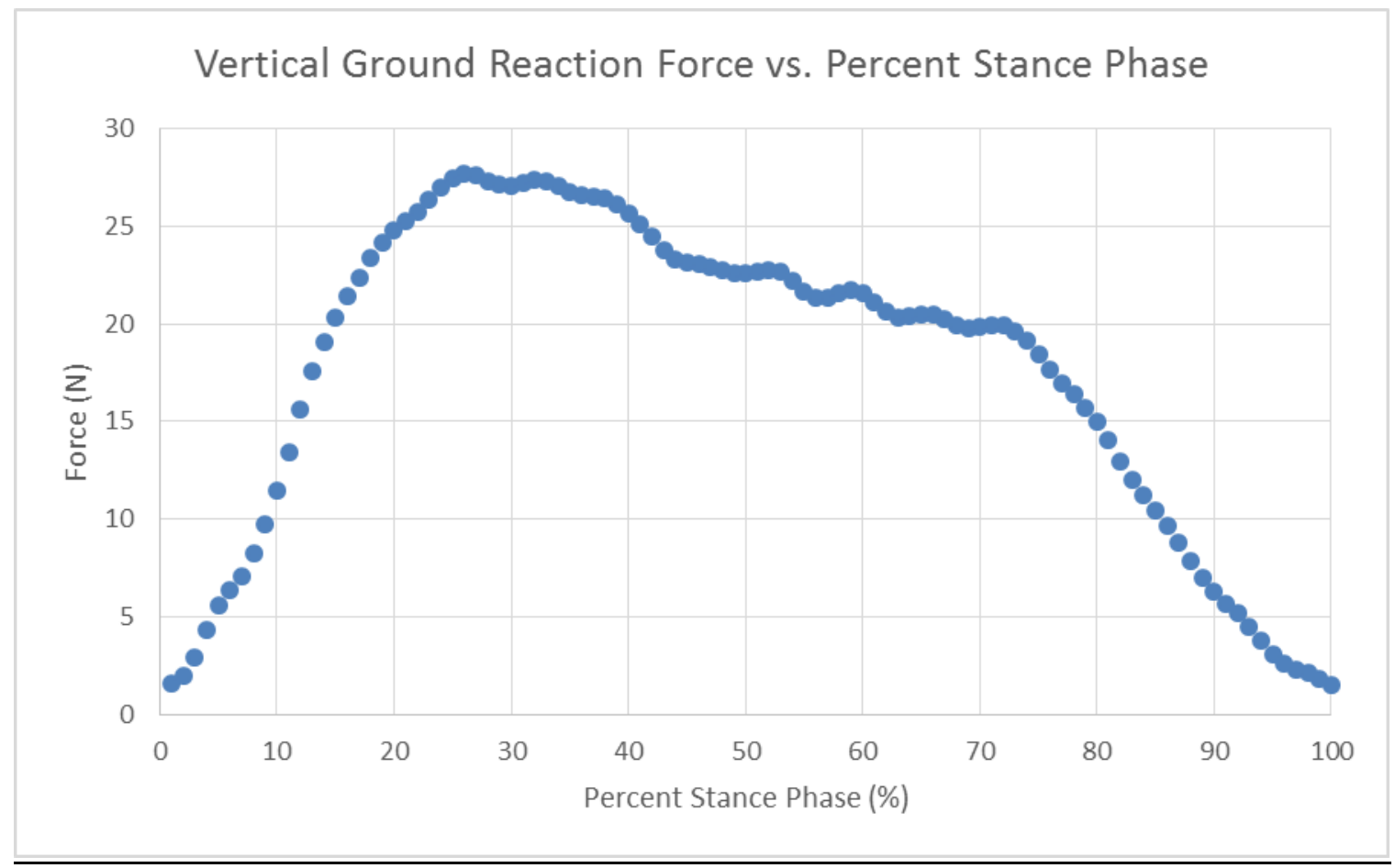

FIGURE 48 -Vertical ground reaction force for the left limb, where positive and negative values correspond to the dorsal and ventral directions, respectively.

Residual force applied to the pelvis during the residual reduction algorithm is shown in Figure 49. Forces are applied to the center of mass, however direction is defined relative to a global coordinate system. 


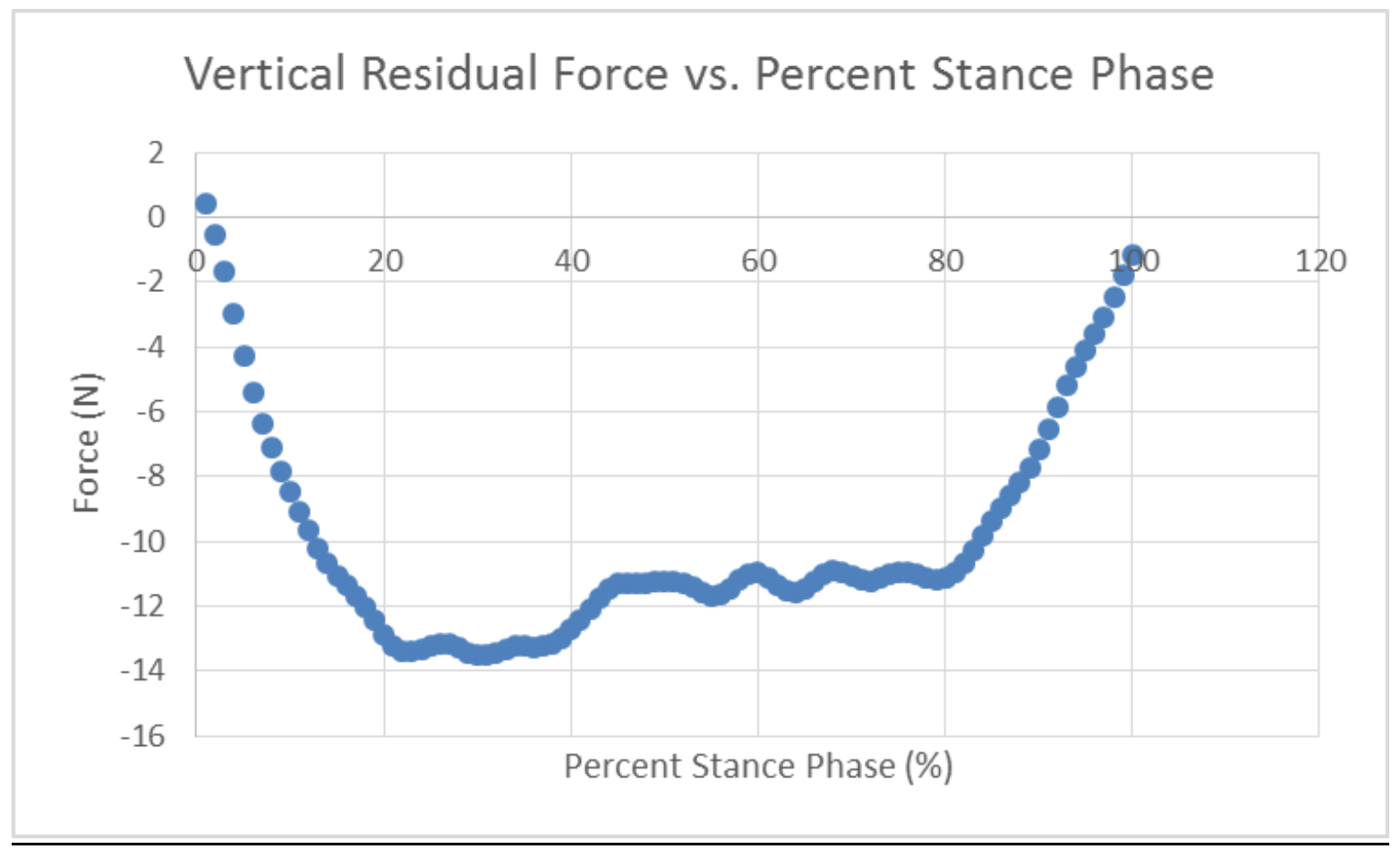

FIGURE 49 - Vertical residual force (applied to pelvis center of mass) during stance and swing, where positive and negative values correspond to the dorsal and ventral directions, respectively.

Joint moments for the hip, stifle, and tarsus are shown in Figures 50-54. Hip moment is divided into abduction/adduction, internal/external rotation, and flexion/extension based on the three degrees of freedom in the modeled hip joint. Stifle and tarsus moments are limited to flexion/extension based on modeled joint constraints (i.e. pin joint). 


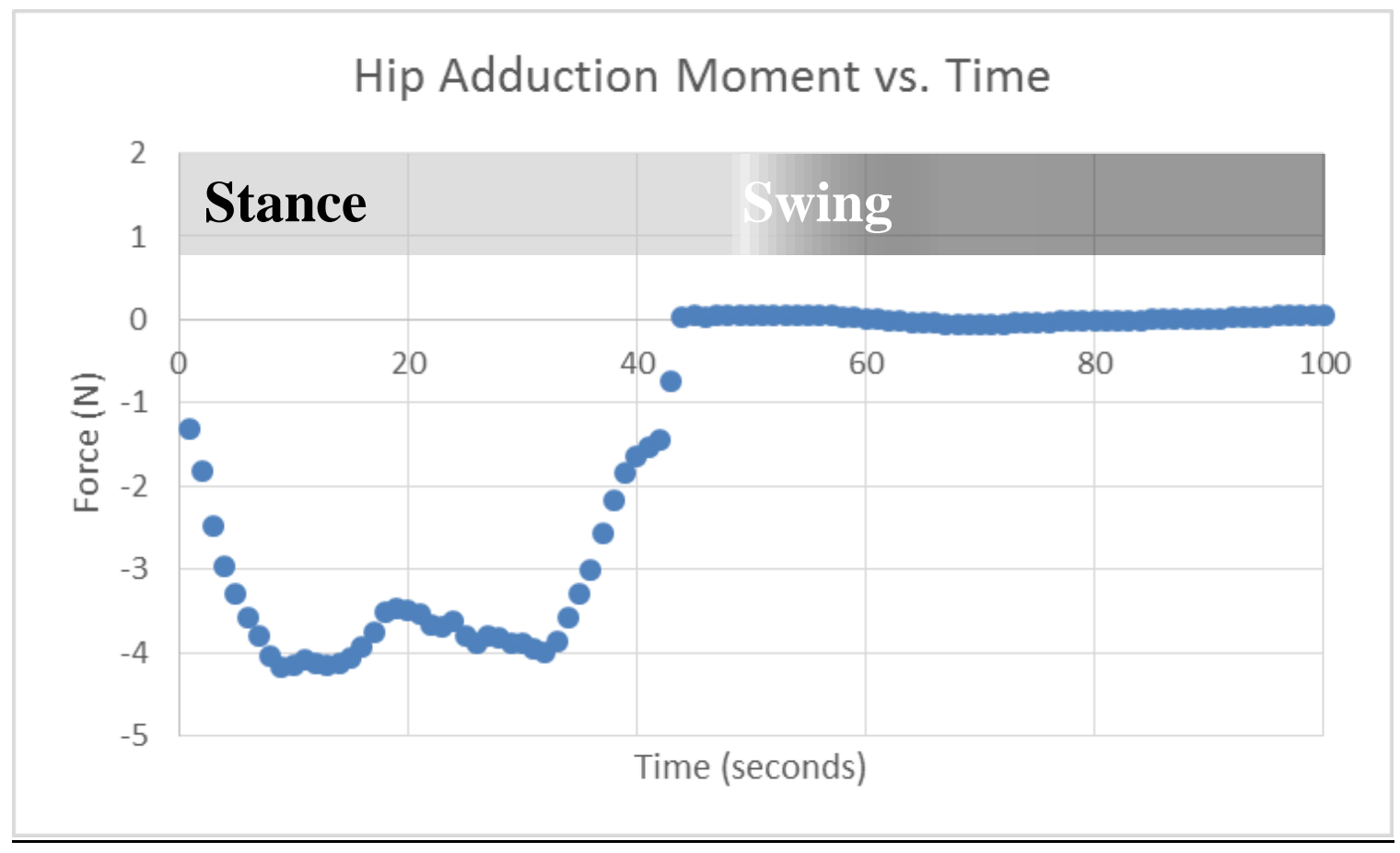

FIGURE 50 - Hip adduction moment during a representative gait phase.

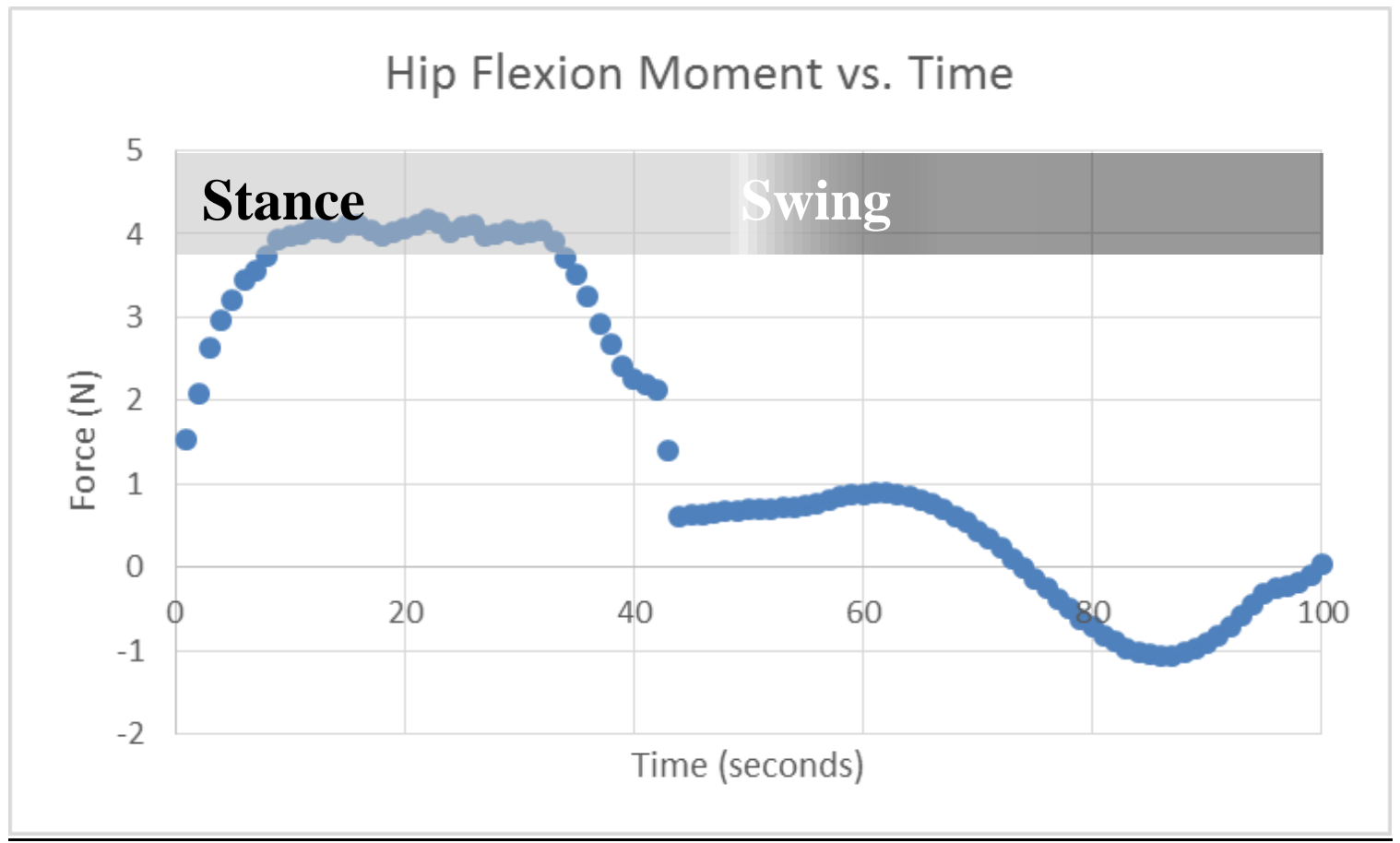

FIGURE 51 - Hip flexion moment during a representative gait phase. 


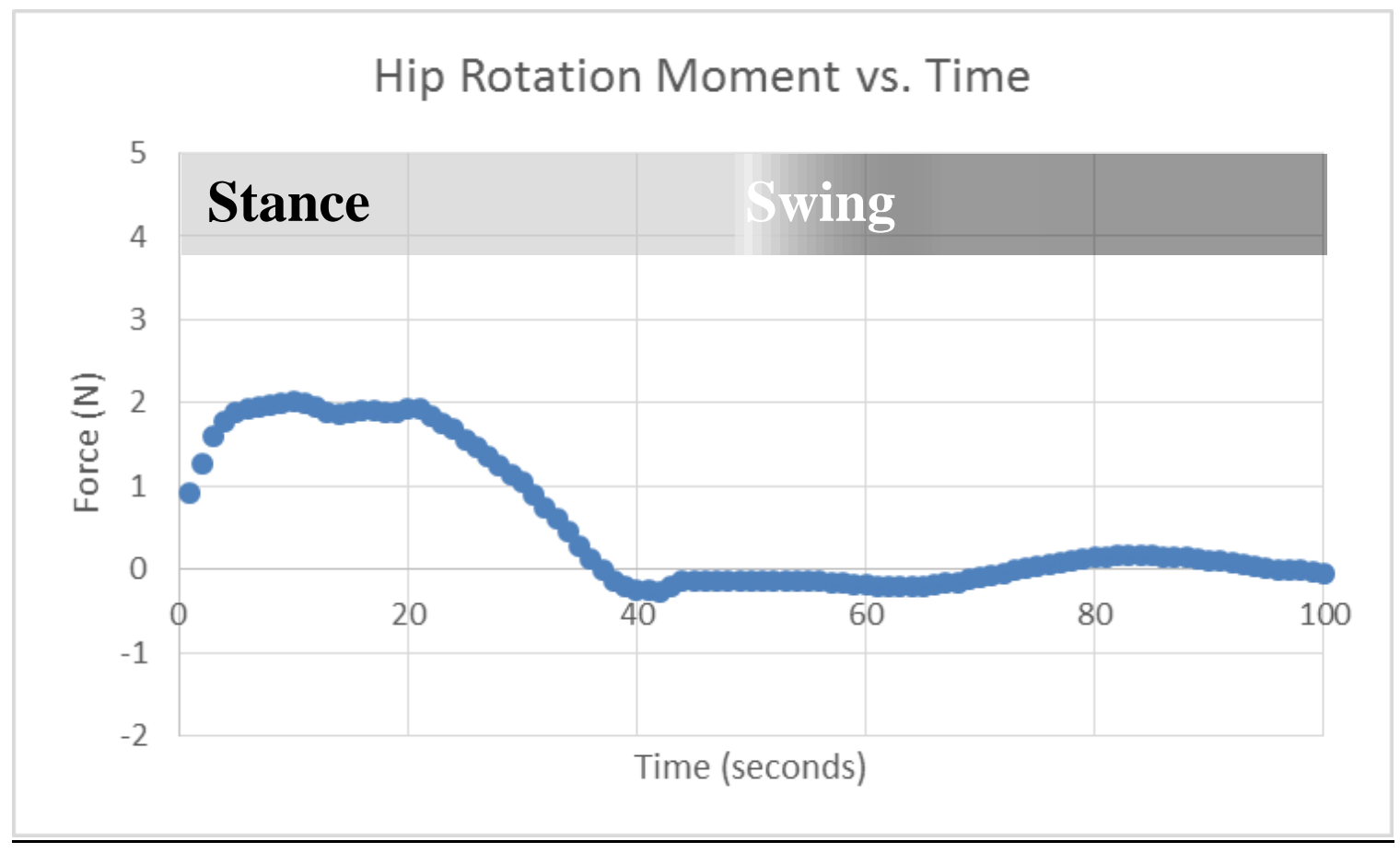

FIGURE 52 - Hip rotation moment during a representative gait phase.

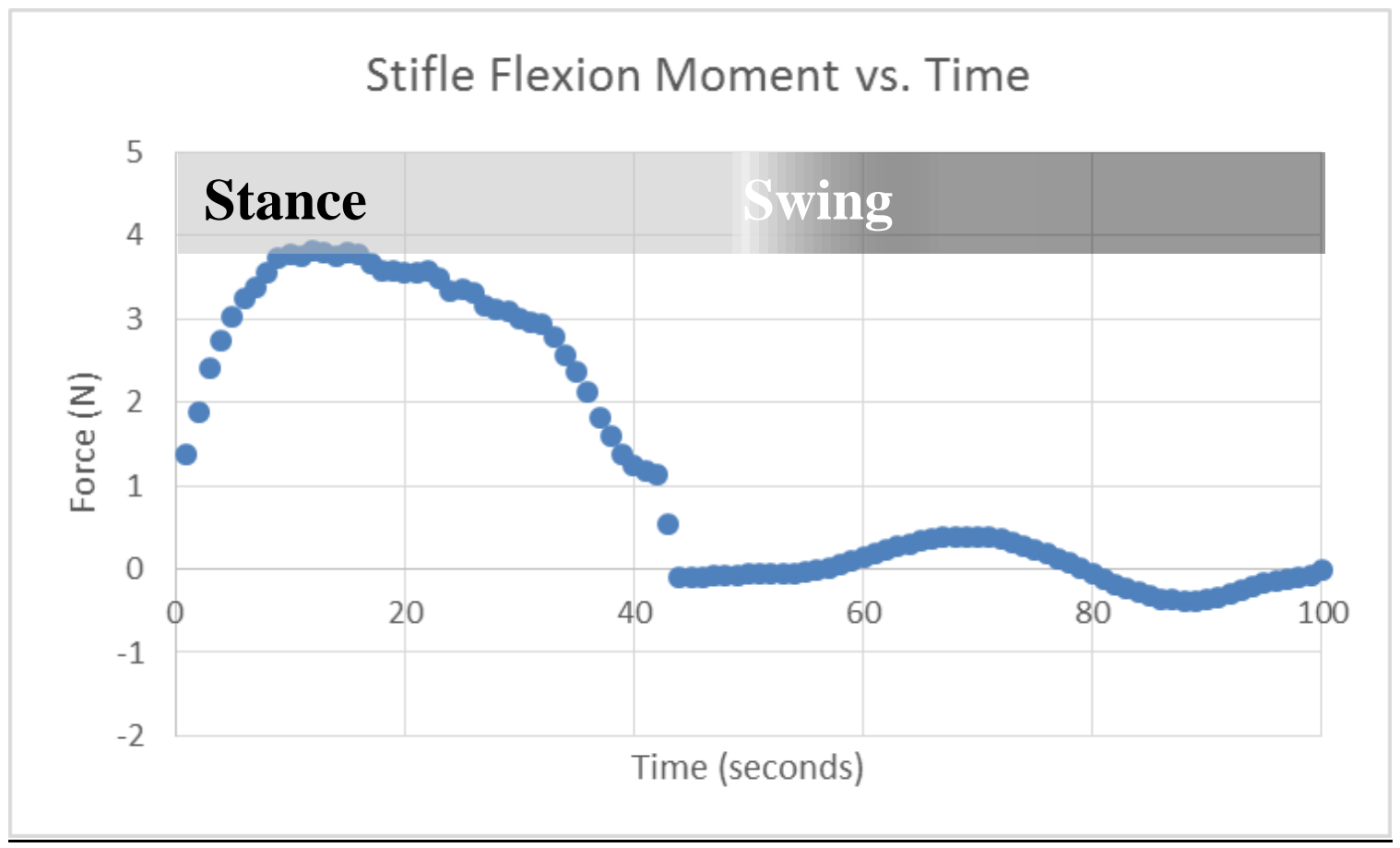

FIGURE 53 - Stifle flexion moment during a representative gait phase. 
April 12, 2016

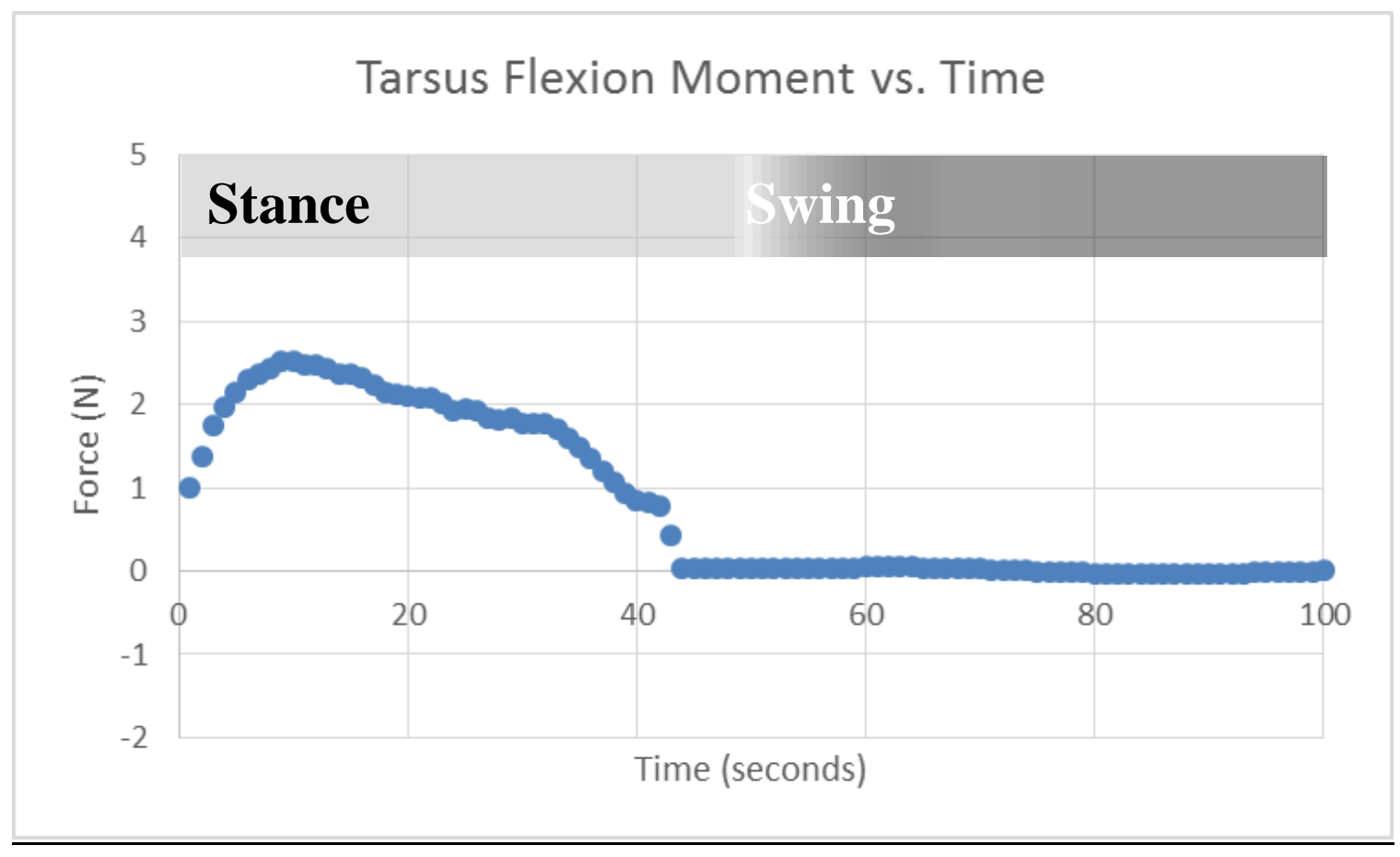

FIGURE 54 - Tarsus flexion moment of the right and left limb during stance and swing. 


\section{DISCUSSION}

\section{A. Model Description}

A validated musculoskeletal model in OpenSim allows researchers to estimate muscle forces/activations and joint torques using kinematic and kinetic data. It can also be used to perform parametric analyses to describe how changes in muscle mass or neurological input to muscles affect individual muscle forces during gait. The model developed in this study is an approximation of musculature and bony structures of the canine pelvis and pelvic limbs, which is capable of estimating joint angles, joint torques, muscle forces, and muscle activation patterns during gait.

Modeled joint kinematics were compared to kinematics calculated using marker trajectory data obtained from motion capture to describe the model's ability to predict kinematic data. Additionally, a parametric sensitivity analysis was performed to estimate muscle activation patterns during walking gait following reductions in maximum muscle isometric force.

\section{$\underline{\text { 1. Joint Kinematics }}$}

Measured joint kinematics were compared to modeled joint kinematics of the hip, stifle and tarsus using peak magnitude, peak timing, and correlation coefficient. Peak magnitude (normalized to the minimum angle of each joint) differed by $96 \%$ in the hip, $5 \%$ in the stifle, and $8 \%$ in the tarsus. Peak timing differed by $9 \%$ in the hip, $6 \%$ in the stifle, and 3\% in the tarsus. Correlation coefficients were 0.99 in the hip, 0.92 in the stifle, and 0.99 in the tarsus. 
Potential causes of differing marker trajectories are related to how each joint was modeled as well as the definition of joint centers of rotation. The hip joint was modeled as a ball and socket joint, while the stifle and tarsus were modeled as pin joints. Ball and socket joints allow for flexion/extension, internal/external rotation and adduction/abduction, while pin joints are constrained to flexion/extension in the sagittal plane. When comparing JCS kinematics to OS predicted kinematics, differences can occur due to joint internal/external rotation and/or adduction/abduction, which cannot be represented in the model-predicted stifle and tarsus kinematics. Additionally, virtual markers are defined as rigidly fixed to a bone, and segments are therefore constrained to movement around the defined joints.

Estimation of the hip joint center has been described in many human studies that propose different approaches (Camomilla et al., 2006; Ehrig et al., 2006; Wu et al., 2002). The method used to estimate hip joint center of location in this study was a geometric fit based on imaging data, where joint center was assumed to be the center of a sphere fitted to the femoral head. A limitation of this method was that CT imaging was conducted with the subject in one position, therefore it is possible that hip joint center of rotation may change with changes in hip flexion. Despite this limitation, the femoral head as center of rotation has been used as a standard in human subjects with radiographs in standard anatomical position (Boudriot et al., 2006; Bouffard et al., 2012).

The center of rotation of the stifle joint was defined as the location between medial and lateral femoral condyle; this approximation was based on definitions from a study which also used a rigid body model of a canine pelvis and pelvic limb (Headrick et al., 2014). Although a reasonable approximation, it has been demonstrated that the 
location of the center of rotation of the stifle varies as a function of flexion/extension in dogs (Ireland et al., 1986). Human models of the knee have been developed to address this limitation. For example, a recent study included the design of an OpenSim model of the human knee which incorporates mediolateral, proximodistal, and anteroposterior translation (Xu et al., 2015) as a function of knee flexion/extension angle.

The center of rotation of the tarsus joint was defined as the location between medial and lateral malleolus; this approximation was based on definitions from a study which also used a rigid body model of a canine pelvis and pelvic limb (Headrick et al., 2014). To the author's knowledge, no studies have been done to model abduction/adduction or internal/external rotation in the tarsal joint of dogs.

Discrepancy between measured and modeled kinematics can also be due to skin/fur movement. Markers in this study were attached to subjects using double-sided tape, without any removal of fur. Additionally, marker locations where the bony prominence is covered by muscle (such as the greater trochanter and ischiatic tuberosity) are particularly prone to skin movement.

It is also important to note that medial markers of the femur and tibia could not be tracked in this study (due to the small stature of the Dachshund pelvic limb and limitations in camera placement). Because of this limitation, flexion/extension is defined for the tarsus joint as rotation around an axis normal to the vectors formed by the tibial crest/lateral malleolus and calcaneus/distal $5^{\text {th }}$ metatarsal. Therefore, the reported flexion/extension angles of the tarsus and stifle are a composite measurement of joint angle, and cannot differentiate between internal/external rotation and flexion/extension. That is, rotation into or out of the sagittal plane can influence stifle flexion/extension. 
Despite limitations in joint representation, definitions of joint centers of rotation differences in the origin of coordinate systems, and skin movement artifact, correlation coefficients of all markers are $>0.85$, indicating that the measured and virtual marker trajectories have a similar profile.

\section{Residual Forces and Joint Torques}

General guidelines for OpenSim models suggest a peak residual force should not exceed 10-20 N (Hicks, 2012). Although peak residual force in the canine model is within the recommended range $(13.5 \mathrm{~N})$, it is important to note that the mass in the canine model is a fraction of that seen in human models (average Dachshund weight in this study was $5.7 \mathrm{~kg}$, compared to reported averages of healthy male humans of $76.4 \mathrm{~kg}$ (Giakas \& Baltzopoulos, 1997)).

Residual force analysis showed a consistent pattern in the vertical direction where peak residual force occurs between $25-35 \%$ stance. The residual force corresponds closely with measured ground reaction force (Figure 55), suggesting that the additional force is being applied to the center of the pelvis to counteract the ground reaction force.

Unaccounted force is largely due to the exclusion of the torso and thoracic limbs in the model. This result is expected in an incomplete model. 


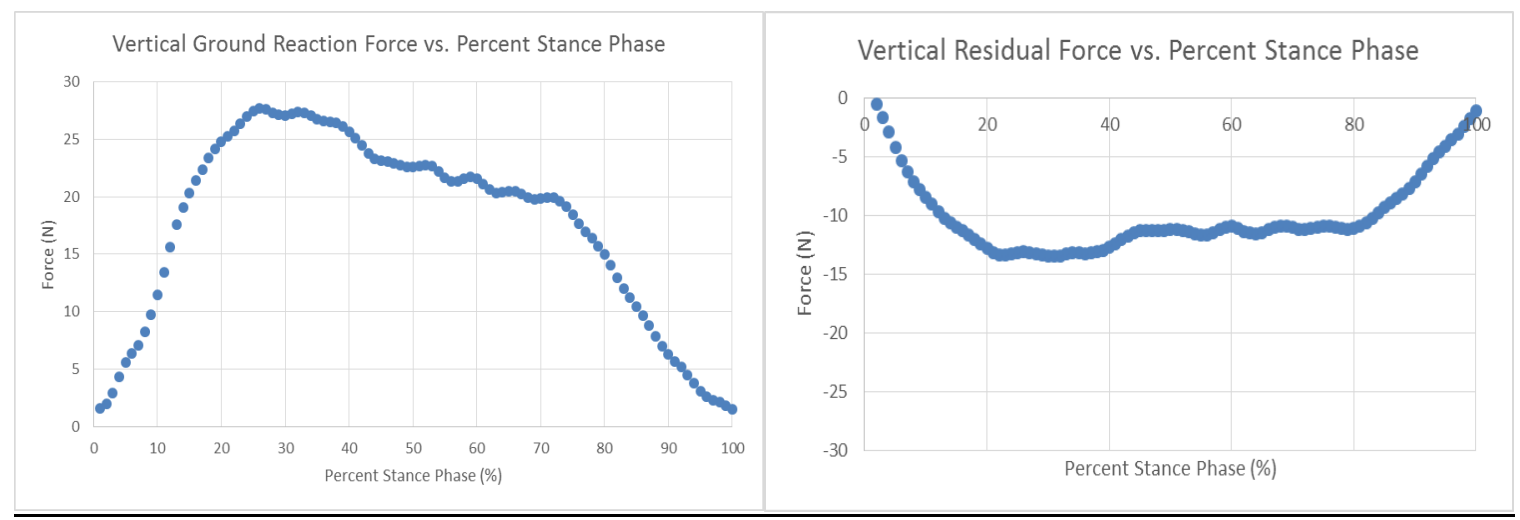

FIGURE 55 - Mean residual vertical force of the pelvis (left) and mean vertical ground reaction force (right).

Peak joint torque were higher during stance than during swing. Peak joint torques of the hip, stifle, and tarsus are shown below in Table XI.

TABLE XI

PEAK HIP, STIFLE AND TARSUS JOINT TORQUES IN HEALTHY SUBJECT 1. REPORTED VALUES ARE IN NM.

\begin{tabular}{|l|c|c|c|c|c|}
\hline & Hip Flexion & $\begin{array}{c}\text { Hip } \\
\text { Adduction }\end{array}$ & Hip Rotation & Stifle Flexion & $\begin{array}{c}\text { Tarsus } \\
\text { Flexion }\end{array}$ \\
\hline Stance & 4.2 & 4.2 & 2 & 3.8 & 2.5 \\
\hline Swing & 1.1 & 0.1 & 0.2 & 0.4 & 0 \\
\hline
\end{tabular}

Ideally, joint torques from the model could be compared to joint torques calculated using inverse dynamics of a Dachshund. To the author's knowledge, no study has been done to calculate joint torques in a Dachshund pelvic limb; doing so was outside the scope of this study. However, results can be compared to inverse dynamic studies of other breeds of dog.

Results of two recent studies report joint torques in the pelvic limb of Labrador Retrievers (Colborne et al., 2005; Ragetly et al., 2010). Values of peak joint moments from these studies are shown below in Table XII. 
TABLE XII

PEAK JOINT MOMENTS FOR HIP, STIFLE AND TARSUS FROM VARIOUS

CANINE STUDIES, REPORTED IN NM/KG. ASTERISKS REPRESENT VALUES

THAT WERE NOT REPORTED OR CALCULATED.

\begin{tabular}{|l|c|c|c|c|c|c|}
\hline & $\begin{array}{c}\text { Hip } \\
\text { Stance }\end{array}$ & $\begin{array}{c}\text { Hip } \\
\text { Swing }\end{array}$ & $\begin{array}{c}\text { Stifle } \\
\text { Stance }\end{array}$ & $\begin{array}{c}\text { Stifle } \\
\text { Swing }\end{array}$ & $\begin{array}{c}\text { Tarsus } \\
\text { Stance }\end{array}$ & $\begin{array}{c}\text { Tarsus } \\
\text { Swing }\end{array}$ \\
\hline Colborne $^{\mathbf{1}}$ & $*$ & $*$ & 0.2 & $*$ & 0.6 & $*$ \\
\hline Ragetly $^{\mathbf{2}}$ & 0.5 & 0.2 & 0.4 & 0.1 & 0.25 & $\sim 0$ \\
\hline $\begin{array}{l}\text { Current } \\
\text { Study }\end{array}$ & 0.8 & 0.2 & 0.7 & 0.1 & 0.5 & $\sim 0$ \\
\hline
\end{tabular}

${ }^{1}$ - Colborne et al., 2005; ${ }^{2}$ - Ragetly et al., 2010

Values reported in both the Ragetly and Colborne study are reported as moment/body mass. To compare these values to joint torques reported here, peak joint torques of the left limb were averaged and divided by the mass of healthy subject $1(5.35 \mathrm{~kg})$.

Normalized values from this study are very similar to values reported from Ragetly et al, with the largest difference being the stifle during stance. However, additional kinematic trials should be used as input to the OpenSim model to further characterize joint torques in a Dachshund during gait.

The study from Colborne does not provide a complete picture of joint torque (values are reported only for the stifle and tarsal joints during stance). Additionally, kinematic and kinetic data used in this study was obtained in dogs during trot (average velocity was $1.98 \mathrm{~m} / \mathrm{s}$ in the Colborne study compared to $0.59 \mathrm{~m} / \mathrm{s}$ in this study). Despite these differences, it is clear that the magnitude of joint torque seen in this study is reasonable for a dog of the weight of a Dachshund during swing. However, during stance model-predicted joint torques of the hip, stifle, and tarsus are almost double the reported 
values from Ragetly et al. Tarsus stance compares favorably to values reported from Colborne et al., however it is important to note that dogs in the Colborne study were trotting at over three times the speed reported in the representative trial of this study. Determining joint torques via inverse dynamics based on kinematic and kinetic data of a Dachshund during gait is recommended as future verification of the model.

\section{Muscle Activation Patterns}

Muscle activation patterns of healthy subject 1 during walking gait were predicted using the model at baseline maximum isometric force. (Outcomes from this analysis are shown in results, sub-section D.)

\section{a. Muscle activation patterns}

A parametric analysis to determine the influence of maximum isometric force on muscle activation patterns was conducted. The gait cycle was simulated with 10, 20, 30 and $40 \%$ reductions in maximum isometric force across all muscles. Visually, it appears that a reduction of maximum isometric force has little effect on individual muscle activation patterns, although peak values of activation are affected.

Additionally, some muscle activations during stance demonstrate a ceiling effect where muscles are fully activated for long periods during stance (Figure 56). This ceiling effect is present in the adductor magnus, cranial tibial, iliacus, long digital extensor, quadratus femoris, rectus femoris, Sartorius, tensor fascia lata, and vastus groups. It should be noted that the quadriceps group all demonstrated the ceiling effect during stance; this is potentially caused by the high joint moment seen in the stifle during stance $(0.7 \mathrm{~N} * \mathrm{~m} / \mathrm{kg})$. 


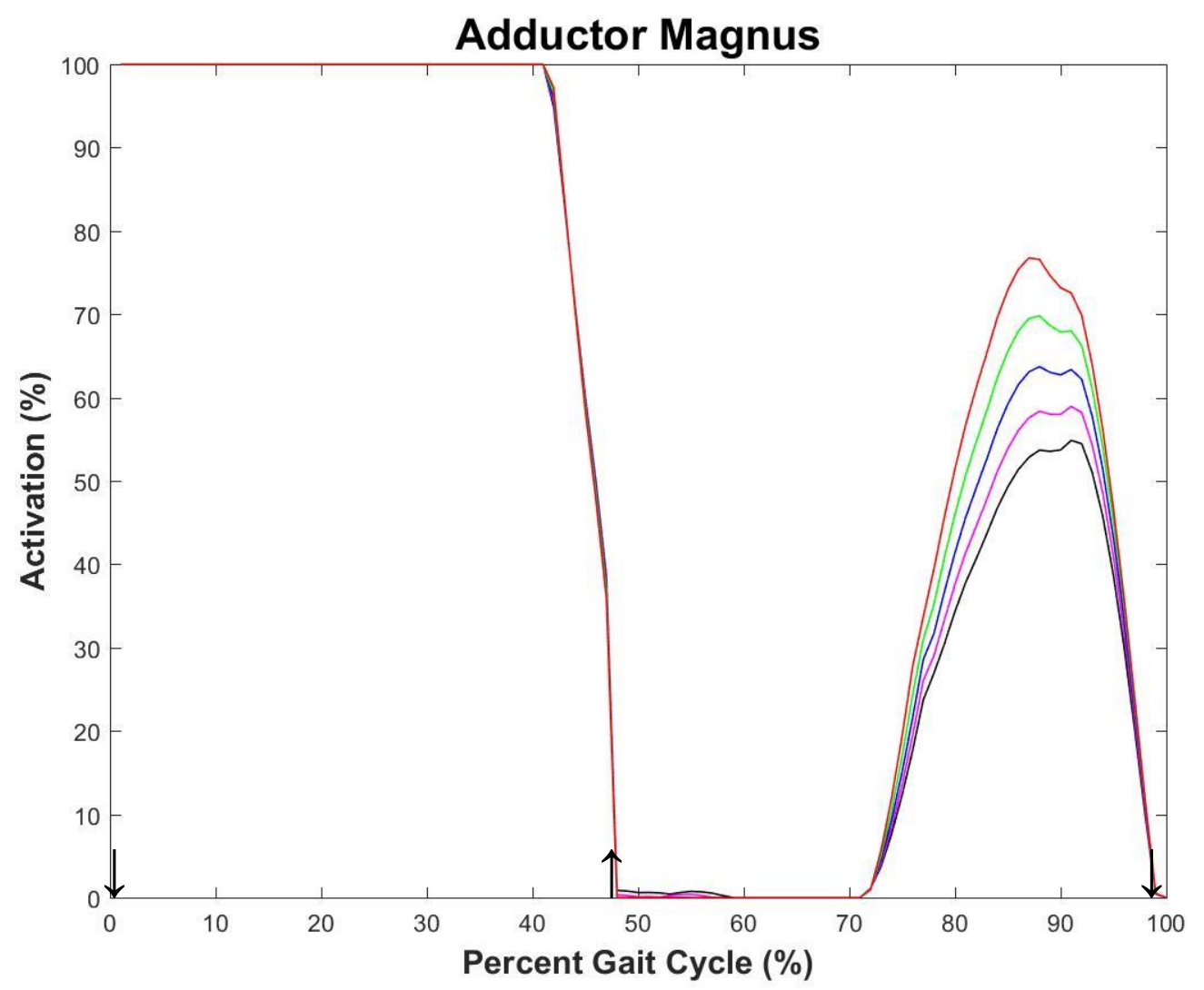

FIGURE 56 - Muscle activation of the adductor magnus. The ceiling effect occurred during the stance phase.

Ideally, simulated muscle activation is compared to EMG recordings of the subject. EMG could not be obtained for this study, therefore a direct comparison cannot be made. However, a comparison can be made to muscle activation patterns reported in the scientific literature. A study of muscle activation patterns during walking gait was performed by Wentink using fine wire EMG on 22 muscles in the canine pelvic limb (Wentink, 1976). EMG patterns were described during stance and swing, and are compared to the muscle activation patterns from this study (Figure 57). In the model, the extensor digitorum longus, tibialis cranialis, adductor, vastus lateralis/medialis, rectus 
femoris, and tensor fascia lata were primarily active during stance. The vastus lateralis/medialis, rectus femoris, tensor fascia lata, sartorius and gluteus medius were active during the first half of swing, while the adductor, semimembranosus, semitendinosus, and biceps femoris were active during the second half of swing. This result is expected based on kinematic data, where hip flexion occurs earlier in swing and is driven by hip flexors (tensor fascia lata and gluteus medius), while stifle extension occurs earlier in swing and is driven by stifle extensors (vastus lateralis/medialis and rectus femoris). Stifle flexion in the latter half of stance is driven by action of the semimembranosus, semitendinosus, and biceps femoris. Although these patterns intuitively make sense, further trials can help verify these results.

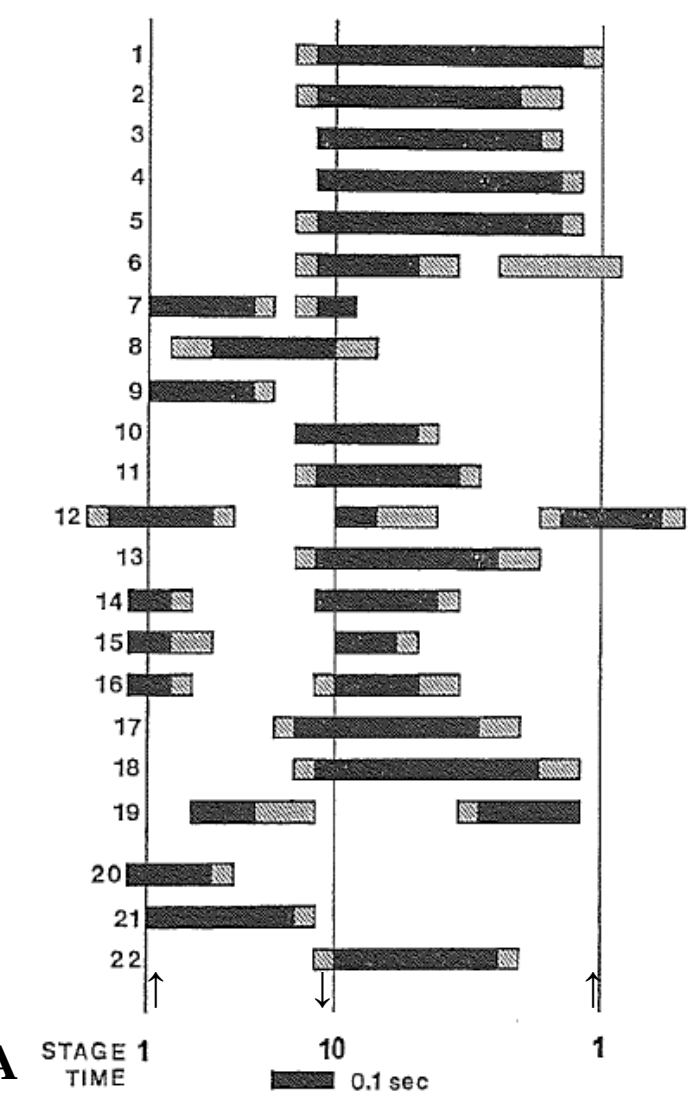




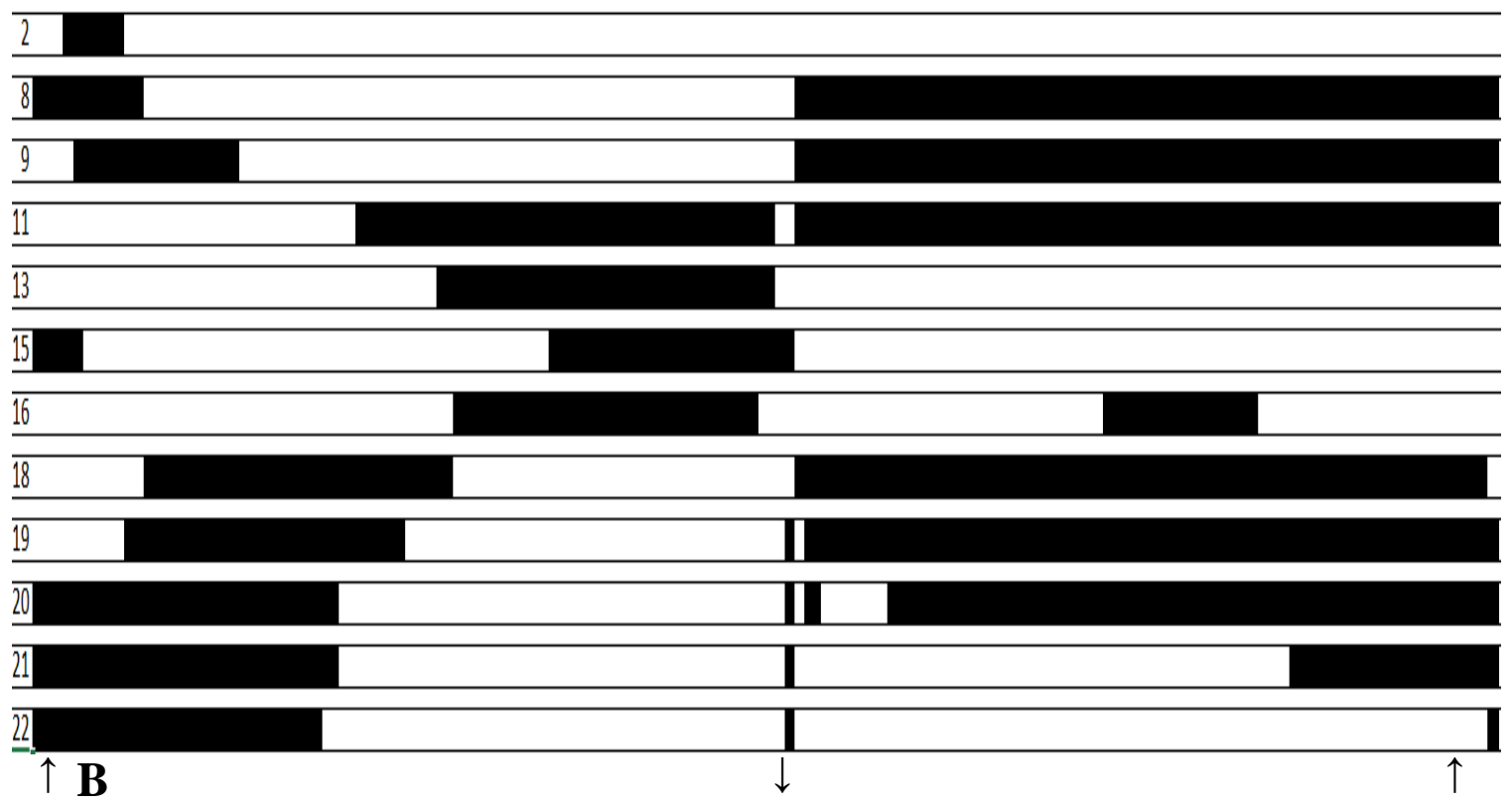

FIGURE 57 - A) Muscle activation patterns reported from (Wentink, 1976) and B) muscle activation patterns in this study. Muscles correspond to numbers as follows: 1. Interosseous (not in model) 2. Gastrocnemius medialis 3. Gastrocnemius lateralis (not in model) 4. Flexor digitorum superficialis (not in model) 5. Hallucis longus (not in model) 6. Popliteus (not in model) 7. Peroneus longus (not in model) 8. Extensor digitorum longus 9. Tibialis cranialis 10. Gracilis (not in model) 11. Adductor 12. Pectineus (not in model) 13. Semimembranosus (cranial) 14. Semimembranosus (caudal) (not in model) 15. Semitendinosus 16. Biceps femoris (caudal) 17. Biceps femoris (cranial) (not in model) 18. Vastus lateralis/medialis 19. Rectus femoris 20. Tensor fascia lata 21.

Sartorius 22. Gluteus medius

Another feature of the model is muscles that are more active throughout both phases of gait. This is particularly noticeable in the adductor magnus (muscle 11, Figure 57) and gluteus medius (muscle 22, Figure 57) which are both active almost entirely throughout the stance and swing phases. Both of these muscles are expected to be active 
during early stance, when the majority of the ground reaction force is applied. This could be caused by these muscles assuming the load of smaller supporting muscles, which are not included in this model. Small muscles that could not be clearly distinguished using CT data were not included in this model (such as the halluces longus, popliteus, peroneus longus, and pectineus).

There are several limitations in this muscle activation pattern comparison. Patterns from the literature are for five Greyhounds (mean age not reported) (Wentink, 1976). The study does not indicate mass of the dogs, although the average mass of greyhounds ranges from 27-40 kg. The differences in gait between Dachshunds and Greyhounds is not extensively studied, but it is visually obvious that these breeds of dogs are morphologically different and have differing kinematics during gait. Additionally, average walking speed of the Greyhounds in the Wentink study was $1.25 \mathrm{~m} / \mathrm{s}$, compared to $0.81 \mathrm{~m} / \mathrm{s}$ in this study. These factors can affect the timing and magnitude of muscle activation, therefore it is impossible to draw deeper conclusions from this comparison (Colborne et al., 2006; Goslow et al., 1981). A study measuring muscle activity levels in Dachshunds during walking gait could provide a more useful comparison, and is recommended to further validate the model.

A dissection study of multiple Dachshunds could provide muscle attachment points of small muscles not included in the model, as well as more accurate descriptions of attachment points of muscles with broad attachments. It should also be noted that fiber lengths and tendon slack lengths were obtained from literature studies or approximated for this study which could affect activation patterns; a dissection study could also 
investigate these parameters to ensure the model is as physiologically similar to a Dachshund as possible.

\section{B. Parametric Sensitivity Analysis}

Injuries that prevent muscle use for prolonged periods of time (such as spinal cord injury) can lead to muscle atrophy, which affects a muscles ability to produce force. The amount of force a muscle can produce is described in OpenSim as "maximum isometric force", which effectively provides a limit on the amount of force a muscle can produce. The goal of the parametric sensitivity analysis was to describe how changes in maximum isometric force affect peak muscle activation. Muscle activation sensitivity to maximum isometric force was assessed by reducing maximum isometric force of all muscles by a fixed percentage and performing a static optimization analysis for each new model across all trials.

Peak muscle activation was affected by changes in maximum isometric force. In general, the effect was amplified for higher reductions of maximum isometric force; sensitivity indices tended to increase with increasing reductions in maximum isometric force. During stance, only the biceps femoris, gemellus, Sartorius, and semitendinosus were highly affected (peak absolute sensitivity index $>1$ ). During swing the biceps femoris, cranial tibial, gastrocnemius, quadratus femoris, semimembranosus, and semitendinosus were somewhat sensitive to maximum isometric force (sensitivity indices $<1)$.

Sensitivity indices appear to be influenced by joint torques. This relation between sensitivity index and joint torques is best shown in the differences between stance and swing phase. The ceiling effect is only present during stance, when joint torque is four 
times higher or more than during swing. The ceiling effect described in section 5.3.1 causes sensitivity index to remain unchanged for the adductor magnus, cranial tibial, iliacus, long digital extensor, quadratus femoris, rectus femoris, Sartorius, tensor fascia lata, and vastus group of the left limb. Muscle activation is $100 \%$ at baseline activation, and does not decrease with decreasing maximum isometric force.

During swing, some patterns between muscle groups are present. Hip flexors (adductor magnus, biceps femoris, middle gluteal, semimembranosus, and semitendinosus) all show an increase in activation when maximum isometric force is reduced. Stifle flexors are also affected, but the rectus femoris shows an increase in activation while the vastus group shows a decrease in activation as maximum isometric muscle force is reduced. It is interesting to note that the rectus femoris also has a role in hip flexion. Tarsus flexors (cranial tibial) and tarsus extensors (long digital extensor, gastrocnemius) all showed decreased activation with decreasing maximum isometric force during stance.

Uniform reduction of maximum isometric force has an effect on peak muscle activation, although it appears to have limited effect on the timing of muscle activation. Higher joint torques during the stance phase suggests that differences in joint torques could have an effect on how muscles respond to a change in maximum isometric force. Higher joint torques generally result in decreased sensitivity, because muscles are already fully activated. To model compensation of muscle groups, a non-uniform reduction in maximum isometric force or maximum muscle activation could be applied to the model (i.e. a reduction of a group of muscles which are innervated below a level of spinal cord injury). 


\section{LIMITATIONS}

Several limitations exist within this study and in relation to a musculoskeletal model of the canine pelvic limb in a healthy population.

- Medial markers were not included during motion tracking.

Markers located on the medial aspects of long bones could not be tracked due to obstruction from other limbs. As a result, some bony segments were only defined using two markers (femur and metatarsals). Each segment was therefore defined as a vector, instead of a three-dimensional coordinate system. Defining the relative movement of two bodies using vectors limits the analysis to rotation in one plane, and ignores the effects of abduction/adduction and internal/external rotation. Additionally, the third marker on the tibia/fibula (fibular head) is not located along or parallel to the stifle joint axis of rotation, and therefore the vector representation used to describe the tibia can lead to inaccuracies in stifle joint angle predictions.

- Imaging data from one Dachshund was used to create the model, and is therefore not generalizable to all Dachshunds.

- Not all pelvic limb muscles were included.

16 muscles in each limb. Some of the smaller muscles were not modeled due to limitations in computed tomography resolution. Small muscles that could not be distinguished were excluded from the model.

- Muscles are represented as lines from origin to insertion, with minimal wrapping included.

Muscle path was described using computed tomography of one subject in a supine position used to obtain CT imaging.Points were added to prevent muscles from passing 
through bone. Additionally, muscles with broad attachments to bone (e.g. gluteus) were modeled as attaching at a single point. Muscle paths can be made more anatomically accurate by describing wrapping as it occurs during motion; this can be measured from imaging data with joints positioned at various angles. Muscles with complex geometries have been modeled in OpenSim using multiple muscle paths, which may be a more accurate representation of these muscles (Arnold et al., 2010).

\section{- Joint limitations}

The stifle joint only included one degree of freedom in rotation (flexion/ extension), with no ability to translate. The tarsus joint only had one degree of freedom as well. Joints were assumed to be frictionless.

- Muscle activation dynamics are based on a Hill-type model (Hill, 1938; Zajac, 1989)

As mentioned in section 2.4.2.2, Hill type muscle models are based on measurements of maximum isometric force, optimal fiber length, tendon slack length, and pennation angle. Muscles are represented as a single fiber in this model, which simplifies activation dynamics. Additionally, these muscle models are dependent on the accuracy of optimal fiber length and tendon slack length. Optimal fiber length and pennation angle in this study was scaled based on reported values of different breeds of dog (Shahar \& Milgram, 2001; Williams et al., 2008); these values are therefore neither breed nor subject specific. Furthermore, tendon slack length was assumed to be zero when the subject was at rest in a supine position. Tendon slack length has been validated against joint moment curves in humans (Delp et al., 1990), however joint moment curves have not been obtained in dogs and thus were not available for the current study. Finally, multi-muscle activation in this 
model is determined by using a least squares approach to minimize the amount of activation produced to generate force. This methodology may not accurately represent the physiological strategy used in dogs. It should also be noted that when developing a model of spinal cord injured dogs, this activation strategy may not be applicable.

- The torso and thoracic limbs were not included in the model.

The model described in this paper is a partial model, however it is important to note that residual force applied to the pelvis in the model accounts for the missing forces in the model. This simplification allows for the collection of ground reaction force on only the pelvic limbs, which is necessary given that force plates can only distinguish one force at a time.

- EMG was not obtained, therefore muscle activation could not be verified.

Dogs in this study were client owned, and therefore fine wire-EMG was not a feasible option. Surface EMG - the other alternative - provides an overall picture of muscle activation and cannot isolate specific muscles. 


\section{RECOMMENDATIONS}

- Improve marker locations.

Placing medial markers on the femur, tibia, and tarsus would allow for calculation of adduction and rotation of the hip, stifle, and tarsus. Addition of these markers would help constrain modeled kinematics to a more relevant joint trajectory. Additionally, future kinematic data recording should take efforts to reduce motion artifact (such as shaving the locations where markers are attached to the dog).

- Magnetic resonance imaging

Computed tomography was used to define muscle parameters in this study, although this method of imaging has limitations. Magnetic resonance imaging could be using in addition to computed tomography for descriptions of small muscle parameters and more accurate description of muscle path in larger muscles that attach via tendons. If this method is used, the subject should be positioned to obtain multiple joint angles to improve the descriptions of muscle path in the OpenSim model.

- A dissection study of multiple Dachshunds could provide more accurate fiber lengths and descriptions of attachment points of muscles with broad attachments.

- Tendon slack length

Tendon slack length could be estimated in several Dachshunds, and incorporated into the model. Human OpenSim models have based tendon slack length on joint moment curves measured in multiple subjects (Delp et al., 1990). To the author's knowledge, no studies have measured passive joint moment in Dachshunds. Therefore a study to measure passive moments would need to be performed before adjustments in tendon slack length can be made. 


\section{CONCLUSIONS}

The goals of this study were to develop a musculoskeletal model of a Dachshund pelvis and pelvic limb that could be used to simulate gait. The OpenSim musculoskeletal model developed in this study was a first approximation of a Dachshund pelvis and pelvic limb that was used to simulate gait, and to the authors knowledge is the first model which incorporates both joint and musculature descriptions. Anatomically accurate data obtained from a computed tomography scan was combined with kinematic and kinetic data to create the model. The model represented a $5.35 \mathrm{~kg}$ Dachshund, and was used to estimate joint kinematics, joint torque, and multi-muscle activation patterns during both the stance and swing phase of gait. Muscle maximum isometric force was reduced by a fixed percentage to represent four scenarios that can be used to evaluate the effects of muscle atrophy on peak muscle activation and activation patterns. Maximum isometric force affected peak activation, although the timing of activation appeared to be largely unaffected. Verification of the model was performed by comparing measured joint kinematics to simulated kinematics by calculating correlation coefficients of joint kinematics as well as measured and virtual marker trajectories.

The model-predicted kinematics were found to correlate well to the measured kinematics. This suggests that this musculoskeletal model was able to reasonably represent the kinematics of a Dachshund during walking gait.

Residual force in the model was consistently $\sim 50 \%$ body weight in the vertical direction, which appears to be compensating for undefined anatomy and mass in the model (torso and thoracic limbs). Residual force in an incomplete model is necessary to account for segments that are not modeled. Further investigation is necessary to 
determine how to distribute this residual force. An independent inverse dynamic analysis of the data would allow for further investigation of residual force and moments.

Recording muscle activation patterns using EMG would further verify the accuracy of modeled activations, however it should be noted that recording EMG in Dachshunds presents a challenge for several reasons. The population of Dachshunds recruited in this study are client owned; owners are unlikely to consent to the use of finewire EMG. It is difficult to record the activity of individual muscles in a Dachshund using surface EMG due to their small size. Additionally, surface EMG limits marker placement during motion capture. These markers also provide unwanted sensory information to the dogs, which is an uncontrolled factor that can potentially affect gait.

The model described herein includes the mass and inertial properties of a Dachshund hindlimb, muscle force production capabilities, and muscle origin/insertion locations. This model allows for prediction of multi-muscle activation patterns in Dachshunds without the limitations of EMG. Additionally, it provides a quantitative means to evaluate therapeutic interventions and enables researchers to investigate the influence of various parameters on muscle activation patterns. Ideally, this model can provide a tool to allow future studies to investigate the effects of neuromuscular conditions on muscle activation patterns.

Limitations of this model include a one degree of freedom stifle model, the exclusion of the torso, and non-species specific muscle properties (tendon slack length, optimal fiber length), and provide a foundation and path for creating a more physiologically relevant model. 


\section{References}

Anderson, F. C., \& Pandy, M. G. (1999). A Dynamic Optimization Solution for Vertical Jumping in Three Dimensions. Comput Methods Biomech Biomed Engin, 2(3), 201-231. doi:10.1080/10255849908907988

Arnold, E. M., Ward, S. R., Lieber, R. L., \& Delp, S. L. (2010). A model of the lower limb for analysis of human movement. Ann Biomed Eng, 38(2), 269-279. doi:10.1007/s10439-009-9852-5

Basso, D. M., Beattie, M. S., \& Bresnahan, J. C. (1995). A sensitive and reliable locomotor rating scale for open field testing in rats. $J$ Neurotrauma, 12(1), 1-21. Retrieved from http://www.ncbi.nlm.nih.gov/pubmed/7783230

Bergknut, N., Egenvall, A., Hagman, R., Gustas, P., Hazewinkel, H. A., Meij, B. P., \& Lagerstedt, A. S. (2012). Incidence of intervertebral disk degeneration-related diseases and associated mortality rates in dogs. J Am Vet Med Assoc, 240(11), 1300-1309. doi:10.2460/javma.240.11.1300

Boudriot, U., Hilgert, J., \& Hinrichs, F. (2006). Determination of the rotational center of the hip. Arch Orthop Trauma Surg, 126(6), 417-420. doi:10.1007/s00402-0060157-y

Bouffard, V., Begon, M., Champagne, A., Farhadnia, P., Vendittoli, P. A., Lavigne, M., \& Prince, F. (2012). Hip joint center localisation: A biomechanical application to hip arthroplasty population. World J Orthop, 3(8), 131-136. doi:10.5312/wjo.v3.i8.131

Braund, K. G. (1994). Clinical Syndromes in Veterinary Neurology: Mosby, Incorporated.

Brown, L. M., Lopez, J. R., Olsen, J. A., Rudel, R., Simmons, R. M., Taylor, S. R., \& Wanek, L. A. (1982). Branched skeletal muscle fibers not associated with dysfunction. Muscle Nerve, 5(8), 645-653. doi:10.1002/mus.880050810

Camomilla, V., Cereatti, A., Vannozzi, G., \& Cappozzo, A. (2006). An optimized protocol for hip joint centre determination using the functional method. $J$ Biomech, 39(6), 1096-1106. doi:10.1016/j.jbiomech.2005.02.008

Coffey, N., Harrison, A. J., Donoghue, O. A., \& Hayes, K. (2011). Common functional principal components analysis: a new approach to analyzing human movement data. Hum Mov Sci, 30(6), 1144-1166. doi:10.1016/j.humov.2010.11.005

Colborne, G. R., Innes, J. F., Comerford, E. J., Owen, M. R., \& Fuller, C. J. (2005). Distribution of power across the hind limb joints in Labrador Retrievers and Greyhounds. Am J Vet Res, 66(9), 1563-1571. Retrieved from http://www.ncbi.nlm.nih.gov/pubmed/16261830

Colborne, G. R., Walker, A. M., Tattersall, A. J., \& Fuller, C. J. (2006). Effect of trotting velocity on work patterns of the hind limbs of Greyhounds. Am J Vet Res, 67(8), 1293-1298. doi:10.2460/ajvr.67.8.1293

Daffertshofer, A., Lamoth, C. J., Meijer, O. G., \& Beek, P. J. (2004). PCA in studying coordination and variability: a tutorial. Clin Biomech (Bristol, Avon), 19(4), 415428. doi:10.1016/j.clinbiomech.2004.01.005 
Delp, S. L., Anderson, F. C., Arnold, A. S., Loan, P., Habib, A., John, C. T., . . Thelen, D. G. (2007). OpenSim: Open-Source Software to Create and Analyze Dynamic Simulations of Movement. IEEE Transactions on Biomedical Engineering, 54(11), 1940-1950. doi:10.1109/TBME.2007.901024

Delp, S. L., Loan, J. P., Hoy, M. G., Zajac, F. E., Topp, E. L., \& Rosen, J. M. (1990). An interactive graphics-based model of the lower extremity to study orthopaedic surgical procedures. Biomedical Engineering, IEEE Transactions on, 37(8), $757-$ 767. Retrieved from http://ieeexplore.ieee.org/xpls/abs_all.jsp?arnumber $=102791$

Dona, G., Preatoni, E., Cobelli, C., Rodano, R., \& Harrison, A. J. (2009). Application of functional principal component analysis in race walking: an emerging methodology. Sports Biomech, 8(4), 284-301. doi:10.1080/14763140903414425

Donoghue, O. A., Harrison, A. J., Coffey, N., \& Hayes, K. (2008). Functional data analysis of running kinematics in chronic Achilles tendon injury. Med Sci Sports Exerc, 40(7), 1323-1335. doi:10.1249/MSS.0b013e31816c4807

Duhamel, A., Devos, P., Bourriez, J. L., Preda, C., Defebvre, L., \& Beuscart, R. (2006). Functional data analysis for gait curves study in Parkinson's disease. Stud Health Technol Inform, 124, 569-574. Retrieved from http://www.ncbi.nlm.nih.gov/pubmed/17108578

Earle-Bridges, D. C. C. M. (2004). The Dachshund Handbook: Barron's Educational Series.

Ehrig, R. M., Taylor, W. R., Duda, G. N., \& Heller, M. O. (2006). A survey of formal methods for determining the centre of rotation of ball joints. J Biomech, 39(15), 2798-2809. doi:10.1016/j.jbiomech.2005.10.002

Erwin, W. M., Islam, D., Inman, R. D., Fehlings, M. G., \& Tsui, F. W. L. (2011). Notochordal cells protect nucleus pulposus cells from degradation and apoptosis: implications for the mechanisms of intervertebral disc degeneration. Arthritis Research \& Therapy, 13(6). doi:10.1186/ar3548

Evans, H. E., \& deLahunta, A. (1988). Guide to the Dissection of the Dog (Sixth ed.). St. Louis: Saunders.

Frankel, H. L., Hancock, D. O., Hyslop, G., Melzak, J., Michaelis, L. S., Ungar, G. H., . . . Walsh, J. J. (1969). The value of postural reduction in the initial management of closed injuries of the spine with paraplegia and tetraplegia. Spinal Cord, 7(3), 179-192. doi:10.1038/sc.1969.30

Fu, Y.-C., Torres, B. T., \& Budsberg, S. C. (2010). Evaluation of a three-dimensional kinematic model for canine gait analysis. American Journal of Veterinary

Research, 71(10), 1118-1122. doi:10.2460/ajvr.71.10.1118

Giakas, G., \& Baltzopoulos, V. (1997). Time and frequency domain analysis of ground reaction forces during walking: an investigation of variability and symmetry. Gait \&amp; Posture, 5(3), 189-197. Retrieved from http://www.gaitposture.com/article/S0966-6362(96)01083-1/abstract

Gong, J. K., Arnold, J. S., \& Cohn, S. H. (1964). Composition of Trabecular and Cortical Bone. Anat Rec, 149, 325-331. Retrieved from http://www.ncbi.nlm.nih.gov/pubmed/14208979

Goslow, G. E., Seeherman, H. J., Taylor, C. R., McCutchin, M. N., \& Heglund, N. C. (1981). Electrical activity and relative length changes of dog limb muscles as a 
function of speed and gait. Journal of Experimental Biology, 94(1), 15-42. Retrieved from http://jeb.biologists.org/content/94/1/15

http://www.ncbi.nlm.nih.gov/pubmed/7310312

files/891/15.html

Grood, E. S., \& Suntay, W. J. (1983). A joint coordinate system for the clinical description of three-dimensional motions: application to the knee. Journal of biomechanical engineering, 105(2), 136-144. Retrieved from http://www.ncbi.nlm.nih.gov/pubmed/6865355

http://biomechanical.asmedigitalcollection.asme.org/article.aspx?articleid=1396188

Hamilton, L., Franklin, R. J., \& Jeffery, N. D. (2008). Quantification of deficits in lateral paw positioning after spinal cord injury in dogs. BMC Vet Res, 4, 47. doi:10.1186/1746-6148-4-47

Hamilton, L., Franklin, R. J. M., \& Jeffery, N. D. (2007). Development of a universal measure of quadrupedal forelimb-hindlimb coordination using digital motion capture and computerised analysis. BMC Neuroscience, 8(1). doi:10.1186/14712202-8-77

Hansen, H.-J. (1951). A pathologic-anatomical interpretation of disc degeneration in dogs. Acta Orthopaedica, 20(4), 280-293. Retrieved from http://informahealthcare.com/doi/pdf/10.3109/17453675108991175

Harrison, A. (2014). Applications of Functional Data Analysis in Sport Biomechanics. Paper presented at the International Conference of Biomechanics in Sports, Johnson City, TN.

Headrick, J. F., Zhang, S., Millard, R. P., Rohrbach, B. W., Weigel, J. P., \& Millis, D. L. (2014). Use of an inverse dynamics method to describe the motion of the canine pelvic limb in three dimensions. Am J Vet Res, 75(6), 544-553. doi:10.2460/ajvr.75.6.544

Hicks, J. L. (2012). OpenSim Documentation: User's Guide.

Hill, A. V. (1938). The Heat of Shortening and the Dynamic Constants of Muscle. Proceedings of the Royal Society of London. Series B, Biological Sciences, 126(843), 136-195.

Ireland, W. P., Rogers, J., \& Myers, R. K. (1986). Location of the instantaneous center of joint rotation in the normal canine stifle. Am J Vet Res, 47(4), 837-840. Retrieved from http://www.ncbi.nlm.nih.gov/pubmed/3963586

Johansson, M., Pipkorn, B., \& Lovsund, P. (2009). Child safety in vehicles: validation of a mathematical model and development of restraint system design guidelines for 3-year-olds through mathematical simulations. Traffic Inj Prev, 10(5), 467-478. doi:10.1080/15389580903149243

Johnson, W. L., Jindrich, D. L., Roy, R. R., \& Reggie Edgerton, V. (2008). A threedimensional model of the rat hindlimb: musculoskeletal geometry and muscle moment arms. J Biomech, 41(3), 610-619. doi:10.1016/j.jbiomech.2007.10.004

Keshner, E. A., Statler, K. D., \& Delp, S. L. (1997). Kinematics of the freely moving head and neck in the alert cat. Exp Brain Res, 115(2), 257-266. Retrieved from http://www.ncbi.nlm.nih.gov/pubmed/9224854

Knarr, B. A., Ramsay, J. W., Buchanan, T. S., Higginson, J. S., \& Binder-Macleod, S. A. (2013). Muscle volume as a predictor of maximum force generating ability in the 
plantar flexors post-stroke. Muscle Nerve, 48(6), 971-976.

doi:10.1002/mus.23835

Lawson, D. (1958). Discussion on Comparison of Disorders of the Intervertebral Disc in Man and Animals. Proceedings of the Royal Society of Medicine, 51(7), 569-576. Retrieved from http://www.ncbi.nlm.nih.gov/pmc/articles/PMC1889656/

http://www.ncbi.nlm.nih.gov/pmc/articles/PMC1889656/pdf/procrsmed00357-0137.pdf Levine, G. J., Levine, J. M., Budke, C. M., Kerwin, S. C., Au, J., Vinayak, A., . . Slater, M. R. (2009). Description and repeatability of a newly developed spinal cord injury scale for dogs. Preventive Veterinary Medicine, 89(1-2), 121-127. doi:10.1016/j.prevetmed.2009.02.016

Levine, J. M., Levine, G. J., Porter, B. F., Topp, K., \& Noble-Haeusslein, L. J. (2011). Naturally Occurring Disk Herniation in Dogs: An Opportunity for Pre-Clinical Spinal Cord Injury Research. Journal of Neurotrauma, 28(4), 675-688. doi:10.1089/neu.2010.1645

Marghitu, D. B., \& Nalluri, P. (1997). An analysis of greyhound gait using wavelets. $J$ Electromyogr Kinesiol, 7(3), 203-212. Retrieved from http://www.ncbi.nlm.nih.gov/pubmed/20719706

http://ac.els-cdn.com/S1050641196000351/1-s2.0-S1050641196000351main.pdf? tid=7f6811a8-db6b-11e3-b1fc00000aab0f02\&acdnat $=1400074229$ 74cc141c74f5bd8fae8fceae6fcbce01

Olby, N., Harris, T., Burr, J., Muñana, K., Sharp, N., \& Keene, B. (2004). Recovery of pelvic limb function in dogs following acute intervertebral disc herniations. Journal of neurotrauma, 21(1), 49-59. Retrieved from http://online.liebertpub.com/doi/abs/10.1089/089771504772695940

http://online.liebertpub.com/doi/pdfplus/10.1089/089771504772695940

Olby, N., Levine, J., Harris, T., Muñana, K., Skeen, T., \& Sharp, N. (2003). Long-term functional outcome of dogs with severe injuries of the thoracolumbar spinal cord: 87 cases (1996-2001). Journal of the American Veterinary Medical Association, 222(6), 762-769. doi:10.2460/javma.2003.222.762

Olby, N. J., De Risio, L., Muñana, K. R., Wosar, M. A., Skeen, T. M., Sharp, N. J., \& Keene, B. W. (2001). Development of a functional scoring system in dogs with acute spinal cord injuries. American journal of veterinary research, 62(10), 16241628. Retrieved from http://www.ncbi.nlm.nih.gov/pubmed/11592330

Powell, P. L., Roy, R. R., Kanim, P., Bello, M. A., \& Edgerton, V. R. (1984). Predictability of skeletal muscle tension from architectural determinations in guinea pig hindlimbs. J Appl Physiol Respir Environ Exerc Physiol, 57(6), 17151721. Retrieved from http://www.ncbi.nlm.nih.gov/pubmed/6511546

Ragetly, C. A., Griffon, D. J., Mostafa, A. A., Thomas, J. E., \& Hsiao-Wecksler, E. T. (2010). Inverse dynamics analysis of the pelvic limbs in Labrador Retrievers with and without cranial cruciate ligament disease. Vet Surg, 39(4), 513-522. doi:10.1111/j.1532-950X.2010.00680.x

Ragetly, C. A., Griffon, D. J., Thomas, J. E., Mostafa, A. A., Schaeffer, D. J., Pijanowski, G. J., \& Hsiao-Wecksler, E. T. (2008). Noninvasive determination of body segment parameters of the hind limb in Labrador Retrievers with and without 
cranial cruciate ligament disease. Am J Vet Res, 69(9), 1188-1196.

doi:10.2460/ajvr.69.9.1188

Ramsay, J. O. H., G; Graves, S. (2009). Functional Data Analysis with R and MATLAB. Ramsay, J. O. S., B.W. (2002). Applied Functional Data Analysis: Methods and Case Studies (Vol. 1). New York, NY: Springer.

Ramsay, J. O. S., B.W. (2005). Functional Data Analysis (Second ed.). New York, NY: Springer.

Risbud, M. V., \& Shapiro, I. M. (2011). Notochordal Cells in the Adult Intervertebral Disc: New Perspective on an Old Question. Critical reviews in eukaryotic gene expression, 21(1), 29-41. Retrieved from http://www.ncbi.nlm.nih.gov/pmc/articles/PMC3187872/

http://www.ncbi.nlm.nih.gov/pmc/articles/PMC3187872/pdf/nihms325605.pdf

Roy, R. R., Meadows, I. D., Baldwin, K. M., \& Edgerton, V. R. (1982). Functional significance of compensatory overloaded rat fast muscle. J Appl Physiol Respir Environ Exerc Physiol, 52(2), 473-478. Retrieved from http://www.ncbi.nlm.nih.gov/pubmed/7061301

Ryan, W., Harrison, A., \& Hayes, K. (2006). Functional data analysis of knee joint kinematics in the vertical jump. Sports Biomech, 5(1), 121-138. doi:10.1080/14763141.2006.9628228

Shahar, R., \& Milgram, J. (2001). Morphometric and anatomic study of the hind limb of a dog. Am J Vet Res, 62(6), 928-933. Retrieved from http://www.ncbi.nlm.nih.gov/pubmed/11400852

Shankar, H., Scarlett, J. A., \& Abram, S. E. (2009). Anatomy and pathophysiology of intervertebral disc disease. Techniques in Regional Anesthesia and Pain Management, 13(2), 67-75. doi:10.1053/j.trap.2009.05.001

Smolders, L. A., Bergknut, N., Grinwis, G. C. M., Hagman, R., Lagerstedt, A.-S., Hazewinkel, H. A. W., . . Meij, B. P. (2013). Intervertebral disc degeneration in the dog. Part 2: Chondrodystrophic and non-chondrodystrophic breeds. The Veterinary Journal, 195(3), 292-299. doi:10.1016/j.tvj1.2012.10.011

Spector, S. A., Gardiner, P. F., Zernicke, R. F., Roy, R. R., \& Edgerton, V. R. (1980). Muscle architecture and force-velocity characteristics of cat soleus and medial gastrocnemius: implications for motor control. J Neurophysiol, 44(5), 951-960. Retrieved from http://www.ncbi.nlm.nih.gov/pubmed/7441324

Thelen, D. G. (2003). Adjustment of muscle mechanics model parameters to simulate dynamic contractions in older adults. J Biomech Eng, 125(1), 70-77. Retrieved from http://www.ncbi.nlm.nih.gov/pubmed/12661198

http://biomechanical.asmedigitalcollection.asme.org/article.aspx ?articleid=1409437

Wentink, G. H. (1976). The action of the hind limb musculature of the dog in walking. Acta Anat (Basel), 96(1), 70-80. Retrieved from http://www.ncbi.nlm.nih.gov/pubmed/973540

Williams, S. B., Wilson, A. M., Rhodes, L., Andrews, J., \& Payne, R. C. (2008). Functional anatomy and muscle moment arms of the pelvic limb of an elite sprinting athlete: the racing greyhound (Canis familiaris). J Anat, 213(4), 361372. doi:10.1111/j.1469-7580.2008.00961.x 
Wu, G., Siegler, S., Allard, P., Kirtley, C., Leardini, A., Rosenbaum, D., . . Terminology Committee of the International Society of, B. (2002). ISB recommendation on definitions of joint coordinate system of various joints for the reporting of human joint motion--part I: ankle, hip, and spine. International Society of Biomechanics. J Biomech, 35(4), 543-548. Retrieved from http://www.ncbi.nlm.nih.gov/pubmed/11934426

http://ac.els-cdn.com/S0021929001002226/1-s2.0-S0021929001002226main.pdf? tid=43f81ae6-ec67-11e5-83d800000aab0f6c\&acdnat=1458236577 43a5bb0e4ca31bf8475baf8079454053

Xiao, M., \& Higginson, J. (2010). Sensitivity of estimated muscle force in forward simulation of normal walking. J Appl Biomech, 26(2), 142-149. Retrieved from http://www.ncbi.nlm.nih.gov/pubmed/20498485

http://www.ncbi.nlm.nih.gov/pmc/articles/PMC2877275/pdf/nihms156785.pdf

Xu, H., Bloswick, D., \& Merryweather, A. (2015). An improved OpenSim gait model with multiple degrees of freedom knee joint and knee ligaments. Comput Methods Biomech Biomed Engin, 18(11), 1217-1224. doi:10.1080/10255842.2014.889689

Zajac, F. E. (1989). Muscle and tendon: properties, models, scaling, and application to biomechanics and motor control. Crit Rev Biomed Eng, 17(4), 359-411. Retrieved from http://www.ncbi.nlm.nih.gov/pubmed/2676342 


\section{Appendix A: Functional Principal Components for Healthy Subject 2}
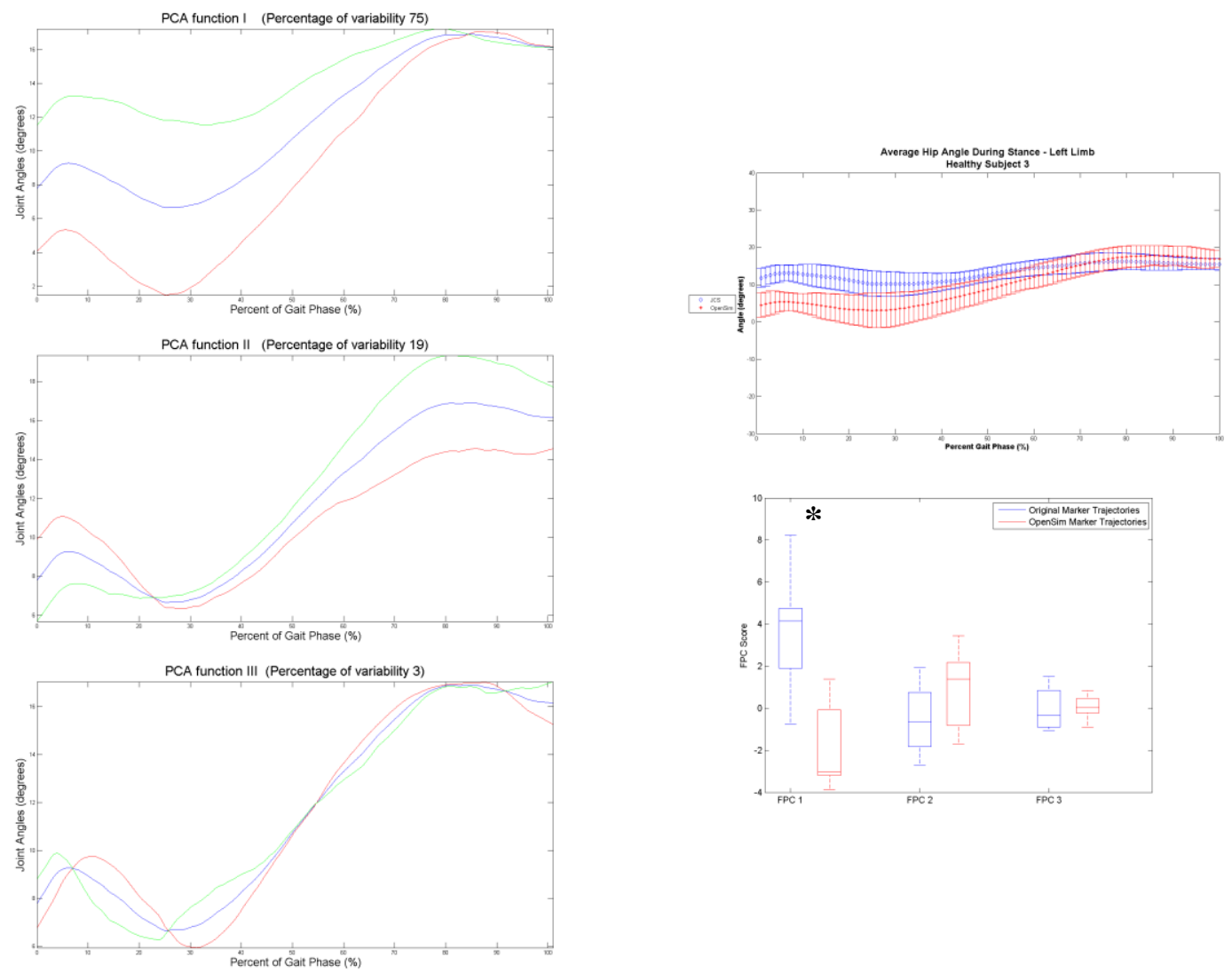

Figure A1. Functional principal component analysis of the second healthy subject for the stance phase of the left hip joint. A-C) Show the mean curve of the OS and JCS curves in blue, plus (green) or minus (red) the first-third principal components, respectively. D) Mean curves of the JCS curves (blue) compared to the OS curves (red) E) Boxplot of the FPC scores for the first three principal components. Significant differences in principal component scores are seen in FPC $1(p<0.001)$. 

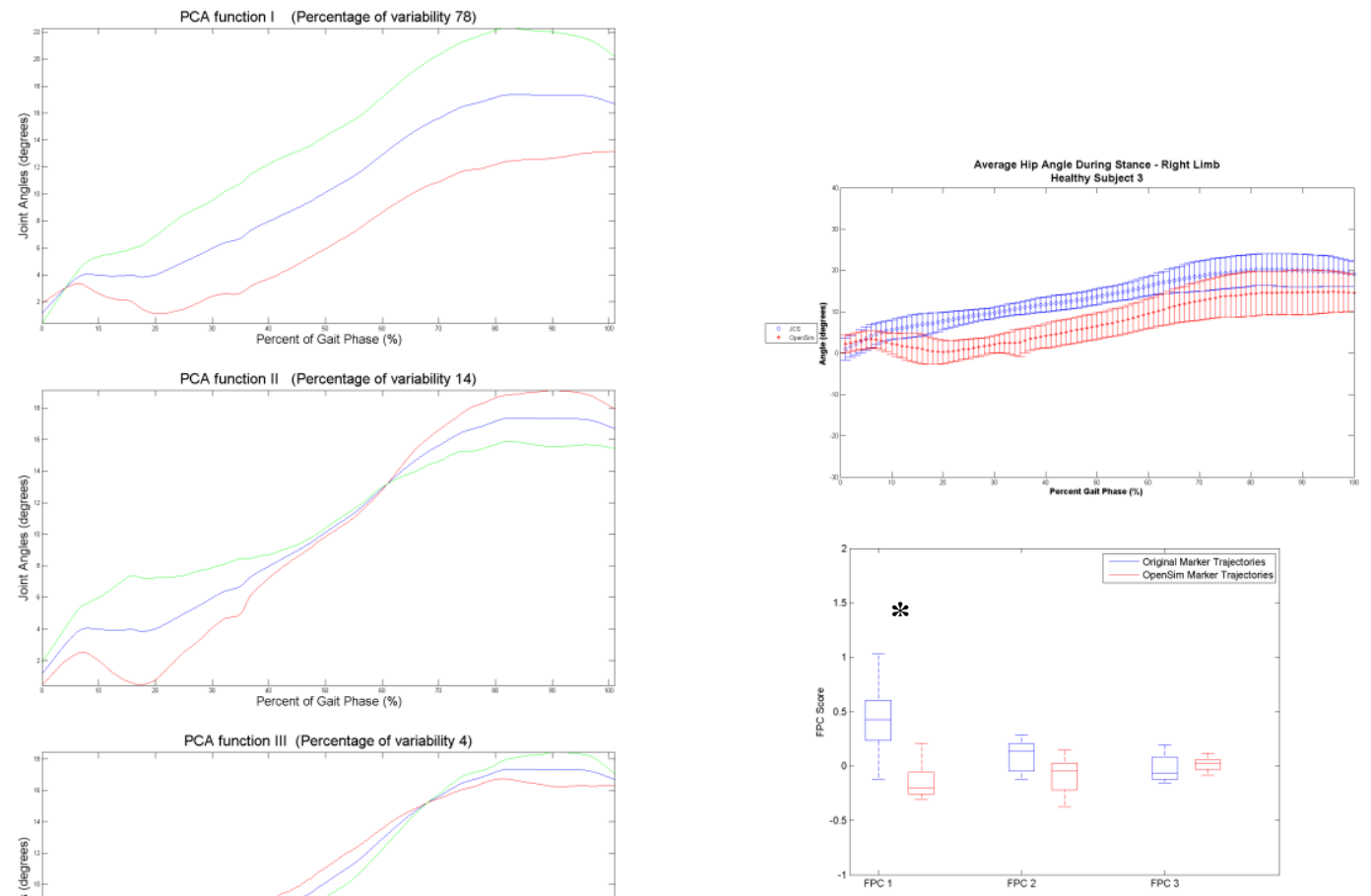

Figure A2. Functional principal component analysis of the second healthy subject for the stance phase of the right hip joint. A-C) Show the mean curve of the OS and JCS curves in blue, plus (green) or minus (red) the first-third principal components, respectively. D) Mean curves of the JCS curves (blue) compared to the OS curves (red) E) Boxplot of the FPC scores for the first three principal components. Significant differences in principal component scores are seen in FPC $1(p<0.001)$. 

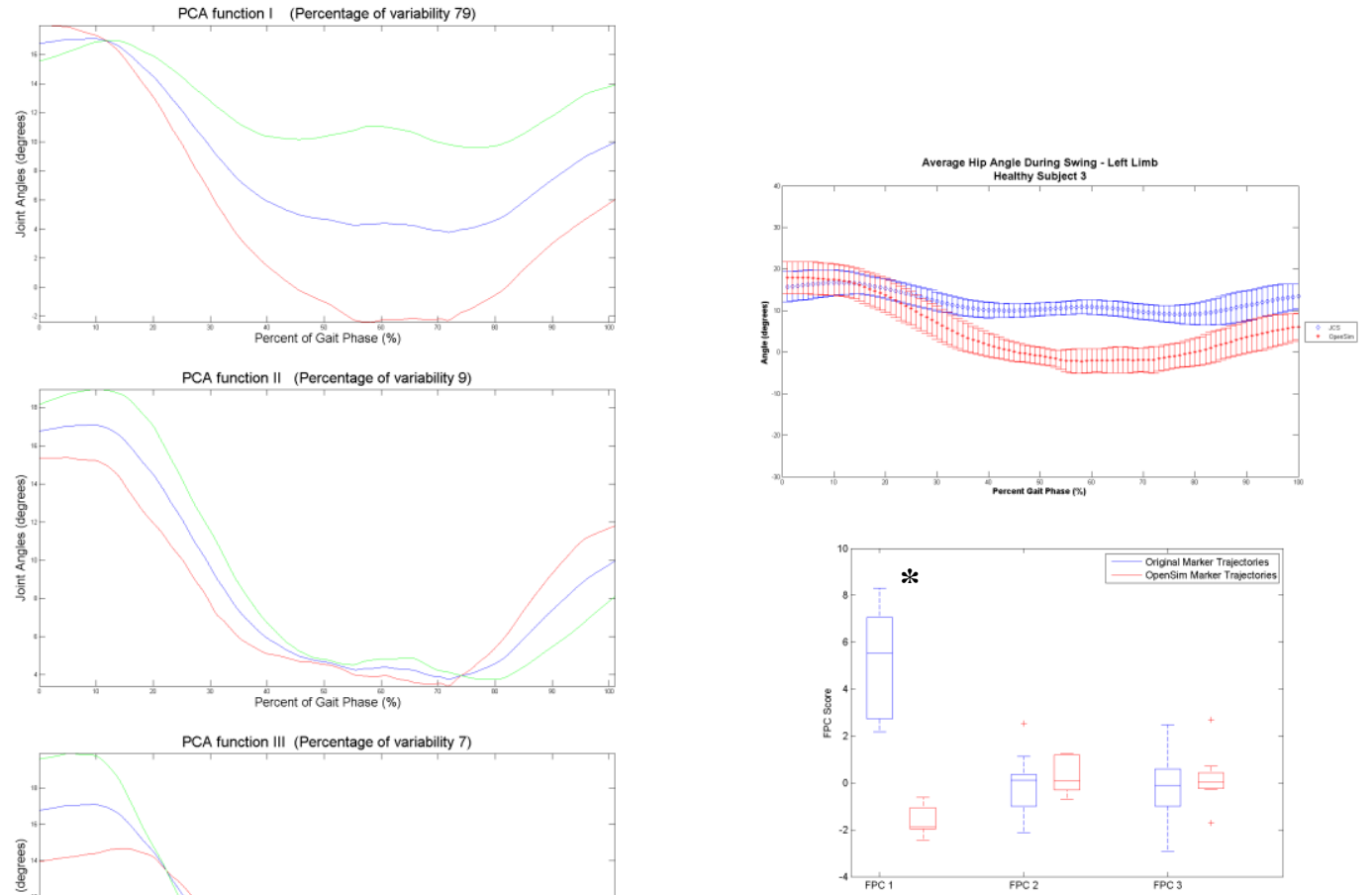

Figure A3. Functional principal component analysis of the second healthy subject for the swing phase of the left hip joint. A-C) Show the mean curve of the OS and JCS curves in blue, plus (green) or minus (red) the first-third principal components, respectively. D) Mean curves of the JCS curves (blue) compared to the OS curves (red) E) Boxplot of the FPC scores for the first three principal components. Significant differences in principal component scores are seen in FPC $1(p<0.001)$. 

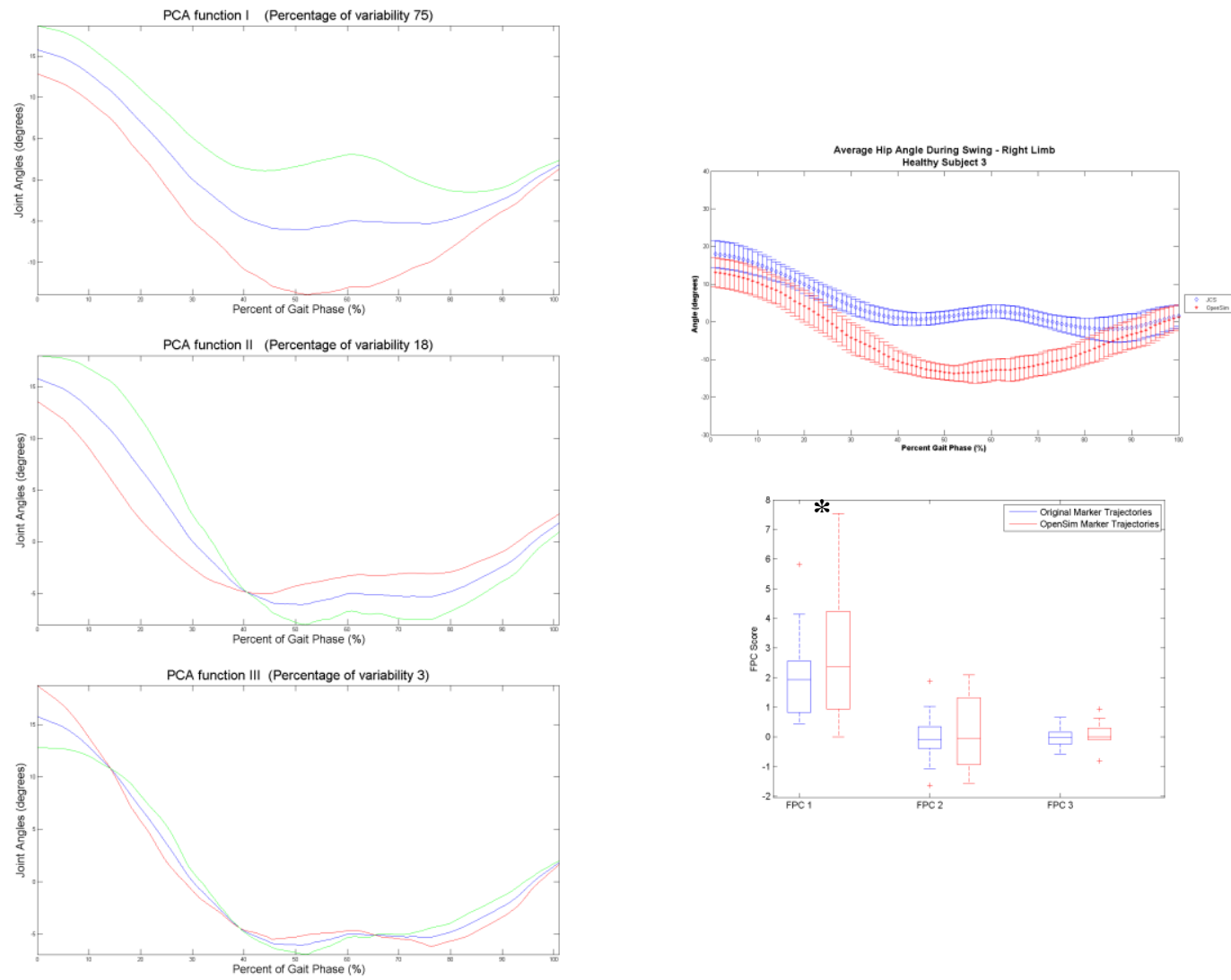

Figure A4. Functional principal component analysis of the second healthy subject for the swing phase of the right hip joint. A-C) Show the mean curve of the OS and JCS curves in blue, plus (green) or minus (red) the first-third principal components, respectively. D) Mean curves of the JCS curves (blue) compared to the OS curves (red) E) Boxplot of the FPC scores for the first three principal components. Significant differences in principal component scores are seen in FPC $1(p=0.001)$. 

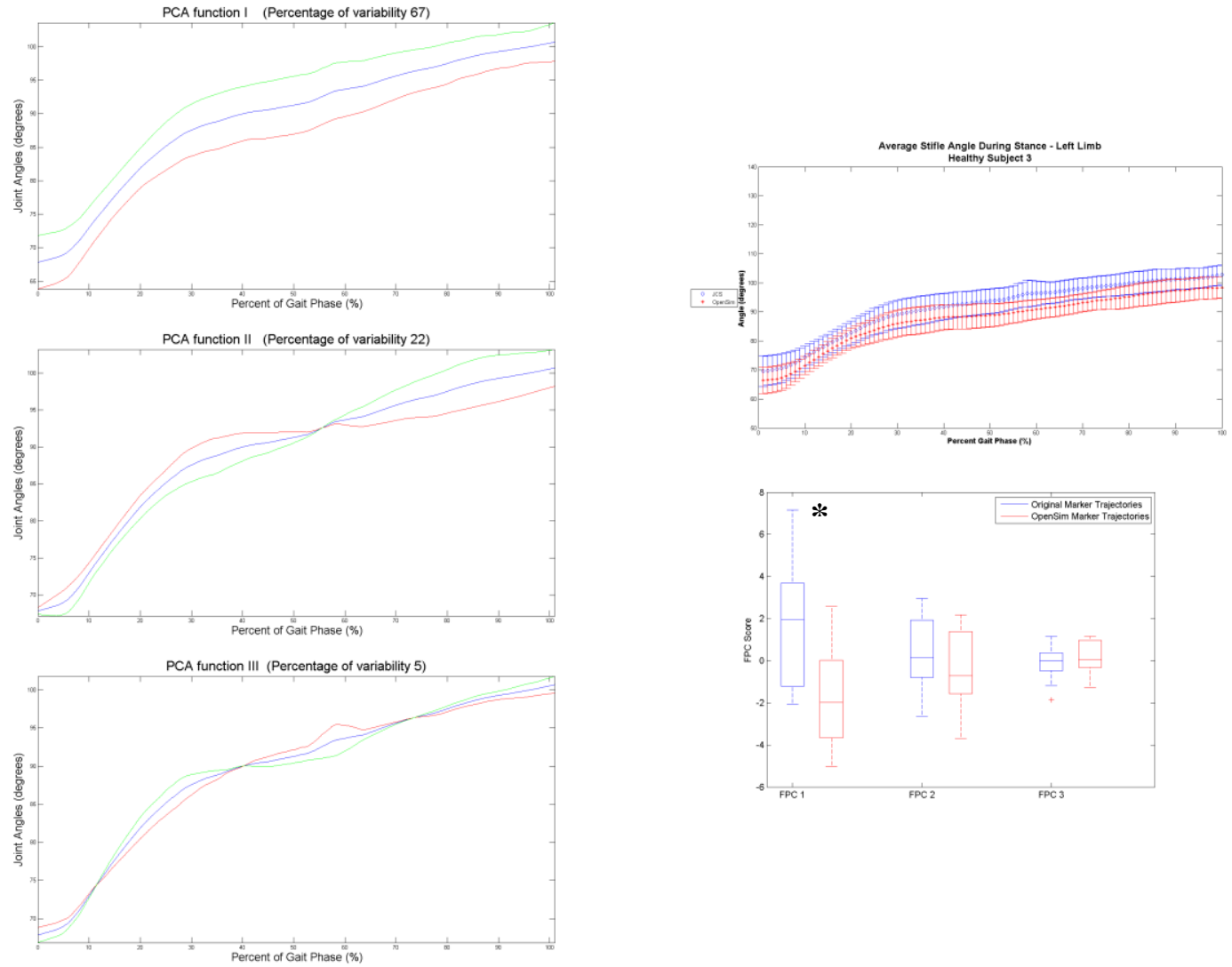

Figure A5. Functional principal component analysis of the second healthy subject for the stance phase of the left stifle joint. A-C) Show the mean curve of the OS and JCS curves in blue, plus (green) or minus (red) the first-third principal components, respectively. D) Mean curves of the JCS curves (blue) compared to the OS curves (red) E) Boxplot of the FPC scores for the first three principal components. Significant differences in principal component scores are seen in FPC $1(p=0.011)$. 

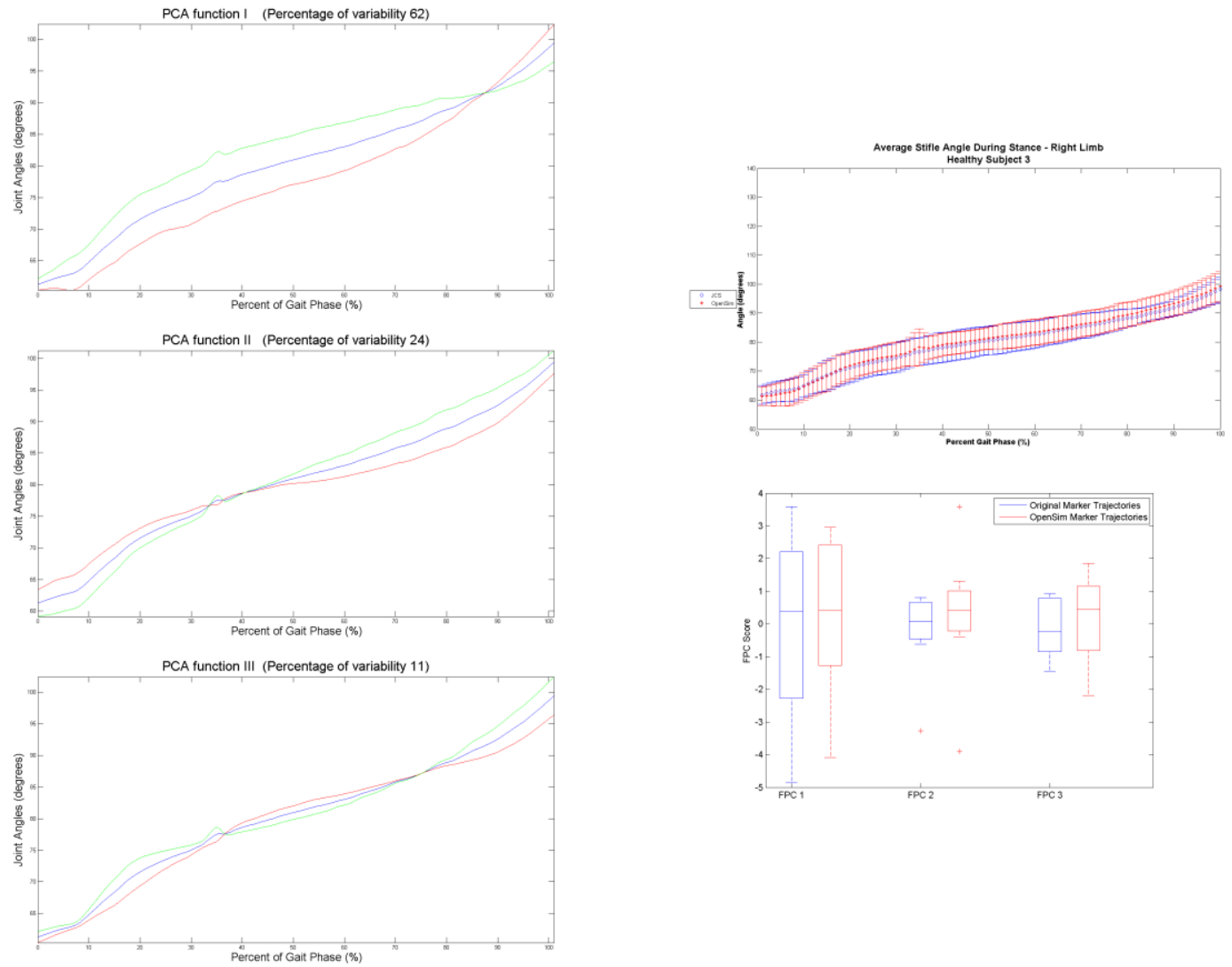

Figure A6. Functional principal component analysis of the second healthy subject for the stance phase of the right stifle joint. A-C) Show the mean curve of the OS and JCS curves in blue, plus (green) or minus (red) the first-third principal components, respectively. D) Mean curves of the JCS curves (blue) compared to the OS curves (red) E) Boxplot of the FPC scores for the first three principal components. 

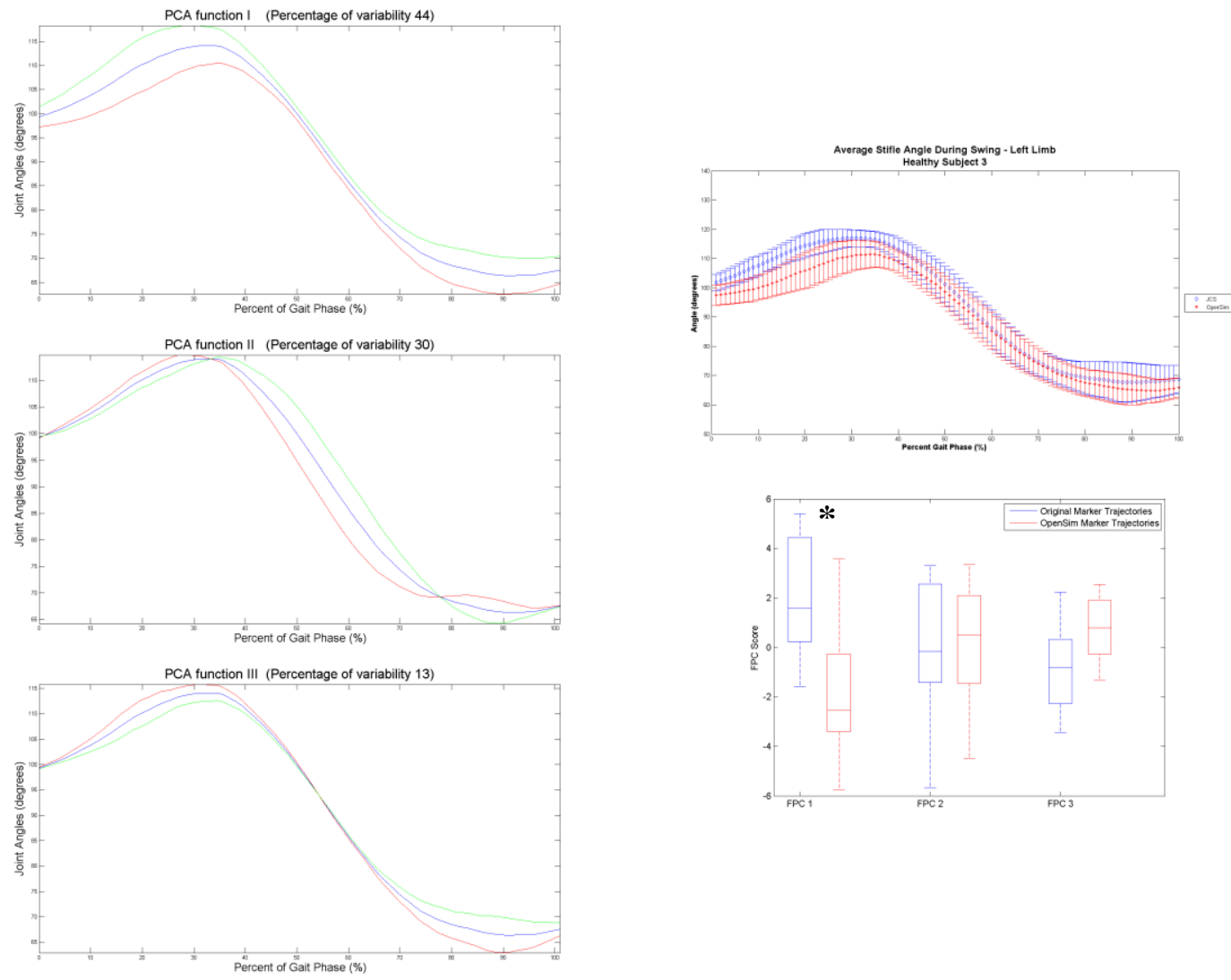

Figure A7. Functional principal component analysis of the second healthy subject for the swing phase of the left stifle joint. A-C) Show the mean curve of the OS and JCS curves in blue, plus (green) or minus (red) the first-third principal components, respectively. D) Mean curves of the JCS curves (blue) compared to the OS curves (red) E) Boxplot of the FPC scores for the first three principal components. Significant differences in principal component scores are seen in FPC $1(p=0.006)$. 

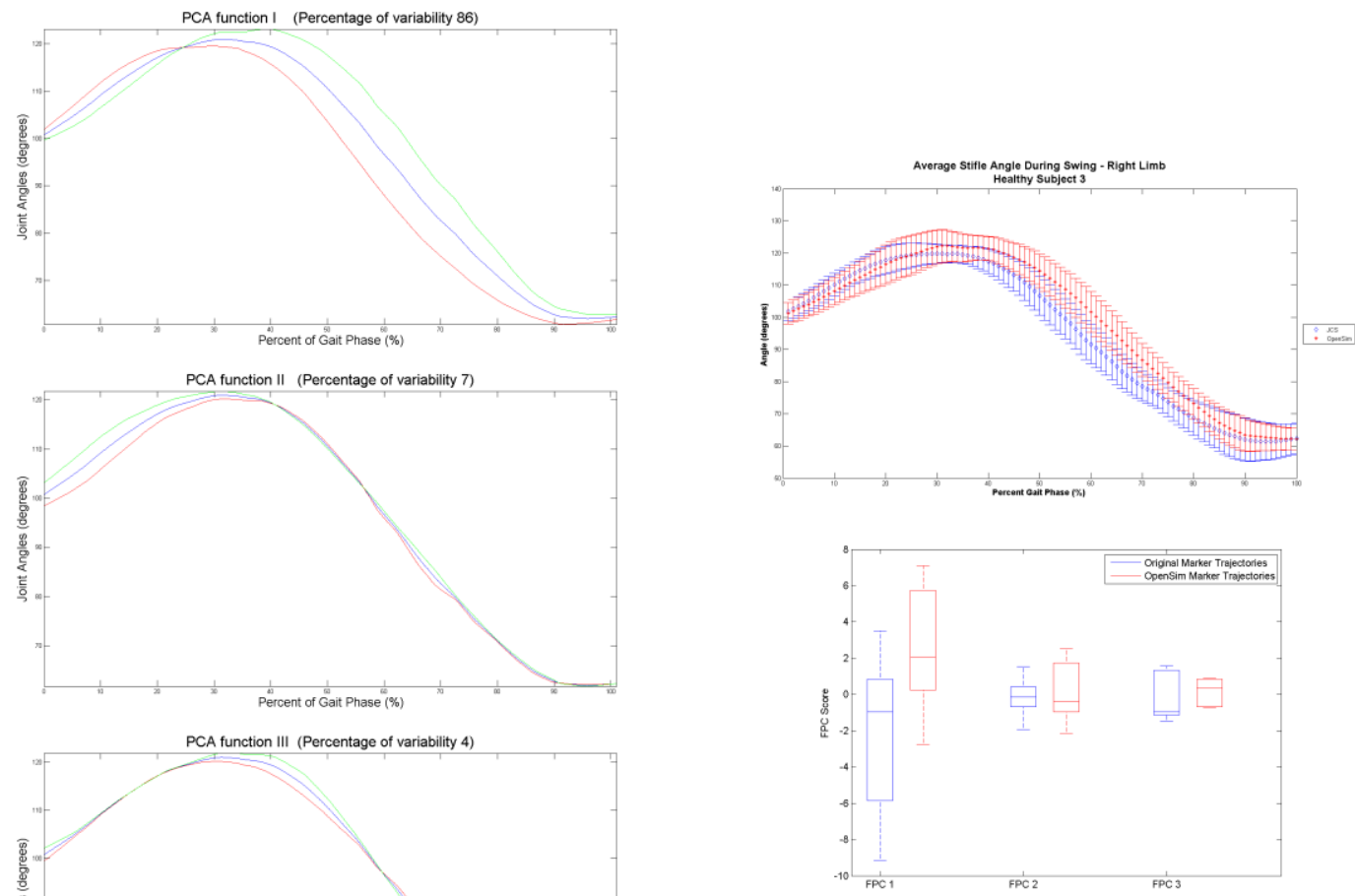

Figure A8. Functional principal component analysis of the second healthy subject for the swing phase of the right stifle joint. A-C) Show the mean curve of the OS and JCS curves in blue, plus (green) or minus (red) the first-third principal components, respectively. D) Mean curves of the JCS curves (blue) compared to the OS curves (red) E) Boxplot of the FPC scores for the first three principal components. 

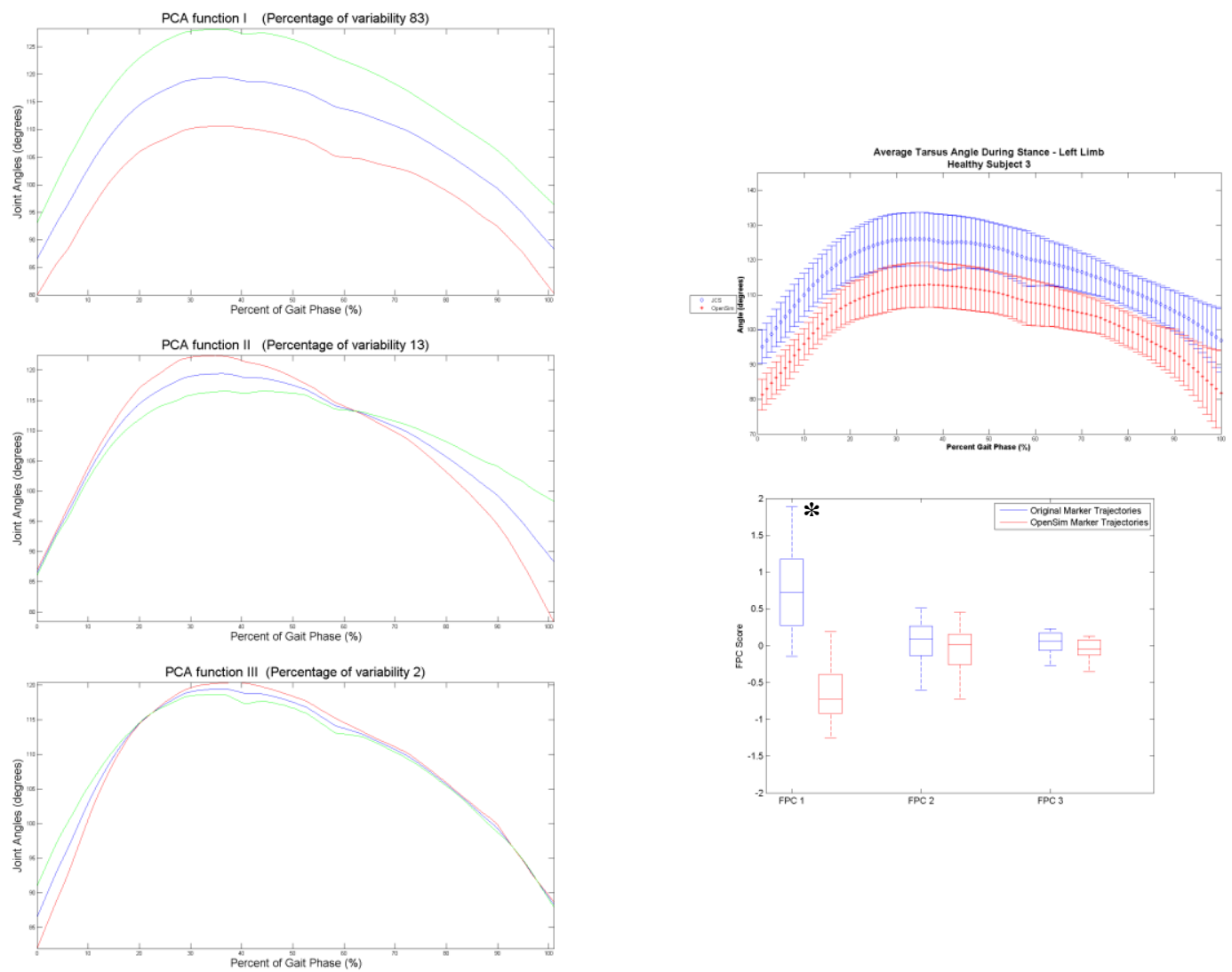

Figure A9. Functional principal component analysis of the second healthy subject for the stance phase of the left tarsus joint. A-C) Show the mean curve of the OS and JCS curves in blue, plus (green) or minus (red) the first-third principal components, respectively. D) Mean curves of the JCS curves (blue) compared to the OS curves (red) E) Boxplot of the FPC scores for the first three principal components. Significant differences in principal component scores are seen in FPC $1(p<0.001)$. 

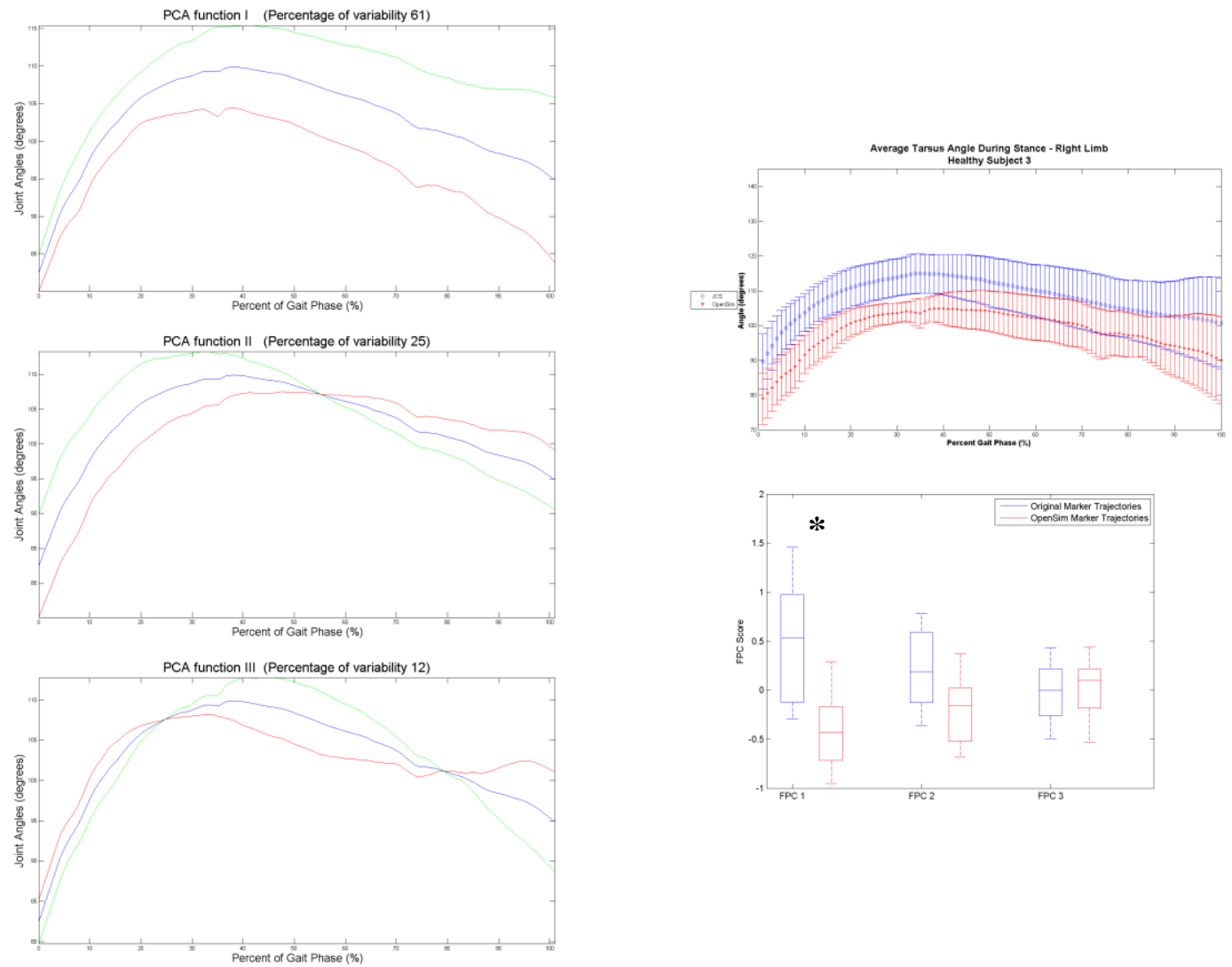

Figure A10. Functional principal component analysis of the second healthy subject for the stance phase of the right tarsus joint. A-C) Show the mean curve of the OS and JCS curves in blue, plus (green) or minus (red) the first-third principal components, respectively. D) Mean curves of the JCS curves (blue) compared to the OS curves (red) E) Boxplot of the FPC scores for the first three principal components. Significant differences in principal component scores are seen in FPC $1(p=0.005)$. 

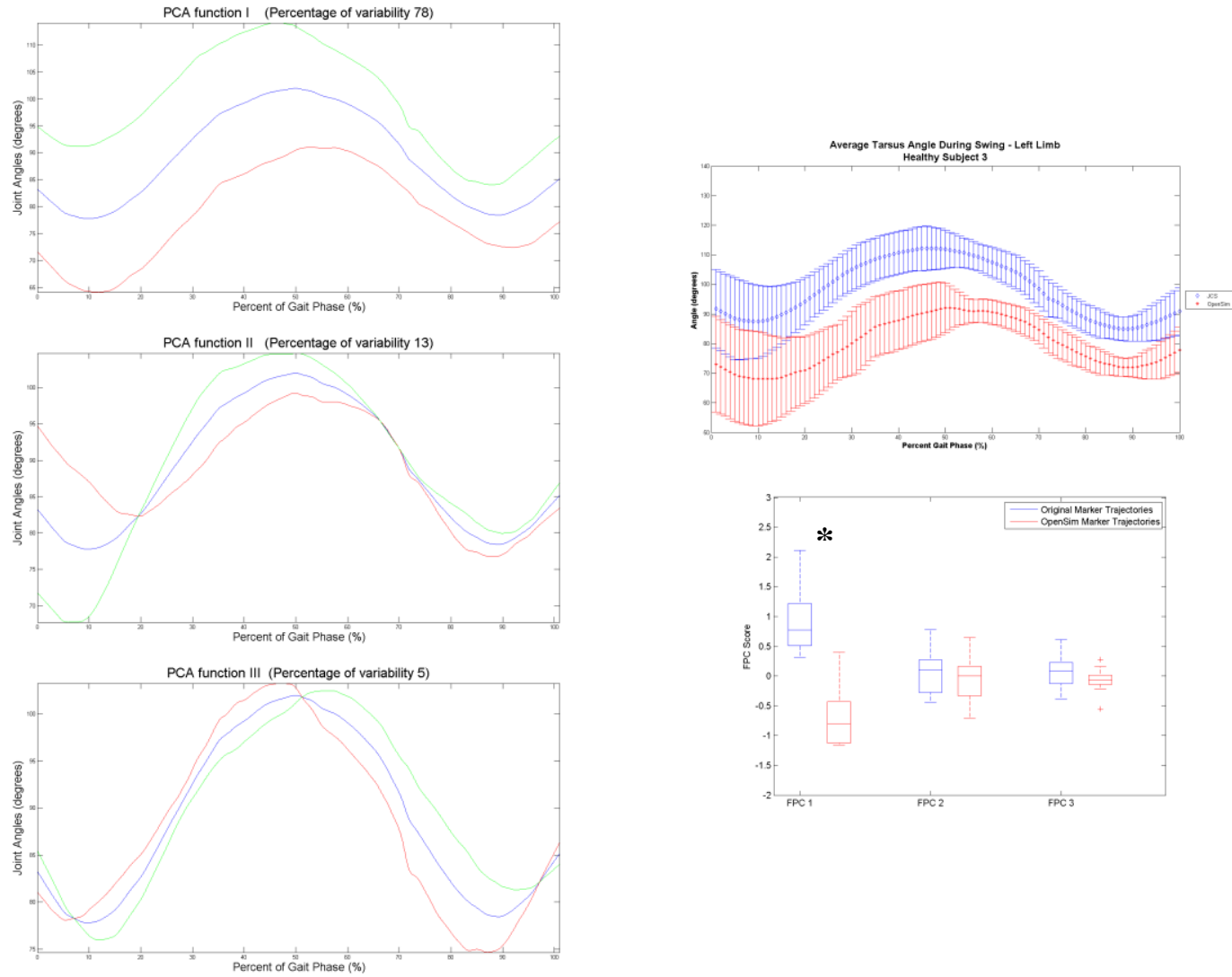

Figure A11. Functional principal component analysis of the second healthy subject for the swing phase of the left tarsus joint. A-C) Show the mean curve of the OS and JCS curves in blue, plus (green) or minus (red) the first-third principal components, respectively. D) Mean curves of the JCS curves (blue) compared to the OS curves (red) E) Boxplot of the FPC scores for the first three principal components. Significant differences in principal component scores are seen in FPC $1(p<0.001)$. 

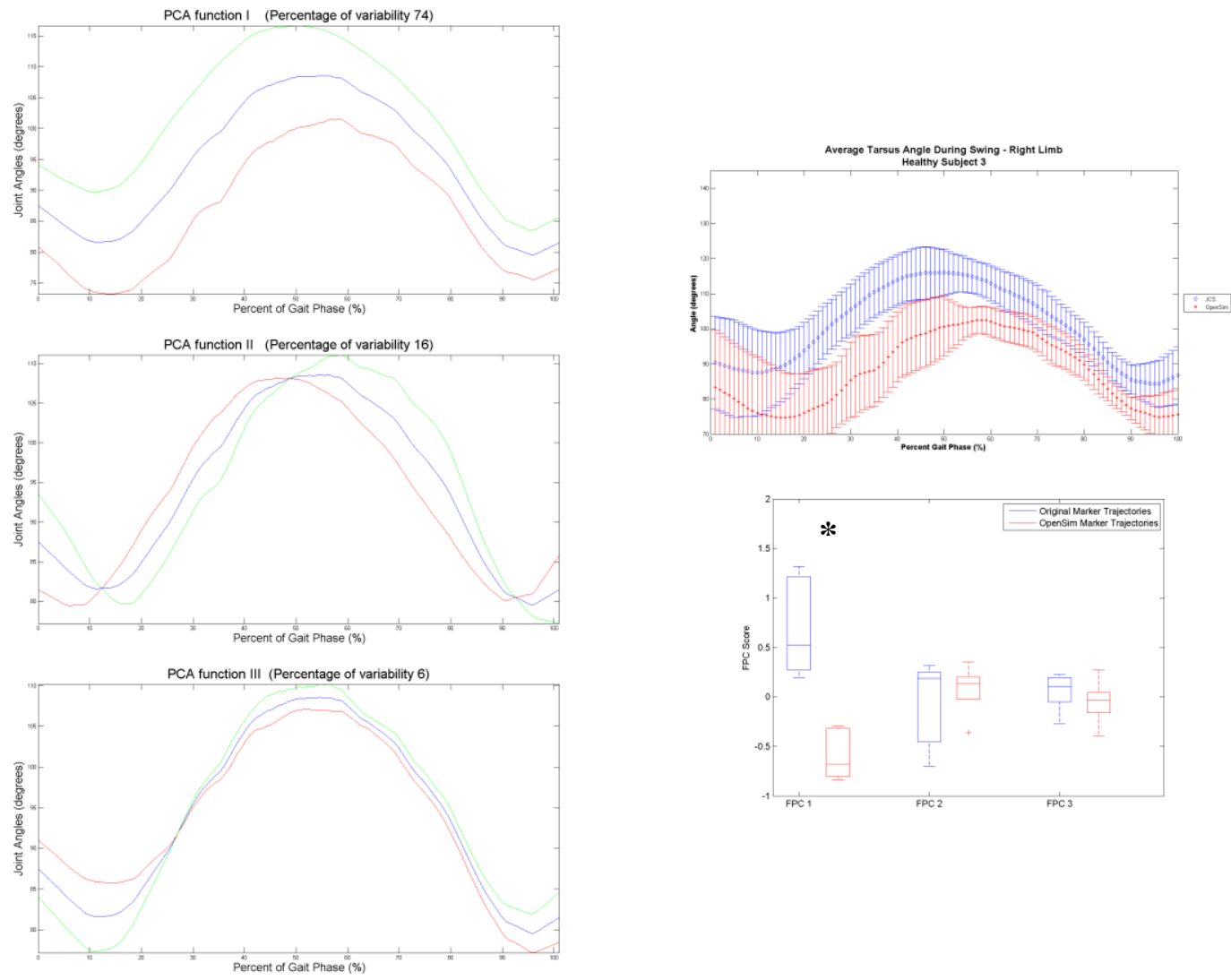

Figure A12. Functional principal component analysis of the second healthy subject for the stance phase of the left tarsus joint. A-C) Show the mean curve of the OS and JCS curves in blue, plus (green) or minus (red) the first-third principal components, respectively. D) Mean curves of the JCS curves (blue) compared to the OS curves (red) E) Boxplot of the FPC scores for the first three principal components. Significant differences in principal component scores are seen in FPC $1(p=0.001)$. 
Appendix B: Functional Principal Components for Healthy Subject 3

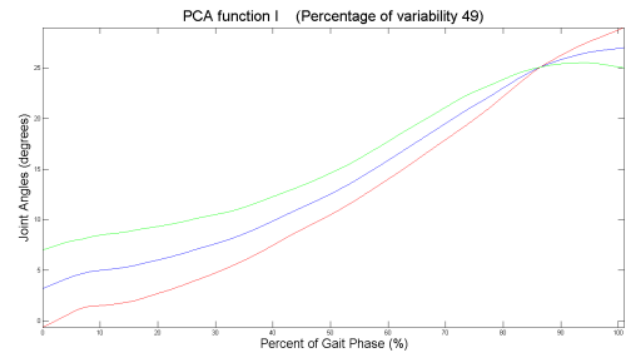

PCA function II (Percentage of variability 44)

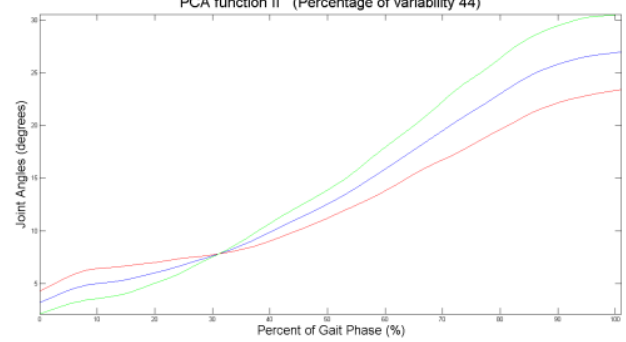

PCA function III (Percentage of variability 4)
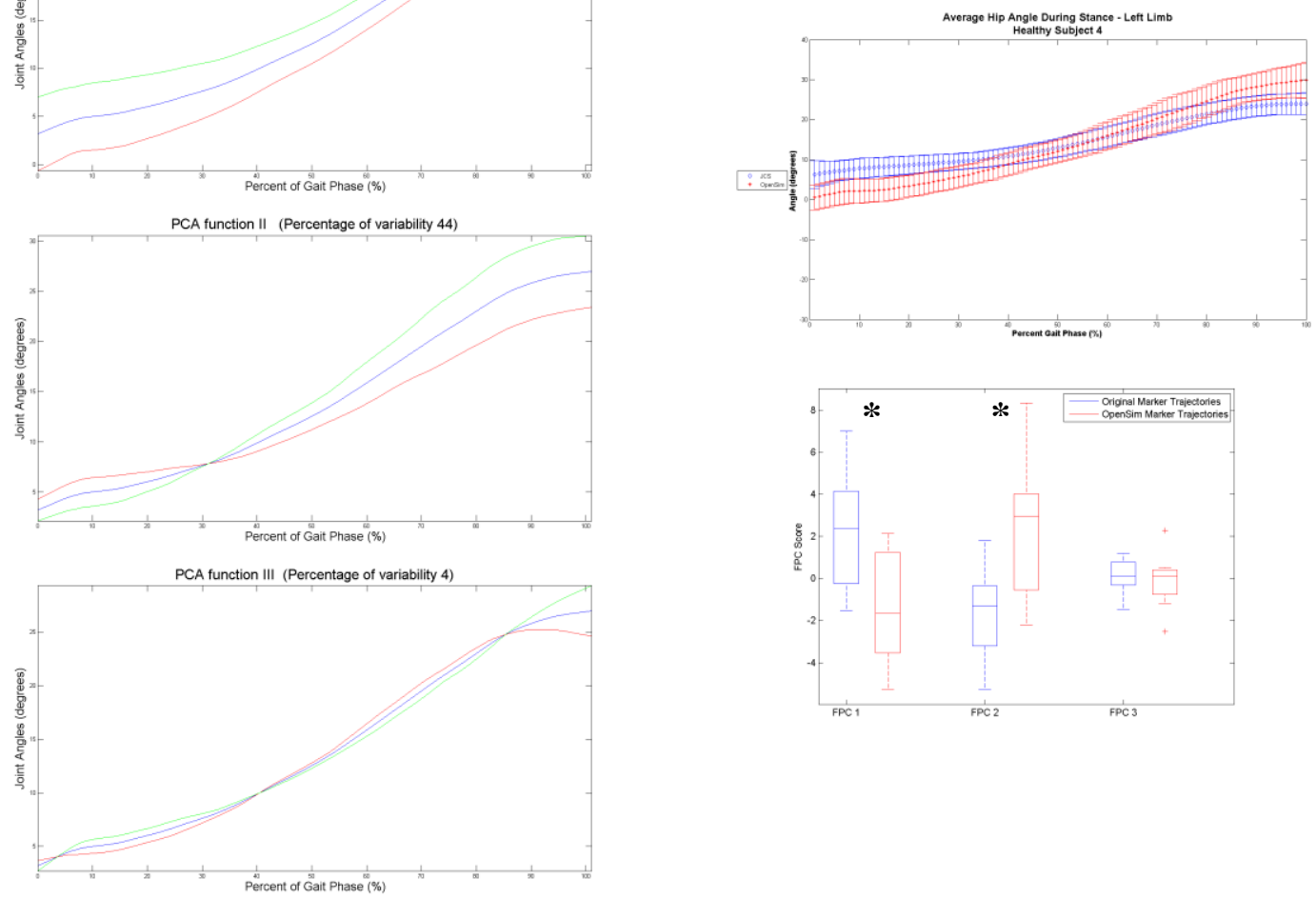

Figure B1. Functional principal component analysis of the third healthy subject for the stance phase of the left hip joint. A-C) Show the mean curve of the OS and JCS curves in blue, plus (green) or minus (red) the first-third principal components, respectively. D) Mean curves of the JCS curves (blue) compared to the OS curves (red) E) Boxplot of the FPC scores for the first three principal components. Significant differences in principal component scores are seen in FPC 1 and 2 ( $p=0.004$ and 0.003, respectively). 

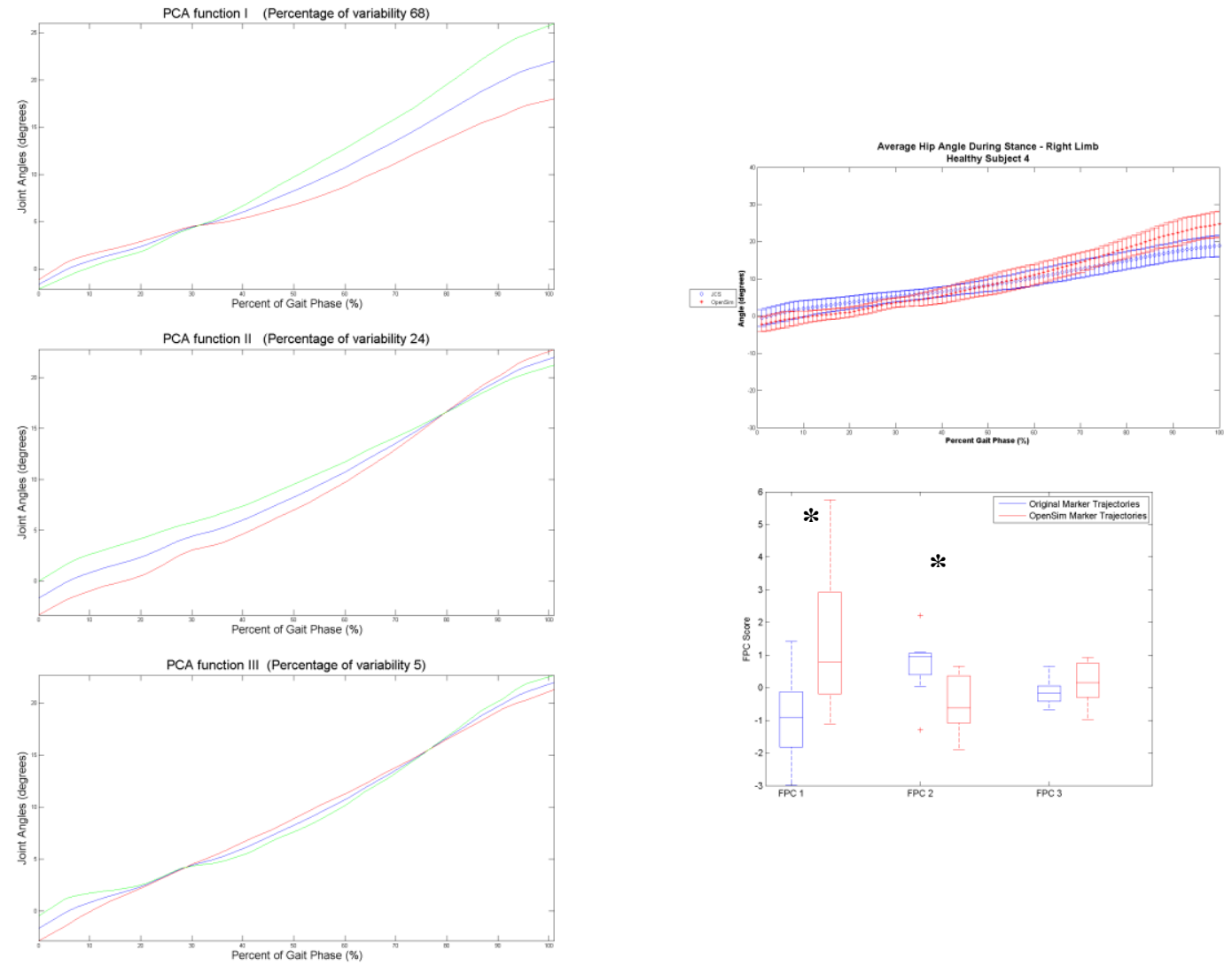

Figure B2. Functional principal component analysis of the third healthy subject for the stance phase of the right hip joint. A-C) Show the mean curve of the OS and JCS curves in blue, plus (green) or minus (red) the first-third principal components, respectively. D) Mean curves of the JCS curves (blue) compared to the OS curves (red) E) Boxplot of the FPC scores for the first three principal components. Significant differences in principal component scores are seen in FPC 1 and 2 ( $p=0.027$ and 0.025, respectively). 

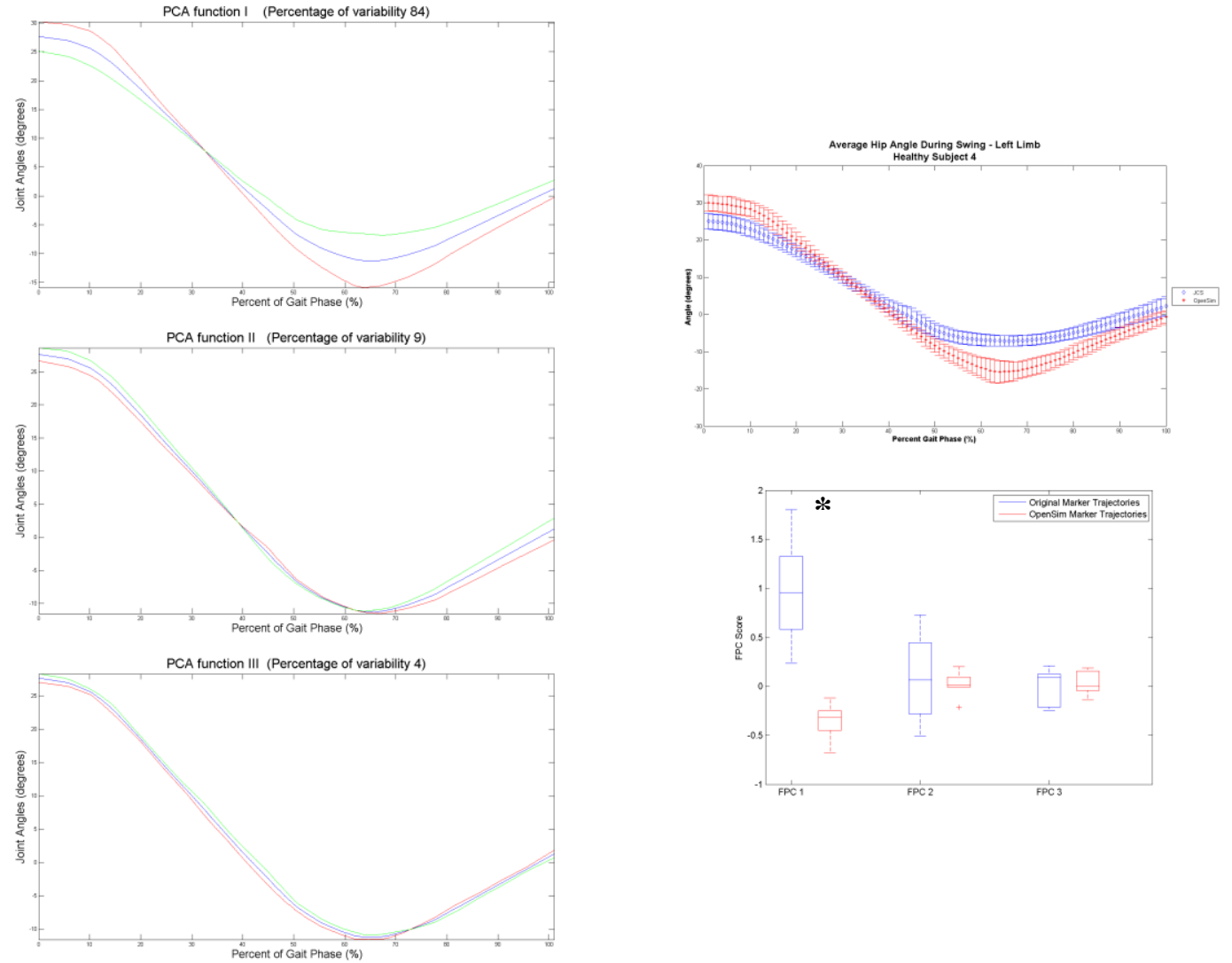

Figure B3. Functional principal component analysis of the third healthy subject for the swing phase of the left hip joint. A-C) Show the mean curve of the OS and JCS curves in blue, plus (green) or minus (red) the first-third principal components, respectively. D) Mean curves of the JCS curves (blue) compared to the OS curves (red) E) Boxplot of the FPC scores for the first three principal components. Significant differences in principal component scores are seen in FPC $1(p<0.001)$. 

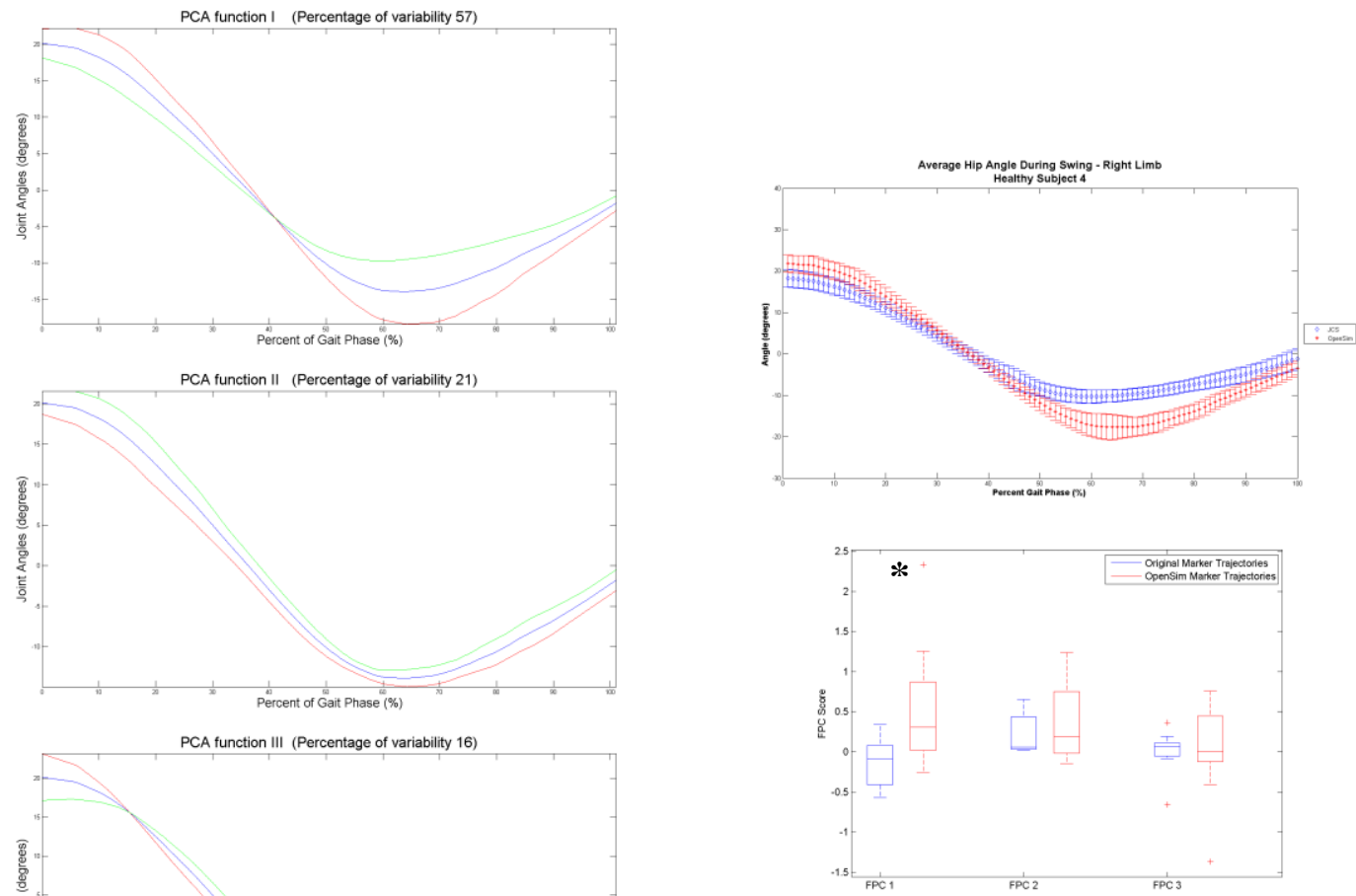

Figure B4. Functional principal component analysis of the third healthy subject for the swing phase of the right hip joint. A-C) Show the mean curve of the OS and JCS curves in blue, plus (green) or minus (red) the first-third principal components, respectively. D) Mean curves of the JCS curves (blue) compared to the OS curves (red) E) Boxplot of the FPC scores for the first three principal components. Significant differences in principal component scores are seen in FPC $1(p=0.009)$. 

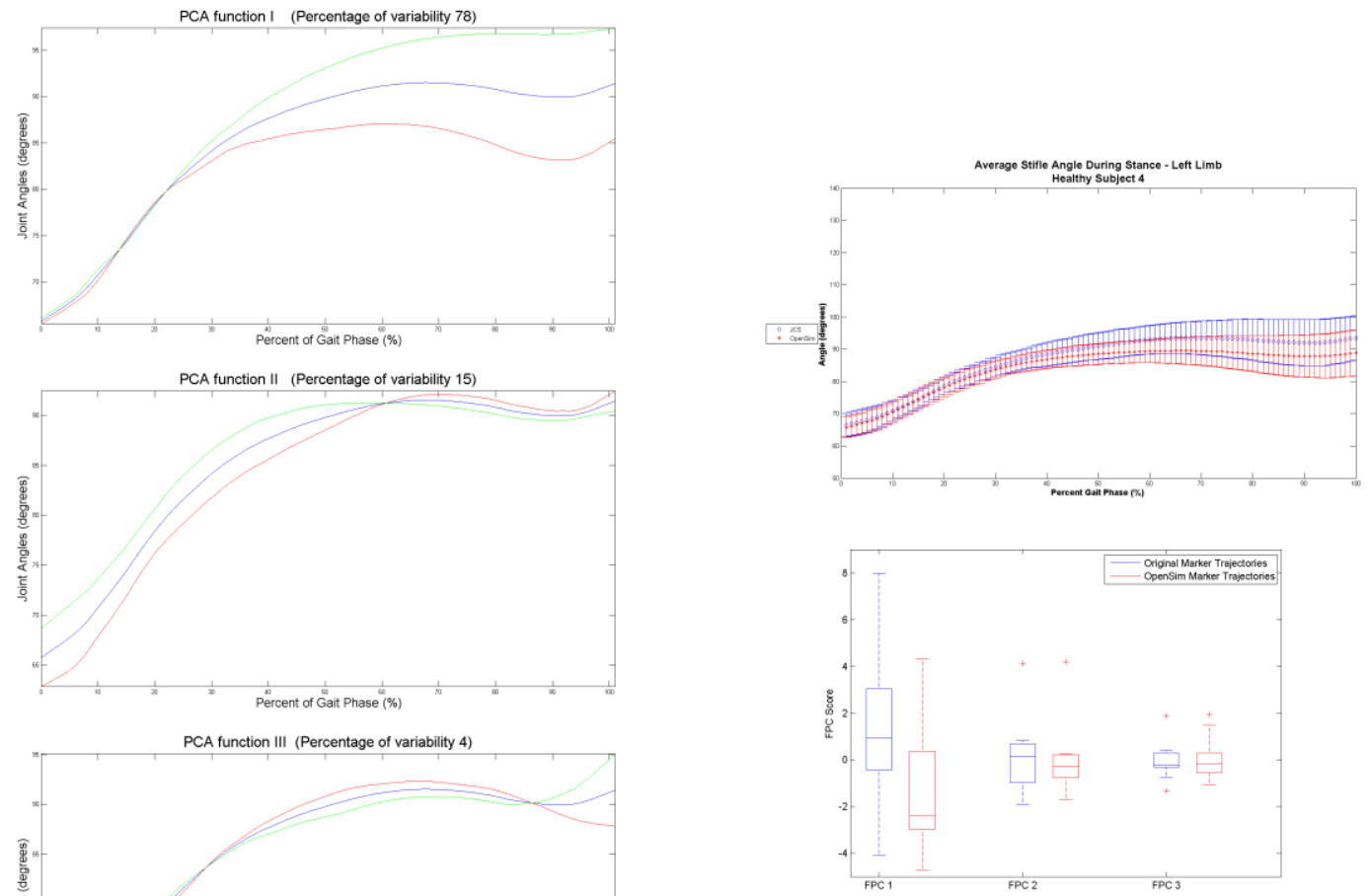

Figure B5. Functional principal component analysis of the third healthy subject for the stance phase of the left stifle joint. A-C) Show the mean curve of the OS and JCS curves in blue, plus (green) or minus (red) the first-third principal components, respectively. D) Mean curves of the JCS curves (blue) compared to the OS curves (red) E) Boxplot of the FPC scores for the first three principal components. 

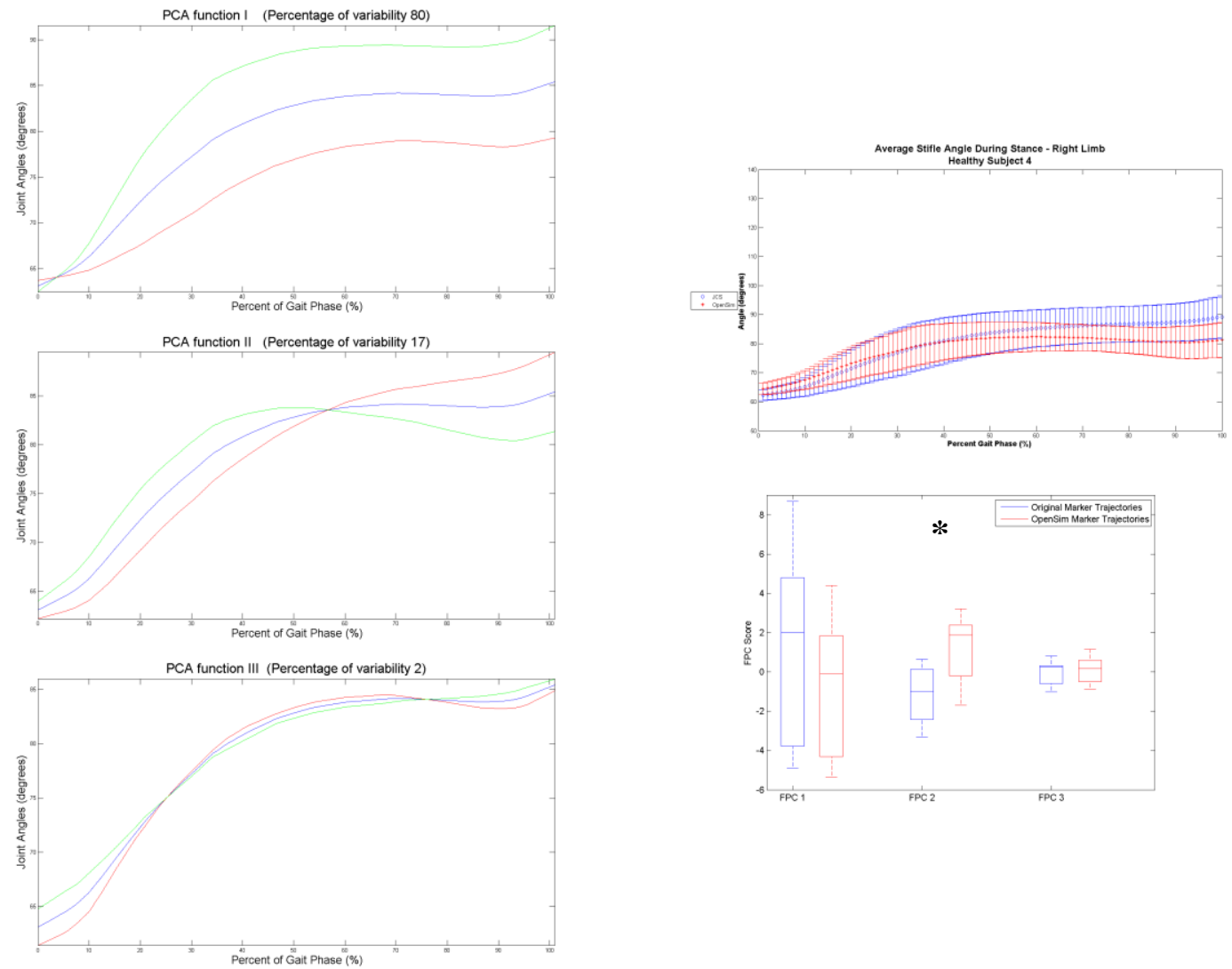

Figure B6. Functional principal component analysis of the third healthy subject for the stance phase of the right stifle joint. A-C) Show the mean curve of the OS and JCS curves in blue, plus (green) or minus (red) the first-third principal components, respectively. D) Mean curves of the JCS curves (blue) compared to the OS curves (red) E) Boxplot of the FPC scores for the first three principal components. Significant differences in principal component scores are seen in FPC $2(p=0.005)$. 

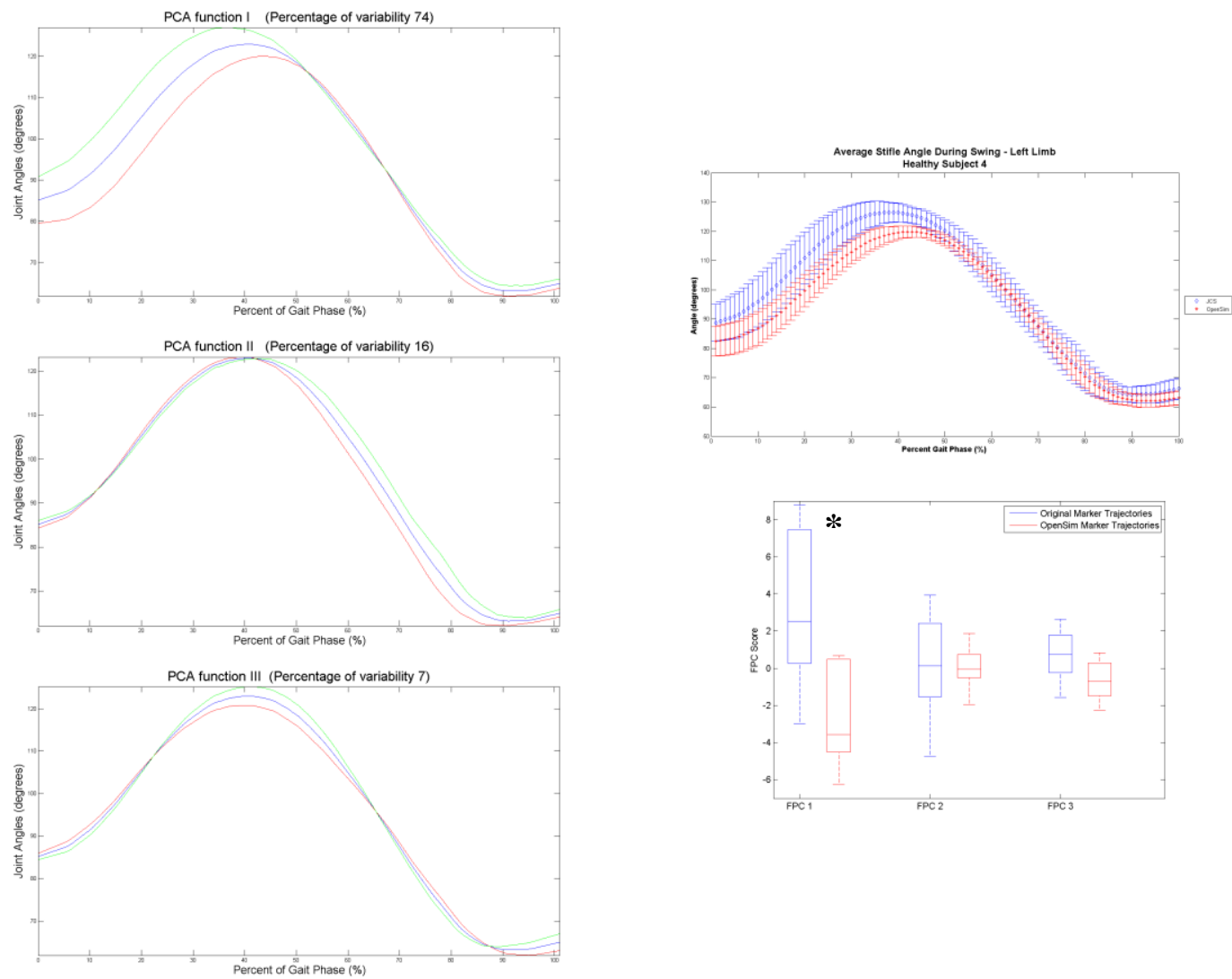

Figure B7. Functional principal component analysis of the third healthy subject for the swing phase of the left stifle joint. A-C) Show the mean curve of the OS and JCS curves in blue, plus (green) or minus (red) the first-third principal components, respectively. D) Mean curves of the JCS curves (blue) compared to the OS curves (red) E) Boxplot of the FPC scores for the first three principal components. Significant differences in principal component scores are seen in FPC $1(p=0.023)$. 

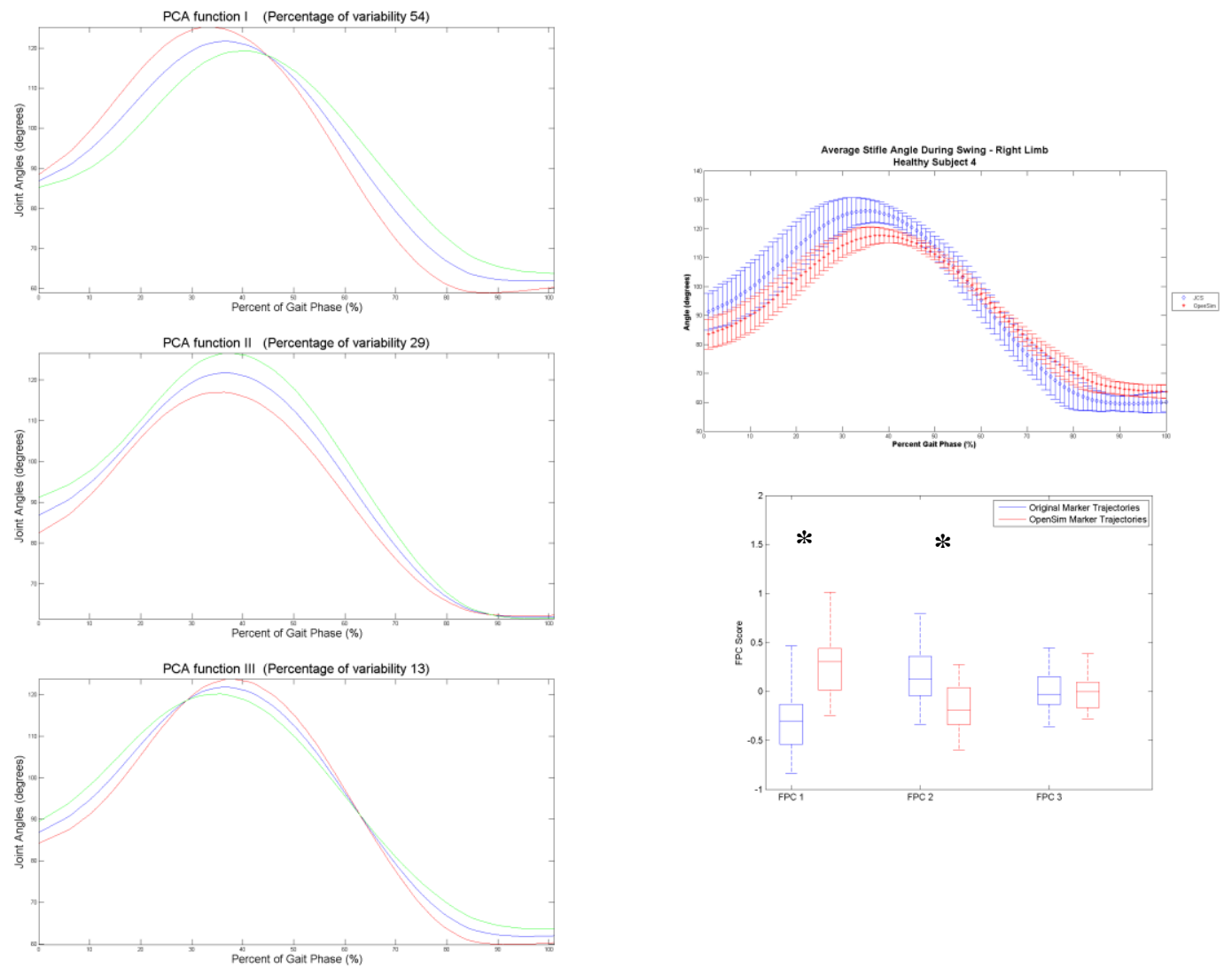

Figure B8. Functional principal component analysis of the third healthy subject for the swing phase of the right stifle joint. A-C) Show the mean curve of the OS and JCS curves in blue, plus (green) or minus (red) the first-third principal components, respectively. D) Mean curves of the JCS curves (blue) compared to the OS curves (red) E) Boxplot of the FPC scores for the first three principal components. Significant differences in principal component scores are seen in FPC 1 and $2(p<0.001$ and $p=0.013$, respectively). 

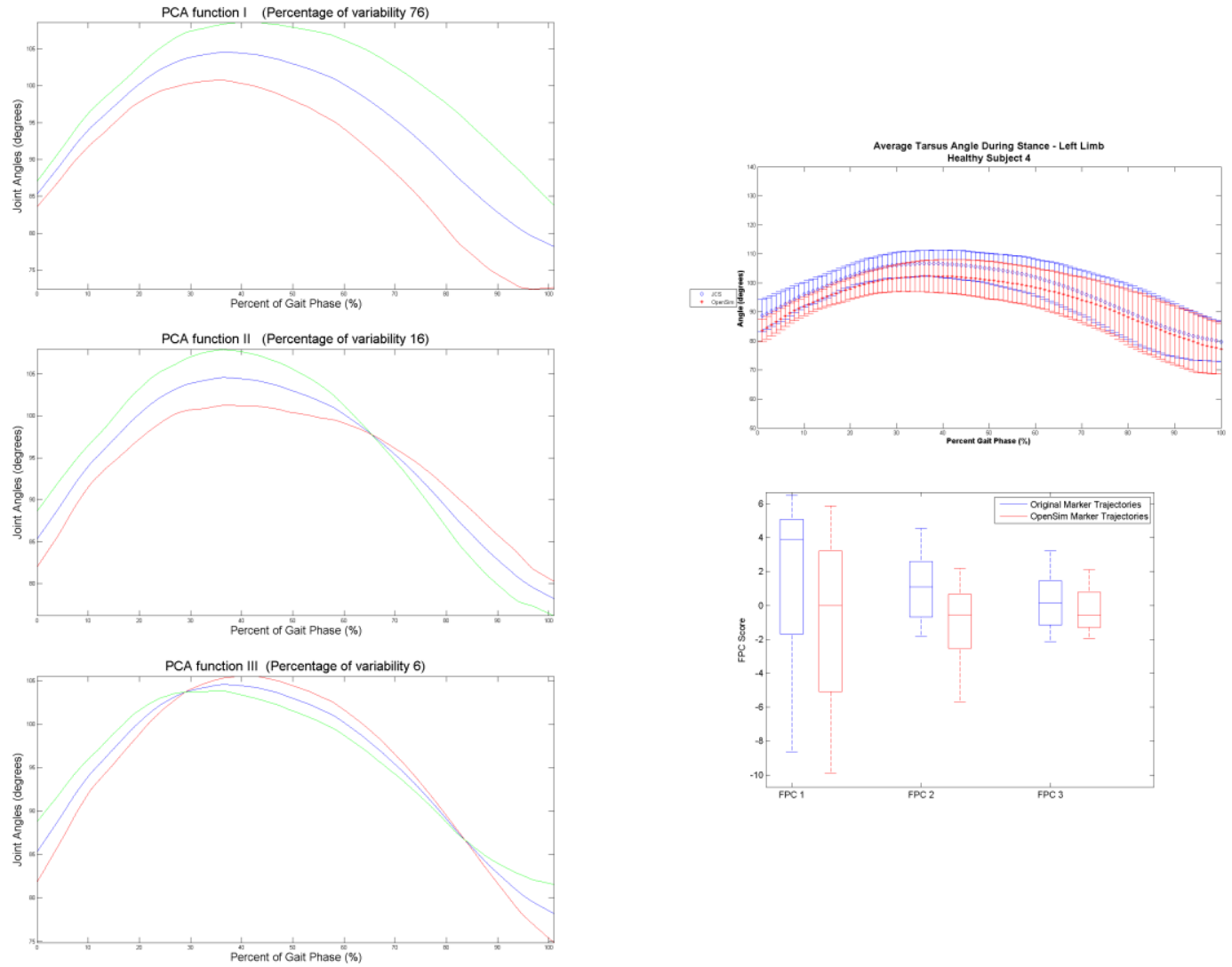

Figure B9. Functional principal component analysis of the third healthy subject for the stance phase of the left tarsus joint. A-C) Show the mean curve of the OS and JCS curves in blue, plus (green) or minus (red) the first-third principal components, respectively. D) Mean curves of the JCS curves (blue) compared to the OS curves (red) E) Boxplot of the FPC scores for the first three principal components. 

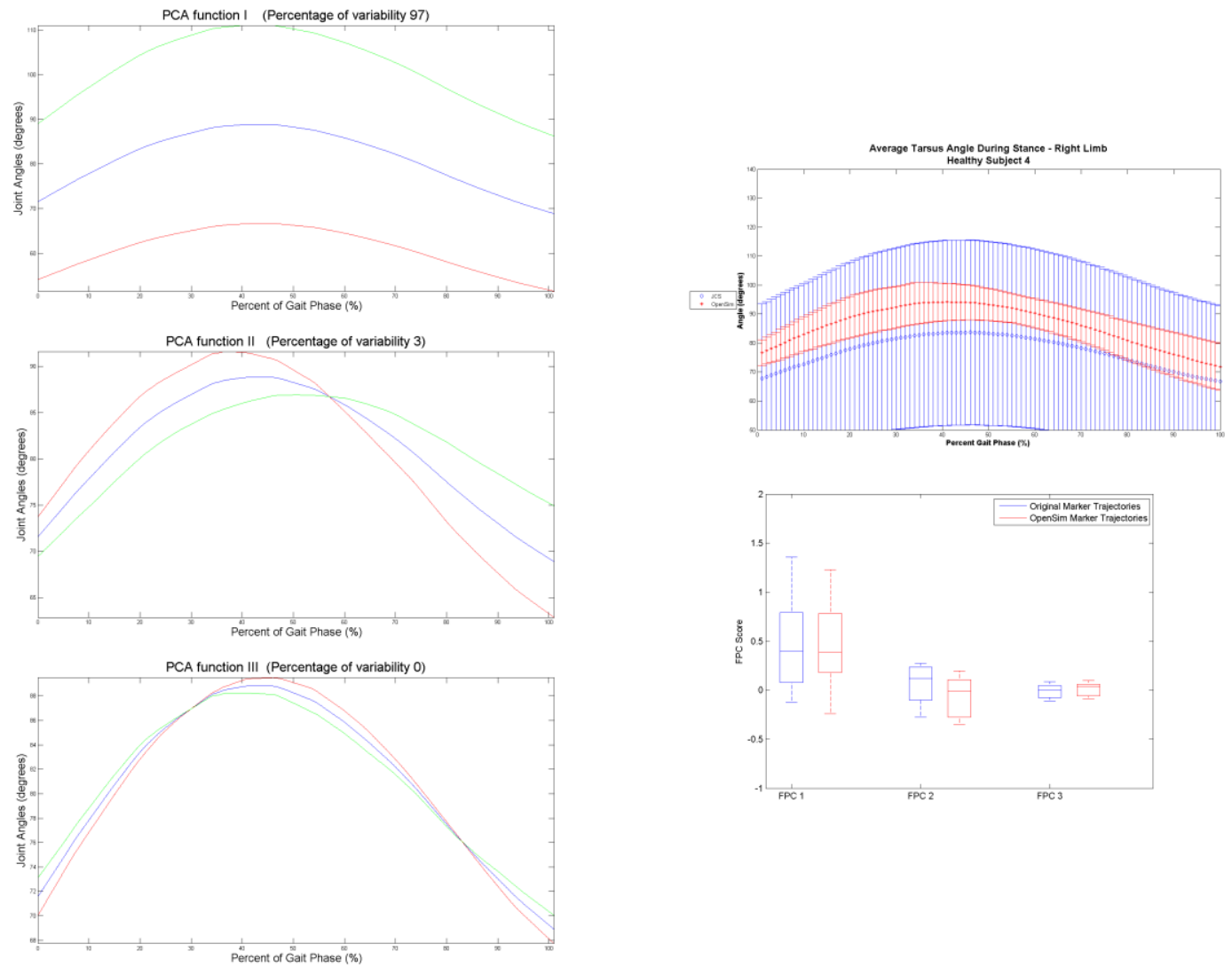

Figure B10. Functional principal component analysis of the third healthy subject for the stance phase of the right tarsus joint. A-C) Show the mean curve of the OS and JCS curves in blue, plus (green) or minus (red) the first-third principal components, respectively. D) Mean curves of the JCS curves (blue) compared to the OS curves (red) E) Boxplot of the FPC scores for the first three principal components. 

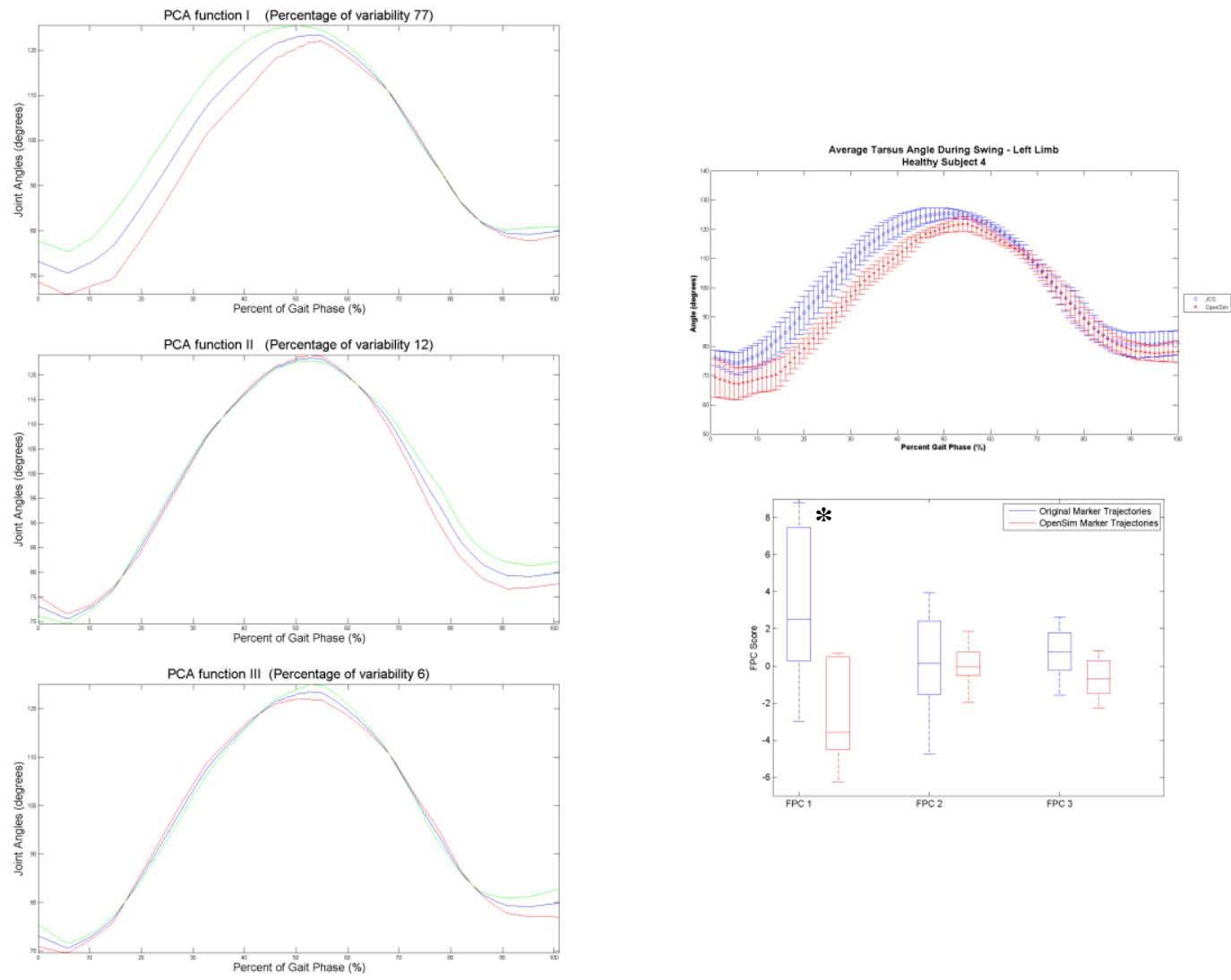

Figure B11. Functional principal component analysis of the third healthy subject for the swing phase of the left tarsus joint. A-C) Show the mean curve of the OS and JCS curves in blue, plus (green) or minus (red) the first-third principal components, respectively. D) Mean curves of the JCS curves (blue) compared to the OS curves (red) E) Boxplot of the FPC scores for the first three principal components. Significant differences in principal component scores are seen in FPC $1(p=0.007)$. 

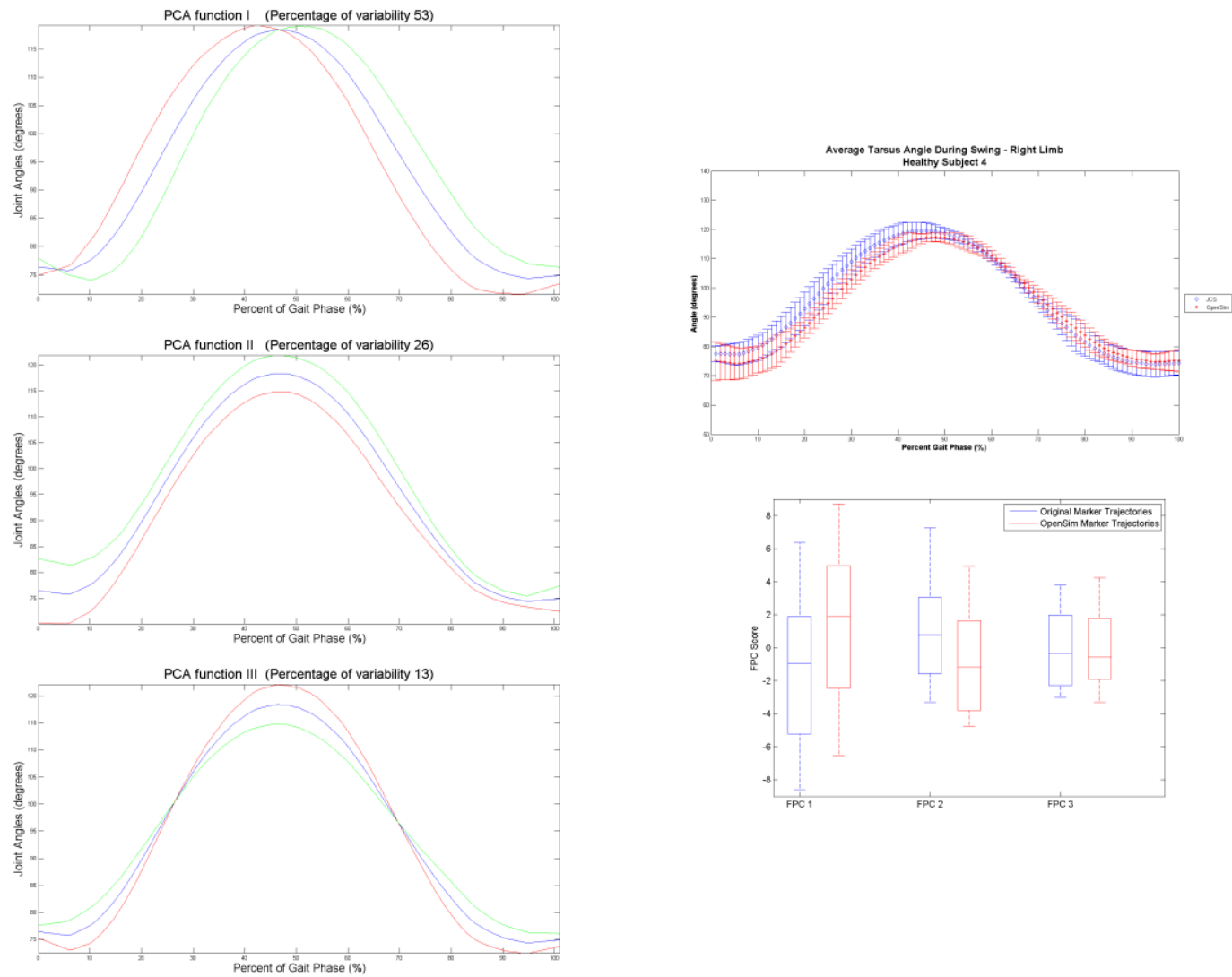

Figure B12. Functional principal component analysis of the third healthy subject for the swing phase of the right tarsus joint. A-C) Show the mean curve of the OS and JCS curves in blue, plus (green) or minus (red) the first-third principal components, respectively. D) Mean curves of the JCS curves (blue) compared to the OS curves (red) E) Boxplot of the FPC scores for the first three principal components. 


\section{Appendix C: Muscle Activations of All Healthy Subjects}
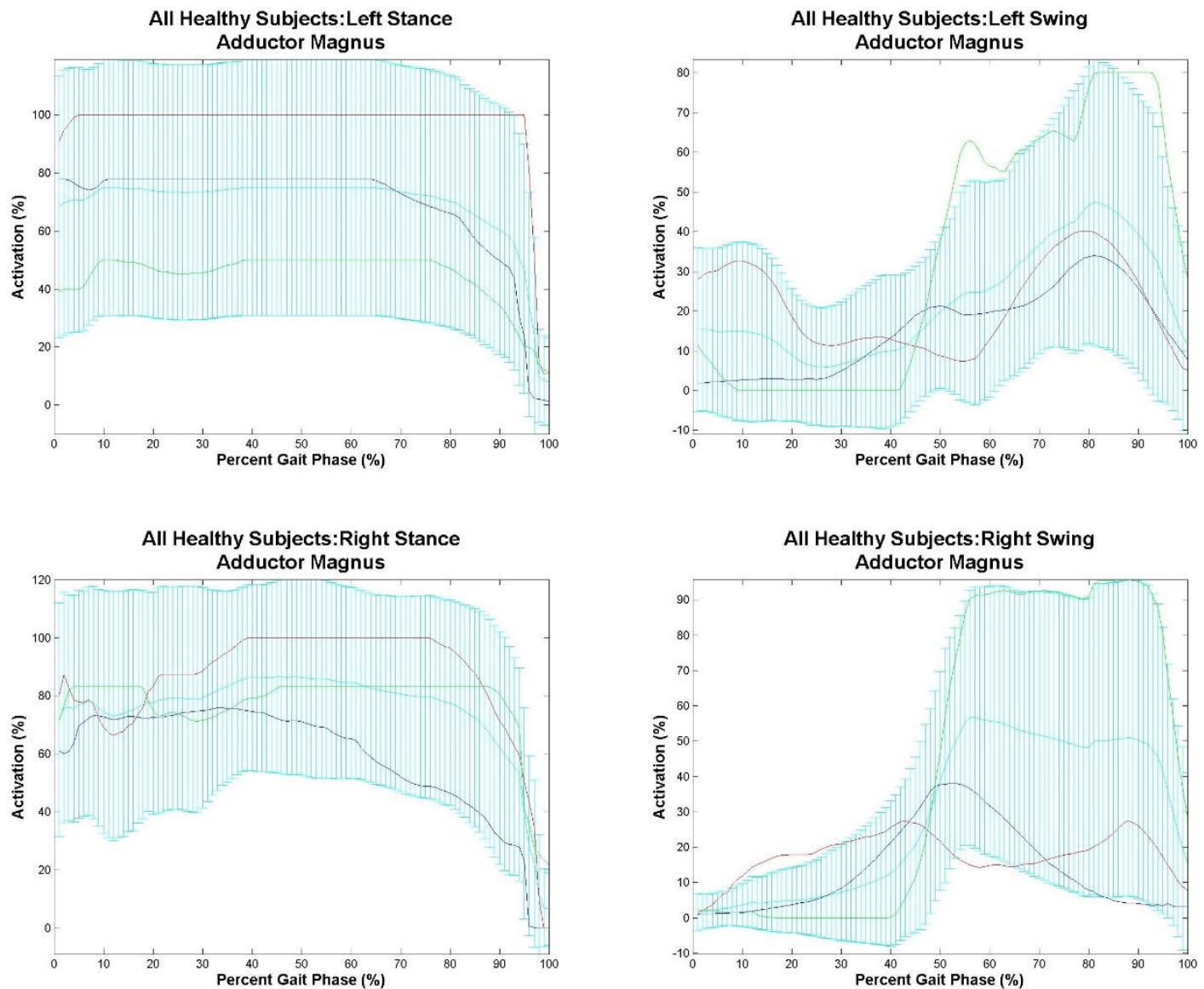

Figure $\mathrm{C} 1$. Average adductor magnus activation of all healthy subjects. Healthy subject 1 is shown in dark blue, healthy subject 2 is shown in red, and healthy subject 3 is shown in green. Average activation of all three subjects is displayed with standard error in cyan. 

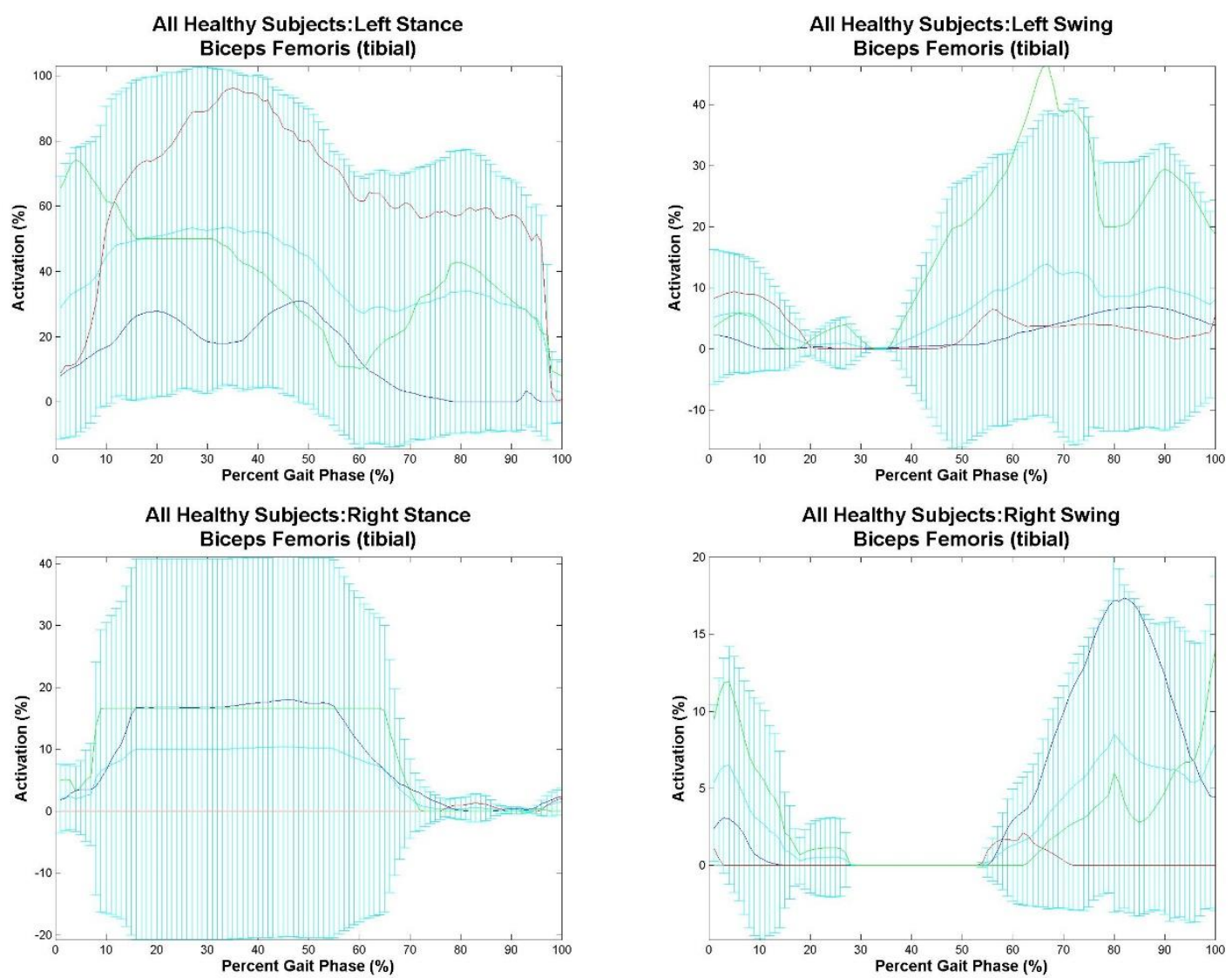

Figure C2. Average biceps femoris activation of all healthy subjects. Healthy subject 1 is shown in dark blue, healthy subject 2 is shown in red, and healthy subject 3 is shown in green. Average activation of all three subjects is displayed with standard error in cyan. 

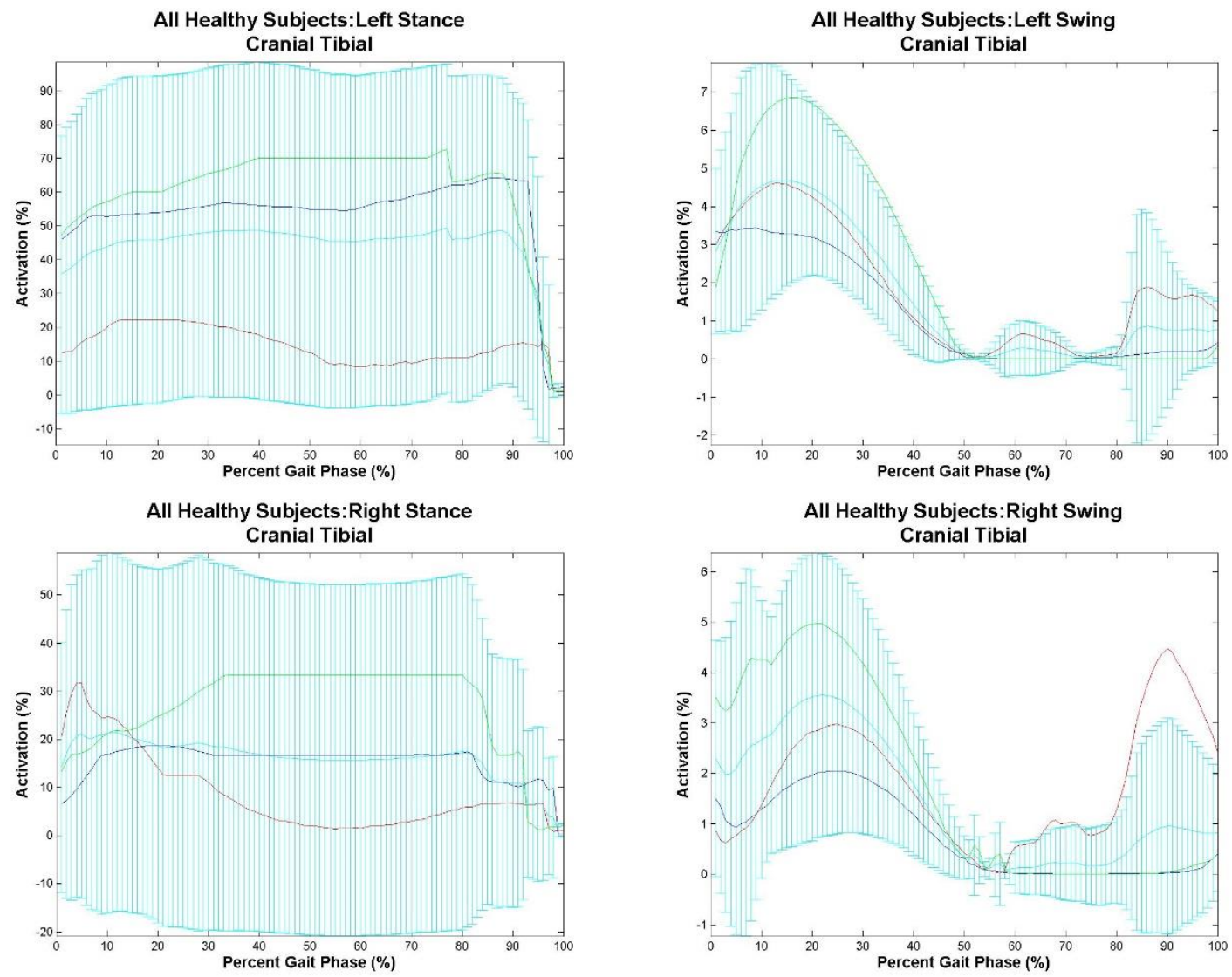

Figure C3. Average cranial tibial activation of all healthy subjects. Healthy subject 1 is shown in dark blue, healthy subject 2 is shown in red, and healthy subject 3 is shown in green. Average activation of all three subjects is displayed with standard error in cyan. 

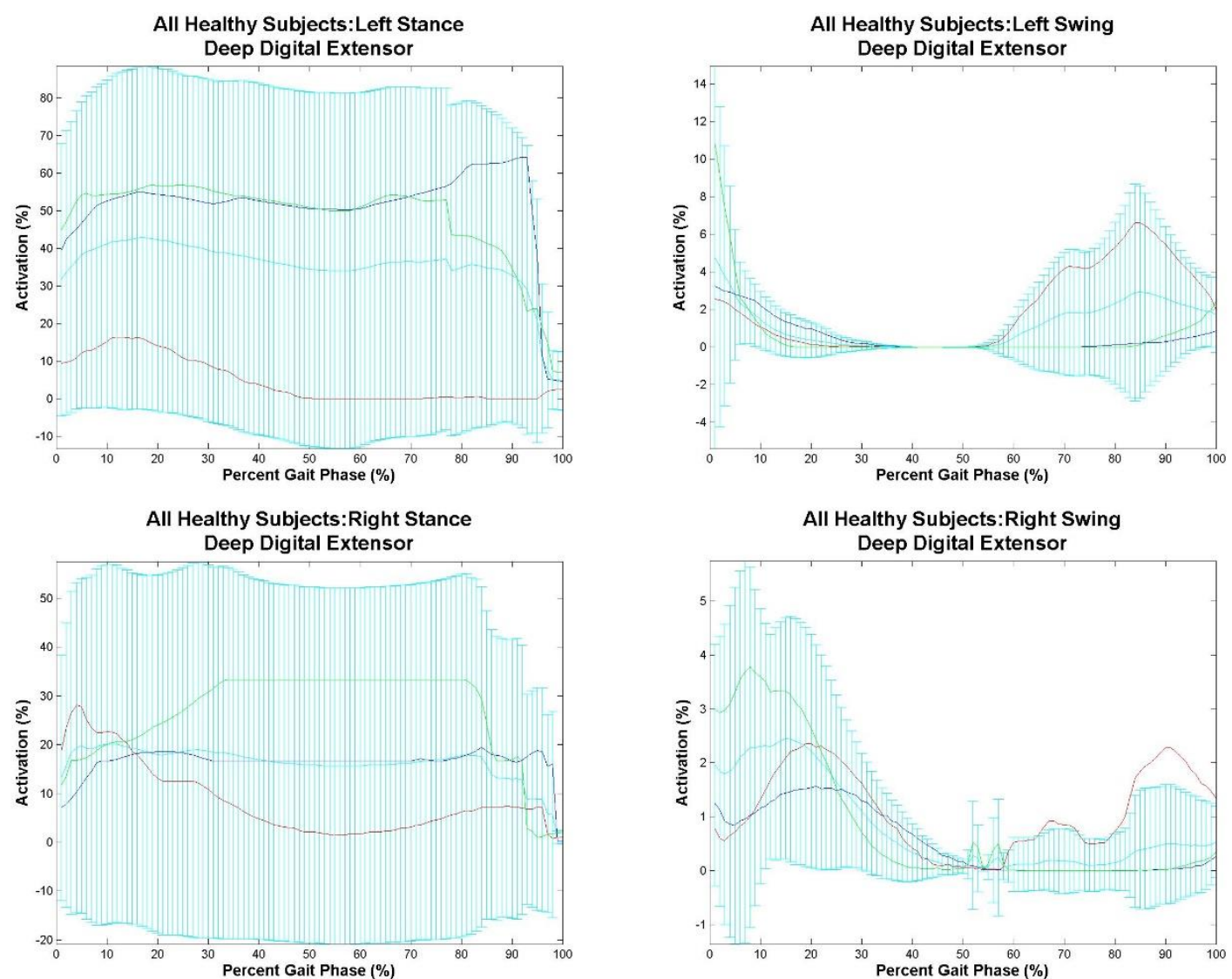

Figure C4. Average long digital extensor activation of all healthy subjects. Healthy subject 1 is shown in dark blue, healthy subject 2 is shown in red, and healthy subject 3 is shown in green. Average activation of all three subjects is displayed with standard error in cyan. 

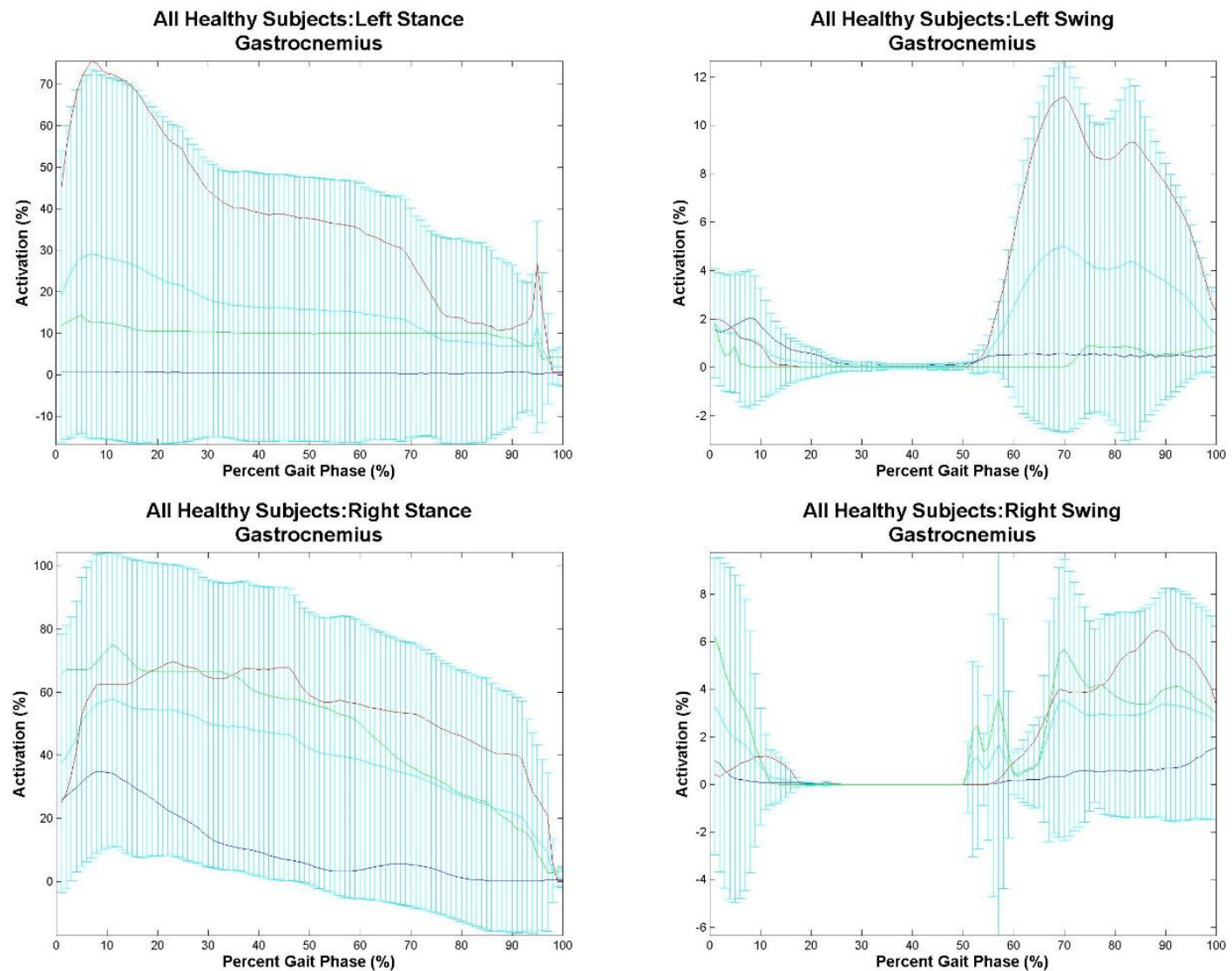

Figure C5. Average gastrocnemius activation of all healthy subjects. Healthy subject 1 is shown in dark blue, healthy subject 2 is shown in red, and healthy subject 3 is shown in green. Average activation of all three subjects is displayed with standard error in cyan. 

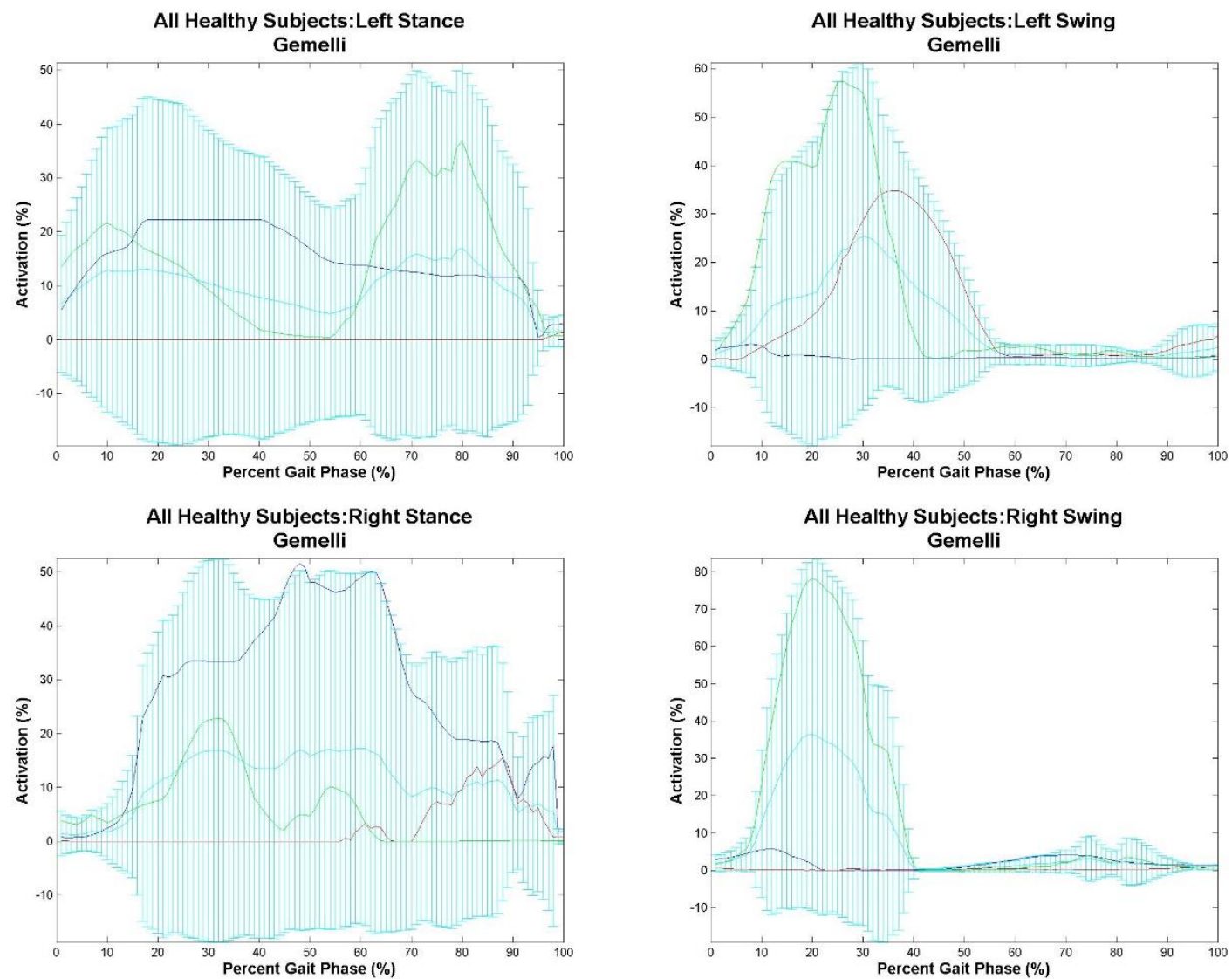

Figure C6. Average gemellus activation of all healthy subjects. Healthy subject 1 is shown in dark blue, healthy subject 2 is shown in red, and healthy subject 3 is shown in green. Average activation of all three subjects is displayed with standard error in cyan. 

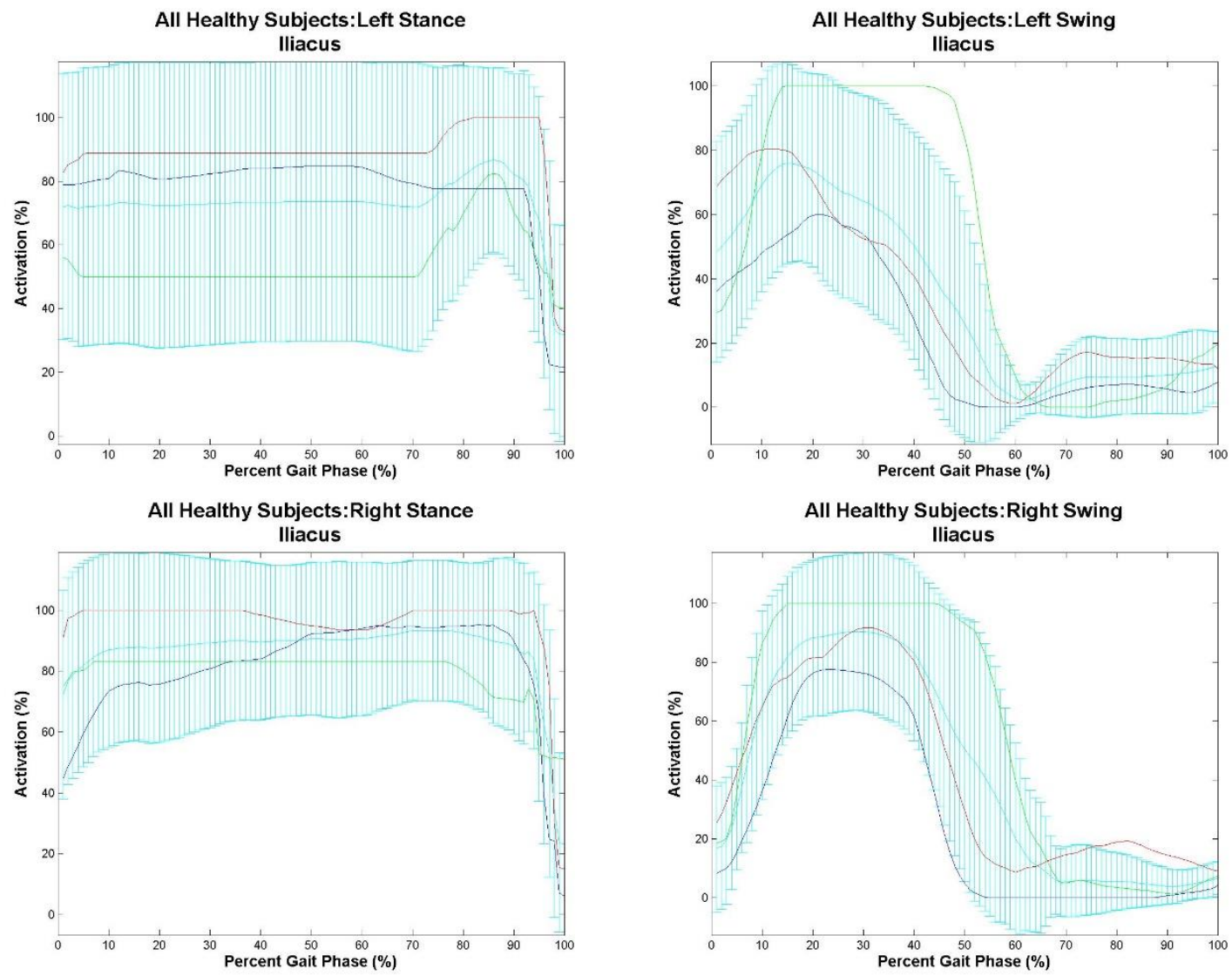

Figure C7. Average iliacus activation of all healthy subjects. Healthy subject 1 is shown in dark blue, healthy subject 2 is shown in red, and healthy subject 3 is shown in green.

Average activation of all three subjects is displayed with standard error in cyan. 

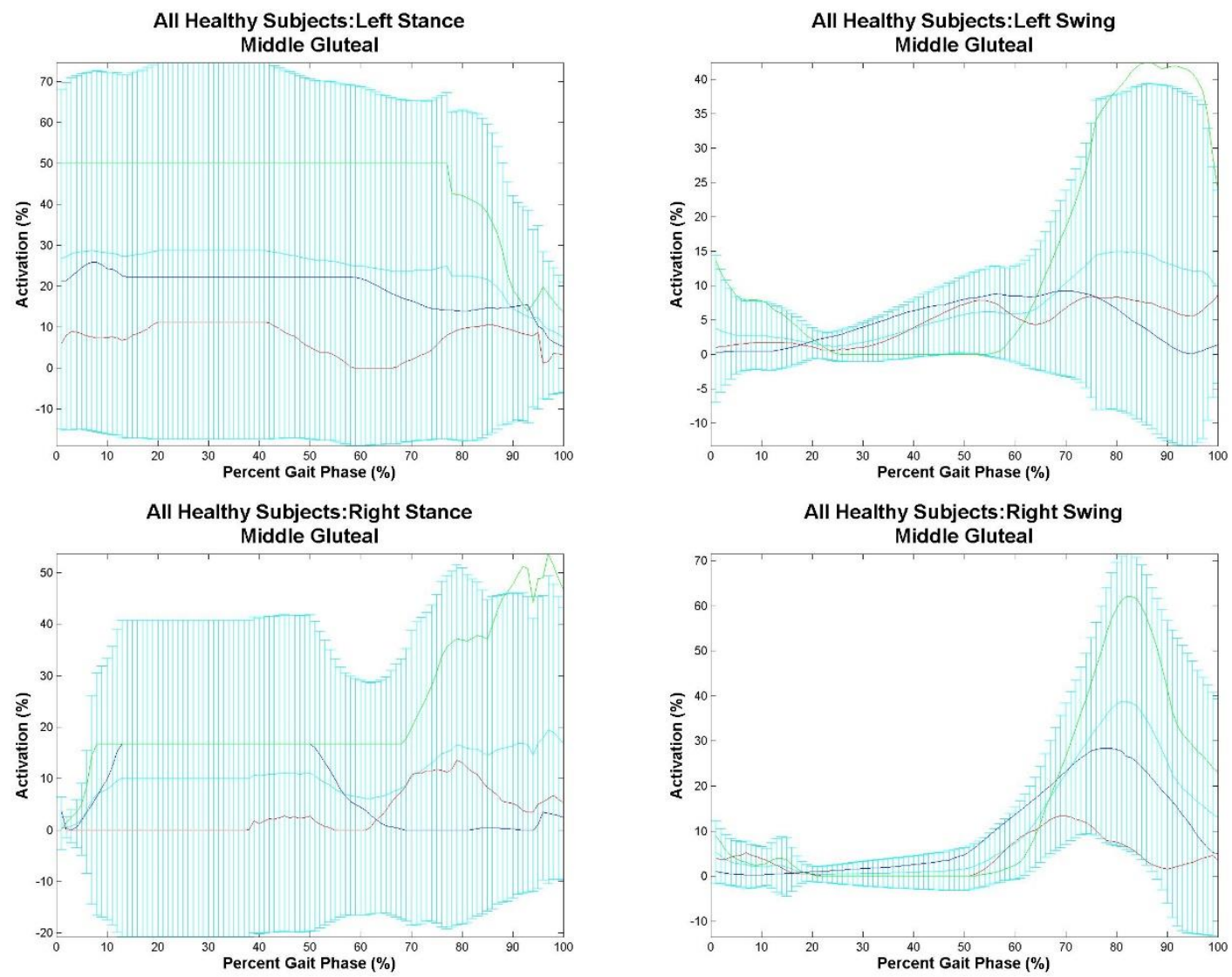

Figure C8. Average middle gluteal activation of all healthy subjects. Healthy subject 1 is shown in dark blue, healthy subject 2 is shown in red, and healthy subject 3 is shown in green. Average activation of all three subjects is displayed with standard error in cyan. 

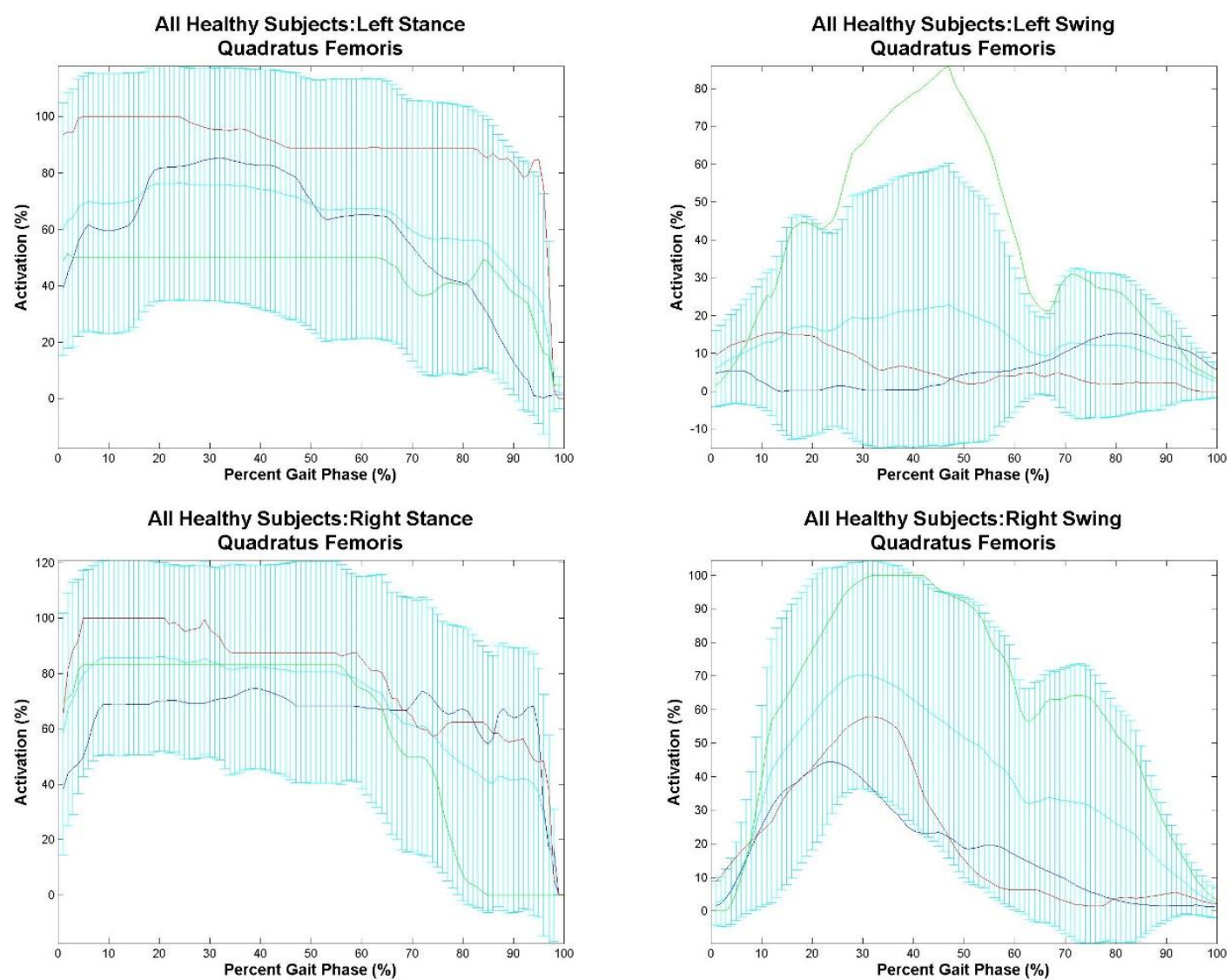

Figure C9. Average quadratus femoris activation of all healthy subjects. Healthy subject 1 is shown in dark blue, healthy subject 2 is shown in red, and healthy subject 3 is shown in green. Average activation of all three subjects is displayed with standard error in cyan. 

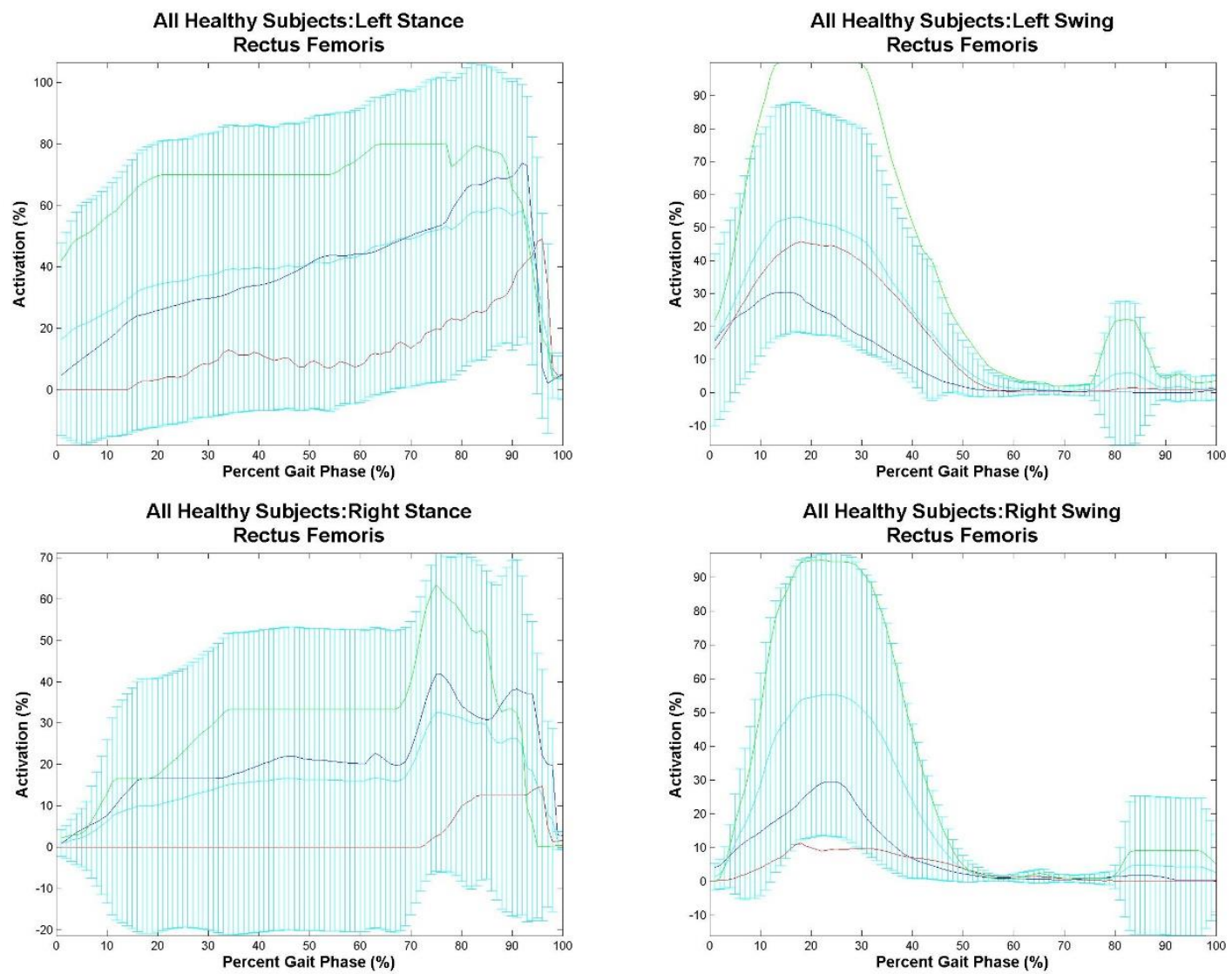

Figure C10. Average rectus femoris activation of all healthy subjects. Healthy subject 1 is shown in dark blue, healthy subject 2 is shown in red, and healthy subject 3 is shown in green. Average activation of all three subjects is displayed with standard error in cyan. 

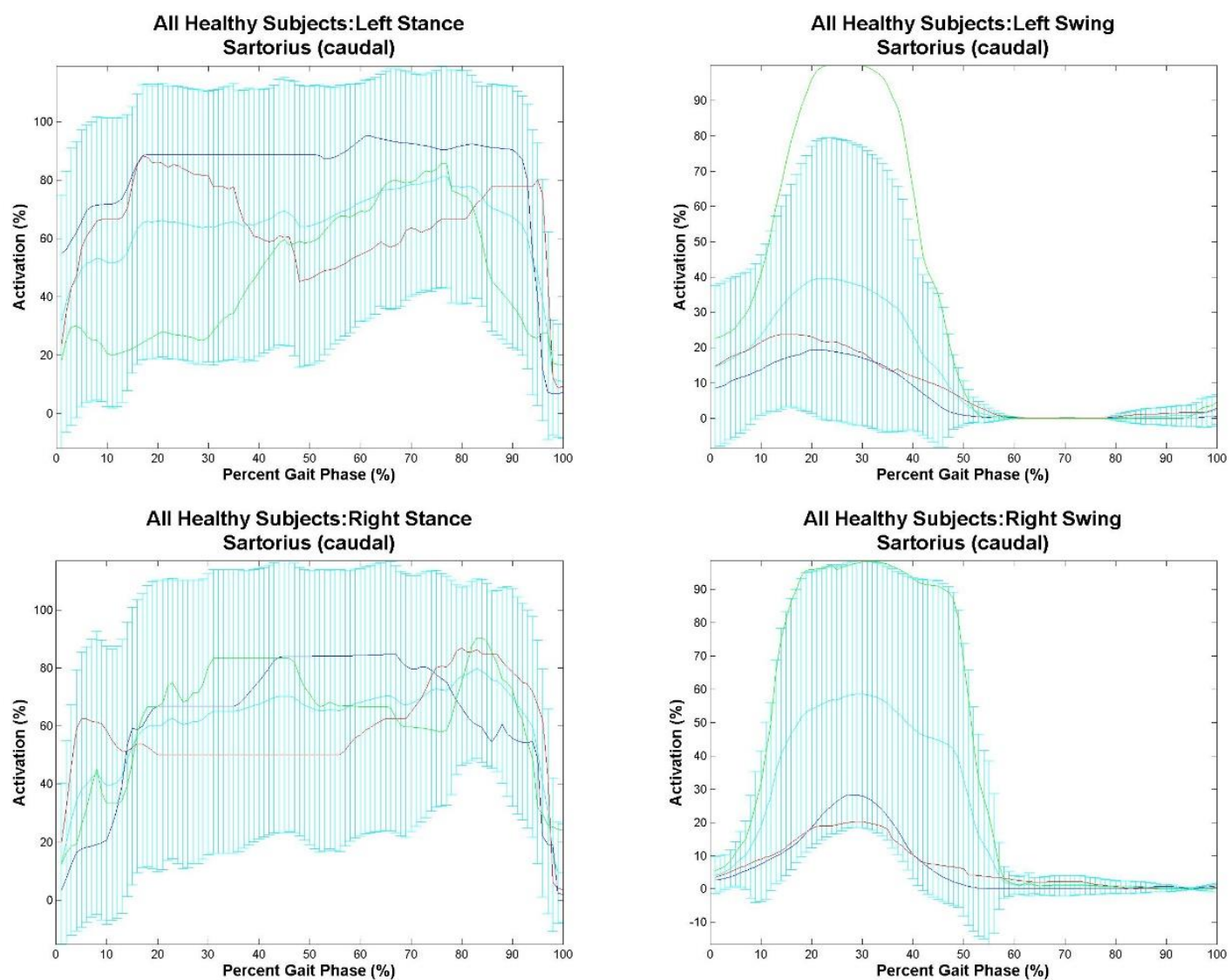

Figure C11. Average caudal Sartorius activation of all healthy subjects. Healthy subject 1 is shown in dark blue, healthy subject 2 is shown in red, and healthy subject 3 is shown in green. Average activation of all three subjects is displayed with standard error in cyan. 

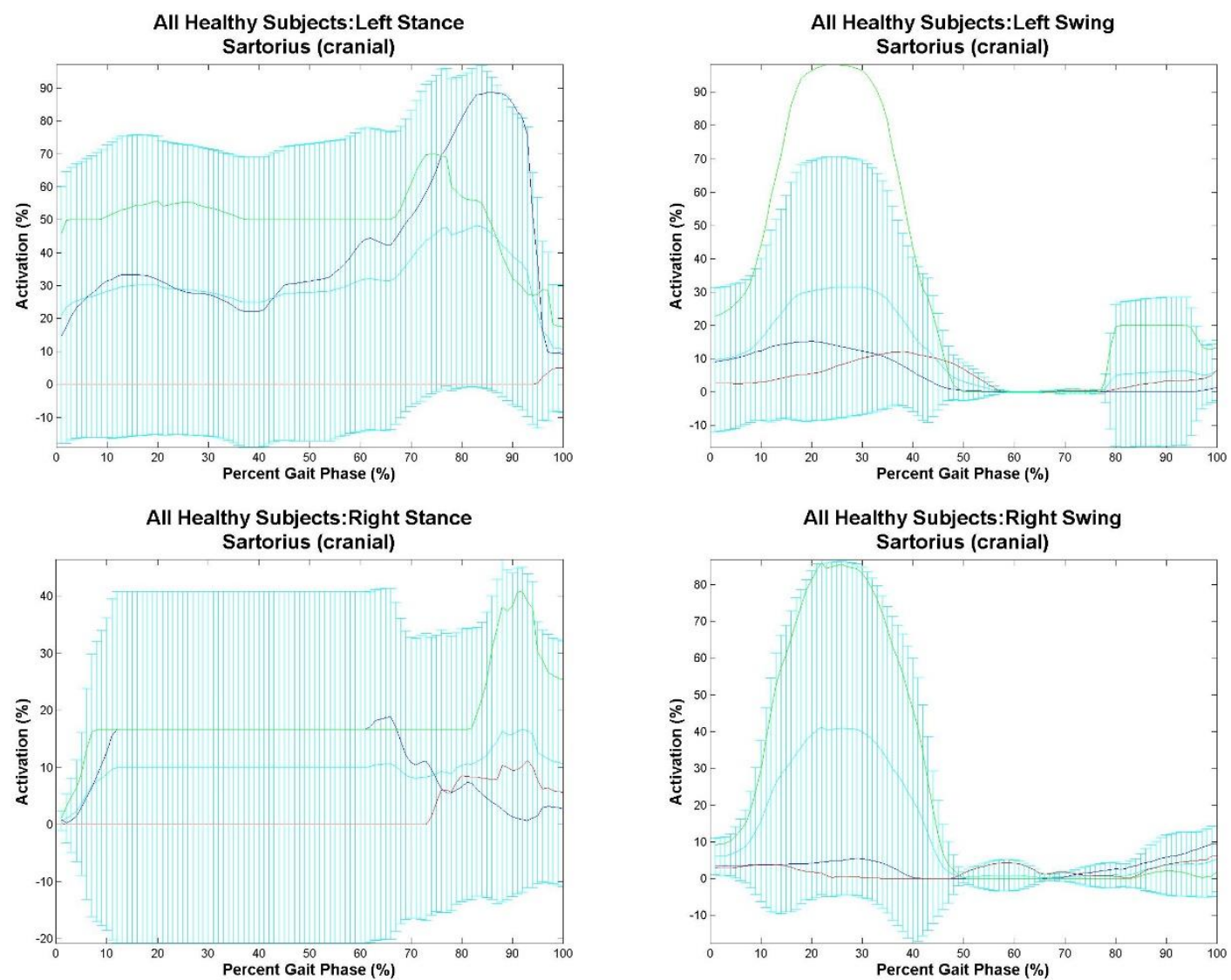

Figure $\mathrm{C} 12$. Average cranial activation of all healthy subjects. Healthy subject 1 is shown in dark blue, healthy subject 2 is shown in red, and healthy subject 3 is shown in green. Average activation of all three subjects is displayed with standard error in cyan. 

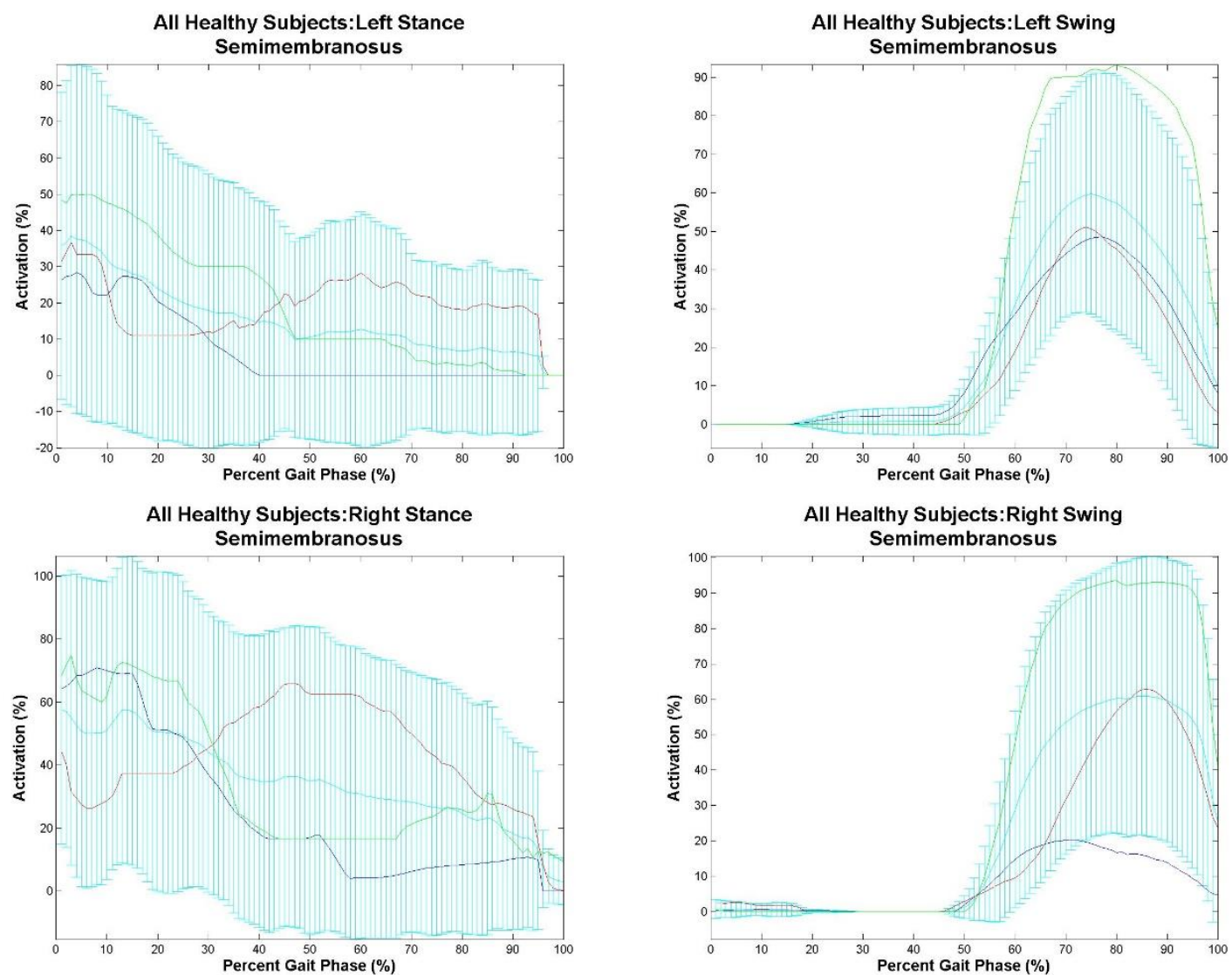

Figure C13. Average semimembranosus activation of all healthy subjects. Healthy subject 1 is shown in dark blue, healthy subject 2 is shown in red, and healthy subject 3 is shown in green. Average activation of all three subjects is displayed with standard error in cyan. 

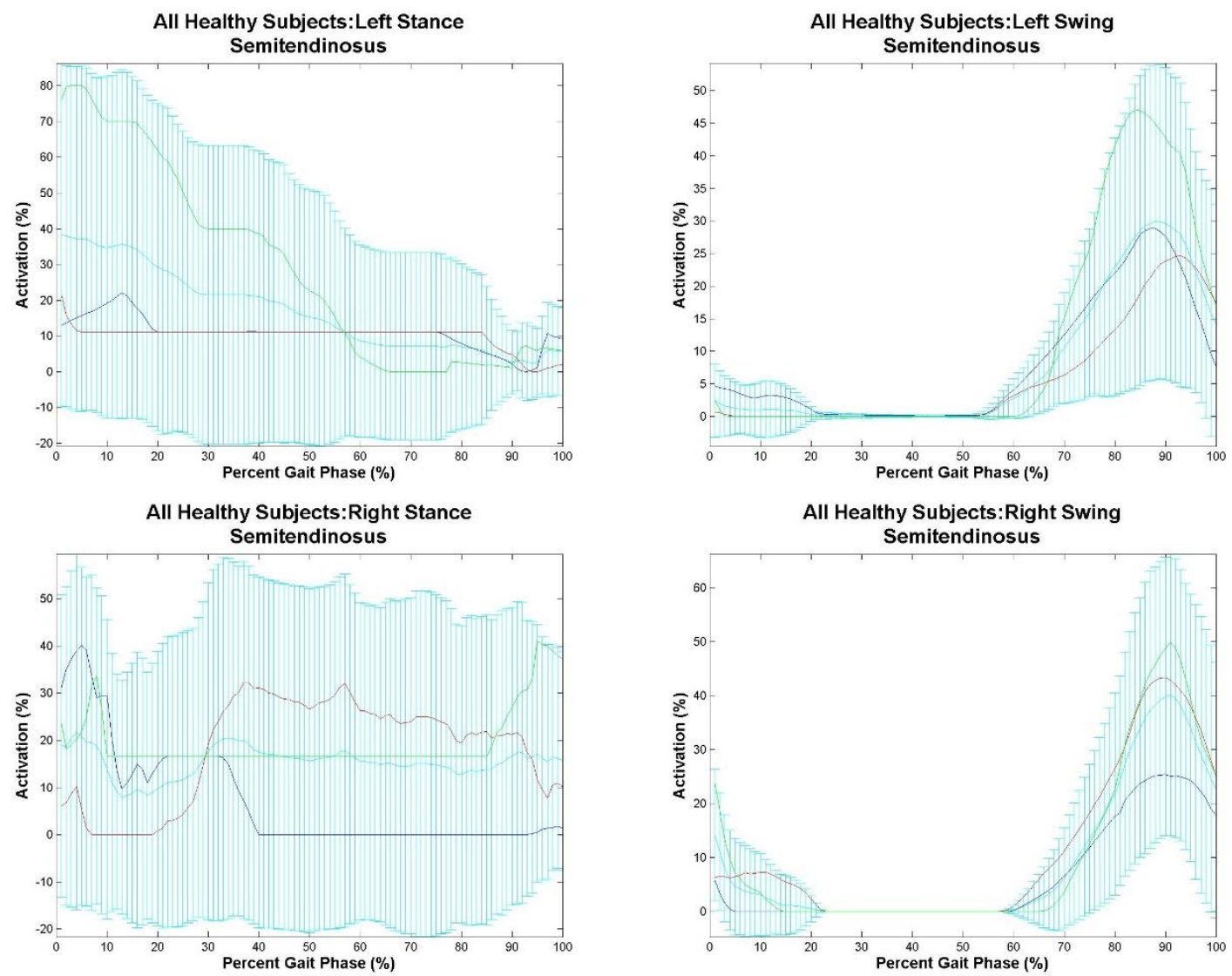

Figure C14. Average semitendinosus activation of all healthy subjects. Healthy subject 1 is shown in dark blue, healthy subject 2 is shown in red, and healthy subject 3 is shown in green. Average activation of all three subjects is displayed with standard error in cyan. 

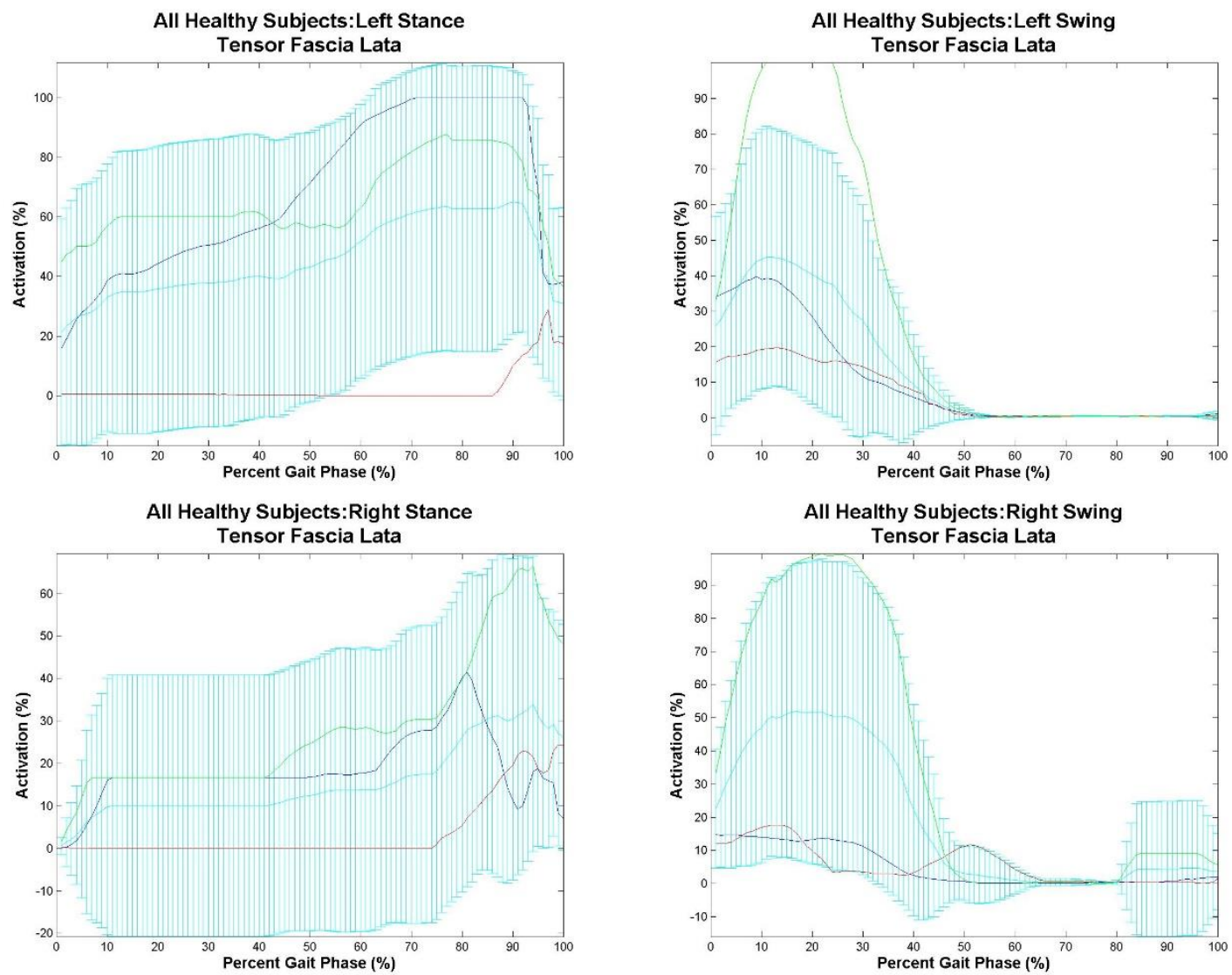

Figure $\mathrm{C} 15$. Average tensor fascia lata activation of all healthy subjects. Healthy subject 1 is shown in dark blue, healthy subject 2 is shown in red, and healthy subject 3 is shown in green. Average activation of all three subjects is displayed with standard error in cyan. 

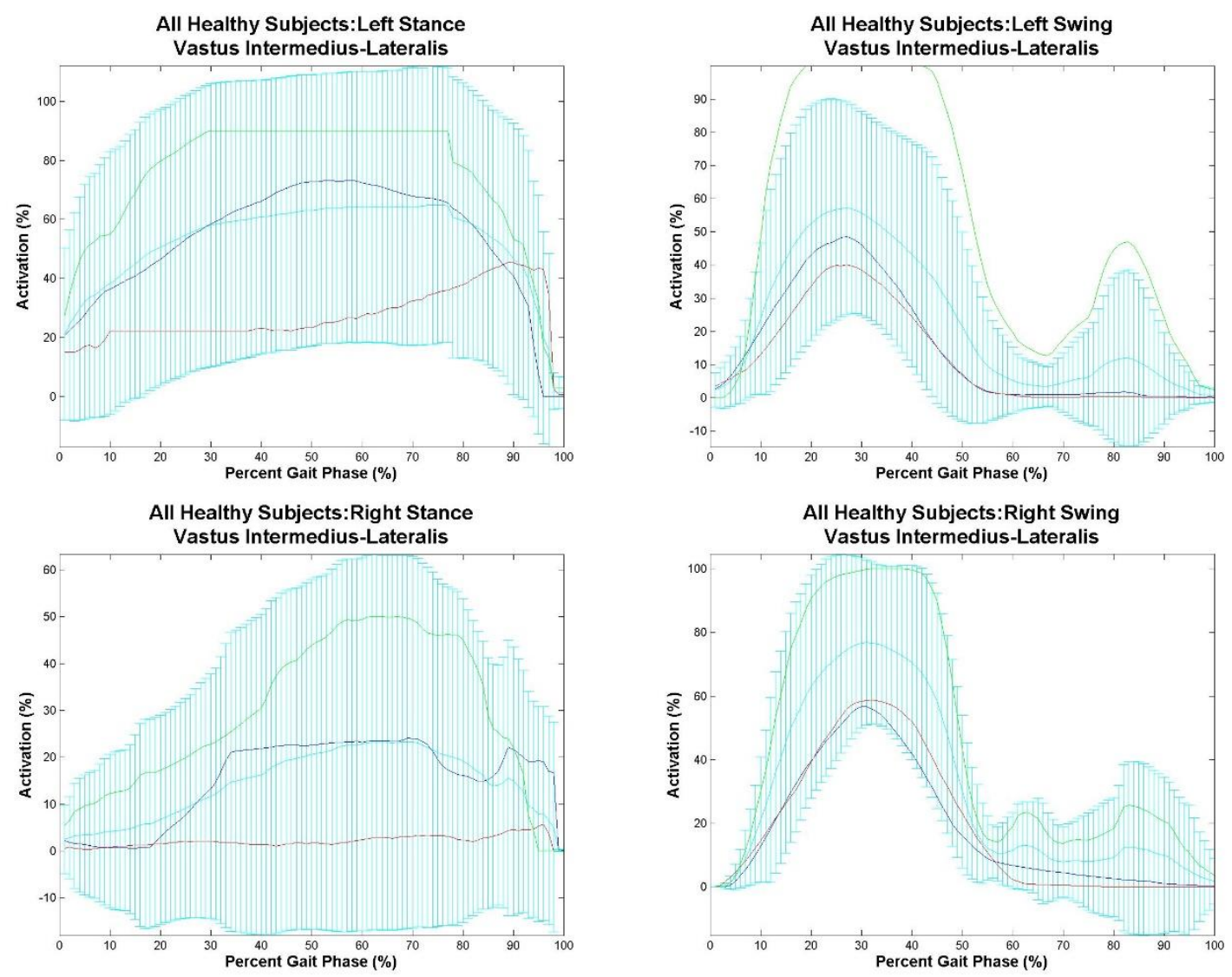

Figure C16. Average vastus intermedius/lateralis activation of all healthy subjects. Healthy subject 1 is shown in dark blue, healthy subject 2 is shown in red, and healthy subject 3 is shown in green. Average activation of all three subjects is displayed with standard error in cyan. 

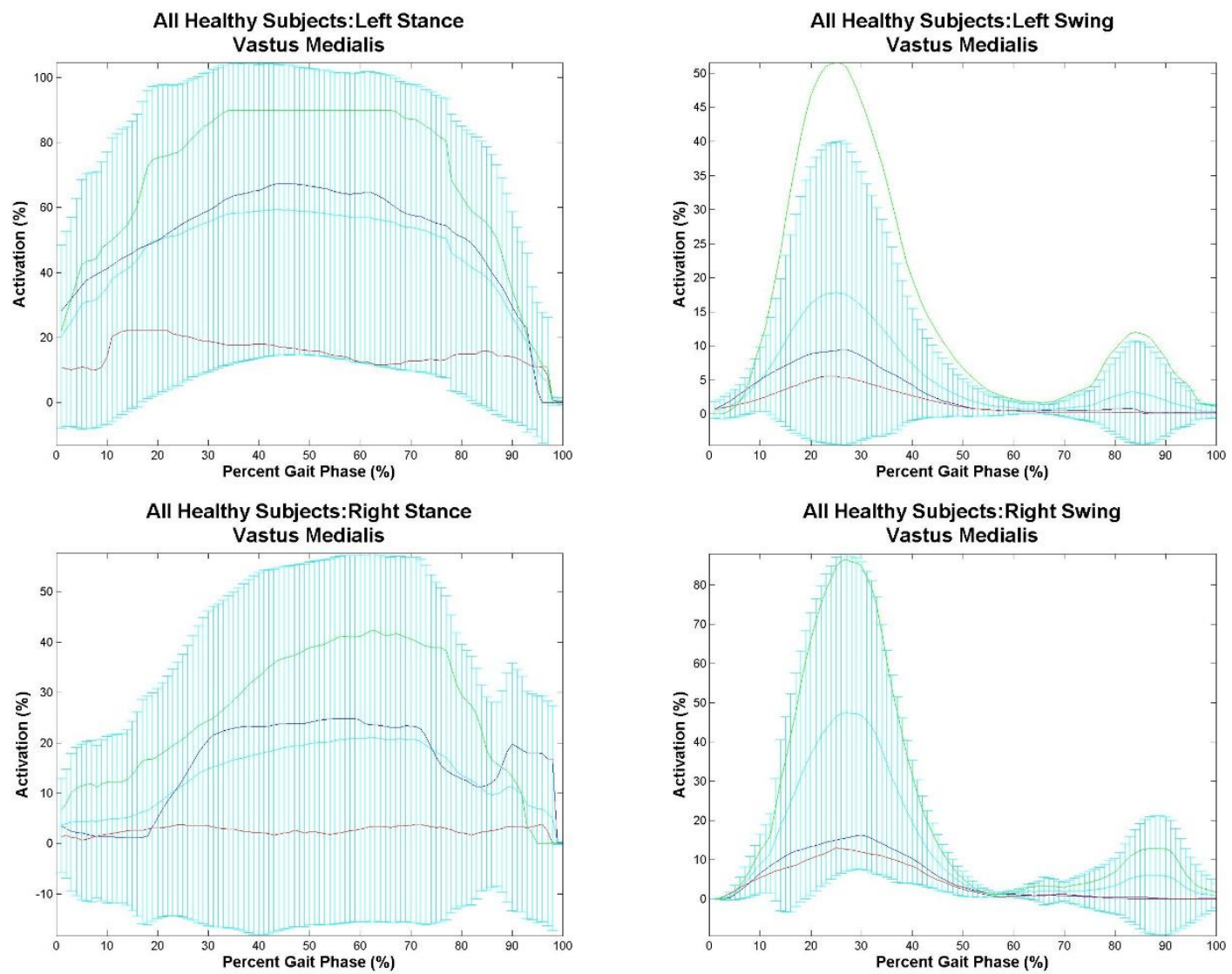

Figure C17. Average vastus medialis activation of all healthy subjects. Healthy subject 1 is shown in dark blue, healthy subject 2 is shown in red, and healthy subject 3 is shown in green. Average activation of all three subjects is displayed with standard error in cyan. 


\section{Appendix D. Body weight support system}

Developing a successful model in OpenSim requires accurate estimations of all external forces acting on the system. External forces are generally limited to ground reaction forces in healthy subjects, which can be measured using a standard force plate. However, dogs with severe IVDH are often incapable of supporting their own body weight post injury, as well as postsurgery (Levine, Levine et al. 2011). Body weight support (BWS) has been provided by holding the base of the tail (Olby, Harris et al. 2004) or using a support band situated around the abdomen (Hamilton, Franklin et al. 2007), neither of which quantify the applied force. Although these methods are adequate for rehabilitation purposes, valuable information is lost because a cost efficient means does not exist for the quantification of applied forces. Additionally, BWS information is necessary input to an OpenSim model of the early stages of injury. Therefore, a body weight support system (BWSS) was developed to wirelessly record and display the load applied during locomotion.

First, a commercially available hook scale (American Weigh Scale, Model: H110) was purchased from Amazon. Originally, the intention was to dismantle the scale and attach a custom circuit to the output to allow recording. Unfortunately, the integrated circuit was covered and held in place by an epoxy to prevent reverse engineering. Therefore, the strain gauge was removed from the circuit and connections were tested to determine function. The strain gauge was determined to be a Wheatstone bridge with two outputs. The difference between outputs was measured to be $\sim 1.1 \mathrm{mV}$ at rest; this voltage decreased with increasing weight. The output voltage was too low to accurately extrapolate applied weight. Therefore a circuit was built to amplify and filter the output. 
The circuit consists of an amplification stage centered at $0 \mathrm{~V}$ with a low pass filter. Parts for the circuit were either acquired from the bioinstrumentation lab at the University of Louisville or ordered from DigiKey. A representation of the circuit constructed using MultiSim software (National Instruments, Austin, TX) can be seen in Figure D1.

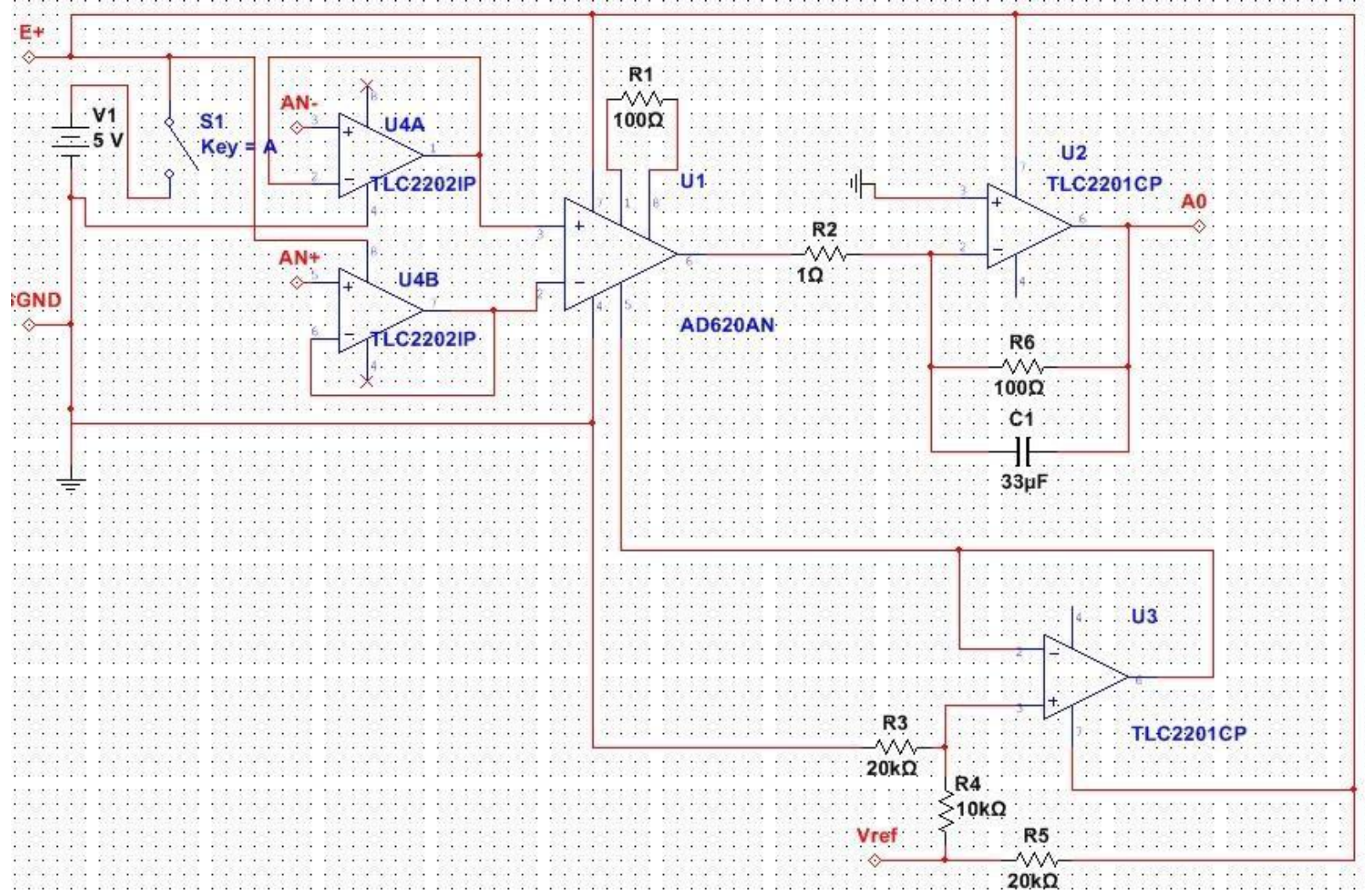

Figure D1. Schematic of the breadboard circuit represented using MultiSim. GND represents the ground wire of the strain gauge, which was attached to a common ground and to the ground pin of the Arduino board. E+, AN-, and AN+ are the other 3 leads to the strain gauge. E+ is the excitatory voltage ( $5 \mathrm{~V}$, provided by the Arduino board). $\mathrm{AN}$ - and $\mathrm{AN}+$ are the differential outputs of the strain gauge. A0 is the final readout of the circuit, which is directed into the Bluetooth module. 
The circuit was initially constructed and tested on a breadboard purchased from Radio Shack. The output (A0) was connected to the analog read pin of an Arduino Uno board. The values read in a buffer were transmitted via a Hossen serial Bluetooth module (model RS232). Values were correlated to weight using a calibration software developed using LabView software (National Instruments, Austin, TX). Output files from the calibration software were then used as input for a separate LabView program, which displayed the data in real time.

Despite initial success, the connections in the breadboard proved to be unreliable. To solve this issue, the circuit design was rebuilt and designed as a printed circuit board (Figure D2). The printed circuit board was fabricated by a third party vendor, but components were soldered in-house.

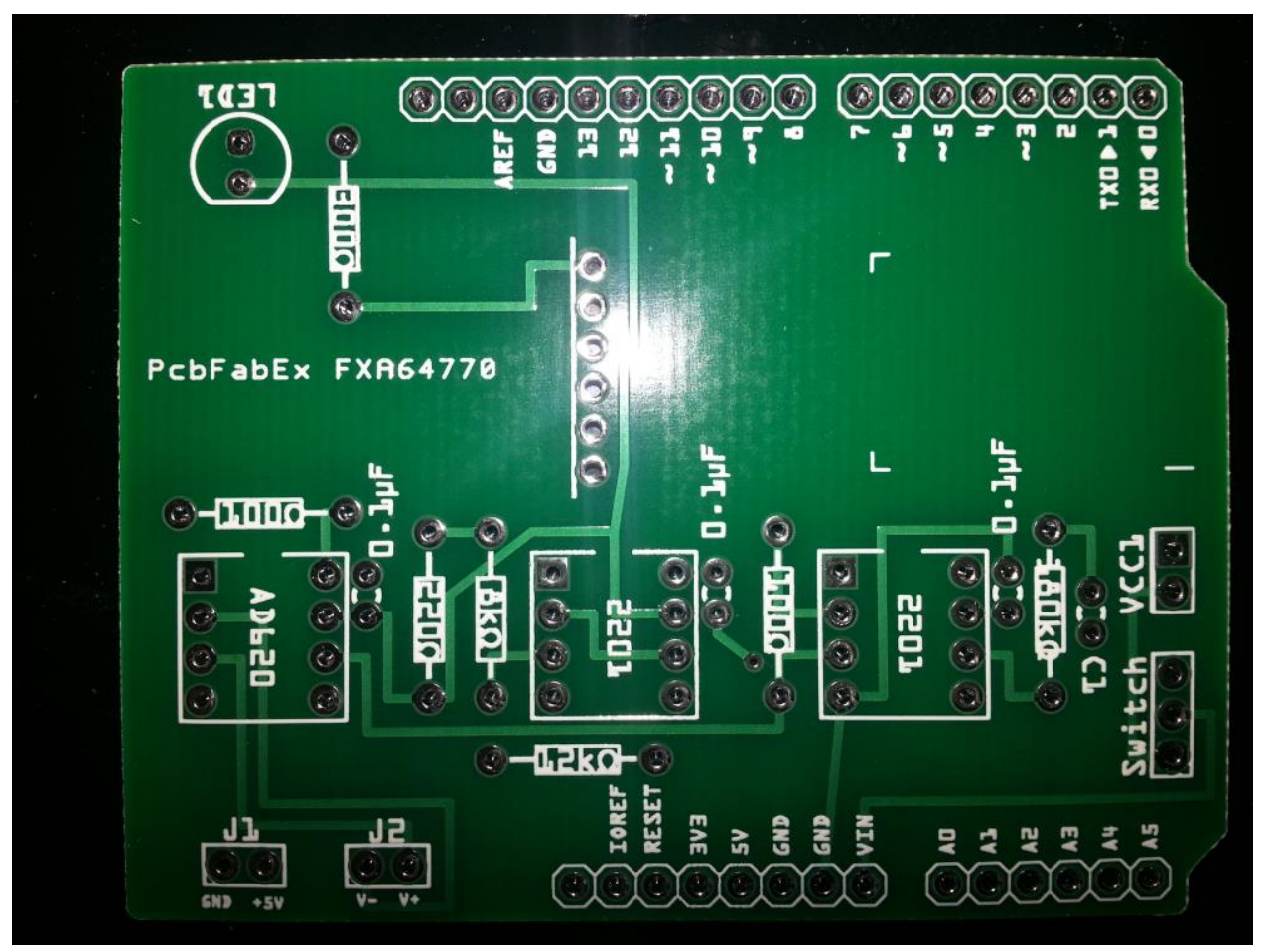

Figure D2. Final printed circuit board, without soldered components. 
The device is currently in use at the College of Veterinary Sciences at Texas A\&M University. Recommendations for future work will be briefly mentioned, although the device is capable of performing its intended functions. First, an automatic calibration system could be implemented. Thermal expansion of the materials in a strain gauge causes slight changes in resistance, which directly affects the output voltage. The scale currently requires calibration on a day to day basis, because the system does not account for temperature variations. Knowledge of the strain gauge behavior with respect to temperature could be implemented in the LabView program; this information combined with a temperature sensor could be used to eliminate the need for frequent calibration. Second, the scale resolution is limited due to the Arduino Uno board, which has a 10-bit analog to digital converter. Therefore, the resolution of the acquired force measurement is restricted to 0.18 pounds. An analog to digital convertor with higher resolution would overcome this limitation. 